ADAPTIVE ROBUST ATTITUDE CONTROLLER DESIGN FOR A QUADROTOR PLATFORM

A THESIS SUBMITTED TO

THE GRADUATE SCHOOL OF NATURAL AND APPLIED SCIENCES

$\mathrm{OF}$

MIDDLE EAST TECHNICAL UNIVERSITY

BY

EMRE YILMAZ

IN PARTIAL FULFILLMENT OF THE REQUIREMENTS

FOR

THE DEGREE OF MASTER OF SCIENCE

IN

AEROSPACE ENGINEERING

JULY 2014 

Approval of the thesis:

\section{ADAPTIVE ROBUST ATTITUDE CONTROLLER DESIGN FOR A QUADROTOR PLATFORM}

submitted by EMRE YILMAZ in partial fulfillment of the requirements for

the degree of Master of Science in Aerospace Engineering Department, Middle East Technical University by,

Prof. Dr. Canan Özgen

Dean, Graduate School of Natural and Applied Sciences

Prof. Dr. Ozan Tekinalp

Head of Department, Aerospace Engineering

Assist. Prof. Dr. Ali Türker Kutay

Supervisor, Aerospace Engineering Dept., METU

\section{Examining Committee Members:}

Prof. Dr. Yusuf Özyörük

Aerospace Engineering Department, METU

Assist. Prof. Dr. Ali Türker Kutay

Aerospace Engineering Department, METU

Dr. Volkan Nalbantoğlu

TUSAŞ Aerospace Industry, TAI

Assoc. Prof. Dr. Oğuz Uzol

Aerospace Engineering Department, METU

Assist. Prof. Dr. Ercan Gürses

Aerospace Engineering Department, METU

Date: 
I hereby declare that all information in this document has been obtained and presented in accordance with academic rules and ethical conduct. I also declare that, as required by these rules and conduct, I have fully cited and referenced all material and results that are not original to this work.

Name, Last Name: EMRE YILMAZ

Signature 


\title{
ABSTRACT
}

\section{ADAPTIVE ROBUST ATTITUDE CONTROLLER DESIGN FOR A QUADROTOR PLATFORM}

\author{
Yilmaz, Emre \\ M.S., Department of Aerospace Engineering \\ Supervisor : Assist. Prof. Dr. Ali Türker Kutay
}

July 2014, 141 pages

This thesis includes attitude controller design ideas for a quadrotor platform which can be regarded as an exceptionally agile flying robot with highly nonlinear and unstable features in flight dynamics. These platforms pose severe problems in characterizing the dynamics especially when performing high-speed manoeuvres. These facts cause the quad-rotor not to lose its popularity as a compelling tool among avid researchers who endeavour to realize various controller ideas. The procedure in this thesis is initiated with the construction of the system model and the verification of this phase relying on the characteristics of the test bed. With the aid of sensors on the off-the-shelf platform, the controllers are designed to enact tracking of the reference commands that contain the desired trajectories and attitudes. The controller methods highlighted in this research are non-linear dynamic inversion, model reference adaptive control and integral back-stepping technique. The trade-off between performance and robustness is investigated as well. The responses of the system to the impacts of 
the existence of uncertain parameters, unmatched uncertainties or disturbances are exceptional means to judge how robust the controller is. An overview of the cases with parametric uncertainty and the existence of noise, therefore, find its place as a section within this work. This sketch grades the controller options while putting forward the advantage of adaptation. The simulation results show that all controllers operate exceptionally in noiseless and noisy scenarios. Under the cases where high-level parameter uncertainties or unknown disturbances exist, however, the functionality of base controller and Integral Back-stepping can be claimed no more. In coherence with its purpose of integration into the controller, only adaptive algorithm survives these situations. The analysis including the computation of 2-norms and maximum absolute value of error vectors in the commanded state axis brings about supportive results for the deductions about the adaptation. Besides, by employing correction approaches, the advancement of the controller in terms of robustness is examined where dead zone implementation, e- and sigma-modifications are exploited. Among these modifications, e-mod and dead zone satisfy specified criteria for convergence and robustness while sigma-mod is determined as useless. In the procedure, studies about simulations with various step size values, various fixed-step ODE methods, different levels of unknown disturbances and uncertain parameters are also conducted in order to see the sensitivity of the adaptation against these criteria. The reliability of the methodologies should be justified through experiments and the analogy between experiment and theory is provided. The motivation behind this research is to produce persistent attitude controllers to lay the first stone for more complex algorithm structures such as autonomous flight phases, obstacle avoidance and way-point targeting.

Keywords: Adaptation, Robustness, Model Reference Control, Non-Linear Dynamic Inversion, Quadrotor, Attitude Controller 


\title{
ÖZ
}

\section{QUADROTOR PLATFORMLARI İÇIN UYARLANABİLİR VE GÜRBÜZ AÇISAL KONUM KONTROLCÜ TASARIMI}

\author{
Yllmaz, Emre \\ Yüksek Lisans, Havacılık ve Uzay Mühendisliği Bölümü \\ Tez Yöneticisi : Yrd. Doç. Dr. Ali Türker Kutay
}

Temmuz 2014 , 141 sayfa

Bu tez çalışması, uçuş dinamiğindeki kararlı ve doğrusal olmayan özellikleri ile üst düzey çeviklikte uçan bir robot olarak adlandırılabilecek quadrotor platformları için açısal tutum kontrolcü tasarım düşüncelerini içerir. Bu platformlarda özellikle yüksek hız manevraları sırasında dinamik özellikleri belirlemede sorunlar ortaya çıkmaktadır. Bu gerçekten yola çıkarak, quadrotorların ilgi uyandıran birer araç olarak çeşitli kontrolcü fikirlerini gerçekleştirmek için emek sarfeden hevesli araştırmacılar arasında popülaritesini kaybetmediği sonucuna varılabilir. $\mathrm{Bu}$ tezdeki süreç, temel sistem model yapısının oluşturulması ve bu fazların, test yatağının özelliklerine dayanarak doğruluğunun teyit edilmesi ile başlar. Satışa sunulmuş platformlardaki algılayıcıların yardımı ile, kontrolcüler arzu edilen yörünge ve tutumları takip etmeleri için harekete geçirilecek şekilde tasarlanır. $\mathrm{Bu}$ çalışmada bahsi geçen başlıca kontrolcü yöntemleri, doğrusal olmayan dinamik evirtim, model referans uyarlanabilir kontrol ve integral geri-adımlama teknik- 
leridir. Performans ve gürbüzlük arasındaki ödünleşim de ayrıca bu çalışmada incelenmiştir. Sistemin, belirli olmayan parametrelerin varlığına, eşleştirilememiş belirsizliklere ya da düzensizliklerin etkisine karşı verdiği tepkiler, gürbüzlügüü değerlendirilmesi için fevkalade yollardır. Bu yüzden, parametrik belirsizlik ve gürültü barındıran ölçümleri de içeren durumlara genel bir bakış, kendine bu çalışma içerisinde yer bulur. Bu şema ile, kontrolcü seçenekleri uyarlanabilirliğin faydalarını gözetecek biçimde notlandırılabilir. Bu tip kaygılarla sürdürülen benzetim çalışmalarının sonuçları bu tezde kendisine büyük bir yer bulmaktadır. Benzetimin ortaya çıkardığı ilk sonuç tüm kontrolcülerin gürültülü ve gürültüsüz durumlardaki işlevselliğidir. Ancak, yüksek seviyede parametre belirsizliği ve bilinmeyen düzensizliklerler, integral geri adım yöntemi ve temel kontrolcüden gelen sonuçların bozulması gözlenmiştir. Bu ve benzeri durumlarda ise uyarlanabilirlik işlevselliğini korumaktadır. Durum hataları için yapılan 2-norm ve azami mutlak değer analizleri sonucunda ise bu bahsi geçen konular matematiksel olarak gösterilmiş ve izah edilmiştir. Dahası, e-,sigma- ve ölü-alan uygulanması gibi çeşitli düzeltme yaklaşımlarını kullanarak, gürbüzlük açısından kontrolcülerin geliştirilmesi incelenebilir. Bu düzenlemeler arasında, e-mod ve ölü-alan yöntemleri belirlenmiş kriterlere uygun bir şekilde gürbüzlük özelliği gösterirken sigma-mod kullanımı yararsız olarak kararlaştırılmıştır. Süreç boyunca, çeşitli adım büyüklükleri, sabit adım normal diferansiyel denklem yöntemleri, bozunum ve belirsizlik seviyeleri içeren çalışmalar da uyarlanabilirliğinin etkinliğini görebilmek amacıyla yürütülmüştür. Bu yöntemlerin güvenilirliği deneylerle veya deney ile teori arasındaki uyumlarla kanıtlanmalıdır. Bu araştırma arkasındaki temel düşünce ise otonom uçuş halleri, engel önleme ve ara-nokta hedefleme gibi daha karışık yapıların ilk taşı olacak olan kararlı ve inatçı bir açısal tutum kontrolcüsü tasarlamaktır.

Anahtar Kelimeler: Uyarlanabilirlik, Gürbüzlük, Model Refrans Kontrol, Quadrotor, Doğrusal Olmayan Dinamik Evritim, Açısal Konum Kontrolcüsü 
Bu tez çalışmamı, verdikleri emek ve gösterdikleri sabır ile beni bugünlere getiren değerli annem ve babam ile her zaman benim için gurur kaynă̆ı olmuş kardeşime ithaf etmek istiyorum. 


\section{ACKNOWLEDGEMENTS}

First and foremost, my deepest gratitude is to my supervisor, Ali Türker Kutay. It was a great honour to work with him for the last three years and our cooperation influenced my academical view highly. When I requested his help during the difficult and confusing times both in the duration of my thesis study and in my Ph.D. applications, he never hesitated to provide reference for me, share his opinion and give feedback. I really appreciate his constant support, motivation and guidance. Life leads many to quite surprising paths. It is a great joy for me to have the chance to follow his steps.

I am indebted to Ozan Gözcü, Harun Özkanaktı, Pelin Ergen and Alpay Demircan for their brotherhood, invaluable support and the wonderful memories and moments shared. Anatolian-Craft is not only a team but also a family for us now. Besides, Harun performed an amazing job while designing and manufacturing the arm mechanisms and I am terribly grateful to his efforts.

My deepest thanks are also for Özgür Harputlu and Özgür Tümüklü. From the beginning of my graduate research assistantship, without their support, motivation, companion and cheering, nothing would be that easy in this department for me. Besides, I would like to thank Özge Sinem Özçakmak, Özgür Yalçın, Özcan Yırtıcı, Can Çıtak, Tuğçe Garip, Ayşe Hazal Altuğ, Pınar Eneren, Engin Leblebici, Muharrem Özgün, and Kenan Cengiz, the best colleagues one can have in a department. Their fellowship and fun turned the dullness of our floor into an epic place one can never leave (literally!). Here, I have to mention two people from Çöker Group who make the department a more bearable place for me. Especially, the concept of coffee breaks and talks had never been more soul-replenishing. Imren Uyar and Miray Arca, thank you girls.

I appreciate the cooperation, teamwork and friendship of Emrecan Suiçmez and Derya Kaya in the quadrocopter project. The helps of my friend, Anas Ab- 
durrahim, about the wind tunnel and the efforts of Emre Mutlu for the circuit model were invaluable. I would like to thank Senem Ayşe Haser, Yunus Tansu Aksoy, Abdulkerim Çengeloğlu, Volkan Çoşgun, Volkan Yıldırım, Levent Cevher, Başar Kütükoğlu, Denizhan Yavaş, Murat Şenipek, Burak Sarsılmaz, and Başak Zeka for their enjoyable company in the department. Lately, the department has been updated with the best Structure Lab ever although we had a short time together. My best wishes and thanks are also for the crew, İlhan Ozan Tunçöz, Harun Tıraş, Yosheph Yang, Pınar Arslan, Uğur Kalkan and Caner Durak. There are a lot of people that were with me in my M.S. years. My dearest friends, Şemsettin Balta, Züte, Çağıl Köseoğlu, Yiğit Atılgan, Tuanna Demir, Mustafacan Karadayı, Serkan Kaymak, Sezer Gül, and my beloved family, Selanur-Selnur Canaz, Serap Yürük, İrem-Verda-Dilek-Hüseyin-Hasbiye Yllmaz and Sedat-Sabriye-Nuran Pinçe, I am so lucky to have you by my side.

I shall thank my esteemed professors, Demirkan Çöker, Yusuf Özyörük, Ercan Gürses, İlkay Yavrucuk and Oğuz Uzol for taking their time for never-ending reference letters, beneficial talks and the motivation they provided during my application period. I would also like to thank to the members of my thesis advisory and exam committee, Yusuf Özyörük, Volkan Nalbantoğlu, Oğuz Uzol and Ercan Gürses for their acceptance of my invitation. My special thanks go to Betil Başeğmezler for the chance to be a part of her class for so many years. My graduate research is supported by TÜBİTAK-BİDEB Graduate Scholarship (2211) and RÜZGEM. The author gratefully acknowledges these supports.

The mentorship of my cousin, Çerağ Pinçe, eased the situation for me while taking probably one of the most difficult decisions I have made for all these years. I am incredibly indebted to his support at the moment of despair. It is a fortune to have such a nice man and wise academician as a relative.

Kaan Bilgehan and Gökhan Arıkan, I am thankful to both of you for many things. Hard times indeed revealed my true friends. I cannot think of any proper words that could describe your unbounded support and there are also none that could convey my gratitude to you. Finally, I would like to thank my father, my mother and my brother again for being so supportive and understanding. 


\section{TABLE OF CONTENTS}

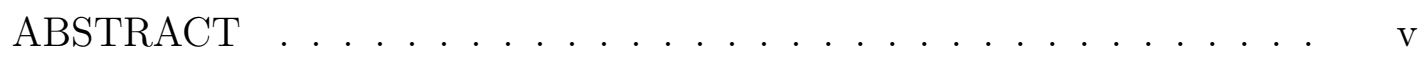

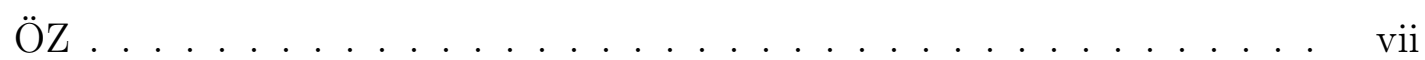

ACKNOWLEDGEMENTS . . . . . . . . . . . . . . .

TABLE OF CONTENTS . . . . . . . . . . . . . . . . xii

LIST OF TABLES . . . . . . . . . . . . . . . . . . . . . . . . . xvi

LIST OF FIGURES . . . . . . . . . . . . . . . . . . . . . . . . . . xvii

LIST OF ABBREVIATIONS . . . . . . . . . . . . . . . . . . . xxi

LIST OF SYMBOLS . . . . . . . . . . . . . . . . . . . . . . . xxii

CHAPTERS

$1 \quad$ INTRODUCTION $\ldots \ldots \ldots \ldots \ldots$

1.1 Autonomy, Robotics and Uninhabited Aerial Vehicles. . 1

1.1 .1 Defining Autonomy . . . . . . . . . . . . 1

1.1.2 Robotics and Links to Autonomy. . . . . . . . 2

1.1.3 History of Autonomy and Robots . . . . . . . 3

1.1.4 Works of Previous Century for the Development of Autonomy and Robotics . . . . . . . . . . . 4

1.1.5 Current Field of Use of Robots and Autonomy in Robotics . . . . . . . . . . . 6

1.1.6 Levels of Autonomy in UAV Technology . . . . 11

1.1.7 Unmanned Rotary Aerial Vehicle Systems . . . 14

1.2 The Concept of Quadrocopters . . . . . . . . . . . 15

$1.2 .1 \quad$ History $\ldots \ldots \ldots \ldots \ldots \ldots$

$1.2 .2 \quad$ Reason of Interest for Quadrocopters . . . . . 16

1.2.3 State-of-Art and Literature Survey for Quadrotors 19 
$1.3 \quad$ Motivation, Objectives and Content of This Thesis . . . 28

2 MODELING. . . . . . . . . . . . . . . . . . . . . . . . . . . 31

$2.1 \quad$ System Dynamics $\ldots \ldots \ldots$. . . . . . . . . . . . . 31

$2.2 \quad$ Geometry and Propeller Dynamics . . . . . . . . . . . 32

2.2.1 The Influence of Propeller Force on Body Center of Mass . . . . . . . . . . . . . . . . . . 32

2.2.2 The Influence of Propeller Torque on Body Center of Mass . . . . . . . . . . . . . . . . 34

2.2.3 Total Moment Exerted on Body Center of Mass 35

$2.3 \quad$ Rigid Body Dynamics . . . . . . . . . . . . . . . . 35

$2.3 .1 \quad$ Rotation Matrix . . . . . . . . . . . . 35

$2.3 .2 \quad$ Rate of Attitude Propagation Equations . . . . 37

2.3.3 Angular Momentum Dynamics . . . . . . . . . 38

$2.4 \quad$ Other Considerations for Modelling the Dynamics . . . 39

2.5 State-Space Representation of Attitude Dynamics . . . . 40

3 CONTROL STRATEGIES . . . . . . . . . . . . . . . . . 43

$3.1 \quad$ Non-Linear Dynamic Inversion . . . . . . . . . . . . . 43

$3.1 .1 \quad$ Regarded Dynamic System . . . . . . . . . . 43

$3.1 .2 \quad$ Dynamic Inversion . . . . . . . . . . . . . . 44

3.1.3 The Concept of Relative Degree . . . . . . . . 45

3.1.4 The Relative Degree of Multi-Variable Systems 45

3.1.5 The Transformation of the System Dynamics . 48

$3.1 .6 \quad$ Linearization of State Feedback. . . . . . . . . 49

3.1.7 Non-Linear Dynamic Inversion of Attitude Dynamics of a Quadrocopter . . . . . . . . . 50

$3.2 \quad$ Reference Model and Error Dynamics . . . . . . . . . 51

3.2.0.1 The Thrust Model of the Motor and Propeller Dynamics . . . . . . . . 55

$3.2 .1 \quad$ Parameter Selection . . . . . . . . . . . 55

$3.3 \quad$ Pseudo-Control Hedging $(\mathrm{PCH}) \ldots \ldots$. . . . . . . 58

$3.4 \quad$ Model Reference Adaptive Control . . . . . . . . . . . . 63

3.4.1 Adaptive Rate Controller within MRAC Structure 65 
$3.4 .2 \quad$ Lyapunov's Direct Method . . . . . . . . . . 69

3.4.3 Lyapunov Analysis for Adaptive Rate Controller 70

3.4.4 Attitude Loop within MRAC Structure . . . . 74

$3.5 \quad$ Robustness Modifications to MRAC . . . . . . . . 76

3.5.1 Lyapunov Analysis for Unmatched Uncertainties 76

3.5.2 Dead-Zone Modification . . . . . . . . . 78

3.5.3 Sigma-Modification . . . . . . . . . . 78

3.5.4 e-Modification . . . . . . . . . . . . . 79

$3.6 \quad$ Integral Back-stepping . . . . . . . . . . . . . . . . . 80

$4 \quad$ RESULTS AND DISCUSSION FOR VARIOUS CONTROLLER

OPTIONS ....................... 83

$4.1 \quad$ Simulation Notes . . . . . . . . . . . . . . . . . . . . . . 83

$4.2 \quad$ Simulation Results for Constant Attitude Tracking . . . 85

$4.3 \quad$ Simulations Results for Sinusoidal Input with Various Periods. . . . . . . . . . . . . . . 90

$4.4 \quad$ Error Norms of the Simulation Results . . . . . . . . . . 92

$4.5 \quad$ Discussion for Simultaneous Inputs in 2-Different Axes . 94

$4.6 \quad$ Simulation Results with Various Step-size Values . . . . 95

$4.7 \quad$ Simulations Results with Various Fixed-Step ODE Solver Methods . . . . . . . . . . . . . 99 97

$4.8 \quad$ Simulations Results with Various Levels of Disturbances 98

$4.9 \quad$ Simulations Results with Various Levels of Uncertain Parameters .................. 100

5 RESULTS AND DISCUSSION FOR ADAPTATION AND ROBUSTNESS MODIFICATIONS . . . . . . . . . . . . . . 103

$5.1 \quad$ Adaptation Gains for Simulations . . . . . . . . . . . . 103

$5.2 \quad$ Simulation Results and Discussion for Robustness Modifications and Unmodified Adaptation . . . . . . . . . 107

6 C CONCLUSION . . . . . . . . . . . . . . . . . . 111

REFERENCES . . . . . . . . . . . . . . . . . . . . . . . . . . . . 113

A $\quad$ FURTHER CONSTANT TRAJECTORY RESULTS . . . . . . 127

B $\quad$ FURTHER SINUSOIDAL INPUT RESULTS . . . . . . . . . . 133 
C FURTHER MODIFICATION RESULTS . . . . . . . . . . . . . 139

D FURTHER MODIFICATIONH RESULTS . . . . . . . . . . . . 141 


\section{LIST OF TABLES}

\section{TABLES}

Table 1.1 UAV examples $\ldots \ldots \ldots$. . . . . . . . . . . . . . . . . . . . . . . . . . . . 11

Table 1.2 The Description of Autonomy Levels . . . . . . . . . . . . . . 13

Table 4.1 Parameters . . . . . . . . . . . . . . . . . . . . . . . . . . . . 83

Table 4.2 Input Types . . . . . . . . . . . . . . . . . . . . . 84

Table 4.3 Simulation Scenarios with their figure numbers . . . . . . . . 85

Table 4.4 Figures regarding Simulations with Sinusoidal Inputs under Parameter Uncertainty organized according to their Amplitude and Periods . . . . . . . . . . . . . . . . . . . 90

Table 4.5 2-Norm of the Error Vectors of the Control Methods for Different Scenarios and Inputs . . . . . . . . . . . . . . . . . . . . 93

Table 4.6 Maximum Absolute Values of the Error Vectors of the Control Methods for Different Scenarios and Inputs . . . . . . . . . . . . . . 94

Table 4.7 Various Step Sizes Used for Simulations . . . . . . . . . . . . 95

Table 4.8 Comparison of Fixed-Step ODE Solver Types for Simulation . 97

Table 4.9 Disturbance Levels for the Case with Various Unknown Distur-

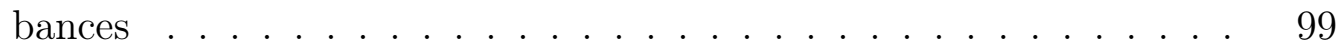

Table 4.10 Uncertainty Levels for the Case with Various Uncertain Param-

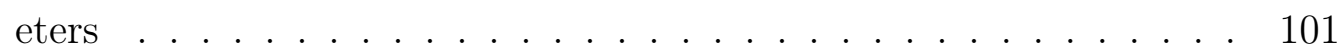

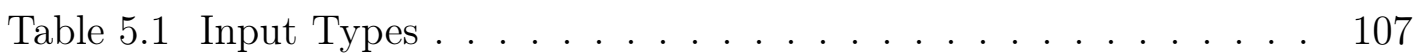

Table 5.2 Simulation Scenarios with their figure numbers . . . . . . . . 107

Table 5.3 Modification Types in Figures . . . . . . . . . . . . . . . . . . 107 


\section{LIST OF FIGURES}

\section{FIGURES}

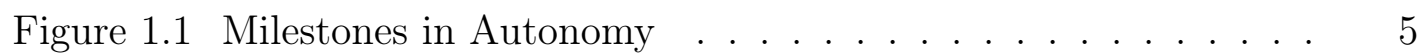

$\begin{array}{llll}\text { Figure } & 1.2 & \text { Robotic and Autonomy Examples from Current Fields of Use } & 7\end{array}$

Figure 1.3 UAVs and their Fields of Use . . . . . . . . . . . . . . . . . . 10

Figure 1.4 The Perspectives suggested in DoD Report for Autonomous

Systems . . . . . . . . . . . . . . . . . . . . . 14

Figure 1.5 First generation of rotorcrafts and quadrotors . . . . . . . . . 16

Figure 1.6 Motion Primitives for a Quadrotor . . . . . . . . . . . . . . 18

Figure 1.7 Research on Quadrocopters . . . . . . . . . . . . . . . . . . . 28

Figure 2.1 The Axis \& Movement Definitions for a Quadrotor . . . . . 32

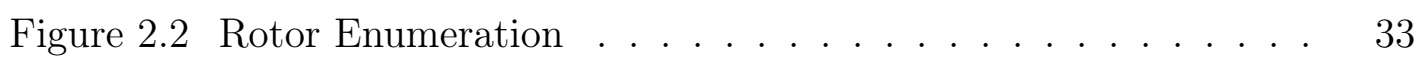

Figure 2.3 Modelled Dynamics Blocks for Quadrotor . . . . . . . . . . . 41

Figure 3.1 Scheme of State Feedback . . . . . . . . . . . . . . . . . . . 52

Figure 3.2 The Thrust Dynamics Model . . . . . . . . . . . . . . . . . 55

Figure 3.3 Structure of the Reference Model $\ldots . . . . . . . .57$

Figure 3.4 Responses Model and Error Controller State Responses for

Various Natural Frequency Values . . . . . . . . . . . . . . . . . 59

Figure 3.5 Error Controller State Responses for Various Integral Gains . 60

Figure 3.6 Reference Model Structure with and without Pseudo-Control Hedging . . . . . . . . . . . . . . . . . . 62

Figure 3.7 The General Structure of MRC . . . . . . . . . . . . . . . . 63

Figure 3.8 The General Structure of MRAC . . . . . . . . . . . . . . . 64

Figure 3.9 Modified Cascaded Controller Structure for Adaptive Laws . 65

Figure 3.10 Model Reference Adaptive Controller Structure with Adaptive Gains . . . . . . . . . . . . . . . . 67 
Figure 3.11 Reference Model Structure with Pseudo-Control Hedging . . 76

Figure 3.12 Gain Adjustments for Integral Backstepping Method . . . . . 81

Figure 4.1 Simulation of fixed angular orientation control with no noise 86

Figure 4.2 Simulation of fixed angular orientation control with noise addition . . . . . . . . . . . . . . . . . . . 87

Figure 4.3 Simulation of fixed angular orientation control with a different quadrotor model . . . . . . . . . . . . . . . . . . . . 88

Figure 4.4 Simulation of fixed angular orientation control in the presence of unknown disturbance . . . . . . . . . . . . . . . . . . . . . . . . . 89

Figure 4.5 Simulation of sinusoidal command with various periods and amplitudes in the presence of uncertain parameter . . . . . . . . . . 91

Figure 4.6 The Simulation of 2-Axis Fixed Orientation Commands in the Presence of Uncertain Parameters and Unknown Disturbances in Roll with Various Step-sizes . . . . . . . . . . . . . . . . . . . . . . . 96

Figure 4.7 Simulation results of the constant orientation tracking in the existence of parameter uncertainty via various Runge-Kutta methods 98

Figure 4.8 Simulation results of fixed angular orientation in the presence of various levels of unknown disturbances . . . . . . . . . . . . . . . 99

Figure 4.9 Simulation results of fixed angular orientation in the presence of various uncertain parameters . . . . . . . . . . . . . . 101

Figure 5.1 MRAC Adaptation Gains related to State Outputs in the simulation of attitude control for roll step-input tracking . . . . . . . . 104

Figure 5.2 MRAC Adaptation Gains related to State Outputs in the simulation of attitude control for roll step-input tracking (continued) . . 105

Figure 5.3 MRAC + Dead-Zone Modification Adaptation Gains related to State Outputs in the simulation of attitude control for roll step-input tracking . . . . . . . . . . . . . . . . 106

Figure 5.4 State Error Responses of Various MRAC Robustness Modifications for Step Roll-Input Trajectory Tracking in the presence of noise and unknown disturbance . . . . . . . . . . . . . . . . . . 109 
Figure 5.5 State Error Responses of Various MRAC Robustness Modifications for Step Roll and Pitch-Input Trajectory Tracking in the presence of noise, parameter uncertainty and unknown disturbance . 110

Figure A.1 Simulation of angular control for fixed angular orientation tracking in two axes with no noise . . . . . . . . . . . . . . . 127

Figure A.2 Simulation of angular control for sinusoidal tracking in two axes with noise addition. . . . . . . . . . . . . . . . . . . . . 128

Figure A.3 Simulation of angular control for sinusoidal tracking in two axes with a different quadrotor model . . . . . . . . . . . . . . . . 129

Figure A.4 Simulation of angular control for sinusoidal tracking in two axes in the presence of unknown disturbance . . . . . . . . . . . . . 130

Figure A.5 Simulation of angular control for fixed angular orientation tracking in two axes with no noise (cont.) . . . . . . . . . . . . . . . 131

Figure B.1 Simulation of angular control for sinusoidal tracking with no

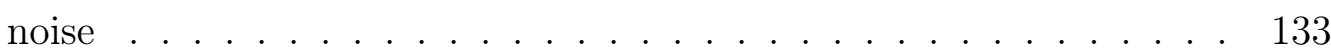

Figure B.2 Simulation of angular control for sinusoidal tracking with noise addition . . . . . . . . . . . . . . . . . 134

Figure B.3 Simulation of angular control for sinusoidal tracking with a different quadrotor model . . . . . . . . . . . . . . . . . . . . . 135

Figure B.4 Simulation of angular control for sinusoidal tracking in the presence of unknown disturbance . . . . . . . . . . . . . . . . . 136

Figure B.5 Simulation of sinusoidal command with various periods and amplitudes in the presence of unknown disturbance . . . . . . . . . 137

Figure B.6 Simulation of sinusoidal command with various periods and amplitudes in the presence of unknown disturbance . . . . . . . . . 138

Figure C.1 State Error Responses of Various MRAC Robustness Modifications for Step Roll-Input Trajectory Tracking in the presence of noise, parameter uncertainty and unknown disturbance . . . . . . . 139

Figure C.2 State Error Responses of Various MRAC Robustness Modifications for Step Roll and Pitch-Input Trajectory Tracking in the presence of noise and unknown disturbance . . . . . . . . . . . . . . 140 
Figure D.1 State Error Responses of Various MRAC Robustness Modifications for Step Roll-Input Trajectory Tracking in the presence of noise, parameter uncertainty and unknown disturbance . . . . . . . 141 


\section{LIST OF ABBREVIATIONS}

$\begin{array}{ll}\text { AFCS } & \text { Automatic Flight Control Systems } \\ \text { ALFUS } & \text { Autonomy Levels for Uninhabited Systems } \\ \text { ALFURS } & \text { Autonomy Levels for Uninhabited Rotorcraft Systems } \\ \text { AUV } & \text { Autonomous Unmanned Vehicle } \\ \text { BS } & \text { Bogacki Shampine } \\ \text { DoD } & \text { Department of Defence } \\ \text { DOF } & \text { Degree of Freedom } \\ \text { DP } & \text { Dormand-Prince } \\ \text { EOD } & \text { Explosive Ordnance Disposal } \\ \text { HALE } & \text { High Altitude Long Endurance } \\ \text { IMU } & \text { Inertial Measurement Unit } \\ \text { LQ } & \text { Linear Quadratic } \\ \text { LQR } & \text { Linear Quadratic Regulator } \\ \text { MAV } & \text { Micro Aerial Vehicle } \\ \text { MPC } & \text { Model Predictive Control } \\ \text { MRAC } & \text { Model Reference Adaptive Control } \\ \text { ODE } & \text { Ordinary Differential Equation } \\ \text { OODA } & \text { Observe Orient Decide Act } \\ \text { PI } & \text { Proportional Integral } \\ \text { PID } & \text { Proportional Integral Derivative } \\ \text { RC } & \text { Remotely Controlled } \\ \text { RK } & \text { Runge Kutta } \\ \text { ROV } & \text { Remotely Operated Vehicle } \\ \text { RUAV } & \text { Rotary Uninhabited Aerial Vehicle } \\ \text { SUAV } & \text { Small Uninhabited Aerial Vehicle } \\ \text { UAS } & \text { Uninhabited Aerial System } \\ \text { UAV } & \text { Uninhabited Aerial Vehicle } \\ \text { UCAV } & \text { Uninhabited Combat Aerial Vehicle } \\ \text { UGV } & \text { Uninhabited Ground Vehicle } \\ \text { VTOL } & \text { Vertical Take-off \& Landing } \\ & \end{array}$




\section{LIST OF SYMBOLS}

\begin{tabular}{|c|c|}
\hline$\phi$ & Roll Angle \\
\hline$\theta$ & Pitch Angle \\
\hline$\psi$ & Yaw Angle \\
\hline$x_{b}, y_{b}, z_{b}$ & Body-fixed Frame \\
\hline$x_{E}, y_{E}, z_{E}$ & Earth-fixed Frame \\
\hline$l_{\text {arm }}$ & arm length from the rotor center to the body center \\
\hline$F_{i}$ & Force vectors according to rotor numbers, i \\
\hline$r_{i}$ & $\begin{array}{l}\text { Vectors from the rotor center to the body center according to } \\
\text { rotor numbers, i }\end{array}$ \\
\hline$n_{i}$ & RPMs according to rotor numbers, i \\
\hline$K_{T}$ & Thrust Coefficient of a Propeller \\
\hline$T_{P}$ & Propeller Thrust \\
\hline$K_{Q}$ & Torque Coefficient of a Propeller \\
\hline$Q_{P}$ & Propeller Torque \\
\hline$D$ & Propeller Diameter \\
\hline$\rho$ & density \\
\hline$k_{m}$ & The Linear Relationship between Propeller Torque and Force \\
\hline$M_{\text {prop }}$ & Moment generated by propeller blades in their axis of rotation \\
\hline$p, q, r$ & Body rates \\
\hline$\dot{\phi}, \dot{\theta}, \dot{\psi}$ & Euler rates \\
\hline$M_{x}, M_{y}, M_{z}$ & Body-frame moments \\
\hline$L, M, N$ & Body-frame moments \\
\hline$T_{c}$ & Total Thrust produced by Quadrotor in vertical axis \\
\hline$\vec{H}$ & Angular Momentum vector \\
\hline$\delta m$ & mass element \\
\hline$I_{x x}, I_{y y}, I_{z z}$ & Moments of Inertia \\
\hline$I_{x y}, I_{x z}, I_{y z}$ & Products of Inertia \\
\hline$\omega$ & Angular velocity of quadrotor \\
\hline$\dot{\omega}$ & Angular acceleration of quadrotor \\
\hline$\omega_{B}$ & Angular velocity of quadrotor w.r.t body frame \\
\hline$\dot{\omega}_{B}$ & Angular acceleration of quadrotor w.r.t body frame \\
\hline$v$ & Pseudo control variables \\
\hline$\nu$ & Alternative control variables \\
\hline$u$ & control input \\
\hline$D_{x}$ & Range of system states \\
\hline & Range of system control inputs \\
\hline
\end{tabular}




$\begin{array}{ll}L_{i} & \text { Lie derivative w.r.t. the indicated function i } \\ r_{i} & \text { Relative degree w.r.t. the indicated state i } \\ \omega_{0} & \text { natural frequency of the reference model } \\ \zeta & \text { damping ratio of the reference model } \\ \phi_{r e f}, \theta_{r e f}, \psi_{r e f} & \text { body rates of reference model } \\ \phi_{c m d}, \theta_{c m d}, \phi_{c m d} & \text { body rates commands to reference model } \\ \delta_{i} & \text { the difference between the second derivative of the output and } \\ & \text { the pseudo control for state i } \\ e_{v} & \text { vector of error states } \\ k_{p}, k_{i}, k_{d} & \text { Controller gains } \\ F_{p h a s e} & \text { Force generated from the propellers for a specific flight phase } \\ \Theta_{c} & \text { Controller Parameter Vector } \\ \hat{v} & \text { Estimation form of pseudo control variables } \\ d & \text { constant disturbance vector } \\ A_{p} & \text { linear part of the system matrix } \\ B_{p} & \text { control matrix } \\ x_{p} & \text { system states } \\ f\left(x_{p}\right) & \text { the function designating the non-linearities in the system } \\ \Theta_{i} & \text { Adaptive gain matrices for state i } \\ \Theta_{i} & \text { Ideal gain matrices for state i (asterisk) } \\ T_{p, q, r} & \text { Periods regarding the body rates } \\ A_{M}, B_{M} & \text { Matrices regarding the Reference Model } \\ \sigma & \text { Variables for } \sigma \text { modification } \\ A_{p u l s e} & \text { Amplitude of the pulse wave } \\ w_{p u l s e} & \text { Width of the pulse wave } \\ T_{p u l s e} & \text { Period of the pulse wave } \\ P_{p u l s e} & \end{array}$




\section{CHAPTER 1}

\section{INTRODUCTION}

\subsection{Autonomy, Robotics and Uninhabited Aerial Vehicles}

\subsubsection{Defining Autonomy}

The readers with the will to comprehend the principal motivation of this study should steer their thoughts to concentrate on the concept of autonomy. In order to constitute an interpretation of the background of this concept, it is essential to investigate the etymological background of the word "autonomy". Derived from the language of Ancient Greeks, "autonomos", the exact equivalence of autonomy, is composed of two separate words which are "auto" and "nomos", respectively standing for "self" and "law" in modern English. Therefore, a sufficiently meaningful deduction from the combination of these words may be "the one who determines the rules for oneself" or briefly "the one who is self-governing". On a broader perspective, it is safe to assert that all these vocabulary including autonomy, autonomous, automata, automation and automatic originated from the same ancestor: "automaton". First recorded use of automaton by Homer in 749th line of the fifth book in Iliad to describe "the gates of heaven to automatically thunder open" does not take it away from autonomos as the meaning still points out the"acting of one's own will" [1]. Coming back to today's jargon, automaton, the plural form of automata, is used to describe the machines that move by themselves without possessing any electronic equipment. 


\subsubsection{Robotics and Links to Autonomy}

The basic information gained so far links the readers to a subset of explorations much closer to the real scope of this study: the essence of autonomy in the field of robotics. Definitely contradicting Asimov's first law of Robotics that states "A robot may not injure a human being or, through inaction, allow a human being to come to harm." [2], today's robots are more appropriate to the vision of Karel Capek who for the first time presented the "robot" 1 term invented by his brother Josef Capek to the society in his play Rossum's Universal Robots in 1921 [3]. In Capek's vision, rather than a society member restricted to some rules, humanoid robots were closer to some sort of slaves with no will-power except that of its creator as today's robots are. The line of a well-beloved poet portrays this assertion quite adequately: "The catastrophe of a robot is as far as the rusting of steel".

Returning to the definitions within the scientific limits of academic studies, robotics deals with design, manufacturing, and operation of robots and their autonomy with aims of executing industrial tasks as well as performing missions in hazardous situations. The control of robotic systems, software architecture, hardware and sensor equipment for feedback and information processing are in the extent of robotics. On the most basic level, robots may be claimed to have five major constituents: a body structure; a locomotion and actuation system for motion generation; sensors for receiving information about the states of the body and the surroundings; reprogrammable intelligence unit for the interpretation of the sensor data and the decision on what actions to take and power supply segments for sensors, actuation mechanisms and hardware units of decision.

In this sphere of robotics, autonomy roughly stands for the capability to accomplish assigned tasks in unpredictable or unstructured environments without having continuous guidance or intervention by the human operator. Fogel makes the definition of autonomy as "the ability to generate one's own purposes without any instruction from outside" while Clough generalizes it as "having free

\footnotetext{
1 The "robot" word was originally derived from "robota" which mean "corvee" or "serf labor" in Czech language.
} 
will" [4].

Interpreting this capability as the independence of control, Pfeifer and Scheier state that such depiction is nothing but a property of the connection between the designer and the autonomous robot [5]. Besides, the degree of autonomy, as will be discussed later for the focal point of this work, can be improved by factors such as self sufficiency, adaptation, learning, enhancement, embeddedness and evolution.

\subsubsection{History of Autonomy and Robots}

A brief introduction to the history of autonomy concept is most appropriate at this point so as to depict the diversity of the use of autonomous robots in many fields.

Beyond the initial examples of autonomy in the recorded history of humankind, Heron of Alexandria, a mechanics who lived between 10-85 AD in Alexandria of today's Egypt, can be counted. Particularly famous for documenting the first steam turbine, the aelolipile, Heron indited the descriptions of automated machines powered by mechanical and pneumatic means such as water and air. The automation related parts of his works were published in the Automatopoietica of the Teubner Series [6].

Then, the 9th-century scholars of Baghdad, the Banu Musa brothers, gathered their studies about automata and mechanical devices in "The Book of Ingenious Devices" [7]. Their works included a number of early automatic control approaches which included two-step level controls for fluids, an early form of discontinuous variable structure controls as referred from Otto Mayr's work at 1970 in Adamy and Flemming's survey [8]. Most significantly, the invention of conical valves and the implementation as in-line components into flow systems by the brothers were the first recorded use of automatic controllers as told by Hill in "Mechanical Engineering in Medieval Near East" [9].

Moving on to the later eras, it is worthwhile to touch on the genius of the idealized Renaissance human, Leonardo Da Vinci. The design of a humanoid 
automaton in the sketchbook of Da Vinci belonging to approximately 1495 was rediscovered in late 1950s by Italian historian Carlo Pedretti. After a replica was constructed faithfully according to the plans in the sketchbook to perform the tasks of standing, sitting, and independently manoeuvring its arms, this robot known as "Leonardo's Mechanical Knight" proved its functionality [10].

In the 18th century, Jacques de Vaucanson, a French engineer, presented his automata, Flute Player and the Digesting Duck. These efforts resulted in the crediting of Vaucanson with creating the first completely automated loom and the first robots in their true meanings [11].

Nevertheless, the most mysterious automaton of all might be the chess-playing mechanism "Turk" presented by Baron von Kempelen in Vienna, Austria in 1769. The attempts to respond to the questions raised about the fidelity of the smart automation of this mechanism still remain unsatisfactory. Whether a hoax or not, the Turk was an inspirational move for the foundations of the chess programming by Alan Turing in 1950s. Furthermore, chess programming has long been accepted as a major challenge for the evaluation of the abilities in programming due to the fact that the computer architecture should be capable of playing chess autonomously without human guidance, and, therefore, deserves to be mentioned [12].

\subsubsection{Works of Previous Century for the Development of Autonomy and Robotics}

The notion of automata resulted in works in the previous century that became the cornerstones for the developments of today's autonomy and robotics. Figure 1.1 illustrates primary works in these fields.

Developed by John Hammond Jr. and Benjamin Miessner in 1912, the "electric dog" was the first documented implementation of photo-tropic self-directing robots. If an electric light source was to be turned on in front of that robot, it would immediately move towards that source [13].

Miessner, who was working in the field of the wireless control of mobile torpedoes 


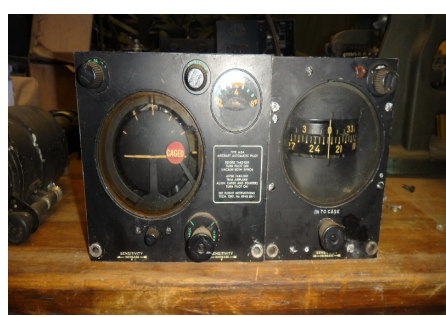

(a) Sperry Autopilot [15]

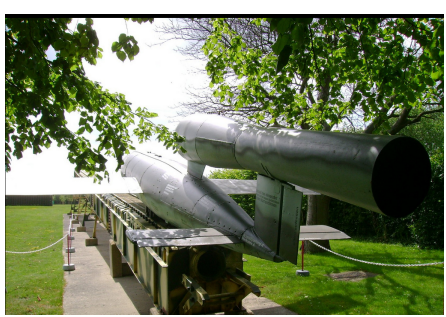

(b) V1 Buzz Bomb 16

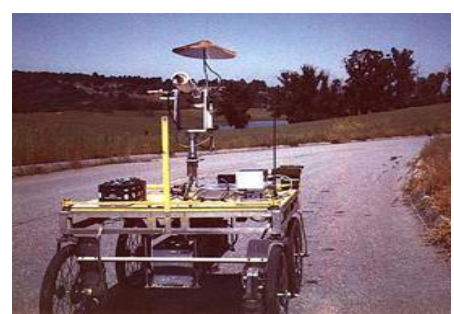

(c) Stanford Cart 17

Figure 1.1: Milestones in Autonomy

then, described the analogy for this control circuit and applied it to Hammond's dirigible torpedo. In his book, he also pointed out possible use of self-directed aerial and naval torpedoes as an intelligent weapon [14].

In 1912, a remarkable invention was achieved in the field of autonomous flight by Lawrence Sperry who developed the gyroscopic automatic stabilizing device based on the gyrocompass patented by his father, Elmer Sperry in 1908. Now, mostly remembered as Sperry Autopilot, this first example of automatic flight control systems was verified by US Navy pilots on a Curtiss C-2 flying boat on August 30, 1913 [18],[19].

Connecting a gyroscopic heading indicator and an attitude indicator to the hydraulically operated elevator and rudder, the gyro-pilot permitted the aircraft to fly straight and level on a compass course without an outstanding pilot effort considering the fact that aeroplanes of the decade were still in an unstable and underpowered structure [20].

During the First World War, two noticeable American developments were the autonomous flying bombs, The Navy/Sperry "Flying Bomb" and The Army/Kettering "Bug" (Liberty Eagle), which were indeed the first cruise missiles [21].

In 1922, the W.H.Tilford tanker of Standard Oil company crossed the Atlantic completely under the control of Sperry Gyro-Pilot which was the first time for a ship to travel over such distance with the guidance of an autopilot [22].

Containing a gyroscopic guidance system and a simple pulse-jet engine, German WW2 V-1 Buzz Bomb [23], the ancestor of all modern cruise missiles had been 
designed to deliver a warhead to targets with accuracy not more than that of the bomber aircraft of the time.

With the enhancement of embedded algorithms in controllers, the advancing technology of moving surface mechanisms and the presentation of radio-navigation aids, night flights and even flights under bad weather conditions were made possible. In 1947, a US Air Force C-54 Skymaster executed a transatlantic flight from Stephenville, Canada to London, England. Including take-off and landing, this flight was performed entirely under the control of an autopilot [24].

Envisioned in 1956 by George Devol and Joseph Engelberger, Unimate was installed on the assembly line in 1961 earning fame as the first industrial robot. The ingenuities of the robot arm were the removal of parts from die casting machines and welding tasks [25].

Clearly, the contribution of university research groups to the field of autonomy was inevitable and Stanford University's cart holds a well-regarded place in this history. Originally constructed by James L. Adams in 1961 to research about the problem of controlling a remote vehicle on Moon (e.g. a lunar rover) using video information from Earth [26], Stanford Cart was then rearranged as a robot road vehicle for various dissertation researches in Stanford. These studies include the predictive steering control [27], autonomous navigation on a road using lines [28] and indoor navigation using stereo vision [29]. What brings the cart project into the scope of autonomy is the fact that the project was surely the first leap into the concept of programmable mobile robots.

\subsubsection{Current Field of Use of Robots and Autonomy in Robotics}

Whether there exists a word that is sufficient in meaning to explain the current field of use of robotic technology, it should be apparent by current trends that the fields of use of robots ranging from land, sea and air to near and even interplanetary space, are not intended to be constrained within some boundaries. Figure 1.2 illustrates significant examples from these fields. 


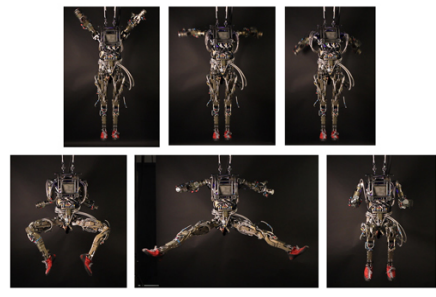

(a) Petman 30

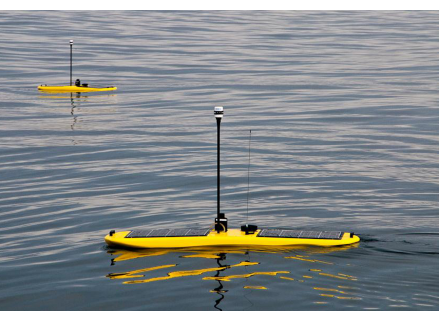

(b) Wave Glider 31

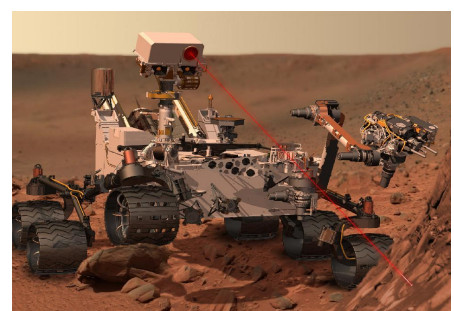

(c) Curiosity Rover 32

Figure 1.2: Robotic and Autonomy Examples from Current Fields of Use

The mobile robots in land, also known by the name, Unmanned Ground Vehicles (UGV), are widely employed for search and rescue, reconnaissance, military missions, and operations in hazardous environments such as mapping of the areas affected by radioactive fallout and explosive ordnance disposal (EOD). MVF-5 autonomous fire fighting robots by DOK-ING [33], iRobot Packbot with EOD capabilities [34], Toshiba 4-legged nuclear plant inspection robot [35] and developments by Boston Dynamics such as SandFlea [36], BigDog [37], PetMan [38], Atlas [39], LS3 [40] and RHex [41] are some examples of UGVs noteworthy to touch on. Among the endeavours to research autonomy on ground, the concept of autonomous car driving has especially been a research concern and was attempted in researches of University of Bundeswehr Munich, Stanford and Carnegie Mellon. Besides, a program named "Mobile Autonomous Robotics Technology Initiative" was initiated by SwRI [42].

Though not yet utilized extensively and effectively, robots are still exploring critical areas that are too difficult to reach for humans with aims of search \& rescue or scientific exploration. For instance, the emergence of underwater robots resulted in various maritime robotic applications intended for demining operations, submarine rescue, underwater repair work and deep water exploration. There are solely few products developed for these applications, some of which are Woods Hole Oceanographic Institute's ROV (Remotely Operated Vehicle)class robot Sentry, iRobot's AUV (Autonomous Unmanned Vehicle) Seaglider, Bluefin Robotic Corp.'s Bluefin-12 [43] and Liquid Robotics' AUV Wave Glider 44. 
The manner "autonomy" is inferred and used in aerospace engineering scope is not indeed quite further away from the aforementioned fields. The increasing emphasis on operations that require the ability to cope with the environment and dictations, has turned the concept of autonomy to remain a trending topic in aerial and space applications.

Particularly, most of the novel approaches in autonomous mobile robots appeared when a space-oriented research necessitated such an effort. Despite the common delusion that most of the spacecrafts including satellites and probes are not generally considered robots, these vehicles can safely be subject to this classification. The use of the robotic manipulator systems for purposes of grasping and moving objects constitutes support for the assertion of this diversion. Some primary examples are the Space Shuttle and Canadarm 1 on the International Space Station [45]. Moreover, these space vehicles provide astronauts with convenience as mobile work platforms.

The first artificial satellite in space was Sputnik 1 of USSR. Among other early attempts, lunar orbiters \& flybys and solar monitors of the Pioneer vehicle family of the USA and the lunar landers of the Soviet Luna vehicle family can be counted. 46]

More enhanced generation of robotic spacecrafts included the space probes of Venera 3 of USSR (the first man-made spacecraft to impact on another planet), Voyager 1 (the farthest human-made object from Earth), Deep Space 1 (the first spacecraft showing the feasibility of full autonomy) 47] and The Mars Exploration Rovers, by name, Sojourner [48], Spirit and Opportunity [49] of the USA.

The most recent state-of-art in space robotics are The European Robot Arm (ERA) [50], The Mars Science Laboratory and its rover Curiosity [51]. Especially, ERA is notable due to its capability of self-repositioning as well as automatic \& semi-automatic task performance.

Finally, autonomy of the aerial vehicles is the remaining discussion point under this section. In modern cruise missiles, self-navigation, supersonic travel and 
non-ballistic, extremely low altitude trajectory flight have been achieved. Nevertheless, cruise missiles cannot be delineated as autonomous. In the definition of automatic as "pre-programmed to do a task", these missiles having no free will to act are perfectly automatic. Perhaps, the most intriguing robotic platforms making use of autonomy are the uninhabited aerial vehicles (UAVs). Holding great promise for a variety of mission scopes, UAVs have reached widespread use. Exploitation of this potential has recently been widely available thanks to the advancements in autonomous flight technology which coherently provoked the rise of the UAVs. In performing dangerous military tasks such as combat, intelligence, surveillance and reconnaissance, UAVs prevent the possibility of putting the human lives into jeopardy. In addition to these duties in armed forces, UAVs show consistent efficacy for many civilian applications like scientific research, forest fire detection, geological remote sensing, agricultural use, pilot training, and even film making. Figure 1.3 illustrates UAV employment areas and Table 1.1 provides the names of several examples referred in the figure.

The dictations required by the functionality of an uninhabited aerial vehicle for different mission scenarios and weather conditions, and the availability of in- and out-of-flight changes depending on these dictations point out the significance of the implementation of the augmented autonomous control devices to these platforms. With the help of the developments in the redundant flight controls and sense-avoid mechanisms, the experience gained in UAV technology yielded to an analogous frequency in UAV utilization to that of manned aircraft. The usage of UAVs by troops revealed some advantageous results including less military casualties, reduced reaction time and workload during wars.

At the moment, the capabilities of military UAVs are broadly being researched with regards to the persistent visibility of the battlefield, the direct connectivity of UAV operators to ground forces, way-point navigation and automatic takeoff and landing [52]. Hence, it is not pointless to foresee that these robots will remain the owner of the skies in the near future. Several challenges remaining are the efficient training of personnel for unmanned aerial systems, higher comprehension of the integration of command and control and the optimality of the distribution of intelligence, surveillance and reconnaissance products. 


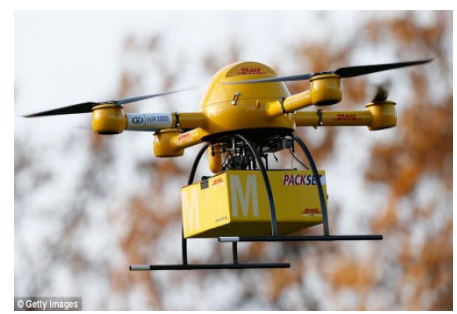

(a) Mikrokopter 53 .

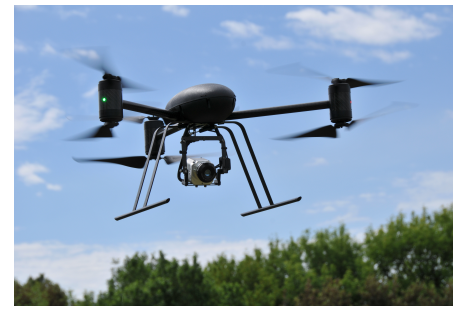

(d) Draganflyer X-6 [56]

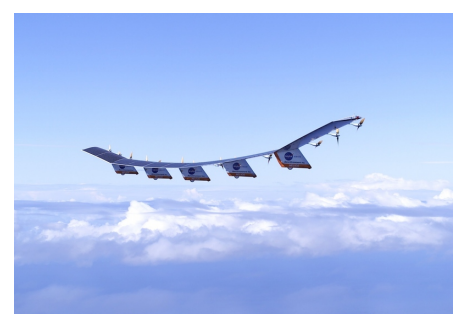

(g) NASA Helios 59]

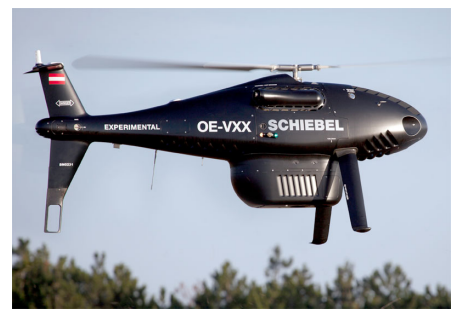

(j) Schiebel Camcopter 62

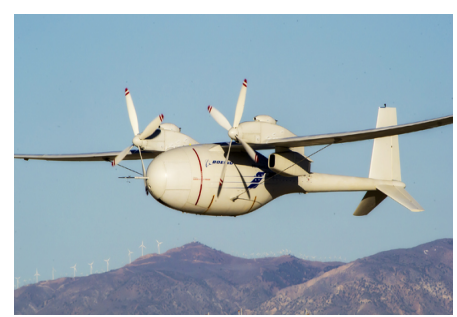

(m) Boeing Phantom Eye 65

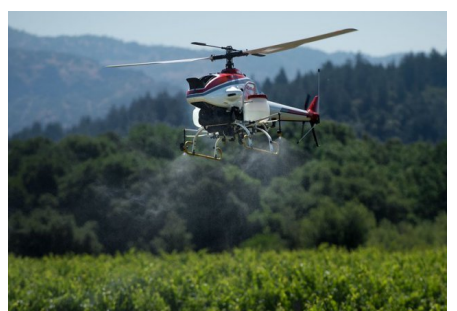

(b) Yamaha RMAX 54

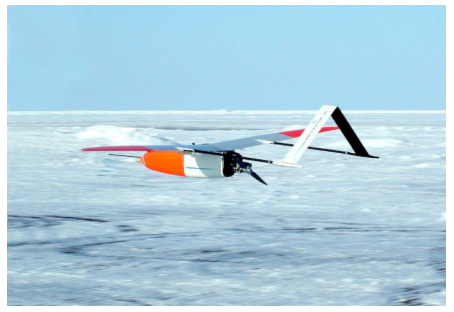

(e) AAI Aerosonde [57.

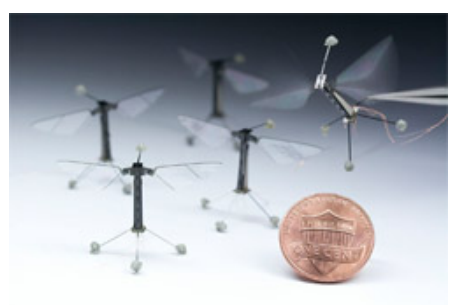

(h) Harvard Robo-Insect 60

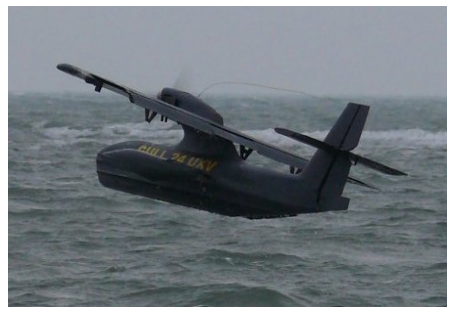

(k) Warrior's Gull UAV 63

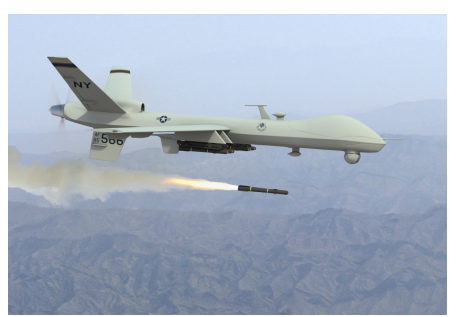

(n) MQ-9 Reaper 66

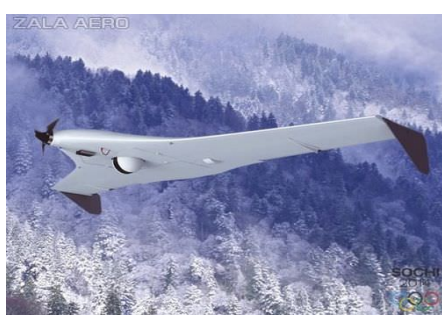

(c) ZALA 421-16EM 55]

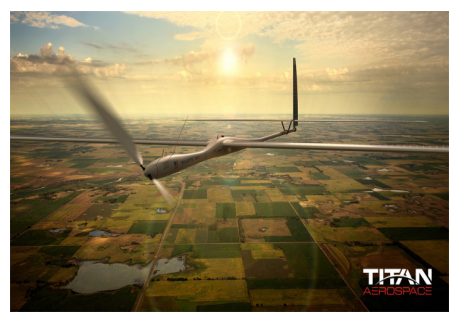

(f) Solara 50 Concept 58

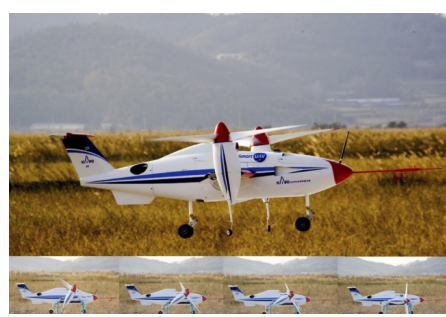

(i) KARI VTOL UAV 61

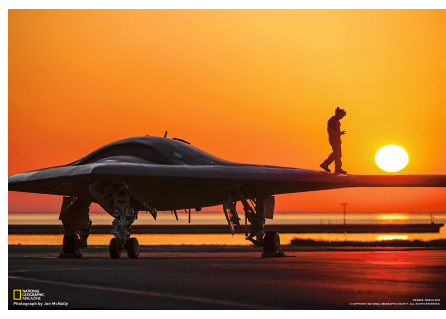

(l) NG X-47 B 64

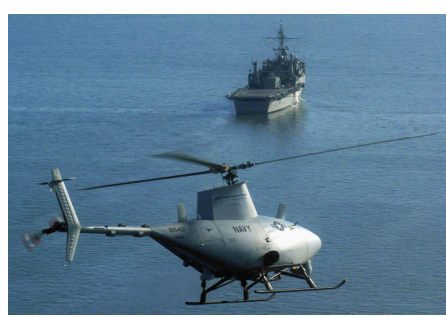

(o) MQ-8 Fire Scout 67]

Figure 1.3: UAVs and their Fields of Use 
Table 1.1: UAV examples

\begin{tabular}{ccccc}
\hline UAV Example & & Type & & Field of Use \\
\cline { 1 - 2 } Mikrokopter & & Quadcopter & & Transportation \\
Yamaha RMAX & & RC-Helicopter & & Agricultural Use \\
ZALA 421-16 EM & & Flying Wing & & Aerial Vision Capture \\
Draganflyer X-6 & & Quadcopter & & Aerial Photography \\
AAI Aerosonde & & SUAV & & Weather Data Collecting \\
Titan Solara 50 & & Solar UAV & & Atmospheric Satellite \\
NASA Helios & & Solar UAV & & Scientific Research \\
Harvard's Robo-Insect & MAV & & Miniature Robotics \\
KARI VTOL UAV & & VTOL UAV & & VTOL Tiltrotor Research \\
Schiebel S-100 & & RUAV & & Terrain mapping \\
Warrior's Gull UAV & Seaplane UAV & & Amphibious Operations \\
NG X-47 B & Stealth UCAV & & Carrier Based Combat \\
Boeing Phantom Eye & Liquid-Pow.UAV & & Advanced Intelligence \\
MQ-9 Reaper & HALE UCAV & & Surveillance-Combat \\
MQ-8 Fire Scout & RUAV & SA-Fire Support-Precision Track \\
\hline
\end{tabular}

\subsubsection{Levels of Autonomy in UAV Technology}

Contrary to the current misconception that robots elicit independent judgements resulting in uncontrolled operations, all autonomous UAV systems are supervised by human operators at a certain level, and their software embraces the boundaries of the actions and the ruling. In order to endorse this statement, however, there had existed no grading method for the autonomy level of operating systems in the literature before the previous decade. Therefore, in an effort to respond to the needs about acquiring a clear vision of where the current technological developments fit in terms of autonomy, there appeared various attempts to generate a common measurement tool. The principal motivation behind these inspections was that, with the help of these approaches, the planning phase could more accurately be completed and the progress of the works could easily be comprehended by the consortium of research labs, federal and management units, universities and industrial corporations.

The fundamental knowledge over the modelling of human-computer interaction 
was commenced by Sheridan in [68] where he suggested a 10-level scale. Extending the above model in 2000, Parasuraman et al. 69] summarized autonomy into "acquire-analyze-decide-act" function groups.

Apart from the intelligence metrics such as Turing Test [70], early approaches to propose autonomy metrics in robotic and aerospace systems were the MobilityAcquisition-Protection survival metric space by Los Alamos National Lab [71] and autonomy radar chart based on Three Dimensional (situational awarenesstask planning-mobility control) Intelligent Space by Draper Lab [72]. Combining the best sides of above approaches for an initial chart and Boyd's OODA (Observe-Orient-Decide-Act) Loop [73] to access enemy's decision loops, Clough [4] presented an autonomous Control Level Chart identifying 11 levels of autonomy in 2002.

Another advanced framework proposed was ALFUS (Autonomy Levels for Unmanned Systems) where the contextual autonomous capability was measured by weighting the score of various metrics from the perspectives of human independence, mission, and environmental complexity. [74. Due to the fact that all these suggestions were military-based and not focused on rotorcrafts, Kendoul developed the research-oriented framework ALFURS (autonomy levels for unmanned rotorcraft systems) as a better suit to rotorcraft unmanned aerial systems operating at low altitudes and in cluttered environments [75]. ALFURS utilizes Guidance, Navigation and Control capabilities of systems as autonomy functions and combines them with their corresponding external system independence, mission and environmental complexity. These enabling functions are indeed the core knowledge to perceive the level of autonomy. Table 1.2 illustrates a summary of this framework.

The perspective of any automatic control scheme can be described as the elimination of the human operator out of the control loop and the manipulation of the inputs by synthesizing algorithms with an aim to generate desired outputs. The automatic control of a flight system, in most general sense, regards the outputs as the torques or forces acting on the vehicle and the inputs as the commands for the motion of the actuation mechanisms on the vehicle. By introducing the laws 
into the controller, the aim is to achieve the control of orientation and position of the system and their respective time derivatives in the duration of flight.

Table 1.2: The Description of Autonomy Levels

\begin{tabular}{ccc}
\hline Level & Description of Autonomy Level \\
9 & & Swarm Cognizance and Group Decision Making \\
8 & & Situational Awareness and Cognizance \\
7 & Real Time Collaborative Mission Planning \\
6 & Dynamic Mission Planning \\
5 & Real Time Cooperative Navigation and Path Planning \\
4 & Real Time Obstacle/Event Detection and Path Planning \\
3 & Fault Tolerance and Event Adaptation \\
2 & Navigation without the Aid of an External System \\
1 & Automatic Flight Control Capabilities \\
0 & Remote Piloting by Radio-Control \\
\hline
\end{tabular}

The navigation can be represented as the procedure of authentically clarifying the position of the vehicle and monitoring the track of the planned route. For unmanned aerial systems, navigation includes research phases like data acquisition for sensing, analysis \& extraction of that data, evaluation of attained information about the vehicle and the surroundings as well as the objective of accomplishing assigned missions safely.

The guidance segment of autonomy intends to take the position of the cognitive operation of the intelligent agent. Located at the highest level, a guidance system receives inputs from navigation, decides and generates commands for the low level AFCS in order to ensure the completion of the assigned mission. The complements of a guidance system may include operation based planning, trajectory generation, decision making and reasoning.

Nevertheless, as stated in DoD Report on autonomy [52], such taxonomies are deceptive from both a cognitive science view and observations of actual practice since focusing on the machine rather than the human-system collaboration has engendered designs "disregarding overall resilient capabilities". The report sug- 
gests the participants of UAV autonomy-related projects to quit the deliberation on describing levels and embrace a framework based on design decisions for autonomy. Figure 1.4 shows these classes, the perspectives of cognitive science, mission dynamics and complex system trades space, respectively [52].

\section{FRAMEWORK FOR THE DESIGN AND EVALUATION OF AUTONOMOUS SYSTEMS}

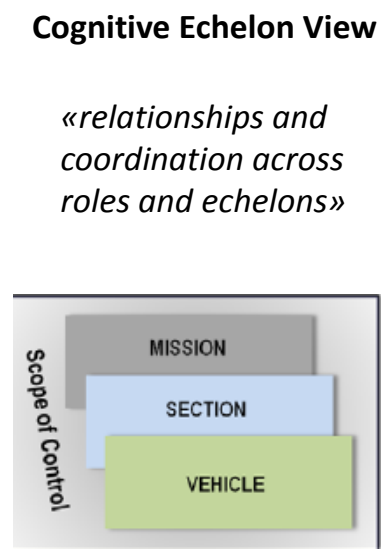

Mission Dynamics View

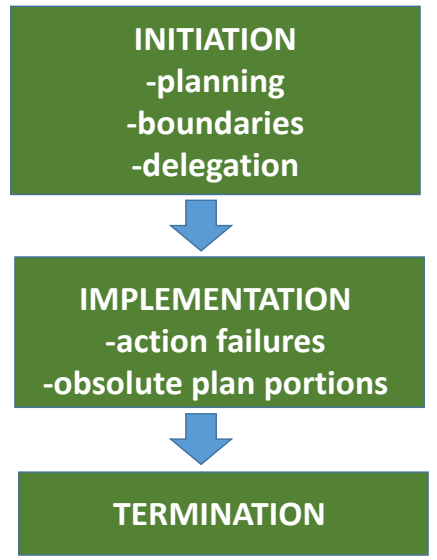

Complex System Trades Space

"design choices about how and where to inject autonomy»

-short vs. long term goals

-efficiency vs thoroughness

-centralized vs. distributed

-local vs global views

-optimality vs. resilience

Figure 1.4: The Perspectives suggested in DoD Report for Autonomous Systems

\subsubsection{Unmanned Rotary Aerial Vehicle Systems}

The superiority of unmanned rotorcraft systems over fixed-wing UAV systems especially comes into prominence when the operation requires a set of criteria mostly calling for the capabilities of the rotorcraft systems. Such capabilities may vary from vertical take-off and landing to hover and low-altitude flight. This fact may be reflected by missions of reconnaissance, surveillance, remote inspection or intelligence gathering that are based on mainly collecting vision over territories. Therefore, these missions necessitate low speed and hover flight to acquire clear vision from the on-board camera. Furthermore, vehicle manoeuvrability and robustness in the existence of disturbances come into question with the need to fulfil the purposes of air pollution monitoring, agricultural applications, film-making and even law enforcement via UAVs. In this manner, the abilities of 
rotorcrafts consolidate their domination over fixed-wing platforms. Many aerial vehicles including quadrocopters, micro aerial vehicles, radio controlled-models, autonomous military platforms and even full-scale helicopters transformed to perform missions as "uninhabited" can be gathered under the title of unmanned rotorcraft platforms.

\subsection{The Concept of Quadrocopters}

This study includes the employment of a quadrocopter platform as test-bed. This subsection includes the main points of the concept of quadrocopters as well as their history and state-of-art works about them in literature.

\subsubsection{History}

When the idea of the Helical Air Screw popped up in Da Vinci's mind, it was no later than the last decade of the 15th century [76]. Describing a coaxial helicopter and how to operate it, Ponton d'Amécort had spelled the word "helicopter" for the first time by combining Greek words "elikoeioas" (spiral) and "Pteron" (wing) [77]. Fortunately, the attempts by Forlanini in 1877 [78], by Breguets \& Richet and by Cornu in 1907 [77] led to the birth of the helicopter concept. In particular, Breguets and Richet's Gyroplane No:1 had accomplished the first manned helicopter flight from the point of view of that era. By looking at the structure of the gyroplane, this uncontrolled flight can as well be interpreted as "the first quadrotor flight" using today's jargon.

A quadrotor can be described as a UAV lifted and directed by four rotors. The fact that a quadrotor attains its lift from its rotors leads to its classification as rotorcraft. As opposed to most helicopters, propeller blades of quadrocopters, in general, are not pitch-varying. The control of the motion is achieved by adjusting the relative angular speed of each rotor which, in turn, changes the thrust and torque generation, and thus attitude in desired directions.

When the history of the quadrocopters is investigated in terms of application and 
design, the presence of two generations is obvious. First generation quadrotors were developed for passenger or load transportation and recorded as the first to accomplish vertical take-off and landing among heavier-than-air vehicles. Nevertheless, further exploring this concept, many aspects of these attempts resulted in serious handicap about the future use of these vehicles. In addition to the inadequacy in the performance of the prototypes from 1920s, the drawback of the designs from 1950s was the overwhelming pilot work load due to the insufficient stability augmentation of these later decade vehicles. Some of these early works are shown on Figure 1.5 .

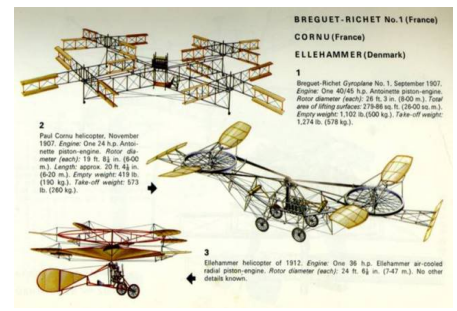

(a) Early Helicopters

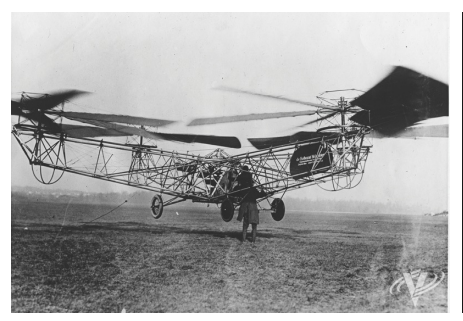

(b) De Bothezat's Quad 79]

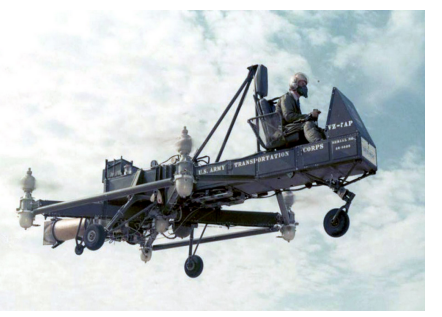

(c) Curtiss-Wright VZ-7 [80]

Figure 1.5: First generation of rotorcrafts and quadrotors

\subsubsection{Reason of Interest for Quadrocopters}

Excluding the preliminary efforts, the quadrocopters from the present-day are perceived as tools for academic studies regarding flight dynamics and experimentation on the algorithms for control, guidance and navigation. These studies have recently been so diverse and excessively widespread that quadrocopters are nowadays being used for purposes like visual inspection, target-tracking, and scouting.

Distinctive characteristics of these flying robots make them favourable test-beds against comparable classes of helicopters. To commence with, conventional quadrocopters do not have mechanical connections to vary the rotor blade pitch angle while spinning. This quality is a simplifying factor in design and maintenance. With their varying size and capabilities, the quadrocopters can be operated for miscellaneous task definitions in various environments from indoor 
to harsh wind conditions. The non-linearity and agility observed in the dynamics of these vehicles present a momentous challenge to test many controller approaches that will be discussed in the next subsection.

Furthermore, the convenience in the utilization of quadrocopters expedites the verification of the developed methodologies through real-time experimentation. Regarding such a manner, testing the capabilities of schemes pertinent to the existence of disturbances or uncertainties becomes viable via a quadrotor testbed that is highly susceptible to this kind of disturbances in its nature. For that reason, these types of control problems are excellent opportunities for developing and testing new ideas in autonomy. A key aspect to developing such stabilization techniques are the measurements of the position and the orientation of the air vehicle. These measurements depend on the effective use of the integrated sensors with the electronic control system.

By assuming the structural frame of a quadrotor as a rigid body, the state variables can be characterized with three translational and three rotational degrees of freedom (DOF), i.e. a total of 6 DOFs and 4 control inputs. This makes quadrocopter an under-actuated mechanical system that is a system having fewer control inputs than state variables. These inputs represented by four propeller-motor dualities makes it impossible to attain a desired set-point for all DOFs. Nevertheless, thanks to its unique structure, a controller may sufficiently perform to allow the quadrotor to reach certain height and attitude. This is achieved by creating the four best controllable input variables that in turn forms four fundamental movements, which are descent/ascent, pitch, roll and yaw. It is essential to categorize the rotors on a quadrotor into two groups with each group rotating in the opposite direction of the other group to eventually have a controllable vehicle in various directions. In addition, since quadrotor has a symmetric structure in 2-axis, the analysis for pitch and roll axes can be perceived as the same. The dynamics of a quadcopter appears as couplings such as pitch angle \& x-direction coupling and roll angle \& y-direction coupling. Such coupling can be utilized for position control. Figure 1.6 illustrates the motion primitives of a quadrotor test-bed. 

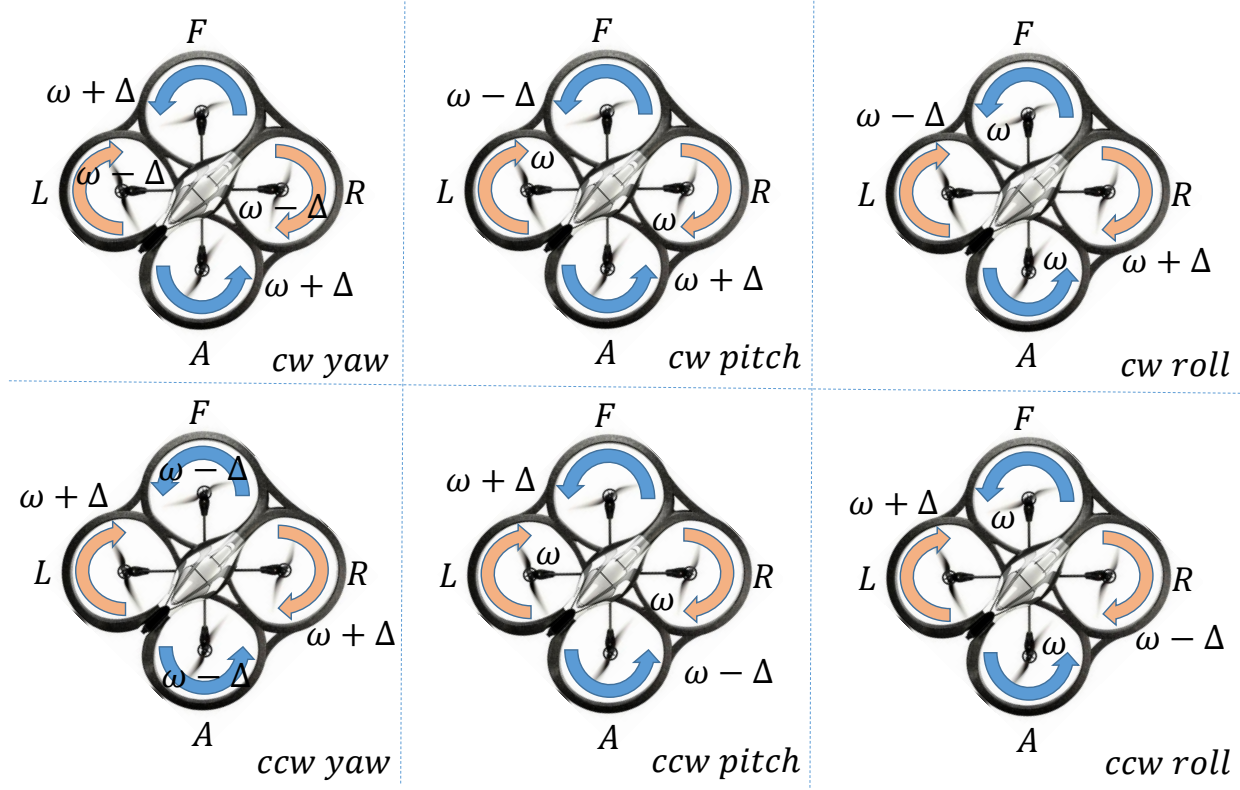

F: Front
A: Aft
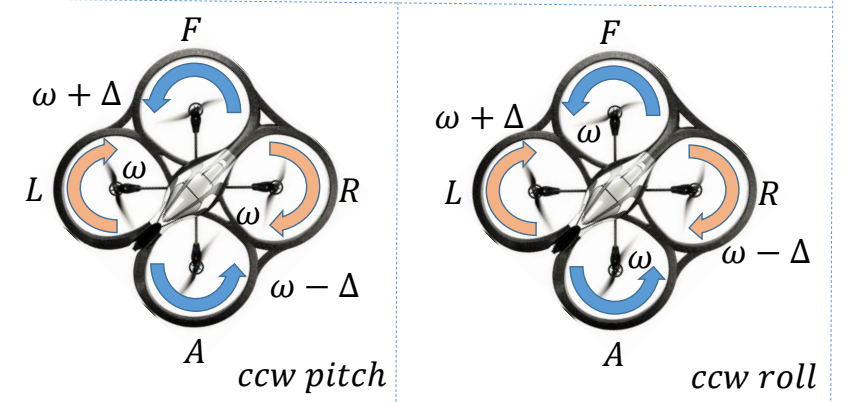

R: Right

L: Left

Figure 1.6: Motion Primitives for a Quadrotor

Designing an efficient controller for an aerial platform requires high-fidelity modelling that might include rigid-body dynamics, force-torque generation mechanism, the modelling of actuator dynamics and the integration of rotor dynamics and aerodynamics. The model of quadrotor dynamics represents an expression that relates the commands and system responses. In order to construct a basis for a modelling phase, a quadrocopter is assumed as a rigid body of cross shape composed of four arms in general. The rotors on the tip of each arm of this structure are modelled to produce thrust and torque vectors. Since the vectors generated by the propeller motor combinations are generally perpendicular to the arms of the platform, the orientation of each thrust force is also perpendicular to that platform plane. These thrust and torque vectors are converted to the resultant force and moments experienced by the body through geometrical relations and computations. This forms the mechanism of force and moment production. Extending modelling phase with the actuator dynamics denoted by the brushless electric motors and the rotor aerodynamics \& dynamics represented by the use of simplified blade element and momentum theories may increase the accuracy and fidelity. 
Obtaining dynamic models may involve using various techniques such as the firstprinciples techniques or system identification. For example, the first principles techniques involve obtaining the mathematical equations of motion using the laws of Newtonian physics and aerodynamics. The next step in the procedure is the simplification of these models through the use of Taylor series expansion, feedback linearization or decoupling.

\subsubsection{State-of-Art and Literature Survey for Quadrotors}

For researchers from industry and academic society, quadrocopters have literally been a trending topic in the last decade. In addition to the elements mentioned in the previous section, the list of challenges may be extended with the comprehension of low speed aerodynamics, use of lightweight frames and electronic/sensor equipments, the assessment of robustness in flight and the integration of computational models in feasible manners.

As for other uninhabited aerial platforms, quadrocopters do also depend on the sensor units integrated. While the attitude sensing can be achieved by means of inertial sensors including gyroscopes, accelerometers, magnetometers, compass and inertial measurement units, estimation for pose, velocity and height and perception of the system are accomplished by the utilization of global navigation satellite systems, altimeters, laser range finder, infra-red and ultrasonic sensors [81.

General structure of a controller scheme for a quadrocopter is composed of an attitude controller at the most interior loop and a position controller covering it, together forming the core of motion control and other outer loops related to navigation and guidance purposes. These sub-parts closed over each other may be coupled or decoupled in compliance with the frames of control loops. Such controller algorithms examine the effects of computational feasibility, flight performance and robustness against environmental challenges as well as the establishment of motional boundaries of the system on the system dynamics during real-time experimentation. According to Kendoul, methodologies for the control of rotorcraft systems can be categorized into three branches: linear, learning and 
model-based [75].

Linear Flight Control is the most widely endorsed method with its unambiguous design and implementation routines. Not to mention many tools for gain scheduling and analysis for robustness and performance, linear controller action has a prosperous past use in a broad range of tasks regarding aerospace systems. On the other hand, with no severe unease for saturations, such flight controllers undergo performance degradation when out of nominal conditions or during aggressive manoeuvres. Other drawbacks are the difficulty in theoretically proving the asymptotic stability of the complete closed loop and the failure in using full bandwidth and dynamics of the system.

The work of Pounds on the PID controller with full flapping model and the linearization around hover state 82 and Bouabdallah's discussion of PID and LQ control techniques applied to an indoor micro quadrotor [83] are only two of many examples under the title of linear flight controller methods for quadcopters.

Falling within the robust control theory, $H_{\infty}$ method synthesizes controllers achieving stabilization with guaranteed performance against uncertain parameters and unmodeled dynamics. The disadvantage of the necessitated level of mathematical comprehension and the exceptional modelling of the system is compensated by the applicability of this method to problems concerning multivariate systems with cross-coupling between channels. In [84], Mokhtari made a simulation study regarding uncertainties, disturbances and noise accumulation for a mixed form of robust feedback linearization and linear $G H_{\infty}$ controller.

Among linear tools, the gain-scheduling-type techniques contain the modelling of non-linear dynamics as the combinations of simplified linear models. By decoupling the complexity of the trajectory planning phase and the update rate of the control input for the Stanford Testbed of Autonomous Rotorcraft for Multi-Agent Control (STARMAC) platform, Hoffmann developed an algorithm for autonomous trajectory tracking and generation which produce dynamically feasible sub-optimal trajectories at high level [85]. This linear path tracking controller combines the piecewise PI and PID structures having feedback, respectively, as error rate along the track direction and as error itself in the di- 
rection crossing the track. The basis of the attitude controller in this work that takes the second derivative of error into account by adding another zero to the classical PID structure can be found in the previous work of the author [86].

Using hybrid decomposition and reachable set theory, Gillula et al. achieved successful experimental results for the purposes of back-flip manoeuvre and collision-avoidance for multiple vehicles [87]. The motivation of this work is the idea of modelling the systems possessing complicated non-linear characteristics as a compilation of simplified hybrid modes for specific regions of operation as well as the transition between these modes. Safety and performance are ensured by reachability analysis, which draws on a distributed optimal switching control strategy derived from a dynamic game formulation.

When robustness to unmodeled dynamics or external disturbances are taken into consideration in order to grade controller options, model-based non-linear controllers outperform linear techniques by attaining tracking accuracy over a wider flight envelope. The number of experimental works, rigorous implementations and extensive flight tests of non-linear control algorithms are yet claimed to be "inadequate" [75]. Commonly utilized to control non-linear systems, "feedback linearization" involves the use of non-linear transformation techniques to convert the exact states of the system into an equivalent linear system dynamics. To ensure the equivalence of the system of interest and the system attained at the end of the operation, this transformation must be a "diffeomorphism", that is, an invertible function mapping one differentiable manifold to another so that both functions maintain the property of being smooth. Such an approach makes linear tools available for application and subsequently revert to the original coordinates via an inverse transformation. A typical case of feedback linearization is the well-known "dynamic-inversion".

Intriguing studies conducted by GRASP Lab of University of Pennsylvania included non-linear controllers based on dynamics inversion technique. A control algorithm for aerobatic/aggressive trajectory tracking and an approach to synthesize 3-D trajectories were described by Mellinger in [88]. The trajectories satisfy the constraints especially in tightly restrained indoor environments, guar- 
antee safe travel and keep optimality through a series of way-points in the set of positions and orientations by minimizing cost functions attained from the norm of the snap. Benefiting from mixed integer quadratic programming techniques, Kushleyev described the architecture and software for coordination of a group of quadrotors relying on external localization system for pose estimation during their flight through known 3-D environments with obstacles [89]. Several other works by the group contain algorithms for robust perching \& landing [90], aerial grasping and manipulation [91].

In [92], for the design of a cascaded non-linear inner/outer loop control structure, Kendoul used partial passivation and dynamic inversion techniques and combined laws for subsections of the system. The fidelity of this controller was verified in tests of attitude tracking, automatic take-off and landing, longdistance flight, way-point navigation, and spiral trajectory tracking [92], and vision-based flight research [93].

Especially, adaptation and robustness modifications in non-linear control methods have unprecedented potential against modelling errors and uncertainties. The research by Nicol focused on a new adaptive neural-network control architecture for quadrotor stabilization that surpassed the e-modification and deadzone techniques through simulations with wind disturbances. This method stems from Cerebellar Model Articulation Controller Neural Network methods in training approximation weights online [94].

The paper by Kendoul et al. 95] included the enhancement and the implementation of a non-linear indirect adaptive controller for vision-based flight of a quadrotor. The duo of incorporated adaptive visual odometer and flight controller resulted in the extension of optic flow-based control abilities to complex navigational tasks such as accurate hovering, arbitrary trajectory and target tracking. In [92], the authors augmented their work with an observer that provided the estimation of the visual unknown scale factor on-line via the sensor fusion of optic flow and IMU measurements and justified this upgrade in autonomous flights.

Another proposition for adaptation is the so-called Model predictive control 
(MPC) in which an explicit model of the system is employed to predict the future output response, and the minimization of the tracking error is accomplished by solving an optimality problem over a future horizon, due to which the method is also known by the name Receding Horizon Control. In the simulation study carried out by Raffo [96], the hierarchical scheme brought the concept of MPC and non-linear $H_{\infty}$ control for path following and rotational stabilization problems of a quadrotor respectively.

The back-stepping strategy was evaluated by many researchers under the title of adaptive control. This technique aims at recursively constructing the feedback control law and the stability analysis of closed-loop properties was conducted by Lyapunov Theory. Altuğ et al. introduced the stabilization and output tracking control through visual feedback using two cameras so as to estimate the full pose of the vehicle [97]. The methods utilized in this study are modebased approach with feedback linearization and a variation of the back-stepping technique. Guenard et al. [98] used back-stepping for image-based visual servoing of a quadrotor. Investigating alternative approaches such as back-stepping, sliding-mode [99, LQR and PID techniques [83] on his earlier works, Bouabdallah finalized his decision for altitude, attitude and position control by choosing the so-called "integral back-stepping" [100.

For quadrocopters, similar to other vehicles belonging to the class of small UAVs, actuator saturations which may restrain the availability of viable regions of operation and provoke induction of instability are design issues that should be handled with care, especially in specific cases of aggressive motion generation and unexpected disturbance exposure. Theorized by Johnson and experimented on Georgia Tech's well-known adaptive flight controller for RUAS [101, the pseudocontrol hedging concept deals with actuator limitations and the behaviour of system dynamics in those cases by hiding actuator dynamics from the plant. A good example of the implementation of the pseudocontrol hedging method to a quadrocopter-stabilization problem was [102].

Apart from all these methodologies, Castillo studied a non-linear controller extended with nested saturations in [103] and combined backstepping technique 
with extension of saturation functions in [104] to stabilize the attitude dynamics of a quadrocopter. In the former paper, the accumulation of actuator saturations were prevented by bounding control inputs and angular accelerations, finally attaining a low-performance but global stability in the existence of input saturations. The latter work contained comparison of the proposed controller in terms of transient performance with conventional PD methods. Benallegue's simulation study [105] was also a subset of model-based techniques. The control law was composed of a robust feedback linearization-based controller and a high-order observer employing sliding mode to estimate leverage of uncertainties and disturbances.

The non-linear tracking controllers developed by Lee for the problems of velocity, position and attitude tracking of the quadrotor vehicle make use of the special Euclidean group SE(3) for flight modes with almost global closed loop properties and robustness to mode-switching [106]. The purpose of using this special coordinate-free group was to avoid the singularities of Euler angles and the ambiguities of quaternions in representing attitude. The versatility of this approach had already been illustrated by various examples including recovery from being upside down [107].

Allowing direct mapping between data and actuator changes and providing fast reactive behaviour, the learning based control which is the last branch to discuss in the context of this taxonomy is model-free and flexible for implementation. There exist well-known learning-based techniques like fuzzy logic, Neural Networks, Markov Decision Processes and human-operated training. However, the stability and robustness analyses of this approach are difficult since not adequately extensive experiments over a variety of scenarios are available when compared to other methods. It is particularly sensible to teach the vehicles complex manoeuvres via human-based learning methods.

Montgomery used learning control to "teach helicopters by showing" [108] and Sugeno performed intelligent control of an unmanned helicopter based on fuzzy logic [109]. Coza proposed a new robust adaptive fuzzy control method for stabilizing the states of a quadrotor. As opposed to the e-modification requir- 
ing performance trade-off to prevent fuzzy membership center drifts during the activation of the update rule, this work prevents such drifting effectively [110].

Having constructed an overview about the background of control strategies, what is left to discuss in literature is the studies regarding higher autonomy capabilities, namely, state estimation, environmental perception, situational awareness, cooperation and coordination.

In literature, tremendous amount of research has been conducted concerning the problem of navigation and state estimation for quadrotors. Especially, efforts of eligible research labs about vision-based state estimation of quadrocopters were remarked worldwide. Such works emphasize the influence of the coordinated use of on-board sensors such as IMU and GPS with vision-aided navigation on situational awareness. Similar to the utilization of real-time motion capture systems in film featuring, VICON, a digitally optical motion capture system, has recently been broadly used by contributors at MIT's ACL, UPenn's GRASP Lab and ETH's Flight Machine Arena for experiments of indoor flight navigation and cooperation. As mentioned before, an early attempt to achieve this aim was the introduction of visual feedback by dual cameras for the stabilization and the estimation of the full position by Altug [97.

Aiming at autonomous navigation in unstructured and unknown indoor environments only based on on-board sensors and without the initial knowledge of a map, Achtelik et al. [111] made a comparison of laser range finder and a stereo camera. Noteworthy points from this study were the fusion of visual estimation and IMU data using an EKF, the feature tracking, 6 DOF motion estimation using least squares algorithm, non-linear motion estimation by bundle adjustment over a window of consecutive frames and flight control based on visual odometry estimates. In addition, the studies on visual odometry by Ahrens [112]; vision-based target tracking by Guenard [113]; Terrain Relative Navigation by Courbon [114]; structure from motion localization and mapping by Kendoul [95] and bio-inspired optic flow navigation by Conroy [115] lie within the boundaries of vision-based odometry.

Among problems concerning perception, there exist works about target detec- 
tion, target tracking and obstacle avoidance by simultaneous mapping and planning. Under the RAVEN project by MIT-ACL researchers, Bethke constructed a cooperative vision-based tracking algorithm for cooperation. This algorithm benefits from optimization techniques and Kalman filtering for target localization and target state estimation. An experimentation was achieved with two quadrotors and a target RC car [116]. As a part of another project from MITACL, Bachrach [117] developed real-time SLAM and SMAP algorithms for the mapping and exploration of unstructured and unknown GPS-denied environments in addition to the planning of obstacle avoidance for aimed navigation. Pose correction and occupancy grid updating of this LIDAR-based perception were achieved through the fusion of feedback data from LIDAR, stereo-vision and IMU and the use of Extended Kalman Filter and GMapping SLAM algorithm.

In an attempt to respond to the "situational awareness" requisition of autonomy problem, path planning algorithms making use of visibility graphs [85], probabilistic road mapping [118] and optimization [119], and probabilistic planning algorithms for tracking targets in the presence of uncertainties in target pose [120] were presented.

The demonstrations on the Stanford Testbed of Autonomous Rotorcraft for Multi-Agent Control (STARMAC) platform by Hoffmann [121 included two decentralized cooperative collision avoidance algorithms which were successively dependent upon optimal control and reachability analysis; non-linear, non-convex optimization and Nash bargaining cost metric. As stated before, the work by Gillula [87] was used to design collision avoidance algorithms for multiple vehicles as well.

In UPenn's GRASP Lab research about load transportation via the coordination of three quadrocopters [122, Micheal utilized the "inverse of direct kinematics problem" based on a mathematical model that includes the kinematic constraints and the mechanics underlying stable equilibria of the whole under-actuated system. The designed flight controller was advanced by a simple potential field algorithm to avoid collisions and the state estimation was achieved by using a 
VICON motion capture system.

Another active research field about quadrotors is multi-vehicle task assignment. Such an allocation study conducted in RAVEN project includes mission planning, decision making, task distribution and safe cooperation. After Bethke modified the algorithm for receding-horizon task assignment at MIT with his cooperative vision-based perception algorithm, an outstanding series of experiments were achieved. The new algorithm was specifically concentrated on the health management problem at the task level by selecting the optimal sequence of tasks for each UAS through optimization. In these multi-vehicle tasks, an unknown number of UGVs and three RAVEN quadrotors were employed for the experimentation of persistent and cooperative search and track, detection and estimation [116]. Some other works by this group are consensus based auction and bundle algorithms for decentralized cooperative task allocation in [123], variable pitch control in [124] and automated battery management system for enabling multi-agent persistent missions in [125].

Final part of the introduction is dedicated to the Flight Machine Arena projects at ETH Zurich which received a tremendous boost worldwide.

Hehn et al., in an effort to investigate the performance benchmarking problem, applied Pontryagin's Minimum Principle to a 2-D 1st Principle quadrotor model to find time-optimal trajectories for given initial and final conditions under the feasibility dictations by dynamical limitations. In the scope of the paper, useful lower bounds attained for manoeuvres were compared to understand theoretical potential of a control strategy and the influence of design parameters on performance assessment as well as the flexibility of these potentials for various other manoeuvres [126].

In a later work [127], targeting at minimizing the computational complexity in algorithms for efficiency, Müller presented a trajectory generation algorithm that excludes as much jerk trajectories as possible and includes an a-posteriori efficient feasibilty verification. The algorithm was based on an implicit feedback controller algorithm similar to MPC. The algorithm was successfully experimented on the problem of hitting a ball with a racket attached to a quadrotor. 
The time to calculate and apply the trajectories was on the order of miliseconds on a standard laptop.

Furthermore, Schoellig merged state-of-art convex programming optimization methods with classical model-based optimal filtering methods and formalized learning algorithm that exploits the experience attained from repetitive executions. The method uses a-priori knowledge about the system and adapts feed-forward input signal coming from the data-based update rule [128].

The following figure 1.7 includes photos from some of above works.

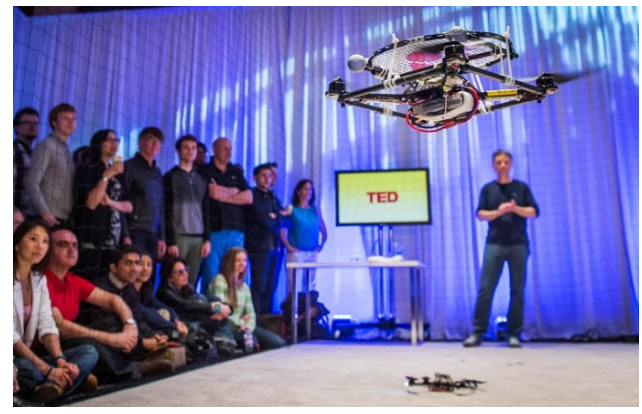

(a) TED Talks Photo from ETH-Z Demo

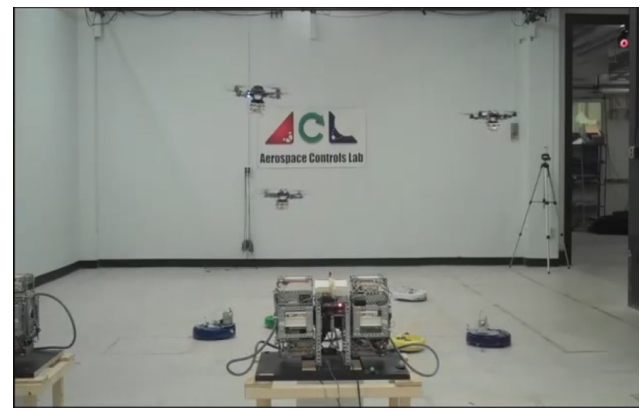

(c) Persistent Search and Track at MIT ACL

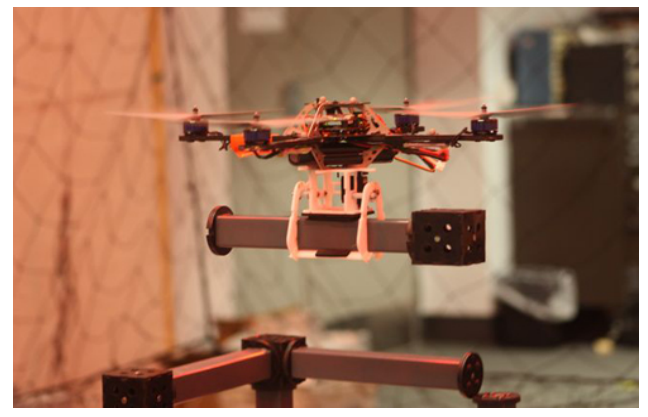

(b) Cooperative Transportation by UPenn

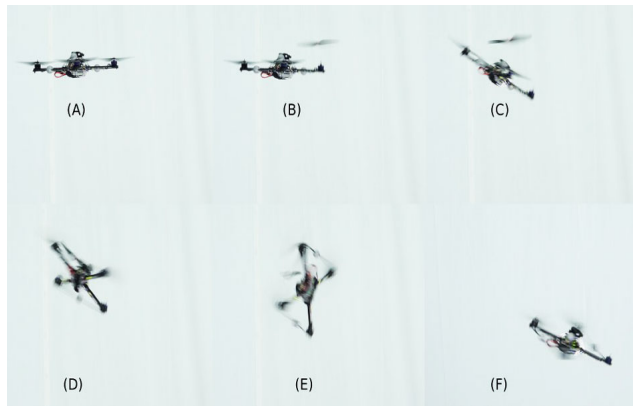

(d) Fail-safe Algorithm by ETH-Z

Figure 1.7: Research on Quadrocopters

\subsection{Motivation, Objectives and Content of This Thesis}

This study aims at the modelling and control of an aerial platform named as quadrocopter. Without leaving the questions unmarked about the concepts of performance and robustness, gaining knowledge on various control methods and the construction of attitude control structures via these is another motiva- 
tion. This work, perhaps most significantly, emphasizes on the fidelity of the adaptation in control strategy and also discusses the possiblity of robustness modifications to such adaptation procedures.

Chapter 1 makes a brief introduction about the topics of autonomy, control, UAV flight and literature survey about the quadrocopter research in academia.

Chapter 2 constitutes the modelling part and includes the physics background for the platform dynamics. The descriptions include the fundamentals of the system dynamic modelling, geometry and propeller dynamics, rigid body dynamics and attitude dynamics.

Chapter 3 deals with the controller strategies and presents the background of these techniques. The chapter starts with the methodology of how to apply the concept of the Non-Linear Dynamic Inversion which is actually a branch of feedback linearization to the current platform. The construction of trajectory generating reference model and its error dynamics are also discussed. The techniques of Pseudo-Control Hedging, Model Reference Adaptive Control and Integral Backstepping are explained broadly with their theory. As the last point in this chapter, the background of the robustness modifications to MRAC and the Lyapunov analysis for these MRAC varieties are also provided.

Chapter 4 includes the simulation results and discussion for the controller options described in Chapter 3. The performance and convergence of the controllers based on the mentioned methods are investigated in the presence of no noise, noisy measurements, parameter uncertainty and unknown disturbance. For these simulations, two types of inputs, namely step and sinusoidal, are used. Eventually, the necessity of adaptation is deemed inevitable although other methods may surpass the adaptive controller in terms of performance and minimized error especially in the cases without parameter uncertainty and unknown disturbance. The norms of these state error vectors are also analyzed. Studies about simulations with various step size values, various fized-step ODE methods, different levels of unknown disturbances and uncertain parameters are also conducted in order to see the sensitivity of the adaptation against these criteria. 
Chapter 5 contains discussion about the adaptation gains and robustness modifications to the adaptation. The influences of dead-zone, $\sigma$ and $e$-modifications to MRAC and the resulting effect in the system outputs are explored. The convergence of the adaptation gains are discussed with regards to the modification type. The performance and robustness of unmodified MRAC is also discussed with respect to the other extensions.

Chapter 6 concludes all the work briefly with the main points and the aims achieved. The suggestions for future work are also included in this section. 


\section{CHAPTER 2}

\section{MODELING}

\subsection{System Dynamics}

Understanding the reactions of the quadrocopter system to given commands that are directly related to the changes in the states is critical and requires a realistic description of the system model. The starting point of any controller design process is the analysis of the dynamics of the system to be controlled. This section summarizes the perspective behind the construction of the system dynamics of the quadrotor model.

Figure 2.1 indicates the axis definitions and free-body diagram to be used for modelling and control of system dynamics [129]. The coordinate systems shown in the figure are respectively earth and body axes. The body axis is defined by $x_{b}, y_{b}, z_{b}$, whereas the Earth axis is described with $x_{E}, y_{E}$ and $z_{E}$.

As stated in introduction, the dynamic model of a quadrotor presents high nonlinearities and strong couplings between its segments. The platform is assumed to be a rigid-body evolving in 3-D space generating force and torque vectors. The modelling can be divided into four subgroups for high model fidelity:

1. Force and torque production

2. Rigid-body dynamics

3. Rotor aerodynamics and dynamics

4. Actuator dynamics 


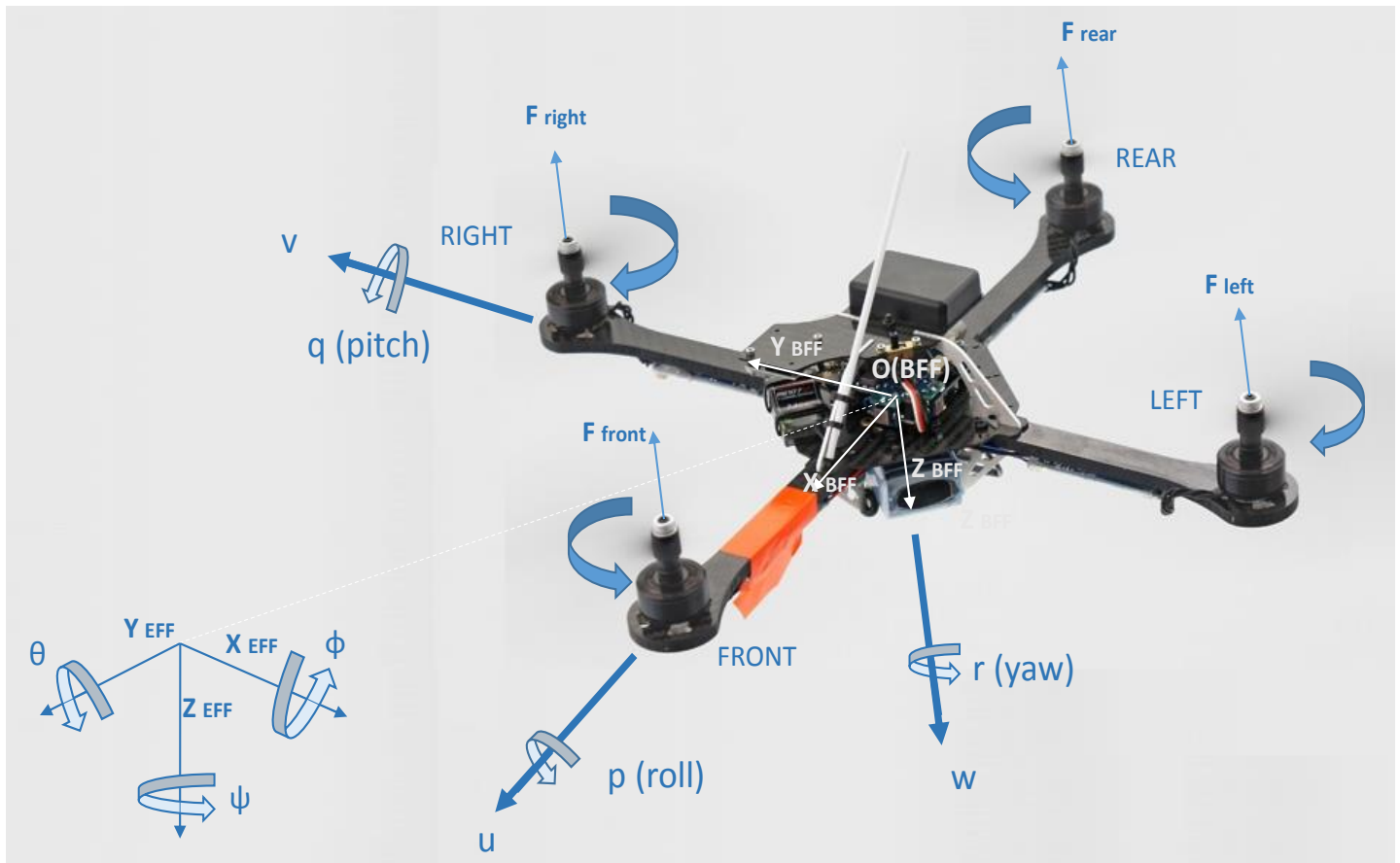

Figure 2.1: The Axis \& Movement Definitions for a Quadrotor

\subsection{Geometry and Propeller Dynamics}

This part includes the calculations of the resultant force and torque vectors experienced by the center of mass of the rigid-body. These force and moment vectors depend primarily on the thrust and torque vector produced by each rotor and the orientations of these vectors, propeller and motor dynamics, and geometrical properties of the rigid body.

\subsubsection{The Influence of Propeller Force on Body Center of Mass}

The computation of the body-fixed moments and the sum of forces in the $z_{B}$ axis of body frame are straightforward. This can easily be achieved through geometrical and axial organizations of values and vectors of thrusts generated by propellers. As discussed before, the pairs rotating in clockwise direction change roll attitude while the pairs rotating in counter-clockwise direction have effect on pitch attitude. The following matrix calculates the moments in each direction through the cross multiplication of the vectors that state the distance 


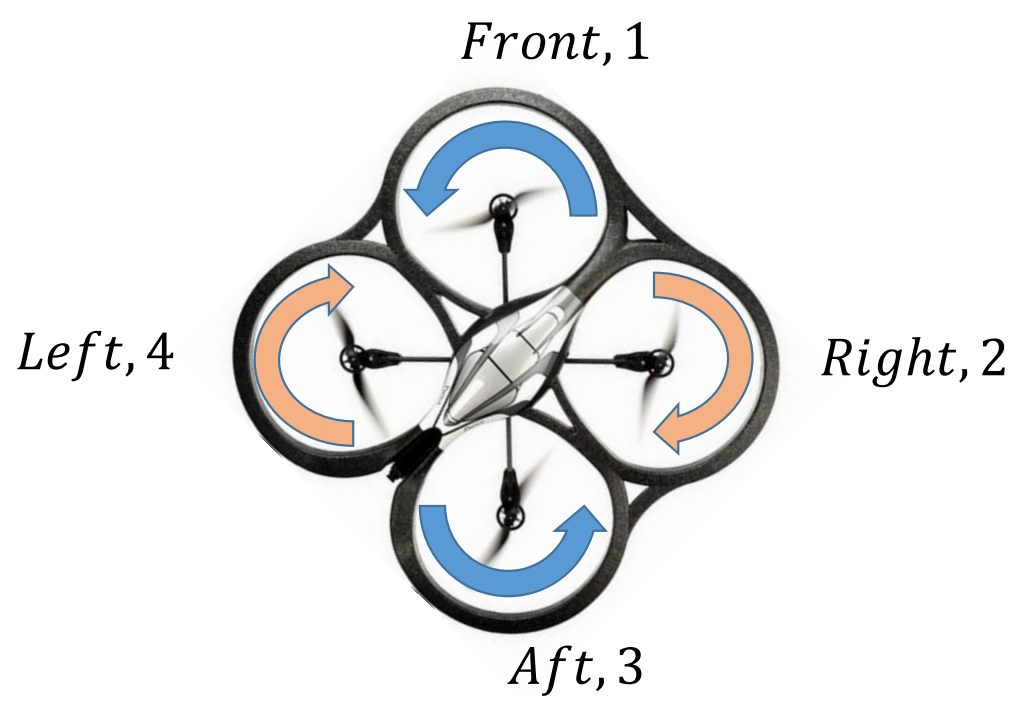

Figure 2.2: Rotor Enumeration

of thrust vectors from the center of mass and the thrust vectors.

$$
\vec{M}=\sum_{i} r_{i} \times F_{i}
$$

For the enumeration of rotor pairs, Figure 2.2 can be visited.

$$
\begin{aligned}
& \vec{M}=\left[\begin{array}{lll}
\vec{M}_{x} & \vec{M}_{y} & \vec{M}_{z}
\end{array}\right]^{T} \\
& \overrightarrow{r_{1}}=\left[l_{\text {arm }}, 0,0\right]^{T} \\
& \overrightarrow{r_{2}}=\left[0, l_{\text {arm }}, 0\right]^{T} \\
& \overrightarrow{r_{3}}=\left[-l_{\text {arm }}, 0,0\right]^{T} \\
& \overrightarrow{r_{4}}=\left[0,-l_{\text {arm }}, 0\right]^{T} \\
& {\left[M_{x}, M_{y}, M_{z}\right]^{T}=\left[\begin{array}{llll}
r_{1} \times e_{1} & r_{2} \times e_{2} & r_{3} \times e_{3} & r_{4} \times e_{4}
\end{array}\right]\left[F_{1}, F_{2}, F_{3}, F_{4}\right]^{T}}
\end{aligned}
$$

where

$$
\vec{F}_{i}=\left[0,0,-F_{i}\right] \forall i
$$

Then,

$$
\begin{aligned}
& M_{x}=l_{\text {arm }}\left(F_{4}-F_{2}\right) \\
& M_{y}=l_{\text {arm }}\left(F_{1}-F_{3}\right)
\end{aligned}
$$




\subsubsection{The Influence of Propeller Torque on Body Center of Mass}

The static relation between propeller and motor dynamics imposes that the force equation is related to the square root of rotation speed and propeller type, and thrust is linearly related to torque. The propeller dynamics imposes that the force generation due to the revolution of a propeller is related to the squared propeller angular speed as shown in Eqn. 2.7][130]:

$$
\begin{gathered}
F / F_{0}=\left(n / n_{0}\right)^{2} \\
F=\left(F_{0} / n_{0}^{2}\right) n^{2} \\
F=K_{n} n^{2}
\end{gathered}
$$

The next step is to define the relationship between the force and torque generated by propellers in order to indicate the influence of the rotation of propellers on the moment experienced in the z-direction of body frame. The equations of thrust and torque coefficients expressed for propellers in [131] can be safely used to deduce the relationship between torque and thrust.

$$
\begin{aligned}
& K_{T}=T_{P}\left(4 \pi^{2} / \rho n^{2} D^{4}\right) \\
& K_{Q}=Q_{P}\left(4 \pi^{2} / \rho n^{2} D^{5}\right)
\end{aligned}
$$

First, let us collect thrust and torque terms in one-side:

$$
\begin{aligned}
& T_{P}=K_{T}\left(\rho n^{2} D^{4} / 4 \pi^{2}\right) \\
& Q_{P}=K_{Q}\left(\rho n^{2} D^{5} / 4 \pi^{2}\right)
\end{aligned}
$$

By dividing one of these coefficients by the other one, a new coefficient expressing the linear relationship between the propeller torque and force is attained as can be seen in Eqn. 2.14 .

$$
\begin{gathered}
k_{m}=Q_{P} / T_{P}=\left(K_{Q} / K_{T}\right) D \\
M_{\text {prop }}=k_{m} F
\end{gathered}
$$

Notice that for quadrocopter, the propeller torques show their act on the body in $z_{B}$ direction; therefore, by adding the effect of each rotor, Eqn. 2.15 is now available to express the last component of the moments exerted on body as follows:

$$
M_{z}=k_{m}\left(-F_{1}+F_{2}-F_{3}+F_{4}\right)
$$




\subsubsection{Total Moment Exerted on Body Center of Mass}

Combining above derivations, the matrix that relates the moments exerted on body center of mass and the propeller force vectors can be constructed.

$$
\left[\begin{array}{l}
M_{x} \\
M_{y} \\
M_{z}
\end{array}\right]=\left[\begin{array}{c}
L \\
M \\
N
\end{array}\right]=\left[\begin{array}{cccc}
0 & -r & 0 & r \\
r & 0 & -r & 0 \\
-k_{m} & k_{m} & -k_{m} & k_{m}
\end{array}\right]\left[\begin{array}{c}
F_{1} \\
F_{2} \\
F_{3} \\
F_{4}
\end{array}\right]
$$

This matrix can further be extended with the total thrust produced assuming that no orientation of the vector pointing out from the nose of each motor is present in a direction other than $z_{B}$.

$$
\left[\begin{array}{c}
M_{x} \\
M_{y} \\
M_{z} \\
T_{c}
\end{array}\right]=\left[\begin{array}{c}
L \\
M \\
N \\
T_{c}
\end{array}\right]=\left[\begin{array}{cccc}
0 & -l_{\text {arm }} & 0 & l_{\text {arm }} \\
l_{\text {arm }} & 0 & -l_{\text {arm }} & 0 \\
-k_{m} & k_{m} & -k_{m} & k_{m} \\
1 & 1 & 1 & 1
\end{array}\right]\left[\begin{array}{c}
F_{1} \\
F_{2} \\
F_{3} \\
F_{4}
\end{array}\right]
$$

\section{$2.3 \quad$ Rigid Body Dynamics}

The rigid body equations of attitude motion can generally be described by the Newton-Euler equations of motion, or the energy-oriented approaches such as the Lagrange formulation. These can be expressed in the body frame or in the inertial frame, and can have different model structures and parametrizations. For instance, if the researcher is to deal with the attitude control, the initial point to be aware of is that the generated moments give rise to angular accelerations described by angular momentum dynamics. Eventually, the realization of angular acceleration changes in the model acts as inputs to the orientation dynamics and causes angular attitude changes.

\subsubsection{Rotation Matrix}

The orientation and position of the quadrotor cannot be described with respect to the moving body frame. Therefore, it is essential to define these properties of 
the body with respect to a fixed frame such as the Earth frame. The construction of such relations can be achieved by three rotations from Earth fixed frame to body fixed frame. To model this rotation, a specific sequence of Euler angles known as 3-2-1 (z-y-x) Euler angles can be utilized. 11 The first rotation around $g$ is from $s$ to $s$ through the yaw angle, $\psi$. Similarly, the second rotation is around 0 from $s$ to $s$ through the pitch angle, $\theta$ while the final rotation is around 0 from $s$ to $s$ through the roll angle, $\phi$. The rotations around each axis, $x, y$ and $z$ can respectively be represented with the following rotation matrices considering right-hand rule:

$$
\begin{aligned}
R(x, \phi) & =\left[\begin{array}{ccc}
1 & 0 & 0 \\
0 & \cos (\phi) & -\sin (\phi) \\
0 & \sin (\phi) & \cos (\phi)
\end{array}\right] \\
R(y, \theta) & =\left[\begin{array}{ccc}
\cos (\theta) & 0 & \sin (\theta) \\
0 & 1 & 0 \\
-\sin (\theta) & 0 & \cos (\theta)
\end{array}\right] \\
R(z, \psi) & =\left[\begin{array}{ccc}
\cos (\psi) & -\sin (\psi) & 0 \\
\sin (\psi) & \cos (\psi) & 0 \\
0 & 0 & 1
\end{array}\right]
\end{aligned}
$$

In order to find the rotation matrix from Earth-fixed to body frame, the matrices for each rotation can be multiplied.

$$
R(\phi, \theta, \psi)=R(x, \phi) R(y, \theta) R(z, \psi)
$$

Representing cosines by c and sines by s, the final form of the transformation matrix, also known as the direction cosine matrix, is as follows:

$$
R=\left[\begin{array}{ccc}
c(\theta) c(\psi) & s(\theta) c(\psi) s(\phi)-s(\psi) c(\phi) & c(\phi) s(\theta) c(\psi)+s(\psi) s(\phi) \\
s(\psi) c(\theta) & s(\psi) s(\theta) s(\phi)+c(\psi) c(\phi) & s(\psi) s(\theta) c(\phi)-s(\phi) c(\psi) \\
-s(\theta) & c(\theta) s(\phi) & c(\theta) c(\phi)
\end{array}\right]
$$

Since the time rates of the orientation dynamics are different from the body angular rates, another transformation matrix from body angular rates $\left[\begin{array}{lll}p & q & r\end{array}\right]^{T}$

\footnotetext{
1 Actually, this rotation is a combination of Tait-Bryan angles and does not belong to the family of proper Euler angles. However, as indicated in [100], the adoption of this misuse by the aerospace engineering literature is obvious.
} 
to Euler rates $\left[\begin{array}{lll}\dot{\phi} & \dot{\theta} & \dot{\psi}\end{array}\right]^{T}$ is necessary and can be calculated as in the following equations:

$$
\left[\begin{array}{lll}
p & q & r
\end{array}\right]^{T}=R_{r}(\theta, \phi)\left[\begin{array}{lll}
\dot{\phi} & \dot{\theta} & \dot{\psi}
\end{array}\right]^{T}
$$

where

$$
R_{r}(\theta, \phi)=R(x, \phi) R(y, \theta)=\left[\begin{array}{ccc}
1 & 0 & -\sin (\theta) \\
0 & \cos (\phi) & -\sin (\phi) \cos (\theta) \\
0 & -\sin (\phi) & \cos (\phi) \cos (\theta)
\end{array}\right]
$$

Finally, the so-called attitude propagation equations are achieved as follows. Note that these equations connect the Euler rates to the body fixed angular rates:

$$
\begin{gathered}
{\left[\begin{array}{c}
p \\
q \\
r
\end{array}\right]=\left[\begin{array}{ccc}
1 & 0 & -\sin (\theta) \\
0 & \cos (\phi) & -\sin (\phi) \cos (\theta) \\
0 & -\sin (\phi) & \cos (\phi) \cos (\theta)
\end{array}\right]\left[\begin{array}{c}
\dot{\phi} \\
\dot{\theta} \\
\dot{\psi}
\end{array}\right]} \\
{\left[\begin{array}{c}
\dot{\phi} \\
\dot{\theta} \\
\dot{\psi}
\end{array}\right]=\left[\begin{array}{ccc}
1 & \sin (\phi) \tan (\theta) & \tan (\theta) \cos (\phi) \\
0 & \cos (\phi) & -\sin (\phi) \\
0 & \sin (\phi) / \cos (\theta) & \cos (\phi) / \cos (\theta)
\end{array}\right]\left[\begin{array}{l}
p \\
q \\
r
\end{array}\right]}
\end{gathered}
$$

\subsubsection{Rate of Attitude Propagation Equations}

The attitude propagation equations for aerial vehicles as in 132 can safely be used for quadrotors to attain the relationship between Euler angles and body fixed rotational accelerations. The derivation of Eqn. 2.27 constructs this correspondence. Written in a shorter form:

$$
\left[\begin{array}{lll}
\dot{\phi} & \dot{\theta} & \dot{\psi}
\end{array}\right]^{T}=P(\phi, \theta)\left[\begin{array}{lll}
p & q & r
\end{array}\right]^{T}
$$

Then, deriving both sides,

$$
\frac{d}{d t}\left[\begin{array}{c}
\dot{\phi} \\
\dot{\theta} \\
\dot{\psi}
\end{array}\right]=\frac{d P(\phi, \theta)}{d t}\left[\begin{array}{l}
p \\
q \\
r
\end{array}\right]+P(\phi, \theta) \frac{d}{d t}\left[\begin{array}{l}
p \\
q \\
r
\end{array}\right]
$$

Expanding $d P(\phi, \theta) / d t$ as follows and then plugging into Eqn 2.29 yields:

$$
\frac{d P(\phi, \theta)}{d t}=\frac{d P(\phi, \theta)}{d \phi} \frac{d \phi}{d t}+\frac{d P(\phi, \theta)}{d \theta} \frac{d \theta}{d t}=\frac{d P(\phi, \theta)}{d \phi} \dot{\phi}+\frac{d P(\phi, \theta)}{d \theta} \dot{\theta}
$$




$$
\left[\begin{array}{l}
\ddot{\phi} \\
\ddot{\theta} \\
\ddot{\psi}
\end{array}\right]=P(\theta, \phi)\left[\begin{array}{l}
\dot{p} \\
\dot{q} \\
\dot{r}
\end{array}\right]+\frac{d P(\theta, \phi)}{d \theta}\left[\begin{array}{l}
p \\
q \\
r
\end{array}\right] \dot{\theta}+\frac{d P(\theta, \phi)}{d \phi}\left[\begin{array}{l}
p \\
q \\
r
\end{array}\right] \dot{\phi}
$$

Finally, plugging Eqn.2.26 into Eqn.2.31:

$$
\left[\begin{array}{c}
\ddot{\phi} \\
\ddot{\theta} \\
\ddot{\psi}
\end{array}\right]=P(\theta, \phi)\left[\begin{array}{c}
\dot{p} \\
\dot{q} \\
\dot{r}
\end{array}\right]+\frac{d P(\theta, \phi)}{d \theta} R_{r}(\theta, \phi)\left[\begin{array}{c}
\dot{\phi} \\
\dot{\theta} \\
\dot{\psi}
\end{array}\right] \dot{\theta}+\frac{d P(\theta, \phi)}{d \phi} R_{r}(\theta, \phi)\left[\begin{array}{c}
\dot{\phi} \\
\dot{\theta} \\
\dot{\psi}
\end{array}\right] \dot{\phi}
$$

where

$$
\frac{d P(\theta, \phi)}{d \theta} R_{r}(\theta, \phi)\left[\begin{array}{c}
\dot{\phi} \\
\dot{\theta} \\
\dot{\psi}
\end{array}\right] \dot{\theta}=\left[\begin{array}{c}
\dot{\theta} \dot{\psi} / \cos (\theta) \\
0 \\
\dot{\theta} \dot{\psi} \tan (\theta)
\end{array}\right]
$$

and

$$
\frac{d P(\theta, \phi)}{d \phi} R_{r}(\theta, \phi)\left[\begin{array}{c}
\dot{\phi} \\
\dot{\theta} \\
\dot{\psi}
\end{array}\right] \dot{\phi}=\left[\begin{array}{c}
\dot{\theta} \dot{\phi} \tan (\theta) \\
-\dot{\phi} \dot{\psi} \cos (\theta) \\
\dot{\theta} \dot{\phi} / \cos (\theta)
\end{array}\right]
$$

Combining all and shortening sines, cosines, and tangents, as s, c, and t:

$$
\left[\begin{array}{c}
\ddot{\phi} \\
\ddot{\theta} \\
\ddot{\psi}
\end{array}\right]=\left[\begin{array}{ccc}
1 & s(\phi) t(\theta) & t(\theta) c(\phi) \\
0 & c(\phi) & -s(\phi) \\
0 & s(\phi) / c(\theta) & c(\phi) / c(\theta)
\end{array}\right]\left[\begin{array}{c}
\dot{p} \\
\dot{q} \\
\dot{r}
\end{array}\right]+\left[\begin{array}{c}
\dot{\theta} \dot{\phi} t(\theta)+\dot{\theta} \dot{\psi} / c(\theta) \\
-\dot{\phi} \dot{\psi} c(\theta) \\
\dot{\theta} \dot{\phi} / c(\theta)+\dot{\theta} \dot{\psi} t(\theta)
\end{array}\right]
$$

\subsubsection{Angular Momentum Dynamics}

Note that since the effect of motor commands do not appear directly on the attitude angles, the angular momentum dynamics should be investigated to relate both elements through the rotational body-rates. In [132, it is described that for the element of mass, the moments on a body can be represented by the equation:

$$
\delta \vec{M}=\frac{d}{d t} \delta \vec{H}=\frac{d}{d t}(\vec{r} \times \vec{v}) \delta m
$$

Then, the angular momentum can be written as:

$$
\vec{H}=\sum \delta \vec{H}=\sum(\vec{r} \times \vec{v}) \delta m+\sum[\vec{r} \times(\vec{\omega} \times \vec{r})] \delta m
$$


where $\omega$ is the angular velocity of the vehicle and $\vec{r}$ is the position of the mass element from the center of mass. Besides, $\vec{H}$ can be written as:

$$
\vec{H}=\left[\begin{array}{ccc}
I_{x} & -I_{x y} & -I_{x z} \\
-I_{x y} & I_{y} & -I_{y z} \\
-I_{x z} & -I_{y z} & +I_{z}
\end{array}\right]\left[\begin{array}{l}
p \\
q \\
r
\end{array}\right]
$$

Here, $I_{x}, I_{y}, I_{z}$ are the mass moments of inertia of the vehicle body around indicated axes and $I_{x y}, I_{y z}, I_{x z}$ are the products of inertia. The derivative of the angular momentum referring to a rotating body frame can be represented by the following vector identity which brings out the angular momentum equations

$$
\vec{M}=I \vec{\omega}=\left.\frac{d \vec{H}}{d t}\right|_{B F F}+\vec{\omega} \times \vec{H}
$$

Assuming no change of inertia with time and inserting Eqn. 2.38,

$$
\begin{gathered}
\vec{M}=I \dot{\vec{\omega}}+\vec{\omega} \times I \vec{\omega} \\
\dot{\vec{\omega}}=I^{-1}[\vec{M}-\vec{\omega} \times I \vec{\omega}]
\end{gathered}
$$

Due to the symmetric structure of quadrocopter body, the products of inertia can be neglected. This fact results in the following statements for the rotational body rates:

$$
\begin{aligned}
& \dot{p}=\frac{M_{x}}{I_{x}}-q r \frac{\left(I_{z}-I_{y}\right)}{I_{x}} \\
& \dot{q}=\frac{M_{y}}{I_{y}}-r p \frac{\left(I_{x}-I_{z}\right)}{I_{y}} \\
& \dot{r}=\frac{M_{z}}{I_{z}}-p q \frac{\left(I_{y}-I_{x}\right)}{I_{z}}
\end{aligned}
$$

\subsection{Other Considerations for Modelling the Dynamics}

To advance the fidelity of modelling, several other segments should be mentioned. The rigid body model can be extended with simplified rotor dynamics and aerodynamics, using a combination of momentum and blade element theory for more accuracy. The produced aerodynamic forces and torques depend on 
operating conditions and vehicle motion. Furthermore, the research for smallscale UAVs makes it necessary to model the actuator dynamics. For now, this thesis leaves such considerations unattended.

\subsection{State-Space Representation of Attitude Dynamics}

The following steps are essential in defining state space representation of the above model dynamics. Firstly, because of the necessity to designate the indirect relationship between the attitude dynamics and the motor commands, the body rotational accelerations, $\dot{\overrightarrow{\omega_{B}}}$ can be defined as new control inputs, $\vec{v}$. Now assuming the states $\left[\begin{array}{llllll}\phi & \dot{\phi} & \theta & \dot{\theta} & \psi & \dot{\psi}\end{array}\right]^{T}$ for attitude dynamics, the system outputs can be chosen as the Euler angles. Combining this knowledge, the following equations can be written:

$$
\begin{aligned}
& {\left[\begin{array}{llllll}
x_{1} & x_{2} & x_{3} & x_{4} & x_{5} & x_{6}
\end{array}\right]^{T}=\left[\begin{array}{llllll}
\phi & \dot{\phi} & \theta & \dot{\theta} & \psi & \dot{\psi}
\end{array}\right]^{T}} \\
& {\left[\begin{array}{lll}
v_{1} & v_{2} & v_{3}
\end{array}\right]^{T}=\left[\begin{array}{lll}
\dot{p} & \dot{q} & \dot{r}
\end{array}\right]_{\text {command }}^{T}} \\
& \dot{x_{1}}=\dot{\phi}=x_{2} \\
& \dot{x_{2}}=\dot{\theta} \dot{\phi} t(\theta)+\frac{\dot{\theta} \dot{\psi}}{c(\theta)}+\dot{p}+s(\phi) t(\theta) \dot{q}+t(\theta) c(\phi) \dot{r} \\
& \dot{x_{2}}=x_{2} x_{4} t\left(x_{3}\right)+\frac{x_{4} x_{6}}{\cos \left(x_{3}\right)}+v_{1}+s\left(x_{1}\right) t\left(x_{3}\right) v_{2}+t\left(x_{3}\right) c\left(x_{1}\right) v_{3} \\
& \dot{x_{3}}=\dot{\theta}=x_{4} \\
& \dot{x_{4}}=-\dot{\phi} \dot{\psi} c(\theta)+c(\phi) \dot{q}-s(\phi) \dot{r}=-x_{2} x_{6} c\left(x_{3}\right)+c\left(x_{1}\right) v_{2}-s\left(x_{1}\right) v_{3} \\
& \dot{x_{5}}=\dot{\psi}=x_{6} \\
& \dot{x}_{6}=\frac{\dot{\theta} \dot{\phi}}{c(\theta)}+\dot{\theta} \dot{\psi} t(\theta)+\frac{s(\phi) \dot{q}}{c(\theta)}+\frac{c(\phi) \dot{r}}{c(\theta)} \\
& \dot{x_{6}}=\frac{x_{2} x_{4}}{c\left(x_{3}\right)}+x_{4} x_{6} t\left(x_{3}\right)+\frac{s\left(x_{1}\right) v_{2}}{c\left(x_{3}\right)}+\frac{c\left(x_{1}\right) v_{3}}{c\left(x_{3}\right)}
\end{aligned}
$$

Next, Euler angles can be collected in the matrix below:

$$
\left[\begin{array}{c}
\ddot{\phi} \\
\ddot{\theta} \\
\ddot{\psi}
\end{array}\right]=\left[\begin{array}{c}
x_{4}\left(x_{2} t\left(x_{3}\right)+x_{6} / c\left(x_{3}\right)\right) \\
-x_{2} x_{6} c\left(x_{3}\right) \\
x_{4}\left(x_{6} t\left(x_{3}\right)+x_{2} / c\left(x_{3}\right)\right)
\end{array}\right]+\left[\begin{array}{ccc}
1 & s\left(x_{1}\right) t\left(x_{3}\right) & t\left(x_{3}\right) c\left(x_{1}\right) \\
0 & c\left(x_{1}\right) & -s\left(x_{1}\right) \\
0 & s\left(x_{1}\right) / c\left(x_{3}\right) & c\left(x_{1}\right) / c\left(x_{3}\right)
\end{array}\right]\left[\begin{array}{l}
v_{1} \\
v_{2} \\
v_{3}
\end{array}\right]
$$




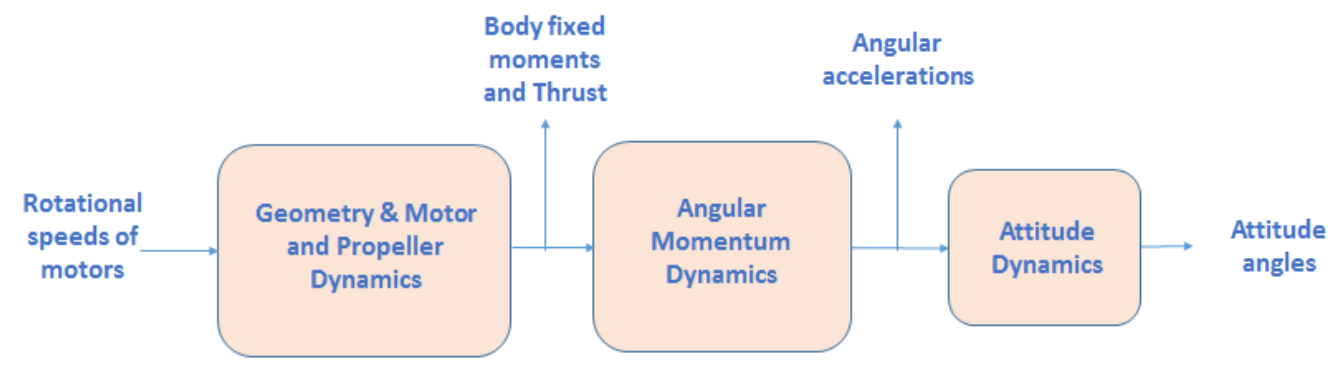

Figure 2.3: Modelled Dynamics Blocks for Quadrotor

Finally, the output dynamics can be summarized by:

$$
\ddot{\vec{y}}=f(\vec{x})+g(\vec{x}) \vec{v}
$$

The relations and parameters inside and between modelling blocks can be investigated in Figure 2.3 [133]. 


\section{CHAPTER 3}

\section{CONTROL STRATEGIES}

The main scope of this thesis is the discussion of various controller possibilities that may be applied to the orientation dynamics of quadrotor. This section provides the fundamental knowledge to be proficient in these methodologies.

\subsection{Non-Linear Dynamic Inversion}

To use the best aspects of the linear and non-linear approaches, designing attitude controller by non-linear dynamic inversion is an option available for selection. This section includes the basic principles for the non-linear dynamic inversion method firstly theorized and presented by Holzapfel in [134. The nonlinear dynamic inversion method can indeed be regarded as a tool to control a non-linear system as if it is linear.

\subsubsection{Regarded Dynamic System}

Consider a non-linear dynamic multi-variable system in the following form

$$
\begin{aligned}
& \dot{x}_{n \times 1}=f_{n \times 1}\left(x_{n \times 1}\right)+G_{n \times m}\left(x_{n \times 1}\right) \cdot u_{m \times 1} \\
& y_{m \times 1}=h_{m \times 1}\left(x_{n \times 1}\right)
\end{aligned}
$$


where $x, y$ and $u$ are respectively the state vector, the output vector and the control (input) vector:

$$
\begin{aligned}
x_{n \times 1} & =\left[\begin{array}{lll}
x_{1} & \ldots & x_{n}
\end{array}\right]^{T} \\
u_{m \times 1} & =\left[\begin{array}{lll}
u_{1} & \ldots & u_{m}
\end{array}\right]^{T} \\
y_{m \times 1} & =\left[\begin{array}{lll}
y_{1} & \ldots & y_{m}
\end{array}\right]^{T}
\end{aligned}
$$

Also note that $f(x), G(x)$ and $h(x)$ can be represented as follows. The vector fields $f, h$ and $g_{i}$ consist of non-linear functions of the state vector $x$. Moreover, these fields are sufficiently smooth, that is, continuously differentiable several times in their ranges $D_{x} \subset R^{n}$ and $D_{u} \subset R^{m}$.

$$
\begin{aligned}
f(x) & =\left[\begin{array}{lll}
f_{1}(x) & \ldots & f_{n}(x)
\end{array}\right]^{T} & \mid f: D_{x} \rightarrow R^{n} \\
h(x) & =\left[\begin{array}{lll}
h_{1}(x) & \ldots & h_{m}(x)
\end{array}\right]^{T} & \mid h: D_{x} \rightarrow R^{m} \\
G(x) & =\left[\begin{array}{lll}
g_{1} & \ldots & g_{m}
\end{array}\right]^{T} & \mid G: D_{u} \rightarrow R^{m} \\
g_{i}(x) & =\left[\begin{array}{lll}
g_{i 1}(x) & \ldots & g_{i n}(x)
\end{array}\right]^{T} & \mid 1 \leq i \leq m
\end{aligned}
$$

The system for the dynamics explained in the previous section possesses the same number of inputs as outputs. Such systems that are linear in controls are named "input affine". The modification of this method for the treatment of other systems with different numbers of inputs and outputs is also available.

\subsubsection{Dynamic Inversion}

Also called Input/Output linearization, the method of dynamic inversion aims at finding a non-linear state transformation $z=\Phi(x)$ so that the transformed system has a linear input/output behaviour. This kind of linearization is different from the general approaches used for linear control due to the fact that no approximation is required in the procedure. If such a transformation can be found, linear controller concepts can be exploited for the resulting linear system. Then, this controller can accomplish tasks like the stabilization of the system or the tracking of the prescribed reference trajectories [135], [136], [137]. 


\subsubsection{The Concept of Relative Degree}

Before finding the actual non-linear state feedback, some attention should be paid on the topic of the relative degree. The existence of a relative degree vector is necessary for the existence of a linearizing state feedback for MIMO systems. Similar to the MIMO system in the previous section, a SISO system such as $\dot{x}=f(x)+g(x) u$ and $y=h(x)$ with a scalar input $u_{i}$, a single output $y_{i}$, a single vector field $g_{i}(x)$ and a scalar function, $h(x)$ can be considered at this point. Deriving the output variable with respect to time, it is clear that:

$$
\dot{y}=\frac{d y}{d t}=\frac{\partial y}{\partial x} \cdot \frac{\partial x}{\partial t}=\frac{\partial h}{\partial x} \cdot \frac{\partial x}{\partial t}=\frac{\partial h}{\partial x} \cdot f(x)+\frac{\partial h}{\partial x} \cdot g(x) \cdot u=L_{f} h(x)+L_{g} h(x) \cdot u
$$

With the assumption that the input $u$ in a given point $x_{0}$ has no influence on the first derivative of $\mathrm{y}$, in other words $L_{g} h\left(x_{0}\right)=0$, then the expression can be simplified to

$$
\dot{y}=L_{f} h(x)
$$

The derivation should be proceeded until the input appears explicitly in the $r^{\text {th }}$ time derivative of the output which is then actually the lowest derivative of $y$ directly influenced by the control variable. This means that the control inputs have no direct influence on all minor derivatives, $y^{i}(t)$ with $i \leq r$. The significance behind this fact is that the degree up to which the output variable should be derived can be set directly using the available control variable. This number $r$ is called the relative degree of the SISO system of the subject. This degree is also a measure of the least possible delay responsive to the system output at $x_{0}$ on the control variable.

\subsubsection{The Relative Degree of Multi-Variable Systems}

The relative degree of a multi-variable system can be attained as follows. Let $\lambda$ a real-valued scalar and smooth function such that $\lambda: U \rightarrow R$ and let $f(x)$ a vector field such that $f: U \rightarrow R^{n}$, both defined on an open subset $U \in R^{n}$. The Lie derivative, defined as the gradient of $\lambda(x)$ over $f(x)$, is then:

$$
L_{f} \lambda(x)=\frac{\partial \lambda(x)}{\partial x} \cdot f(x)=\left[\begin{array}{lll}
\frac{\partial \lambda}{\partial x_{1}} & \cdots & \frac{\partial \lambda}{\partial x_{n}}
\end{array}\right] \cdot f(x)
$$


The repeated application of the Lie derivative with respect to the same vector field is defined recursively according to:

$$
\begin{aligned}
& L_{f}^{k} \lambda(x)=\frac{\partial\left(L_{f}^{k-1} \lambda(x)\right)}{\partial x} \cdot f(x) \\
& L_{f}^{0} \lambda(x)=\lambda(x)
\end{aligned}
$$

Letting $g(x)$ another vector field with $g: U \rightarrow R^{n}$, successive application of the Lie derivative with respect to $f$ and $g$ are attained as:

$$
L_{g} L_{f} \lambda(x)=\frac{\partial\left(L_{f} \lambda(x)\right)}{\partial x} \cdot g(x)
$$

Finally, the application of Lie-derivative on a matrix $G(x)=\left[\begin{array}{lll}g_{1}(x) & \ldots & g_{m}(x)\end{array}\right]$ such that $g_{i}(x): U \rightarrow R^{n}$. Then, the related lie derivative is:

$$
L_{G} \lambda(x)=\left[\begin{array}{lll}
L_{g_{1}} \lambda(x) & \ldots & L_{g_{m}} \lambda(x)
\end{array}\right]
$$

Since the basic knowledge on Lie-derivatives is constructed, the successive derivation of each system output leads to the determination of the relative degree of the MIMO system:

$$
\begin{aligned}
\dot{y}_{i} & =\frac{d y_{i}}{d t}=\frac{\partial y_{i}}{\partial x} \cdot \frac{\partial x}{\partial t} \\
& =\frac{\partial h_{i}}{\partial x} \cdot \frac{\partial x}{\partial t}=\frac{\partial h_{i}}{\partial x} \cdot f(x)+\frac{\partial h_{i}}{\partial x} \cdot G(x) \cdot u \\
& =L_{f} h_{i}(x)+L_{G} h_{i}(x) \cdot u=L_{f} h_{i}(x) \\
\ddot{y}_{i} & =\frac{d \dot{y}_{i}}{d t}=\frac{\partial \dot{y}_{i}}{\partial x} \cdot \frac{\partial x}{\partial t}=\frac{\partial L_{f} h_{i}}{\partial x} \cdot \frac{\partial x}{\partial t} \\
& =\frac{\partial L_{f} h_{i}}{\partial x} \cdot f(x)+\frac{\partial L_{f} h_{i}}{\partial x} \cdot G(x) \cdot u \\
& =L_{f}^{2} h_{i}(x)+L_{G} L_{f} h_{i}(x) \cdot u=L_{f}^{2} h_{i}(x)
\end{aligned}
$$

Assume the direct influence is observed at $r^{\text {th }}$ differentiation. Note that up to now there is no direct influence of control on the output.

$$
\begin{aligned}
y_{i}^{r_{i}} & =\frac{d y_{i}^{r_{i}-1}}{d t}=\frac{\partial y_{i}^{r_{i}-1}}{\partial x} \cdot \frac{\partial x}{\partial t}=\frac{\partial L_{f}^{r_{i}-1} h_{i}}{\partial x} \cdot \frac{\partial x}{\partial t} \\
& =\frac{\partial L_{f}^{r_{i}-1} h_{i}}{\partial x} \cdot f(x)+\frac{\partial L_{f}^{r_{i}-1} h_{i}}{\partial x} \cdot G(x) \cdot u \\
& =L_{f}^{r_{i}} h_{i}(x)+L_{G} L_{f}^{r_{i}-1} h_{i}(x) \cdot u
\end{aligned}
$$


A multi-variable system has a vector of relative degree $\left[r_{1}, \ldots, r_{m}\right]$ at a point $x_{0} \in$ $R^{n}$. Remembering the facts $L_{g_{j}} L_{f}^{k} h_{i}(x)=0 \forall 1 \leq j \leq m, k \leq r_{i}-1,1 \leq i \leq m$ when $x$ is in the neighbourhood of $x_{0}$, the relative degree of the whole system is equal to the summation of the relative degree of each output as indicated in Eqn 3.13 ,

$$
r=\sum_{i=1}^{m} r_{i} \leq n
$$

Expanding Eqn. $3.12 \mathrm{c}$ which is valid $\forall i$ such that $1 \leq i \leq m$,

$$
y_{i}^{r_{i}}=L_{f}^{r_{i}} h_{i}(x)+\sum_{k=1}^{m} L_{g_{k}} L_{f}^{r_{i}-1} h_{i}(x) \cdot u_{k}
$$

The dynamic system can finally be written as:

$$
\left[\begin{array}{c}
y_{1}^{r_{1}} \\
y_{2}^{r_{2}} \\
\vdots \\
y_{m}^{r_{m}}
\end{array}\right]=\left[\begin{array}{c}
L_{f}^{r_{1}} h_{1} \\
L_{f}^{r_{2}} h_{2} \\
\vdots \\
L_{f}^{r_{m}} h_{m}
\end{array}\right]+\left[\begin{array}{cccc}
L_{g_{1}} L_{f}^{r_{1}-1} h_{1} & L_{g_{2}} L_{f}^{r_{1}-1} h_{1} & \ldots & L_{g_{m}} L_{f}^{r_{1}-1} h_{1} \\
L_{g_{1}} L_{f}^{r_{2}-1} h_{2} & L_{g_{2}} L_{f}^{r_{2}-1} h_{2} & \ldots & L_{g_{m}} L_{f}^{r_{2}-1} h_{2} \\
\vdots & \vdots & \ddots & \vdots \\
L_{g_{1}} L_{f}^{r_{m}-1} h_{m} & L_{g_{2}} L_{f}^{r_{m}-1} h_{m} & \ldots & L_{g_{m}} L_{f}^{r_{m}-1} h_{m}
\end{array}\right]\left[\begin{array}{c}
u_{1} \\
u_{2} \\
\vdots \\
u_{m}
\end{array}\right]
$$

In a more compact form, this matrix is actually:

$$
y_{i}^{r_{i}}=b(x)+A(x) \cdot u
$$

In multi-variable systems, the non-singularity of the decoupling matrix, $A(x)$, is one of the defining characteristics of the relative degree and thus taken into consideration. The existence of a relative degree vector guarantees the invertibility of the matrix. If the matrix $A(x)$ is singular, that is the rank of $A$ is less than $m$, then the system has no well-defined relative degree.

The relative degree of the system can be found by investigating the equations in the previous chapter. No direct influence of the control input is present obviously from Eqn.s 2.45, 2.48 and 2.50. Each of the other equations, namely, 2.47, 2.49, and 2.52 has the relative degree of 2, summing up to a total of 6 . This means that the number of states and the relative degree of the system are equal resulting in the non-existence of the internal dynamics within the system. 


\subsubsection{The Transformation of the System Dynamics}

In order for the system to have the properties of a linear Input/Output dynamics, the transformation explained in this section is crucial. Making use of the fact that every output $y_{i}$ having a relative degree of $r_{i}$ is independent from the control input in the first $r_{i}-1$ derivatives, and therefore $L_{g} L_{f}^{k} h(x)=0$ as long as $k \leq r-1$, the following transformation are available for $1 \leq j \leq r_{k}$ when $1 \leq k \leq m$ :

$$
z_{j}=\Phi_{j}(x)=y_{k}^{j-1}=L_{f}^{j-1} h_{k}(x)
$$

Using this analogy, the following transformation can be achieved for the whole system,

$$
\left[\begin{array}{c}
z_{1} \\
z_{2} \\
\vdots \\
z_{r_{1}+\ldots+r_{m-1}+1} \\
\vdots \\
z_{r}
\end{array}\right]=\left[\begin{array}{c}
y_{1}^{0} \\
\vdots \\
y_{1}^{r_{1}-1} \\
\vdots \\
y_{m}^{0} \\
\vdots \\
y_{m}^{r_{m}-1}
\end{array}\right]=\left[\begin{array}{c}
L_{f}^{0} h_{1}(x) \\
\vdots \\
L_{f}^{r_{1}-1} h_{1}(x) \\
\vdots \\
L_{f}^{0} h_{m}(x) \\
\vdots \\
L_{f}^{r_{m}-1} h_{m}(x)
\end{array}\right]
$$

In summary, the transformation and its inverse are in the following form:

$$
z=\Phi(x) \rightarrow x=\Phi^{-1}(z)
$$

The discussion remaining at this point is the availability of a unique inversion for the transformation in Eqn. 3.19. If the system has full relative degree, the number of equations and the number of states are equal, resulting in a diffeomorphism. Otherwise, if the system is not full relative degree, additional equations as much as the number of the difference between the state outputs and the relative degree are required. In such a situation, the transformation has to be at least locally uniquely invertible. Note that diffeomorphism can be defined such that a bijective C-mapping $f: U \rightarrow V$ on open sets of $U \in X$ and $V \in Y$ are called diffeomorphism if the inverse mapping $f^{-1}: V \rightarrow U$ is also a C-mapping. Such a diffeomorphism is global if the mapping and its inverse are invertible $\forall x \in R^{n}$ and smooth (i.e. continuously differentiable). A 
local diffeomorphism can be referred to if these criteria are not met globally but locally around a point $x_{0}$.

\subsubsection{Linearization of State Feedback}

The state transformation explained so far paves the way for the construction of a non-linear state feedback which generates a linear and decoupled input-output behaviour. In order to apply the ideas for non-linear dynamic inversion, the control input in the following form can be selected:

$$
u=\alpha(x)+\beta(x) \nu
$$

At this point, new control variables symbolized with $\nu$ and named "pseudocontrols" should be defined for the transformed system: Now inserting Eqn. 3.20 into 3.16, $\alpha$ and $\beta$ can be determined:

$$
\left[y_{i}^{r_{i}}\right]=b(x)+A(x) \cdot u=b(x)+A(x) \cdot(\alpha(x)+\beta(x) \nu)
$$

Choosing $\alpha$ and $\beta$ as in Eqn. $3.22 \mathrm{~b}$ allows the reduction of the dynamics of the closed loop to pseudo controls as indicated in Eqn. 3.24 .

$$
\begin{aligned}
& \alpha(x)=A^{-1}(x) \cdot b(x) \\
& \beta(x)=A^{-1}(x)
\end{aligned}
$$

Therefore, the actual control values that will be fed into the track are:

$$
u=A^{-1}(x)[\nu-b(x)]
$$

The final form of the relation for the input/output dynamics is:

$$
\left[\begin{array}{lll}
y_{1}^{r_{1}} & \ldots & y_{m}^{r_{m}}
\end{array}\right]^{T}=\nu_{m \times 1}
$$

This means a replacement of the original, coupled and non-linear dynamics of the output system by pure integrator decoupled chains between the pseudo control variables and the associated output variables. The knowledge gained so far will be exploited to form a basis of the attitude control scheme explained within this thesis. 


\subsubsection{Non-Linear Dynamic Inversion of Attitude Dynamics of a Quadro- copter}

Bringing together the relations expressed in Eqn.s 2.53 and 2.44, the representation of the form 3.16 has been reached for output variables. Applying the pseudo control equation in Eqn. 3.24 by choosing outputs as Euler angles and remembering that the relative degree of each output is 2 (i.e. $r_{1}=r_{2}=r_{3}=2$ ), the following relationship is deduced.

$$
\left[\begin{array}{lll}
\nu_{1} & \nu_{2} & \nu_{3}
\end{array}\right]^{T}=\left[\begin{array}{lll}
\ddot{y_{1}} & \ddot{y_{2}} & \ddot{y_{3}}
\end{array}\right]^{T}=\left[\begin{array}{lll}
\ddot{\Phi} & \ddot{\Theta} & \ddot{\Psi}
\end{array}\right]^{T}
$$

Now, taking the inverse of Eqn. 2.53 and deriving the relationships for alternative control inputs, $v$,

$$
v=A^{-1}(x)[\nu-b(x)]
$$

can be attained where

$$
\begin{gathered}
A^{-1}(x)=\left[\begin{array}{ccc}
1 & 0 & -s\left(x_{3}\right) \\
0 & c\left(x_{1}\right) & s\left(x_{1}\right) c\left(x_{3}\right) \\
0 & -s\left(x_{1}\right) & c\left(x_{1}\right) c\left(x_{3}\right)
\end{array}\right] \\
b(x)=\left[\begin{array}{c}
x_{4}\left(x_{2} t\left(x_{3}\right)+x_{6} / c\left(x_{3}\right)\right) \\
-x_{2} x_{6} c\left(x_{3}\right) \\
x_{4}\left(x_{6} t\left(x_{3}\right)+x_{2} / c\left(x_{3}\right)\right)
\end{array}\right]
\end{gathered}
$$

Taking the inverse of Eqn. 2.35 as explained before and inserting pseudo- and alternative controls into Eqn. 3.29 .

$$
\left[\begin{array}{c}
v_{1} \\
v_{2} \\
v_{3}
\end{array}\right]=\left[\begin{array}{ccc}
1 & 0 & -s \theta \\
0 & c \phi & s \phi c \theta \\
0 & -s \phi & c \phi c \theta
\end{array}\right]\left[\begin{array}{c}
\nu_{1} \\
\nu_{2} \\
\nu_{3}
\end{array}\right]-\left[\begin{array}{c}
\frac{\dot{\theta}}{c \theta}(\dot{\phi} s \theta+\dot{\psi}) \\
-\dot{\phi} \dot{\psi} c \theta \\
\frac{\dot{\theta}}{c \theta}(\dot{\phi}+s \theta \dot{\psi})
\end{array}\right]
$$

$$
\left[\begin{array}{l}
v_{1} \\
v_{2} \\
v_{3}
\end{array}\right]=\left[\begin{array}{ccc}
1 & 0 & -s \theta \\
0 & c \phi & s \phi c \theta \\
0 & -s \phi & c \theta c \phi
\end{array}\right]\left[\begin{array}{c}
\nu_{1} \\
\nu_{2} \\
\nu_{3}
\end{array}\right]+\dot{\theta} \dot{\phi}\left[\begin{array}{c}
0 \\
-s \phi \\
-c \phi
\end{array}\right]+\dot{\psi} \dot{\phi}\left[\begin{array}{c}
0 \\
-c \phi c \theta \\
-s \phi c \theta
\end{array}\right]+\dot{\theta} \dot{\psi}\left[\begin{array}{c}
c \theta \\
-s \phi s \theta \\
-c \phi s \theta
\end{array}\right]
$$


Here, alternative and pseudo controls correspond to the body angular rates and Euler accelerations respectively. In order to complete the inversion of attitude dynamics, several additional inversions are required. Firstly, the inverse of angular momentum dynamics is given in Eqn. 2.40. Restating this equation:

$$
\vec{M}=I \dot{\vec{\omega}}+\vec{\omega} \times I \vec{\omega}
$$

More explicitly,

$$
\begin{aligned}
& M_{x}=I_{x} \dot{p}+q r\left(I_{z}-I_{y}\right) \\
& M_{y}=I_{y} \dot{q}+r p\left(I_{x}-I_{z}\right) \\
& M_{z}=I_{z} \dot{r}+p q\left(I_{y}-I_{x}\right)
\end{aligned}
$$

Secondly, the relationship between propeller forces and the produced body moments and total thrust should be inverted. The inverse of Eqn. 2.18 is

$$
\left[\begin{array}{l}
F_{1} \\
F_{2} \\
F_{3} \\
F_{4}
\end{array}\right]=\left[\begin{array}{cccc}
0 & -L & 0 & L \\
L & 0 & -L & 0 \\
-k_{m} & k_{m} & -k_{m} & k_{m} \\
1 & 1 & 1 & 1
\end{array}\right]^{-1}\left[\begin{array}{c}
M_{x} \\
M_{y} \\
M_{z} \\
T_{c}
\end{array}\right]=\left[\begin{array}{c}
L \\
M \\
N \\
T_{c}
\end{array}\right]
$$

Finally, the thrust forces found in Eqn. 3.33 are inputs to the inverse of Eqn. 3.34 which gives the rotational speeds of the brushless motors:

$$
\left[\begin{array}{llll}
n_{1} & n_{2} & n_{3} & n_{4}
\end{array}\right]^{T}=\left[\begin{array}{llll}
F_{1} / k_{m} & F_{2} / k_{m} & F_{3} / k_{m} & F_{4} / k_{m}
\end{array}\right]^{T}
$$

Figure 3.1 summarizes the input/output linearized non-linear dynamic quadrotor system and the use of state feedback in this procedure. The essence of the idea behind this is that the non-linear characteristics of the quadrocopter dynamics are not neglected during the inversion. As a result, the linearization holds for the whole range of flight.

\subsection{Reference Model and Error Dynamics}

The controller approaches that will be presented in the following chapters are based on a reference model. Therefore, this section aims at building a reference 


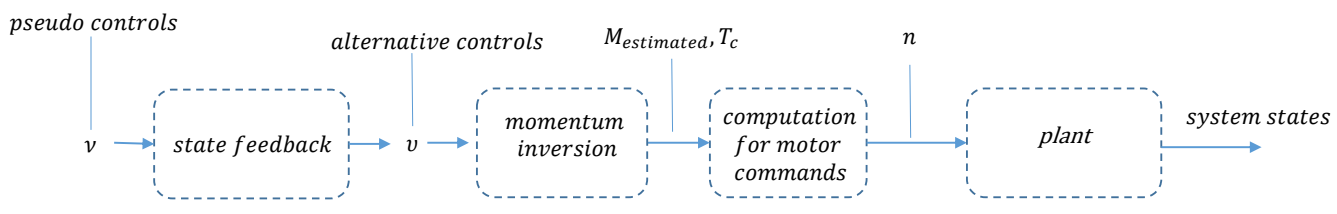

Figure 3.1: Scheme of State Feedback

model for the attitude dynamics of a quadrocopter system. In the previous chapter, the relative degree of each attitude output has been found as 2 . This fact points out that the pseudo-control inputs are necessarily the second derivatives of the control commands. The reason for implementing a reference model into this controller structure is that adequately "smooth" reference trajectories and their successive derivations can be generated by such a reference model, of second order for this case, so that the highest derivatives of the commands required for the scheme exist. A well-known linear second order reference model in the following form can be employed in this sense:

$$
\ddot{y}_{r e f}+2 \omega_{0} \zeta \dot{y}_{r e f}+\omega_{0}^{2} y_{r e f}=\omega_{0}^{2} y_{c m d}
$$

where $\omega_{0}$ is the natural frequency and $\zeta$ is the damping ratio. With some manipulations, the second derivative can be left in the left side of Eqn. 3.35 and inserting orientation angles into $x$ variable, a matrix as in Eqn. 3.37 can be attained.

$$
\ddot{y}_{r e f}=\omega_{0}^{2}\left(y_{c m d}-y_{r e f}\right)-2 \omega_{0} \zeta \dot{y}_{r e f}
$$

$$
\left[\begin{array}{l}
\ddot{\phi}_{r e f} \\
\ddot{\theta}_{r e f} \\
\ddot{\psi}_{\text {ref }}
\end{array}\right]=\left[\begin{array}{ccc}
\omega_{0, \phi}^{2} & 0 & 0 \\
0 & \omega_{0, \theta}^{2} & 0 \\
0 & 0 & \omega_{0, \psi}^{2}
\end{array}\right]\left[\begin{array}{l}
\phi_{c m d}-\phi_{\text {ref }} \\
\theta_{c m d}-\theta_{r e f} \\
\psi_{c m d}-\psi_{\text {ref }}
\end{array}\right]-\left[\begin{array}{ccc}
2 \omega_{0, \phi} \zeta_{\phi} & 0 & 0 \\
0 & 2 \omega_{0, \theta} \zeta_{\theta} & 0 \\
0 & 0 & 2 \omega_{0, \psi} \zeta_{\psi}
\end{array}\right]\left[\begin{array}{l}
\dot{\phi}_{r e f} \\
\dot{\theta}_{r e f} \\
\dot{\psi}_{r e f}
\end{array}\right]
$$

In model reference control schemes, the desired plant behaviour is described by a reference model and is driven by a reference input. The control law is then 
developed so that the modelling of the closed-loop of the system and that of the plant are equivalent to the reference model. Such a matching guarantees that the plant will behave like the reference model for any reference input signal.

Since the presence of any modelling error, external disturbance or uncertainty is quite probable, there occurs a difference between the second derivative of the system outputs and the pseudo controls provided by the second order reference model constructed for the attitude dynamics.

The integration of this second derivative twice yields to a deviation of the system outputs from the reference commands initially given. This is caused by the fact that the integrators bring forward two poles located at the origin of the state space; thus, the instability of the system. The solution to overcome this problem is taking the error dynamics into account and introducing an error controller by extending the reference pseudo controls with the terms of this controller. Since the system has already been decoupled using the method of state feedback linearization, the error dynamics are available for the description in single input single output form. Let define control error and its first and second time derivatives:

$$
\begin{aligned}
& e=y_{i, r e f}-y_{i} \\
& \dot{e}=\dot{y}_{i, r e f}-\dot{y}_{i} \\
& \ddot{e}=\ddot{y}_{i, r e f}-\ddot{y}_{i}
\end{aligned}
$$

The next step is to show the equation of the extended pseudo control:

$$
v_{i}=v_{i, r e f}+v_{\text {extend }}
$$

where

$$
\begin{aligned}
v_{i, r e f} & =\ddot{y}_{i, r e f} \\
v_{\text {extend }} & =k_{d} \dot{e}+k_{p} e+k_{i} e / s
\end{aligned}
$$

Note that the extended term actually makes use of a PID-like structure. Now, supposing that there exists some kind of an error in modelling or uncertainty in the system, the corresponding difference between the second derivative of the 
output and the pseudo control can be labelled as $\Delta_{i}(x)$.

$$
\begin{aligned}
\delta_{i}(x) & =\ddot{y}_{i}-v_{i} \\
v_{i} & =-\Delta_{i}(x)+\ddot{y}_{i}
\end{aligned}
$$

Now inserting Eqn.s 3.41a, 3.41b and 3.40b into Eqn. 3.39:

$$
\ddot{y}_{i}-\Delta_{i}(x)=\ddot{y}_{i, r e f}+k_{d} \dot{e}+k_{p} e+k_{i} e / s
$$

Arranging error terms in one side:

$$
\ddot{e}+k_{d} \dot{e}+k_{p} e+k_{i} e / s=-\Delta_{i}(x)
$$

In order to explicitly determine the error dynamics, let the vector of error states, $e_{v}=\left[\begin{array}{lll}\int e \cdot d t & e & \dot{e}\end{array}\right]^{T}$ exist and thus the error dynamics can be written as:

$$
\begin{gathered}
\dot{e_{v}}=A_{e d} \cdot e_{v}+B_{e d} \cdot \Delta \\
\dot{e_{v}}=\left[\begin{array}{rrr}
0 & 1 & 0 \\
0 & 0 & 1 \\
-k_{i} & -k_{p} & -k_{d}
\end{array}\right] \cdot e_{v}+\left[\begin{array}{c}
0 \\
0 \\
-1
\end{array}\right] \cdot \Delta
\end{gathered}
$$

After attaining the relation 3.45, the stability of the error dynamics can be explored by finding the eigenvalues of $A_{e d}$ :

$$
\begin{aligned}
\left|s \cdot I-A_{e d}\right| & =\left|\begin{array}{ccc}
s & -1 & 0 \\
0 & s & -1 \\
k_{i} & k p & s+k d
\end{array}\right| \\
& =s^{3}+k_{d} s^{2}+k_{p} s+k_{i}
\end{aligned}
$$

If Eqn. $3.46 \mathrm{~b}$ is assumed to be equal to the following $3^{\text {rd }}$ order lag element, the coefficients of $k_{p}$ and $k_{d}$ can be determined from the natural frequency and the damping ratio and $k_{i}$ can be chosen accordingly:

$$
\begin{gathered}
s\left(s^{2}+2 \omega_{0} \zeta s+\omega_{0}^{2}\right)+k_{i}=s^{3}+k_{d} s^{2}+k_{p} s+k_{i} \\
k_{d}=2 \omega_{0} \zeta \\
k_{p}=\omega_{0}^{2}
\end{gathered}
$$




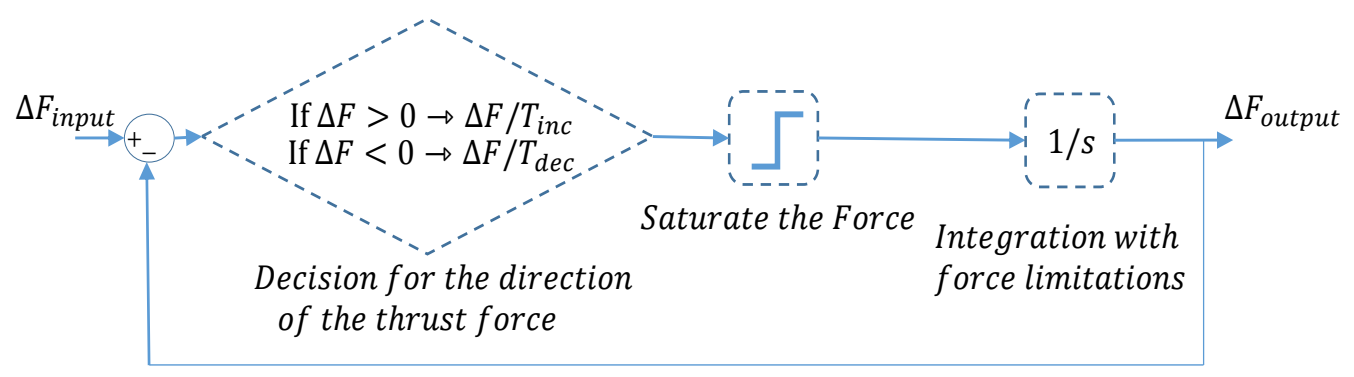

Figure 3.2: The Thrust Dynamics Model

\subsubsection{The Thrust Model of the Motor and Propeller Dynamics}

In the methodology of the dynamic modelling of the quadrocopter system, the motor and propeller dynamics is left out of the scope. Inside the reference model, however, the use of a first-order lag element which describes the thrust dynamics can be beneficial. Such a modelling which is also taken into consideration in this work is present in [133]. This thrust modelling, constructed by AscTech, the producer of Humming-bird quadrocopters, and the author of [133], includes different time constants for increases and decreases in input signals and imposes limitations on the first time derivative of these signal. Another significant point of this modelling is that the increase in thrust signal is related to the motor controller, the maximum power of the motor and the implemented internal slew rate while any decreasing signal is linked to the inertia of propeller and motor combination and the drag exerted on the propeller. Figure 3.2 explains this dynamic thrust model.

\subsubsection{Parameter Selection}

Not only the parameters inside the reference model such as the natural frequency and the damping ratio of each movement axis of the body, and the error controller gains need to be determined but the reference model should also be advanced with the addition of the thrust dynamics of the brushless motor and that the criteria for maximal conditions of body fixed angular accelerations. Note these criteria may change according to the characteristics of a flight phase. 
For example, if hover condition is the phase of interest, the required thrust force is equal to the weight of the model and the force per each motor is quarter of this force. Then, the maximum criteria can be obtained as in Eqn.s 3.50a, 3.50b and 3.50c;

$$
\begin{aligned}
& \dot{p}_{\text {max }}=\frac{\left|M_{x, \text { max }}\right|}{I_{x x}}=\frac{2 r\left|F_{\text {phase }}\right|}{I_{x x}} \\
& \dot{q}_{\text {max }}=\frac{\left|M_{y, \text { max }}\right|}{I_{y y}}=\frac{2 r\left|F_{\text {phase }}\right|}{I_{y y}} \\
& \dot{r}_{\text {max }}=\frac{\left|M_{z, \text { max }}\right|}{I_{z z}}=\frac{4 k_{m}\left|F_{\text {phase }}\right|}{I_{z z}}
\end{aligned}
$$

As a note, Eqn.s 3.50a and $3.50 \mathrm{~b}$ are equal to each other since the structure of a quadrocopter possesses the property of symmetry in $\mathrm{x}$ and $\mathrm{y}$ axes. In the formation of the reference model, the main reason for the interest in the maximal properties in body-fixed frame is the existence of actuator saturations. The sensor limit on the Hummingbird quadrocopter is given as $300 \mathrm{deg} / \mathrm{s}$. The rotational rates within the reference model are based on this limitation in each axis. To summarize, the reference model is augmented with the actuator dynamics and saturation limitations. Moreover, this augmentation contains the simple inversion to force input to the thrust model as well as the transformation backwards. In order to respond to the overshooting-related concerns, the damping ratio is selected as "1". Before determining the natural frequencies of attitude axes, various trials should be conducted for this critically damped system by the following simulations schemes. The simulation for the reference model is necessary due to the saturations in non-linear actuator and reference dynamics. The schemes of these simulations are shown in Figure 3.3. As can be examined in these schemes, pitch reference model includes the dynamics of the motors labelled as 1 and 3 that are directly in association with the pitch attitude change. Similarly, the roll reference model is based on motors 2 and 4 related to the roll attitude change while the yaw model contains the influence of all motors. Furthermore, the roll and pitch reference models are actually identical due to the structural symmetry and therefore regarded the same from this point on.

The selection of the natural frequencies for the reference models can be made by evaluating the performance of different options through the simulations already depicted. In the simulations, step inputs of $30 \mathrm{deg} / \mathrm{s}, 30 \mathrm{deg} / \mathrm{s}$ and 27 


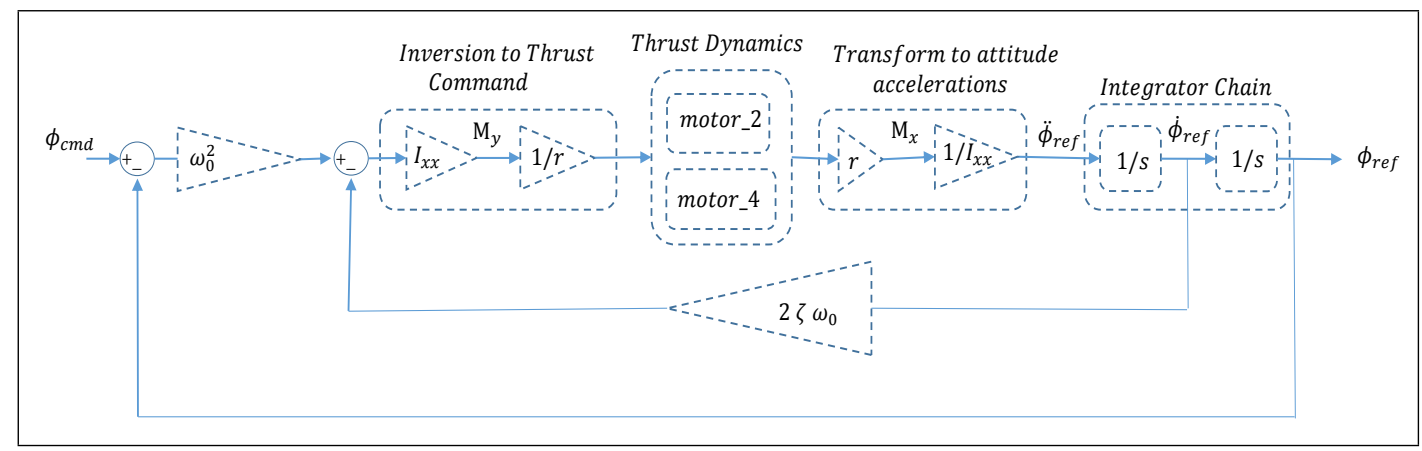

(a) Reference Model Structure for Roll

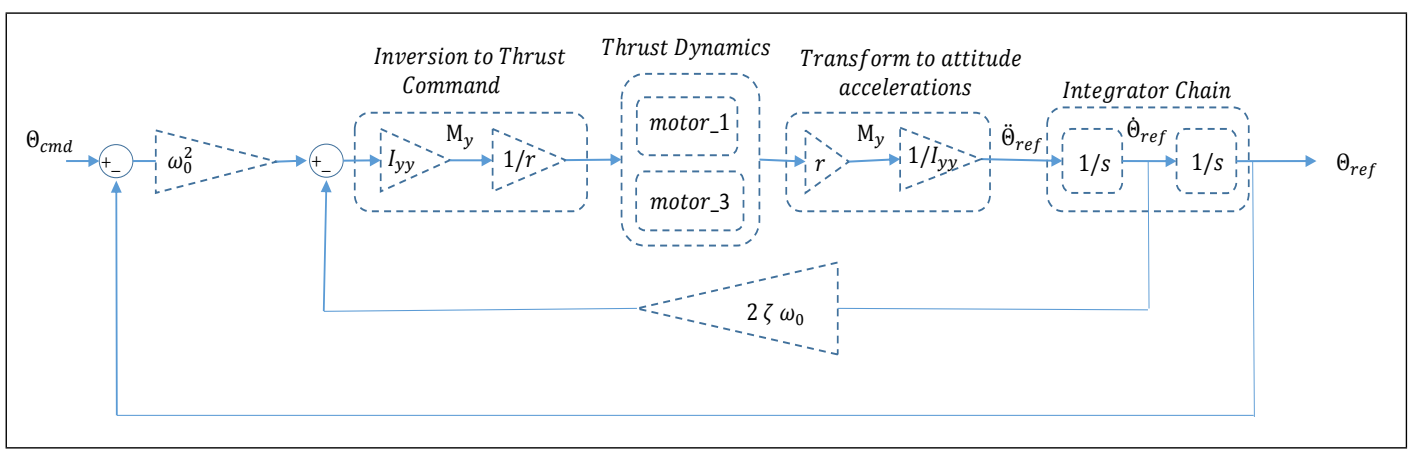

(b) Reference Model Structure for Pitch

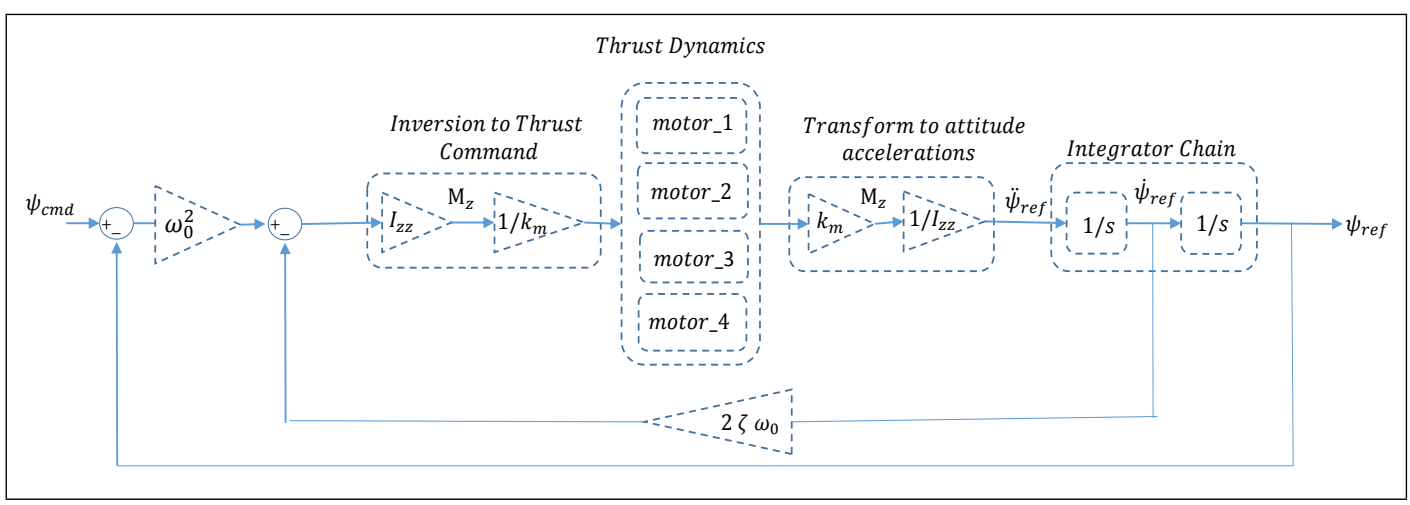

(c) Reference Model Structure for Yaw

Figure 3.3: Structure of the Reference Model

$\mathrm{deg} / \mathrm{s}$ are fed to the reference models for pitch, roll and yaw axes, respectively. The attitude and rate responses of the simulations for various natural frequency values can be observed as seen in Figures 3.4(a) and 3.4(b). Additionally, the responses of error controllers are significant in determining reference model natural frequencies since the gains of error controllers are directly affected by the selection of natural frequency and damping ratio values. Figures $3.4(\mathrm{c}), 3.4(\mathrm{~d})$, $3.4(\mathrm{e})$, and $3.4(\mathrm{f})$ indicate the responses of the error controller to the given step 
input commands. Note that these step input commands are fixed at the maximum error value of $15 \mathrm{deg}$ for yaw axis and $25 \mathrm{deg}$ for pitch and roll axes. The responses in pitch and roll axes can be explored for various integral gains of PID structure in error controller as shown in 3.5 . The case for the state responses in the presence of disturbance is also available in these plots.

\subsection{Pseudo-Control Hedging ( $\mathrm{PCH})$}

This section discusses the implementation of the concept of pseudo control hedging to methodology attained so far. A significant problem for the adaptive control schemes is known as the problem of actuator saturations and may result from actuator position limits, actuator position rate limits and linear input dynamics. In addition to the assumptions, such as affinity and effectiveness in control, the actuator saturations pose a great risk for systems with highly-unstable openloop characteristics (e.g. a quadrocopter) in terms of control authority since all the control possibilities are gathered within the same four actuators. For our case, such a risk is possible when a deviation between the reference model and the system outputs occurs due to saturations. Some approaches that address this were command adjustments, bounding feedback control and the reduction of adaptation rate.

In an attempt to solve this problem, Johnson [138 proposed a modification to system reference models that aims at preventing the adaptive elements of an adaptive control system from attempting to adapt to the system characteristics of the plant or the controller. PCH differs from other methods in the system part where the modification is made. Aforementioned ones modify the adaptive law or the error signals directly, while PCH relies on modifying error signal from within a reference model. Therefore, $\mathrm{PCH}$ protects the adaptive element from problems associated with saturations, hides the system characteristics from adaptive part by moving backwards the reference model, and thus prevents them from seeing the system characteristics as model tracking errors. More clearly, what is desired to be hidden from adaptation is an estimate of the amount the system does not move due to system characteristics. The main motivation in 


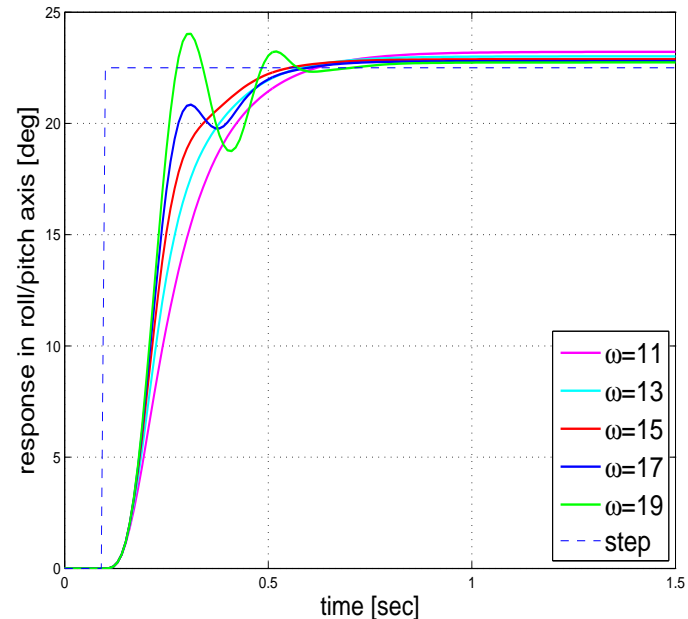

(a) Reference Model Response for Pitch and Roll

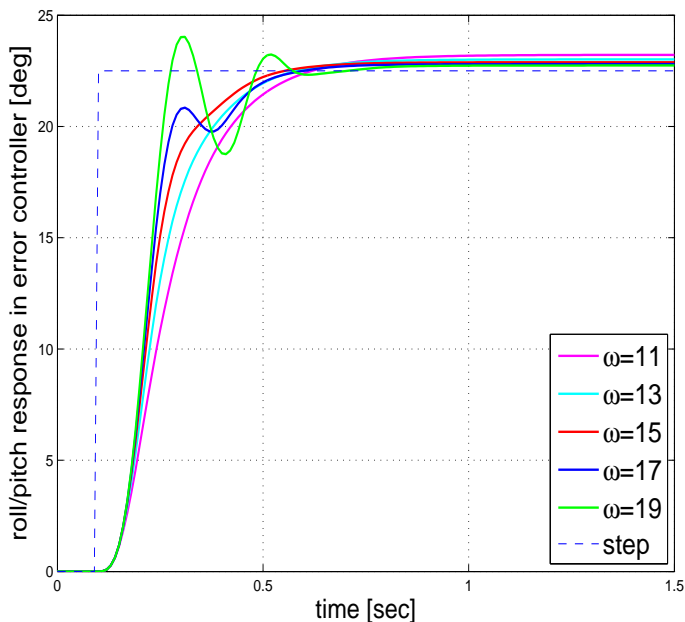

(c) Roll \& Pitch Response in Error Controller

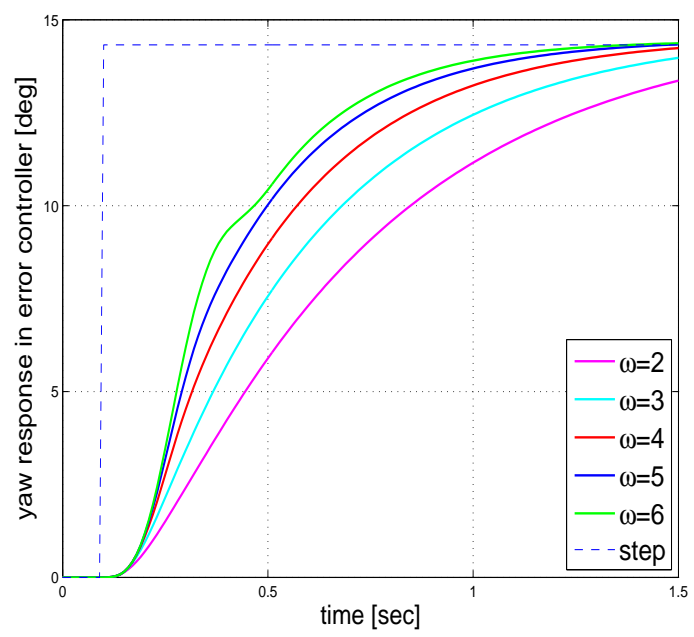

(e) Yaw Response in Error Controller

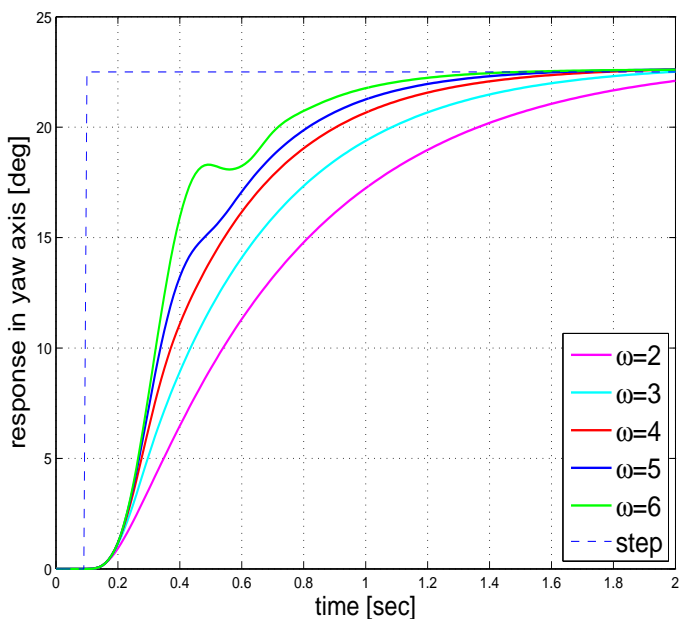

(b) Reference Model Response for Yaw

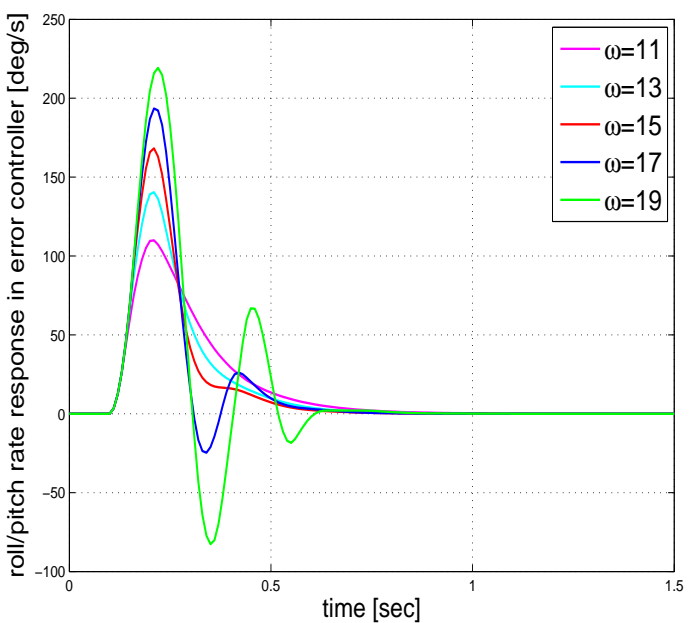

(d) Roll\&Pitch Rate Response in Error Controller

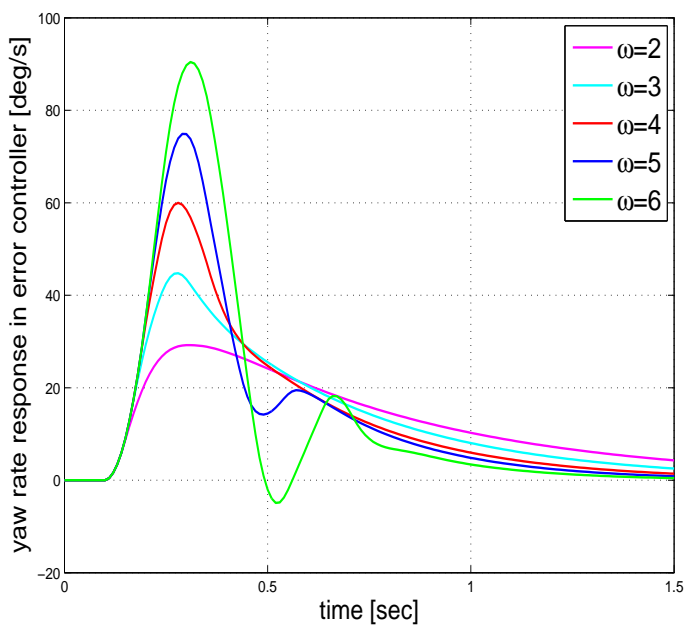

(f) Yaw Rate Response in Error Controller

Figure 3.4: Responses Model and Error Controller State Responses for Various Natural Frequency Values 


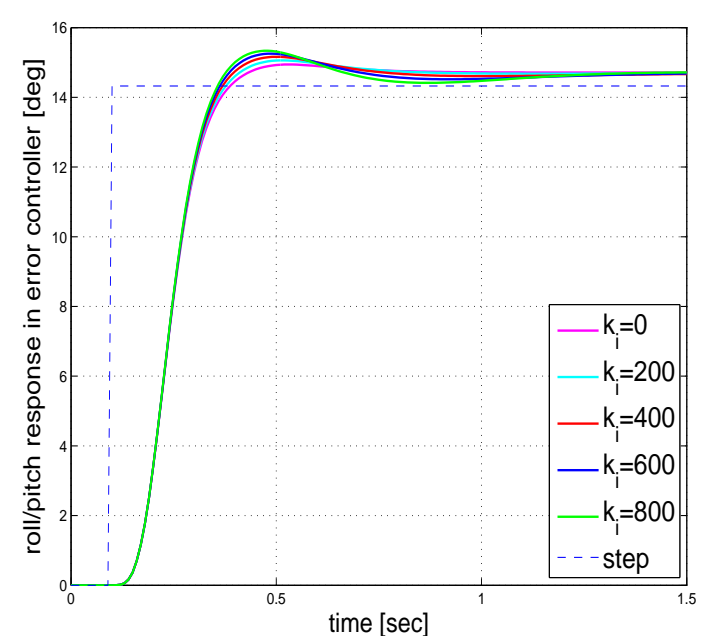

(a) Pitch/Roll Response in Error Controller for Various Integral Gains

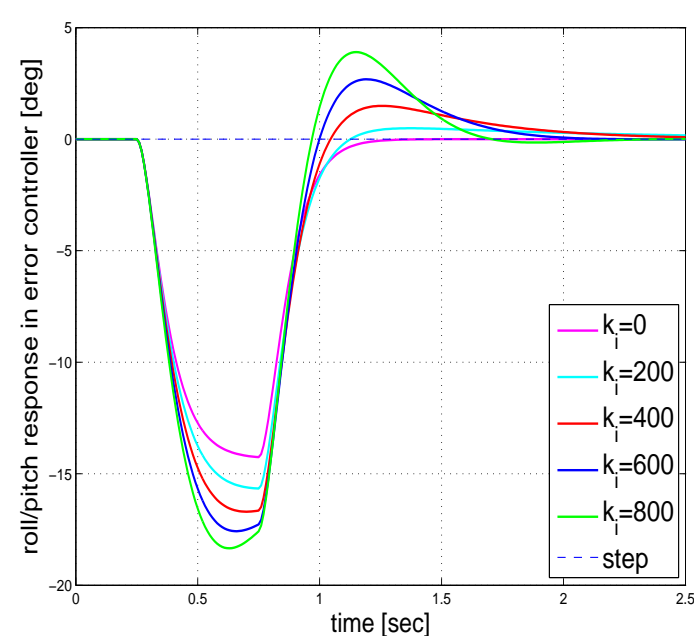

(b) Pitch/Roll Response in Error Controller for Various Integral Gains in the Presence of Unknown Disturbance

Figure 3.5: Error Controller State Responses for Various Integral Gains

quadrotor attitude control in this work is to slow down the reference model exactly as much as the reaction deficit of the command input so as to provide the mentioned hiding.

Let define the difference between the commanded pseudo-control and the achieved pseudo-control (for this case, the reference signal and the plant output) as:

$$
d_{i}=v-\ddot{y}
$$

where $v$ is the reference signal and $\ddot{y}$ is the system output. Note that the reference signal is a function of the states, $x$ and the command input, $u_{c}$ while the system output is based on the actuator dynamics, $D_{\text {act }}$ that changes the command on the system. As stated in [133], the measurements of motor thrusts are not feasible for the controller structure since there occurs a delay even when predicting the thrusts from rotational speeds through a static correlation. Therefore, the estimation of the input can be achieved by using the actuator dynamics and considering the presence of a modelling error as in Eqn. 3.52. Remembering the real thrust input results from the command input and the actuator dynamics, it can be stated that the actuator dynamics should as well be in the estimation 
form:

$$
\begin{gathered}
\ddot{y}=\hat{v}+\Delta=\hat{\ddot{y}}+\Delta \\
\hat{v}=\ddot{y}=-\Delta
\end{gathered}
$$

These estimates are then used to get the final expression for the expected reaction deficiency of the system on the input, $v_{p c h}$ :

$$
\begin{aligned}
& v_{p c h}=v-\hat{v} \\
& v=\hat{v}+v_{p c h}
\end{aligned}
$$

Inserting Eqn.s 3.53 and 3.52 into Eqn. 3.51;

$$
\begin{gathered}
d_{i}=v-\ddot{y}=\hat{v}+v_{p c h}-\hat{v}-\Delta \\
\ddot{y}=v-\hat{v}-v_{p c h}+\hat{v}+\Delta \\
\ddot{y}=v+\Delta-v_{p c h}
\end{gathered}
$$

It should be observed that Eqn. 3.56 is a modification to the structure in Figures 3.3. In such a scheme, the reference input signal is assumed to be equal to the second derivative of the output. The modification in 3.58 can now be applied to Eqn. 3.36 and the resulting relationship can be attained as follows:

$$
\begin{gathered}
\ddot{y}_{r e f}=v_{r e f}-v_{p c h} \\
\ddot{y}_{r e f}=\omega_{0}^{2}\left(y_{c m d}-y_{r e f}\right)-2 \omega_{0} \zeta \dot{y}_{r e f}-v_{p c h}
\end{gathered}
$$

The modified pseudo control output from the error controller is thus as described in Eqn 3.61 for an arbitrary axis:

$$
v_{i}=v_{i, r e f}+k_{d} \dot{e}+k_{p} e+k_{i} e / s-v_{p c h}
$$

Figures 3.6(b) and 3.6(a) summarizes the reference model structure repectively with and without PCH implementation. The last point that should be discussed under this title is the exact equivalence of the error dynamics before and after modification. The second derivative of the output in the reference model in Eqn 3.41a can be employed as follows:

$$
\begin{aligned}
& \ddot{y}_{i}=v_{i}+\Delta_{i}(x) \\
& \ddot{y}_{i}=v_{i, r e f}+k_{d} \dot{e}+k_{p} e+k_{i} e / s-v_{i, p c h}+\Delta_{i}(x)
\end{aligned}
$$




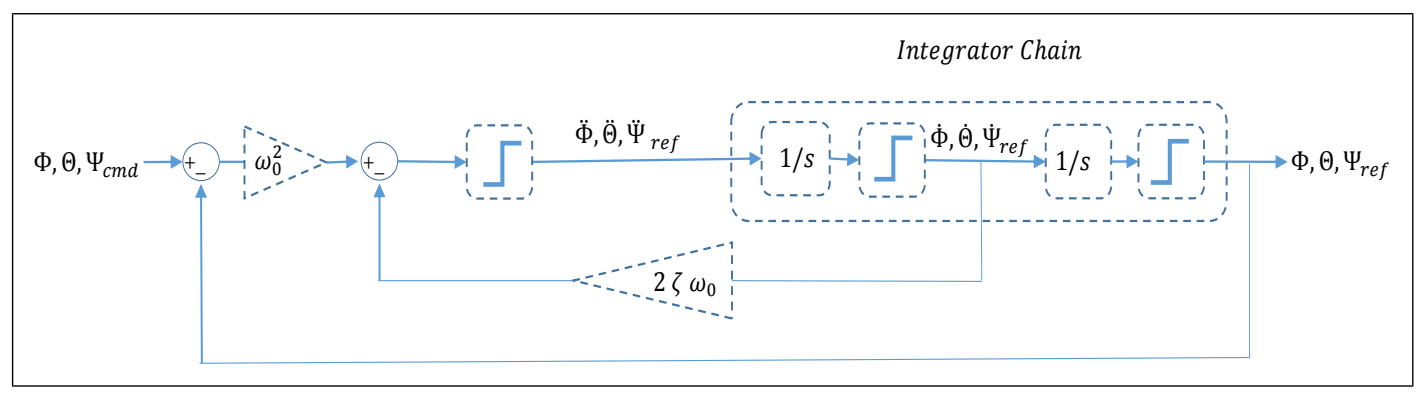

(a) Reference Model Structure without Pseudo-Control Hedging

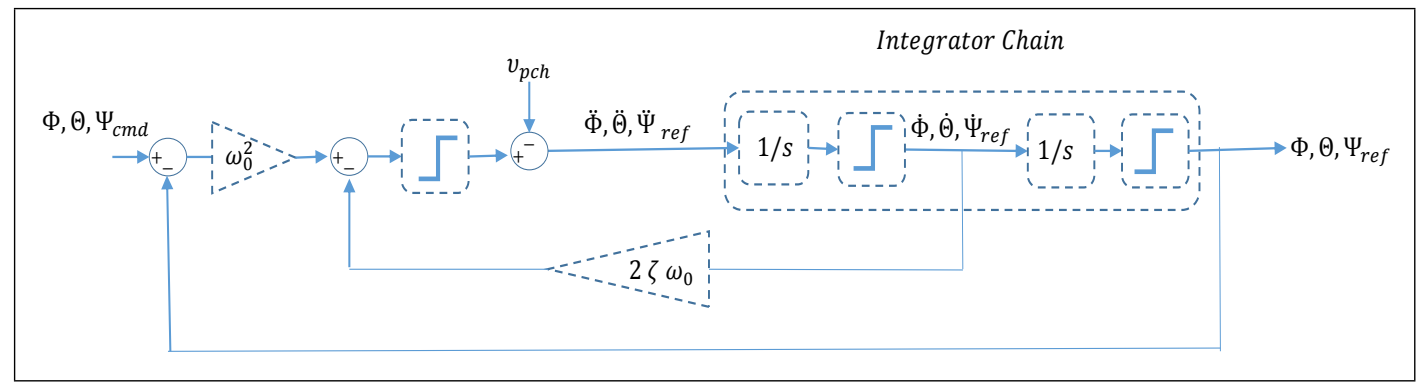

(b) Reference Model Structure with Pseudo-Control Hedging

Figure 3.6: Reference Model Structure with and without Pseudo-Control Hedging

Consider the following relations and then plug them into Eqn $3.62 \mathrm{~b}$

$$
\begin{gathered}
\ddot{e}_{i}=\ddot{y}_{i, r e f}-\ddot{y}_{i} \\
\ddot{y}_{i, r e f}=v_{i, r e f}-v_{i, p c h}
\end{gathered}
$$

Exactly the same equation in Eqn 3.43 is thus achieved:

$$
\ddot{e}+k_{d} \dot{e}+k_{p} e+k_{i} e / s=-\Delta_{i}(x)
$$

In summary, the pseudo control hedging protects the actuator dynamics from the error dynamics. Through the use of integral action in the error controller, another advantage of utilizing pseudo control hedging can be conceived which is the interception of the integrator wind-ups. As a drawback, such a modification still leaves the questions about the guarantee of the stability of the whole reference model unanswered. 


\subsection{Model Reference Adaptive Control}

The identification of geometry or system-based parameters of an aerial platform may require exhausting identification or experimentation. Using a controller with the competence to identify the unknown parameters or to resist parameter changes provides robustness by adding adaptive elements. The use of Model Reference Adaptive Control (MRAC) is motivated by this fact. Within the concept of a quadrocopter flight, the adaptation process may include the system inputs, the controller error and some other specific variables, and in this manner, this method targets at serving as a compensator for the uncertainties of the moment of inertia and motor and propeller related parameters of the system.

The general trend in the implementation of an adaptive controller to a quadrotor system is that the plant dynamics is allocated into well-known non-linear and unknown parameters which are often quite expensive to obtain through experiments for small scale UAVs and thus matched by adaptive methods.

MRACs depends on a reference model scheme that matches the dynamics of the platform while adding adaptive elements to the procedure. To have a deeper understanding of the idea of MRAC, some noteworthy points to touch on are the difference of adaptation from a well-known Model Reference Control (MRC) approach and the direct and indirect types of MRAC as explained in [139. In $\mathrm{MRC}$, the desired plant behaviour is described by a reference model, a reference input and the control law which are developed so that the closed-loop characteristics match the plant behaviour. Figure 3.7 depicts MRC. If the unknown

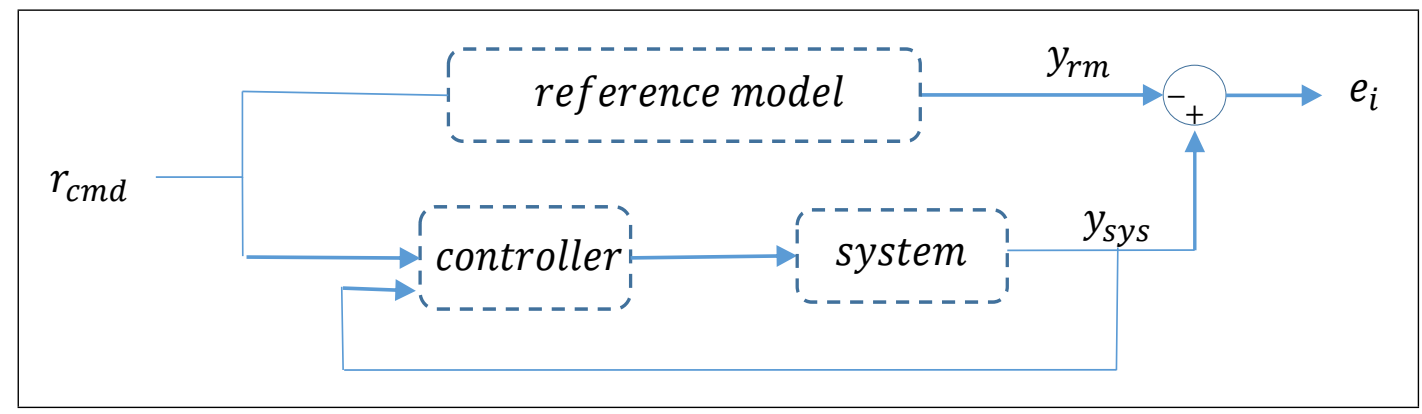

Figure 3.7: The General Structure of MRC 


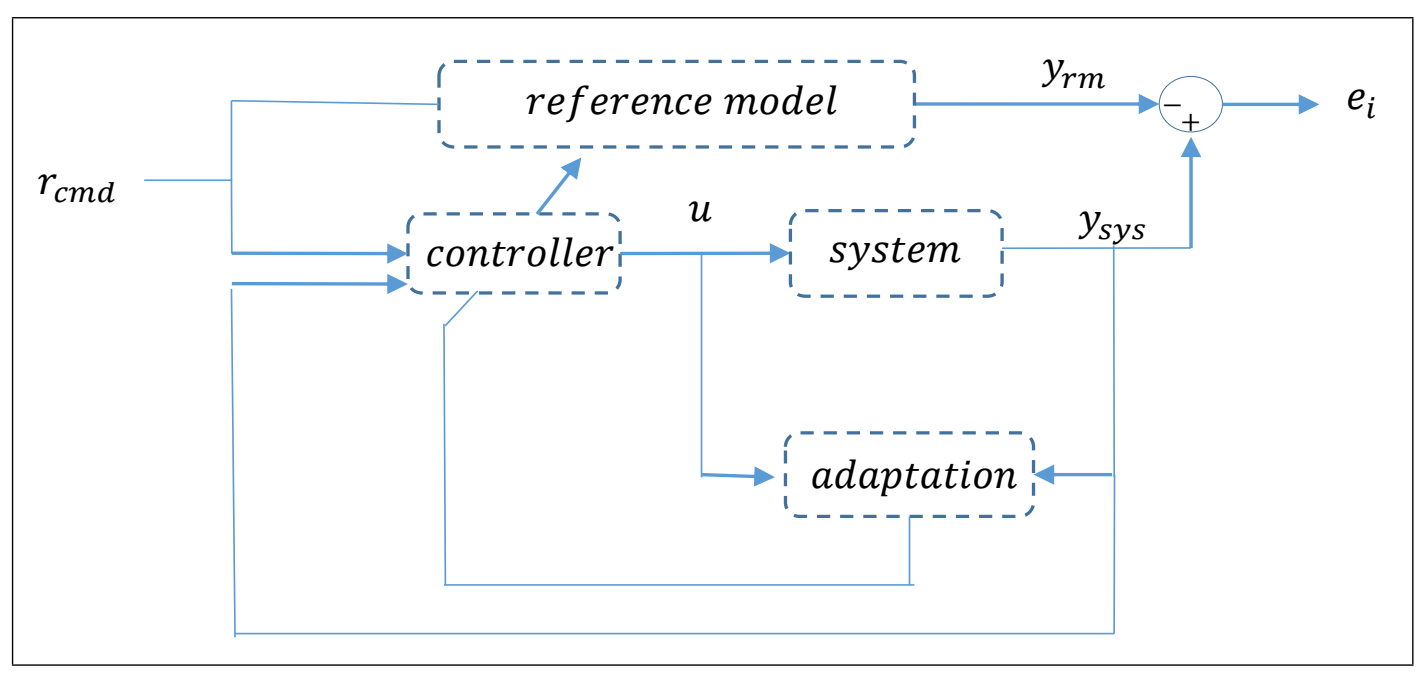

Figure 3.8: The General Structure of MRAC

plant parameters are estimated and the controller parameters are calculated using these estimations at each time step, such an MRAC is defined as "indirect MRAC". The direct approach for MRACs adapts the controller to compensate for the unknown plant parameters. The derivation of these adaptations and the stability of the closed loop are achieved through Lyapunov analysis. In this scheme, the plant characteristics are parametrized in terms of the desired controller parameter vector, $\Theta_{c}$. The general MRAC approach is illustrated in Fig. 3.8 In direct MRAC scheme, the controller error is defined as the difference between the outputs of the system and the reference model. The adaptive controller adjustment is accomplished by the employment of this controller error as well as the system inputs. As the limitation of any controller, the dynamics of the adaptive controller is restricted to be slower than that of the real plant. Contrary to the structural adaptive methods like neural networks that approximate the unknown non-linear functions, it is essential to separate the plant dynamics into well-known non-linear functions and unknown parameters in a parameter adaptation scheme. For this specific research, using the analogy achieved by Holzapfel [134] and the studies conducted on quadrotors by Achtelik [133], the controller is separated into two parts, namely, Adaptive Rate and Attitude Controller loops. Figure 3.9 indicates this cascaded controller structure. 


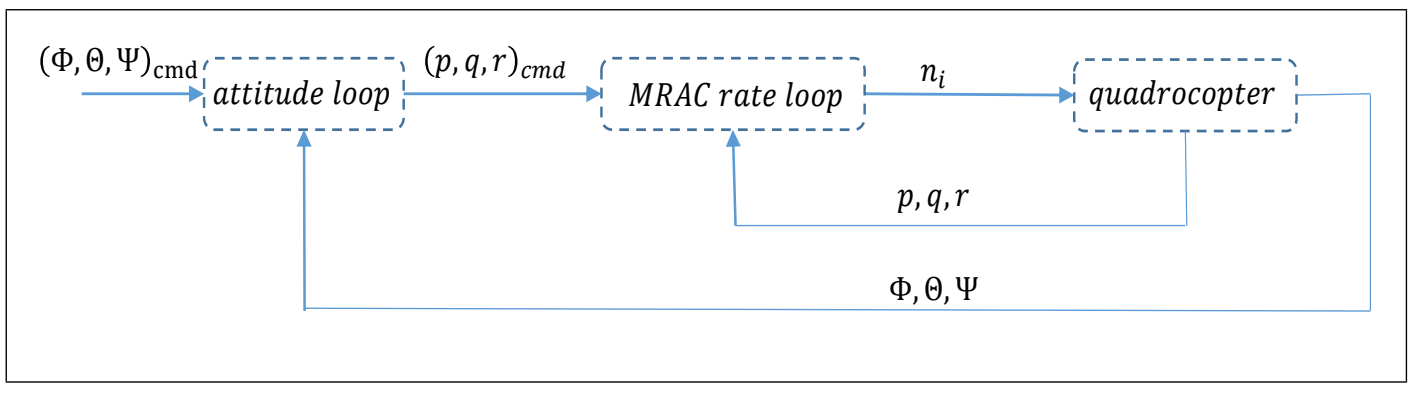

Figure 3.9: Modified Cascaded Controller Structure for Adaptive Laws

\subsubsection{Adaptive Rate Controller within MRAC Structure}

Inside the adaptive rate controller, the angular momentum equation should be considered. In this manner, the states are chosen as the body angular rate $p, q$ and $r$. The trigonometric relation from the Euler angle commands to body rate commands are then separated from the adaptive rate loop. The state equation inside this rate loop can be obtained from Eqn. 2.40 as in Eqn. 3.65 .

$$
\dot{\vec{\omega}}=I^{-1} \cdot \vec{M}-I^{-1} \cdot[\vec{\omega} \times I \vec{\omega}]
$$

Expanding in matrix form:

$$
\left[\begin{array}{c}
\dot{p} \\
\dot{q} \\
\dot{r}
\end{array}\right]=\left[\begin{array}{ccc}
I_{x x} & 0 & 0 \\
0 & I_{y y} & 0 \\
0 & 0 & I_{z z}
\end{array}\right]^{-1}\left[\begin{array}{c}
M_{x} \\
M_{y} \\
M_{z}
\end{array}\right]-\left[\begin{array}{ccc}
I_{x x} & 0 & 0 \\
0 & I_{y y} & 0 \\
0 & 0 & I_{z z}
\end{array}\right]^{-1}\left[\begin{array}{l}
p \\
q \\
r
\end{array}\right] \times\left[\begin{array}{ccc}
I_{x x} & 0 & 0 \\
0 & I_{y y} & 0 \\
0 & 0 & I_{z z}
\end{array}\right]\left[\begin{array}{l}
p \\
q \\
r
\end{array}\right]
$$

Inserting Eqn. 2.17 in,

$$
\left[\begin{array}{c}
\dot{p} \\
\dot{q} \\
\dot{r}
\end{array}\right]=\left[\begin{array}{ccc}
I_{x x}^{-1} & 0 & 0 \\
0 & I_{y y}^{-1} & 0 \\
0 & 0 & I_{z z}^{-1}
\end{array}\right]\left(\left[\begin{array}{cccc}
0 & -r & 0 & r \\
r & 0 & -r & 0 \\
-k_{m} & k_{m} & -k_{m} & k_{m}
\end{array}\right]\left[\begin{array}{l}
F_{1} \\
F_{2} \\
F_{3} \\
F_{4}
\end{array}\right]-\left[\begin{array}{l}
\left(I_{z}-I_{y}\right) q r \\
\left(I_{x}-I_{z}\right) p r \\
\left(I_{y}-I_{x}\right) p q
\end{array}\right]\right)
$$


If the multiplication of body rates are defined as another non-linear elements vector, the matrices turn into Eqn. 3.68 .

$$
\left[\begin{array}{l}
\dot{p} \\
\dot{q} \\
\dot{r}
\end{array}\right]=\left[\begin{array}{cccc}
0 & \frac{-r}{I_{x x}} & 0 & \frac{r}{I_{x x}} \\
\frac{r}{I_{y y}} & 0 & \frac{-r}{I_{y y}} & 0 \\
\frac{-k_{m}}{I_{z z}} & \frac{k_{m}}{I_{z z}} & \frac{-k_{m}}{I_{z z}} & \frac{k_{m}}{I_{z z}}
\end{array}\right]\left[\begin{array}{c}
F_{1} \\
F_{2} \\
F_{3} \\
F_{4}
\end{array}\right]-\left[\begin{array}{ccc}
\frac{I_{z z}-I_{y y}}{I_{x x}} & 0 & 0 \\
0 & \frac{I_{x x}-I_{z z}}{I_{y y}} & 0 \\
0 & 0 & \frac{I_{y y}-I_{x x}}{I_{z z}}
\end{array}\right]\left[\begin{array}{l}
q r \\
p r \\
p q
\end{array}\right]
$$

Investigating this expression, it is clear that the equation is composed of a nonlinear part and a control input part. Remembering that adaptiveness is related to parameters like moments of inertia at this point, Eqn. 3.68 can be extended with the linear part of uncertain dynamics and a constant disturbance vector.

$$
\dot{x}_{p}=A_{p} x_{p}+B_{p} \Lambda u+\alpha_{p} f\left(x_{p}\right)+d
$$

Thanks to this deduction, the state equation for the system is obtained. Next, with an aim to achieve the identification and the compensation of the matrices which contain uncertain parameters, an adaptive control law can be deduced such that the dynamics of the reference model matches the characteristics of the plant output dynamics. Finally, the adaptive control law in the adaptation matrices that represent the parameter uncertainties can be used.

$$
u=\Theta_{x} x_{p}+\Theta_{r} x_{r}+\Theta_{\alpha} f\left(x_{p}\right)+\Theta_{d} i
$$

where $A_{p}, \alpha_{p}$ and $d$ are the uncertain parameters to be compensated; $r$ are the commands of body angular rates and $\Theta_{x, r, d, \alpha}$ are adaptive gains. Insert Eqn. 3.70 into Eqn. 3.69 .

$$
\begin{gathered}
\dot{x}_{p}=A_{p} x_{p}+B_{p} \Lambda\left(\Theta_{x} x_{p}+\Theta_{r} x_{r}+\Theta_{\alpha} f\left(x_{p}\right)+\Theta_{d} i\right)+\alpha_{p} f\left(x_{p}\right)+d \\
\dot{x}_{p}=\left(A_{p}+B_{p} \Lambda \Theta_{x}\right) x_{p}+B_{p} \Lambda \Theta_{r} r+\left(B_{p} \Lambda \Theta_{\alpha}+\alpha_{p}\right) f\left(x_{p}\right)+\left(B_{p} \Lambda \Theta_{d}+D\right) i \\
\dot{x}_{p}=A_{\tilde{p}} x_{p}+B_{\tilde{p}} r+\alpha_{\tilde{p}} f\left(x_{p}\right)+D_{\tilde{p}} i
\end{gathered}
$$

The extended rate adaptive controller structure can be investigated in Figure 3.10 as visualized in [133]. The defined structure makes use of a first order reference model:

$$
\dot{x}_{M}=A_{M} x_{M}+B_{M} r
$$




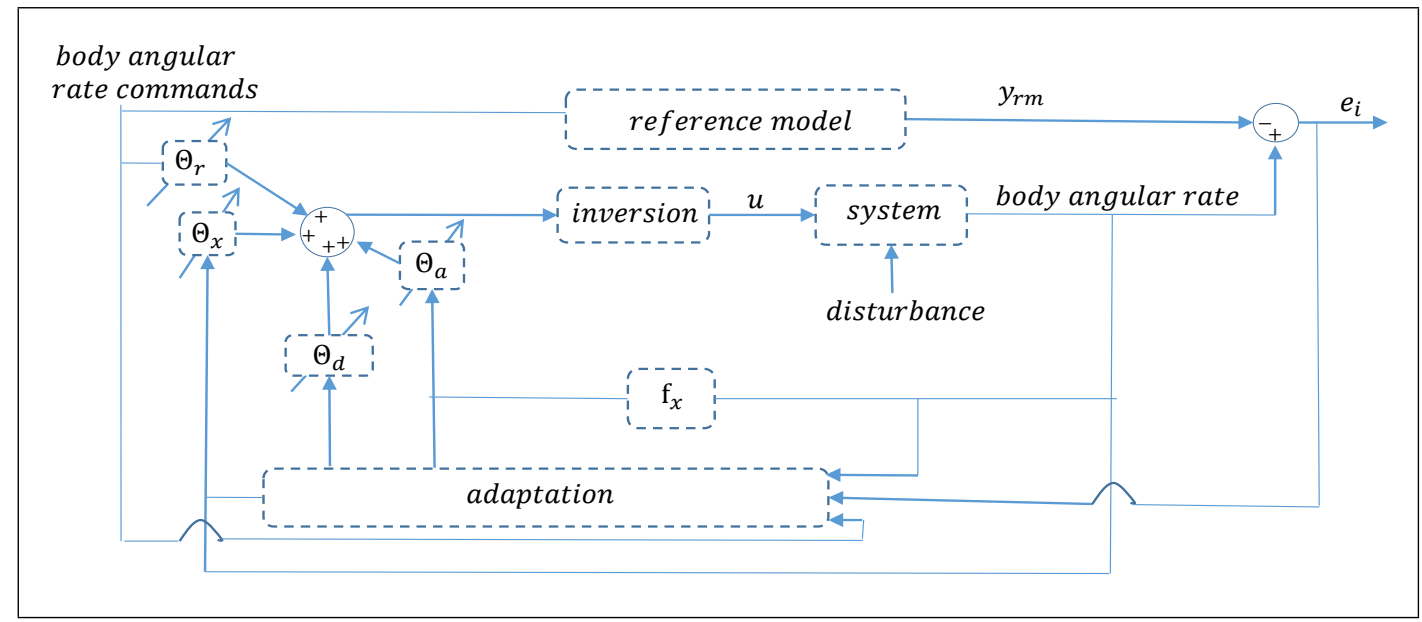

Figure 3.10: Model Reference Adaptive Controller Structure with Adaptive Gains

where $x_{M}=\left[\begin{array}{lll}p & q & r\end{array}\right]^{T}, r=\left[\begin{array}{lll}p_{c m d} & q_{c m d} & r_{c m d}\end{array}\right]^{T}$. The matrices $A_{M}$ and $B_{M}$ are determined as in Eqn.s $3.75 \mathrm{a}$ and $3.75 \mathrm{~b}$ so as to maintain the stability.

$$
\begin{aligned}
A_{M}= & {\left[\begin{array}{ccc}
-1 / T_{p} & 0 & 0 \\
0 & -1 / T_{q} & 0 \\
0 & 0 & -1 / T_{r}
\end{array}\right] } \\
B_{M}= & {\left[\begin{array}{ccc}
1 / T_{p} & 0 & 0 \\
0 & 1 / T_{q} & 0 \\
0 & 0 & 1 / T_{r}
\end{array}\right] }
\end{aligned}
$$

Assume that the plant matches the reference dynamics with the ideal gain matrices, $\Theta_{x}^{*}, \Theta_{\alpha}^{*}, \Theta_{r}^{*}$ and $\Theta_{d}^{*}$.

$$
\begin{gathered}
A_{M}=A_{\tilde{p}}=A_{p}+B_{p} \Lambda \Theta_{x}^{*} \\
B_{M}=B_{\tilde{p}}=B_{p} \Lambda \Theta_{r}^{*}
\end{gathered}
$$

Also note that the terms related to non-linearities and disturbances should be zero:

$$
\begin{gathered}
0=\alpha_{\tilde{p}} \Rightarrow B_{p} \Lambda \Theta_{\alpha}^{*}+\alpha_{p}=0 \\
0=d_{\tilde{p}} \Rightarrow B_{p} \Lambda \Theta_{d}^{*}+D=0
\end{gathered}
$$


Combining all these knowledge, following relations can be derived.

$$
\begin{aligned}
A_{p} & =A_{M}-B_{p} \Lambda \Theta_{x}^{*} \\
B_{M} & =B_{p} \Lambda \Theta_{r}^{*} \\
\alpha_{p} & =-B_{p} \Lambda \Theta_{\alpha}^{*} \\
D & =-B_{p} \Lambda \Theta_{d}^{*}
\end{aligned}
$$

Since the difference between the reference model and the plant is the criteria for the matching, this difference can be defined as the control error. The error dynamics can be found just by looking at its first derivative.

$$
\begin{aligned}
e_{a c} & =x_{p}-x_{M} \\
\dot{e}_{m r a c} & =\dot{x}_{P}-\dot{x}_{M}
\end{aligned}
$$

Inserting in the equations 3.74 and 3.72 into $3.80 \mathrm{~d}$, Eqn 3.82 can be attained.

$$
\begin{aligned}
e_{a c}=\left(A_{p}+B_{p} \Lambda \Theta_{x}\right) x_{p}+B_{p} \Lambda \Theta_{r} r & +\left(B_{p} \Lambda \Theta_{\alpha}+\alpha_{p}\right) f\left(x_{p}\right) \\
& +\left(B_{p} \Lambda \Theta_{d}+D\right) i-\left(A_{M} x_{M}+B_{M} r\right)
\end{aligned}
$$

Also, inserting in Eqn 3.80a, 3.80b, 3.80c and 3.80d,

$$
\begin{aligned}
e_{a c}=( & \left.A_{m}-B_{p} \Lambda \Theta_{x}^{*}+B_{p} \Lambda \Theta_{x}\right) x_{p}+B_{p} \Lambda \Theta_{r} r \\
& +\left(B_{p} \Lambda \Theta_{\alpha}+\alpha_{p}\right) f\left(x_{p}\right)+\left(B_{p} \Lambda \Theta_{d}+D\right) i-\left(A_{m} x_{M}+B_{p} \Lambda \Theta_{r}^{*} r\right)
\end{aligned}
$$

Arrange Eqn 3.83 in the following form:

$$
\begin{aligned}
e_{a c}=A_{m}\left(x_{p}-x_{m}\right)+B_{p} \Lambda\left(\Theta_{x}\right. & \left.-\Theta_{x}^{*}\right) x_{p}+B_{p} \Lambda\left(\Theta_{r}-\Theta_{r}^{*}\right) r \\
& +B_{p} \Lambda\left(\Theta_{\alpha}-\Theta_{\alpha}^{*}\right) f\left(x_{p}\right)+B_{p} \Lambda\left(\Theta_{d}-\Theta_{d}^{*}\right) i
\end{aligned}
$$

The subtractions inside the parenthesis in Eqn 3.84 can be defined as the errors between the ideal and the attained parameters.

$$
\begin{aligned}
& \tilde{\Theta}_{x}=\Theta_{x}-\Theta_{x}^{*} \\
& \tilde{\Theta}_{r}=\Theta_{r}-\Theta_{r}^{*} \\
& \tilde{\Theta}_{\alpha}=\Theta_{\alpha}-\Theta_{\alpha}^{*} \\
& \tilde{\Theta}_{d}=\Theta_{d}-\Theta_{d}^{*}
\end{aligned}
$$

Paying attention to Eqn $3.81 \mathrm{a}$ as well, the final form of error dynamics is obtained in Eqn 3.86 which is actually the fundamental law for adaptation.

$$
\dot{e}_{a c}=A_{m} e_{a c}+B_{p} \Lambda \tilde{\Theta}_{x} x_{p}+B_{p} \Lambda \tilde{\Theta}_{r} r+B_{p} \Lambda \tilde{\Theta}_{\alpha} f\left(x_{p}\right)+B_{p} \Lambda \tilde{\Theta}_{d} i
$$




\subsubsection{Lyapunov's Direct Method}

The discussion on the stability of the dynamic systems described by differential equations by the theory of Lyapunov is not uncommon. Similarly, in order to determine whether an adaptive system is stable or not, Lyapunov's direct method is a good point to start. The basic idea behind this method is that the continuous dissipation of the total energy of a system causes it to settle down to an equilibrium. The following definitions and theorems are well explained in [140]. Let $\Omega \subseteq R^{n}$ be an open set containing the origin. Assume a continuously differentiable function $f$ such that $f: R \times \Omega \rightarrow R^{n}$ and $f(0)=0 \forall t$ so that $x(t)=0$ is a solution to the differential equation $\dot{x}=f(x)$. Suppose $\mathrm{D}$ is a neighbourhood of origin within $\Omega$ and the scalar function, $W: D \rightarrow R$, is continuous and initially zero. Then, Defn. 11 can be deduced.

\section{Definition 1.}

$$
W \begin{cases}\text { positive semi definite } & \text { if } W(x) \geq 0 \\ \text { negative semi definite } & \text { if } W(x) \leq 0 \\ \text { positive definite } & \text { if } W(x)>0 \\ \text { negative definite } & \text { if } W(x)<0\end{cases}
$$

Additionally, suppose that there exist continuous scalar function, $V: R \times D \rightarrow$ $R$, satisfying $V=0 \forall t \in R$. Then,

\section{Definition 2.}

$V \begin{cases}\text { pos.s.d. } & \text { if } \exists \text { a pos.s.d. } W: D \rightarrow R \text { s.t. } V(x, t) \geq W(x) \forall(t, x) \in R \times D \\ \text { pos.def. } & \text { if } \exists \text { a pos.def. } W: D \rightarrow R \text { s.t. } V(x, t) \geq W(x) \forall(t, x) \in R \times D \\ \text { neg.s.d. } & \text { if } \exists \text { a neg.s.d. } W: D \rightarrow R \text { s.t. } V(x, t) \leq W(x) \forall(t, x) \in R \times D \\ \text { neg.def. } & \text { if } \exists \text { a neg.def. } W: D \rightarrow R \text { s.t. } V(x, t) \leq W(x) \forall(t, x) \in R \times D\end{cases}$

If $V: R \times D$ is continuously differentiable, then $\dot{V}: R \times D$ exists. The following theorems summarize the Lyapunov Stability analysis used in this study [135].

Theorem 1. If $\exists$ a neighbourhood $D$ around the origin and a continuously differentiable positive definite function $V: R \times D \rightarrow R$ whose time derivative is negative semi-definite, then this origin is a Lyapunov stable solution of the differential equation representing the system. 
Theorem 2. Let $\exists$ a neighbourhood $D$ around the origin and a continuously differentiable positive definite function $V: R \times D \rightarrow R$ whose time derivative is negative semi-definite. Suppose that $\exists$ a positive definite function $\tilde{W}: d \rightarrow R$ such that $V(x, t) \leq \tilde{W}(x) \forall(t i x) \in R \times D$. Then, this origin is an asymptotically stable solution of the differential equation representing the system.

Theorem 3. Every function satisfying positive definiteness in a neighbourhood $D$ excluding the origin and having negative semi definite time derivative (i.e. a stable origin point) is called a Lyapunov function.

The overall purpose of Lyapunov's Direct method is to choose a suitable Lyapunov function to ensure the stability of the system. Also note that one cannot make a deduction just by finding a positive definite time derivative since there may exist another Lyapunov function which results in a stable and even asymptotically stable system.

\subsubsection{Lyapunov Analysis for Adaptive Rate Controller}

For adaptive systems, the utilization of a Lyapunov function of type in Eqn 3.87 , is explained in [141]. Note that $P, \Lambda$ and $\Upsilon$ are positive definite symmetric matrices and $\operatorname{Tr}$ designates trace operation.

$$
V=\frac{1}{2} z_{1}^{T} P z_{1}+\frac{1}{2} \operatorname{Tr}_{2}^{T} \Upsilon^{-1} z_{2}|\Lambda|
$$

Following the same analogy, adaptive control error and the differences between the ideal and the real parameters can be chosen as variables. The error variable applies to the first element of Eqn. 3.87, while the differences in Eqn.s 3.85a, $3.85 \mathrm{~b}$, 3.85c and 3.85d apply to the trace elements. The Lyapunov function then becomes Eqn. 3.88 .

$$
\begin{aligned}
V=\frac{1}{2} e_{a c}^{T} P e_{a c}+\frac{1}{2} \operatorname{Tr}\left[\tilde{\Theta}_{x}^{T} \Upsilon_{x}^{-1} \tilde{\Theta}_{x}|\Lambda|\right] & +\frac{1}{2} \operatorname{Tr}\left[\tilde{\Theta}_{r}^{T} \Upsilon_{r}^{-1} \tilde{\Theta}_{r}|\Lambda|\right] \\
& +\frac{1}{2} \operatorname{Tr}\left[\tilde{\Theta}_{\alpha}^{T} \Upsilon_{\alpha}^{-1} \tilde{\Theta}_{\alpha}|\Lambda|\right]+\frac{1}{2} \operatorname{Tr}\left[\tilde{\Theta}_{d}^{T} \Upsilon_{d}^{-1} \tilde{\Theta}_{d}|\Lambda|\right]
\end{aligned}
$$

In Eqn 3.88 , it is clear due to the positive definiteness and symmetry of $\mathrm{P}$ and $\Upsilon$ matrices that the function is positive definite. In order to apply Lyapunov's direct method to find out whether the chosen Lyapunov function brings stability 
to adaptation or not, the time-derivative of this Lyapunov function should be evaluated as in Eqn. 3.90. Before the evaluation, the time derivative of the trace element is expressed in Eqn 3.89

$$
\begin{aligned}
& \frac{1}{2} \frac{d}{d t}\left[\operatorname{Tr}\left[\tilde{\Theta}^{T} \Upsilon^{-1} \tilde{\Theta}|\Lambda|\right]\right]=\frac{1}{2} \operatorname{Tr}\left[\dot{\tilde{\Theta}}^{T} \Upsilon^{-1} \tilde{\Theta}|\Lambda|\right]+\frac{1}{2} \operatorname{Tr}\left[\tilde{\Theta}^{T} \Upsilon^{-1} \dot{\tilde{\Theta}}|\Lambda|\right]=\operatorname{Tr}\left[\tilde{\Theta}^{T} \Upsilon^{-1} \dot{\tilde{\Theta}}|\Lambda|\right] \\
& \dot{V}=\frac{1}{2}\left[e_{a c}{ }^{T} P e_{a c}+e_{a c}^{T} P \dot{e}_{a c}^{\cdot}\right]+\operatorname{Tr}\left[\tilde{\Theta}_{x}^{T} \Upsilon_{x}^{-1} \dot{\tilde{\Theta}}_{x}|\Lambda|\right]+\operatorname{Tr}\left[\tilde{\Theta}_{r}^{T} \Upsilon_{r}^{-1} \dot{\tilde{\Theta}}_{r}|\Lambda|\right] \\
& +\operatorname{Tr}\left[\tilde{\Theta}_{\alpha}^{T} \Upsilon_{\alpha}^{-1} \dot{\tilde{\Theta_{\alpha}}}|\Lambda|\right]+\operatorname{Tr}\left[\tilde{\Theta}_{d}^{T} \Upsilon_{d}^{-1} \dot{\tilde{\Theta}}_{d}|\Lambda|\right]
\end{aligned}
$$

where

$$
\dot{e}_{a c}^{T} P e_{a c}=\left(e_{a c}^{T} P \dot{e}_{a c}\right)^{T}
$$

Now consider:

$$
\begin{aligned}
& e_{a c}^{T} P \dot{e}_{a c}=e_{a c}^{T} P\left[A_{m} e_{a c}+B_{p} \Lambda \tilde{\Theta}_{x} x_{p}+B_{p} \Lambda \tilde{\Theta}_{r} r+B_{p} \Lambda \tilde{\Theta}_{\alpha} f\left(x_{p}\right)+B_{p} \Lambda \tilde{\Theta}_{d} i\right] \\
& \dot{e}_{a c}^{T} P e_{a c}=\left[A_{m} e_{a c}+B_{p} \Lambda \tilde{\Theta}_{x} x_{p}+B_{p} \Lambda \tilde{\Theta}_{r} r+B_{p} \Lambda \tilde{\Theta}_{\alpha} f\left(x_{p}\right)+B_{p} \Lambda \tilde{\Theta}_{d} i\right]^{T} P e_{a c}
\end{aligned}
$$

The summation of 3.92 and 3.93 leads to 3.94

$$
\begin{gathered}
e_{a c}^{T} P \dot{e}_{a c}+\dot{e}_{a c}^{T} P e_{a c}=e_{a c}^{T} P A_{m} e_{a c}+e_{a c}^{T} A_{m}^{T} P e_{a c}+e_{a c}^{T} P B_{p} \Lambda \tilde{\Theta}_{x} x_{p} \\
+x_{p}^{T} \tilde{\Theta}_{x}^{T} \Lambda^{T} B_{p}^{T} P e_{a c}+e_{a c}^{T} P B_{p} \Lambda \tilde{\Theta}_{r} r+r^{T} \tilde{\Theta}_{r}^{T} \Lambda^{T} B_{p}^{T} P e_{a c}+e_{a c}^{T} P B_{p} \Lambda \tilde{\Theta}_{\alpha} f\left(x_{p}\right) \\
+f\left(x_{p}\right)^{T} \tilde{\Theta}_{\alpha}^{T} \Lambda^{T} B_{p}^{T} P e_{a c}+e_{a c}^{T} P B_{p} \Lambda \tilde{\Theta}_{d} i+i^{T} \tilde{\Theta}_{d}^{T} \Lambda^{T} B_{p}^{T} P e_{a c}
\end{gathered}
$$

First of all, the first term of Eqn 3.95 should be negative definite in order to have a negative definite $\dot{V}$. This can be achieved by Eqn 3.96 where $Q$ is assumed to be a symmetrical positive definite matrix.

$$
P A_{m}+A_{m}^{T} P=-Q
$$


For other adaptation matrix terms, the following relationships in Eqn.s 3.97a, $3.97 \mathrm{~b}, 3.97 \mathrm{c}$ and $3.97 \mathrm{~d}$ can be written.

$$
\begin{aligned}
& x_{p}^{T} \tilde{\Theta}_{x}^{T} \Lambda^{T} B_{p}^{T} P e_{a c}=\operatorname{Tr}\left[\tilde{\Theta}_{x}^{T} \Lambda^{T} B_{p}^{T} P e_{a c} x_{p}^{T}\right] \\
& r^{T} \tilde{\Theta}_{r}^{T} \Lambda^{T} B_{p}^{T} P e_{a c}=\operatorname{Tr}\left[\tilde{\Theta}_{r}^{T} \Lambda^{T} B_{p}^{T} P e_{a c} r^{T}\right] \\
& f^{T} \tilde{\Theta}_{\alpha}^{T} \Lambda^{T} B_{p}^{T} P e_{a c}=\operatorname{Tr}\left[\tilde{\Theta}_{\alpha}^{T} \Lambda^{T} B_{p}^{T} P e_{a c} f^{T}\right] \\
& i^{T} \tilde{\Theta}_{d}^{T} \Lambda^{T} B_{p}^{T} P e_{a c}=\operatorname{Tr}\left[\tilde{\Theta}_{d}^{T} \Lambda^{T} B_{p}^{T} P e_{a c} i^{T}\right]
\end{aligned}
$$

Through these derivations, Eqn. 3.95 can be rewritten.

$$
\begin{aligned}
& \frac{1}{2}\left[e_{a c}^{T} P \dot{e}_{a c}+\dot{e}_{a c}^{T} P e_{a c}\right]=-\frac{1}{2} e_{a c}^{T} Q e_{a c}+\operatorname{Tr}\left[\tilde{\Theta}_{x}^{T} \Lambda^{T} B_{p}^{T} P e_{a c} x_{p}^{T}\right] \\
& \quad+\operatorname{Tr}\left[\tilde{\Theta}_{r}^{T} \Lambda^{T} B_{p}^{T} P e_{a c} r^{T}\right]+\operatorname{Tr}\left[\tilde{\Theta}_{\alpha}^{T} \Lambda^{T} B_{p}^{T} P e_{a c} f^{T}\right]+\operatorname{Tr}\left[\tilde{\Theta}_{d}^{T} \Lambda^{T} B_{p}^{T} P e_{a c} i^{T}\right]
\end{aligned}
$$

Inserting Eqn. 3.98 into Eqn. 3.90,

$$
\begin{aligned}
\dot{V}= & -\frac{1}{2} e_{a c}^{T} Q e_{a c} \\
& +\operatorname{Tr}\left[\tilde{\Theta}_{x}^{T} \Lambda^{T}\left(B_{p}^{T} P e_{a c} x_{p}^{T}+\Upsilon_{x}^{-1} \dot{\tilde{\Theta}}_{x}\right)\right] \\
& +\operatorname{Tr}\left[\tilde{\Theta}_{r}^{T} \Lambda^{T}\left(B_{p}^{T} P e_{a c} r^{T}+\Upsilon_{r}^{-1} \dot{\tilde{\Theta}}_{r}\right)\right] \\
& +\operatorname{Tr}\left[\tilde{\Theta}_{\alpha}^{T} \Lambda^{T}\left(B_{p}^{T} P e_{a c} f^{T}+\Upsilon_{\alpha}^{-1} \dot{\tilde{\Theta}}_{\alpha}\right)\right] \\
& +\operatorname{Tr}\left[\tilde{\Theta}_{d}^{T} \Lambda^{T}\left(B_{p}^{T} P e_{a c} i^{T}+\Upsilon_{d}^{-1} \dot{\tilde{\Theta}}_{d}\right)\right]
\end{aligned}
$$

As mentioned before, for the system to be stable, $\dot{V}$ term should be negative definite or semi definite. By defining $Q$ as positive definite matrix and equating all trace elements to zero, this aim can be accomplished as shown in Eqn.s $3.100 \mathrm{a}, 3.100 \mathrm{~b}, 3.100 \mathrm{c}$ and $3.100 \mathrm{~d}$.

$$
\begin{array}{r}
\tilde{\Theta}_{x}^{T} \Lambda^{T}\left(B_{p}^{T} P e_{a c} x_{p}^{T}+\Upsilon_{x}^{-1} \dot{\tilde{\Theta_{x}}}\right)=0 \\
\tilde{\Theta}_{r}^{T} \Lambda^{T}\left(B_{p}^{T} P e_{a c} r^{T}+\Upsilon_{r}^{-1} \dot{\tilde{\Theta}}_{r}\right)=0 \\
\tilde{\Theta}_{\alpha}^{T} \Lambda^{T}\left(B_{p}^{T} P e_{a c} f^{T}+\Upsilon_{\alpha}^{-1} \dot{\tilde{\Theta}}_{\alpha}\right)=0 \\
\tilde{\Theta}_{d}^{T} \Lambda^{T}\left(B_{p}^{T} P e_{a c} i^{T}+\Upsilon_{d}^{-1} \dot{\tilde{\Theta}}_{d}\right)=0
\end{array}
$$

By the differentiation of equations from 3.85a to 3.85d and from the knowledge attained in Eqn.s 3.100a, 3.100b, 3.100c and 3.100d, $\tilde{\Theta}$ terms can be determined. Assuming that the ideal terms, $\Theta^{*}$, remain constant, tilde terms turn out to be 
the same with the terms that have no tilde. Then the parameter update laws can be attained as in Eqn.s 3.101a, 3.101b, 3.101c and 3.101d,

$$
\begin{aligned}
& \dot{\Theta}_{x}=-\Upsilon_{x} B_{p}^{T} P e_{a c} x_{p}^{T} \\
& \dot{\Theta}_{r}=-\Upsilon_{r} B_{p}^{T} P e_{a c} r^{T} \\
& \dot{\Theta}_{\alpha}=-\Upsilon_{\alpha} B_{p}^{T} P e_{a c} f^{T} \\
& \dot{\Theta}_{d}=-\Upsilon_{d} B_{p}^{T} P e_{a c} i^{T}
\end{aligned}
$$

With the proper selection of positive definite adaptation rate matrices, $\Upsilon_{\alpha}, x, d, r$, as well as $P$ and $Q$ discussed in this Lyapunov analysis, the condition $\dot{V} \leq 0$ and thus the stability can be achieved. Nonetheless, the discussion about the convergence of parameters to the ideal cases or the asymptotic stability is still incomplete. Similar to the case discussed so far, if the derivative of Lyapunov function is negative semi-definite, the convergence of error in adaptive control of time-varying systems may be determined from Barbalat's Lemma and the following corollary [141.

Lemma 1. Let $\exists$ a uniformly continuous function $f(t): R^{+} \rightarrow R \forall t$. If $\exists$ $\lim _{t \rightarrow \infty} \int_{0}^{\infty}|f(\tau)| \mathrm{d} \tau$ and this limit of the integral is finite, then $\lim _{t \rightarrow \infty} f(t)=0$. Corollary 1. If $\exists$ square integrable $g$ such that $g(t) \in \mathrm{L}^{2} \cap \mathrm{L}^{\infty}$ and $\dot{g}$ is bounded, then $\lim _{t \rightarrow \infty} g(t)=0$.

Before using this corollary, remember that $\dot{V} \leq 0$ so far and the aim is to show $\lim _{t \rightarrow \infty} e_{a c}(t)=0$. First of all, since a Lyapunov function with $\dot{V} \leq 0$ satisfies $\dot{t} \leq V(0)<\infty$, it may be claimed that this Lyapunov function, $V$, is bounded. Besides, since the control error and adaptation parameters are arguments of $V$, they are also bounded. The stability of the reference model and the boundedness on the control input, the control error and the reference state, $x_{m}$ cause $x_{p}$ to remain bounded. That is, $e_{a c}, x_{p}, \Theta \in \mathrm{L}_{\infty}$. The sole point left in the before applying the Lemma of Barbalat is to show $e_{a c} \in \mathrm{L}^{2}$. By the fundamental knowledge;

$$
\int_{0}^{t} \dot{V}(\tau) \mathrm{d} \tau=V(t)-V(0)
$$

Since $\mathrm{V}$ is non-increasing and positive definite, $V(0)-V(t) \leq V(0)$. Then,

$$
-\int_{0}^{t} \dot{V}(\tau) \mathrm{d} \tau \leq V(0)<\infty
$$


Reminding $\dot{V}=-\frac{1}{2} e_{a c}^{T} Q e_{a c}$ and inserting into 3.103 .

$$
0 \leq \int_{0}^{t} \frac{1}{2} e_{a c}(\tau)^{T} Q e_{a c}(\tau) \mathrm{d} \tau<\infty
$$

Expression 3.104 shows $e_{a c} \in L_{2}$. Finally, since $\dot{e}_{c} \in L_{\infty}$, the pre-conditions to apply the Barbalat's Lemma are satisfied and $\lim _{t \rightarrow \infty} e_{a c}(t)=0$.

\subsubsection{Attitude Loop within MRAC Structure}

The attitude control loop in the MRAC structure still depends on the non-linear dynamic inversion. Since the angular momentum dynamics and the uncertain parameters are excluded, this loop only includes trigonometric relations. First of all, the reference model used in the attitude loop of MRAC is exactly the same as the reference model already explained. However, only the first derivative of the output is taken into consideration since the adaptive rate controller needs the first rates of the output as input. Now, defining pseudo controls for this loop, Eqn. 3.105 can be asserted.

$$
\nu=\left[\begin{array}{lll}
\dot{\Phi}_{p c} & \dot{\Theta}_{p c} & \dot{\Psi}_{p c}
\end{array}\right]^{T}
$$

Similar to the construction of the non-linear dynamic inversion scheme, the error dynamics should also be considered. The error can now be defined as the difference between the first derivatives of the outputs $\dot{y}$ and the reference model output rates, $\nu$. Since the deviation between reference command and system output is most possible, the stabilization of the error dynamics of first order should be considered. A stable dynamic can be achieved by a PI-like error controller. The error controller for this loop is as follows.

$$
\begin{gathered}
e=\dot{y}_{r e f}-\nu \\
\nu=\dot{y}_{r e f}+k_{p} e+k_{i} e / s
\end{gathered}
$$

Also note that the inversion of the relation in Eqn. 2.26 between the body rates and the Euler rates should be reminded. Then, these body rate commands in Eqn. 3.108 are inputs to the adaptation loop.

$$
\left[\begin{array}{l}
p_{c} \\
q_{c} \\
r_{c}
\end{array}\right]=\left[\begin{array}{ccc}
1 & 0 & -\sin (\theta) \\
0 & \cos (\phi) & -\sin (\phi) \cos (\theta) \\
0 & -\sin (\phi) & \cos (\phi) \cos (\theta)
\end{array}\right]\left[\begin{array}{l}
\dot{\phi}_{p c} \\
\dot{\theta}_{p c} \\
\dot{\psi}_{p c}
\end{array}\right]
$$


Finally, the pseudo control hedging is implemented by adding the hedge term to the first derivative of the output signal.The outer loop perceives the inner loop as an actuator. By slowing down the reference model, this method hides the actuator dynamics from error dynamics again and the reference model is restricted not to produce any command that the inner loop cannot follow. Through this, the actuator saturations, the integral wind-ups and the unlimited increase in adaptation gains are prevented. The first derivative of the system output is now on the scope and the equations for Pseudo-Control Hedging change as follows:

$$
\begin{gathered}
\dot{y}=\hat{v}+\Delta=\hat{\dot{y}}+\Delta \\
\hat{v}=\dot{y}=-\Delta \\
v_{p c h}=v-\hat{v} \\
v=\hat{v}+v_{p c h}
\end{gathered}
$$

Inserting Eqn.s 3.111 and 3.112 into Eqn. 3.109 .

$$
\begin{gathered}
d_{i}=v-\dot{y}=\hat{v}+v_{p c h}-\hat{v}-\Delta \\
\dot{y}=v-\hat{v}-v_{p c h}+\hat{v}+\Delta \\
\dot{y}=v+\Delta-v_{p c h}
\end{gathered}
$$

Since the reference input signal is assumed to be equal to the first derivative of the output, the modification in Eqn. 3.115 can now be applied to the reference model as follows:

$$
\begin{gathered}
\dot{y}_{r e f}=v_{r e f}-v_{p c h} \\
\ddot{y}_{r e f}=\omega_{0}^{2}\left(y_{c m d}-y_{r e f}\right)-2 \omega_{0} \zeta\left(\dot{y}_{r e f}-v_{p c h}\right)
\end{gathered}
$$

The modified pseudo control output from the error controller is described in Eqn 3.118 for an arbitrary axis:

$$
v_{i}=v_{i, r e f}+k_{p} e+k_{i} e / s-v_{p c h}
$$

Figure 3.11 summarizes the reference model structure in adaptive control using $\mathrm{PCH}$. 


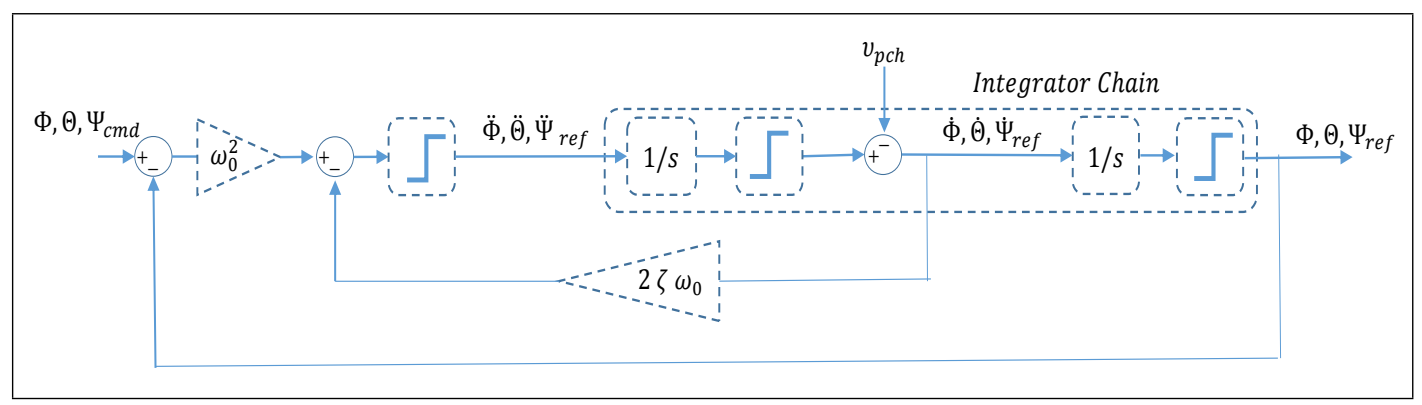

Figure 3.11: Reference Model Structure with Pseudo-Control Hedging

\subsection{Robustness Modifications to MRAC}

As a robustness improvement to MRAC, many approaches have been proposed and are present in literature. Narendra [141] suggests a persistent excitation in the input that has influence on all modes of the system. Such an approach aims at robustness against uncertainties and disturbances through the achievement of convergence to ideal parameters and the reduction of parameter drift. Other approaches mainly depend on modifications to the adaptive control law. Before any discussion about these methods, the Lyapunov analysis for unmatched uncertainties is most appropriate at this point.

\subsubsection{Lyapunov Analysis for Unmatched Uncertainties}

Let $\exists$ a non-linear term $\epsilon \in R^{n}$ representing the uncertainties. Extend Eqn.3.69 and Eqn. 3.86 with this term.

$$
\begin{gathered}
\dot{x}_{p}=A_{p} x_{p}+B_{p} \Lambda u+\alpha_{p} f\left(x_{p}\right)+d+\epsilon\left(x_{p}\right) \\
\dot{e}_{a c}=A_{m} e_{a c}+B_{p} \Lambda \tilde{\Theta}_{x} x_{p}+B_{p} \Lambda \tilde{\Theta}_{r} r+B_{p} \Lambda \tilde{\Theta}_{\alpha} f\left(x_{p}\right)+B_{p} \Lambda \tilde{\Theta}_{d} i+\epsilon\left(x_{p}\right)
\end{gathered}
$$

Utilizing the same Lyapunov function in Eqn 3.88 , the time derivative, $\dot{V}$ of the modified dynamics can be expressed as in Eqn.3.121

$$
\begin{aligned}
\dot{V}=\frac{1}{2}\left[\dot{e}_{a c}{ }^{T} P e_{a c}+e_{a c}^{T} P e_{a c}^{\cdot}\right] & +\operatorname{Tr}\left[\tilde{\Theta}_{x}^{T} \Upsilon_{x}^{-1} \dot{\tilde{\Theta}}|\Lambda|\right]+\operatorname{Tr}\left[\tilde{\Theta}_{r}^{T} \Upsilon_{r}^{-1} \dot{\tilde{\Theta}}_{r}|\Lambda|\right] \\
& +\operatorname{Tr}\left[\tilde{\Theta}_{\alpha}^{T} \Upsilon_{\alpha}^{-1} \dot{\tilde{\Theta}}_{\alpha}|\Lambda|\right]+\operatorname{Tr}\left[\tilde{\Theta}_{d}^{T} \Upsilon_{d}^{-1} \dot{\tilde{\Theta}}_{d}|\Lambda|\right]
\end{aligned}
$$




$$
\begin{array}{r}
\dot{V}=\frac{1}{2}\left[e_{a c}^{T} P A_{m} e_{a c}+e_{a c}^{T} A_{m}^{T} P e_{a c}+\epsilon\left(x_{p}\right) P e_{a c}\right]+e_{a c}^{T} P \epsilon\left(x_{p}\right)^{T}+e_{a c}^{T} P B_{p} \Lambda \tilde{\Theta}_{x} x_{p} \\
+x_{p}^{T} \tilde{\Theta}_{x}^{T} \Lambda^{T} B_{p}^{T} P e_{a c}+e_{a c}^{T} P B_{p} \Lambda \tilde{\Theta}_{r} r+r^{T} \tilde{\Theta}_{r}^{T} \Lambda^{T} B_{p}^{T} P e_{a c}+e_{a c}^{T} P B_{p} \Lambda \tilde{\Theta}_{\alpha} f\left(x_{p}\right) \\
\quad+f\left(x_{p}\right)^{T} \tilde{\Theta}_{\alpha}^{T} \Lambda^{T} B_{p}^{T} P e_{a c}+e_{a c}^{T} P B_{p} \Lambda \tilde{\Theta}_{d} i+i^{T} \tilde{\Theta}_{d}^{T} \Lambda^{T} B_{p}^{T} P e_{a c}
\end{array}
$$

Inserting Eqn. 3.96 into Eqn. 3.122 ,

$$
\begin{aligned}
& \dot{V}=- \frac{1}{2} e_{a c}^{T} Q e_{a c}+e_{a c}^{T} P \epsilon\left(x_{p}\right)+\operatorname{Tr}\left[\tilde{\Theta}_{x}^{T} \Lambda^{T}\left(B_{p}^{T} P e_{a c} x_{p}^{T}+\Upsilon_{x}^{-1} \dot{\tilde{\Theta}}_{x}\right)\right] \\
&+\operatorname{Tr}\left[\tilde{\Theta}_{r}^{T} \Lambda^{T}\left(B_{p}^{T} P e_{a c} r^{T}+\Upsilon_{r}^{-1} \dot{\tilde{\Theta}}_{r}\right)\right]+\operatorname{Tr}\left[\tilde{\Theta}_{\alpha}^{T} \Lambda^{T}\left(B_{p}^{T} P e_{a c} f^{T}+\Upsilon_{\alpha}^{-1} \dot{\tilde{\Theta}}_{\alpha}\right)\right] \\
&+\operatorname{Tr}\left[\tilde{\Theta}_{d}^{T} \Lambda^{T}\left(B_{p}^{T} P e_{a c} i^{T}+\Upsilon_{d}^{-1} \dot{\tilde{\Theta}}_{d}\right)\right]
\end{aligned}
$$

By equating all trace elements to zero as shown in Eqn.s 3.100a, 3.100b, 3.100 and $3.100 \mathrm{~d}$ with adaptive laws, the term $-\frac{1}{2} e_{a c}^{T} Q e_{a c}-e_{a c}^{T} P \epsilon\left(x_{p}\right) e_{a c}$ is left for Lyapunov analysis. Although $Q$ is still assumed to be positive definite and symmetric, the negative semi-definiteness of the whole expression is valid in a region which should be estimated.

$$
\begin{gathered}
\dot{V}=-\frac{1}{2} e_{a c}^{T} Q e_{a c}+e_{a c}^{T} P \epsilon\left(x_{p}\right) \leq 0 \\
-\frac{1}{2} e_{a c}^{T} Q e_{a c}+e_{a c}^{T} P \epsilon\left(x_{p}\right)=e_{a c}^{T}\left(-\frac{1}{2} Q e_{a c}+P \epsilon\left(x_{p}\right)\right) \leq 0 \\
-\frac{1}{2} Q e_{a c}+P \epsilon\left(x_{p}\right) \leq 0
\end{gathered}
$$

Taking norm of both sides,

$$
\begin{array}{r}
\left\|-\frac{1}{2} Q e_{a c}+P \epsilon\left(x_{p}\right)\right\| \leq 0 \\
-\frac{1}{2} \operatorname{eig}(Q)\left\|e_{a c}\right\|+\operatorname{eig}(P)\left\|\epsilon\left(x_{p}\right)\right\| \leq 0
\end{array}
$$

Rearranging Eqn $3.127 \mathrm{~b}$,

$$
\left\|e_{a c}\right\| \geq \frac{2 \cdot \operatorname{eig}(P)\left\|\epsilon_{a c}\right\|}{\operatorname{eig}(Q)}
$$

To find the maximum upper bound under which the derivative of Lyapunov function will no longer be negative semi definite(i.e. it will be positive definite), the maximum value the right side of the expression in Eqn. 3.128 could take should be considered. This is possible only if the maximum eigenvalue of $P$ and the minimum eigenvalue of $Q$ are taken into account as well as the existence of an upper bound to the unmatched uncertainties. Then Eqn. 3.128 becomes:

$$
\left\|e_{a c}\right\| \geq \frac{2 \cdot \operatorname{eig}(P)_{\max } \epsilon_{a c, \max }}{e i g(Q)_{\min }} \geq e_{\max }
$$


As a result of these unmatched uncertainties and the existence of such an upper bound, $e_{\max }$, the adaptive parameters of the system may drift when below the boundary, causing drastic increase in parameters and commands which together trigger instability.

\subsubsection{Dead-Zone Modification}

Dead zone modification uses the idea of turning off the adaptation when the error goes below the defined bound $e_{\max }$ in Eqn. 3.129. The difficulty of this approach is the determination of the upper bound as well as the necessity of persistent excitation to eliminate the parameter drift.

\subsubsection{Sigma-Modification}

The addition of a pure damping term to the parameter update law is known as $\sigma$ modification. The adaptive laws are as follows with the addition of error element:

$$
\begin{aligned}
& \dot{\Theta}_{x}=-\Upsilon_{x}\left[B_{p}^{T} P e_{a c} x_{p}^{T}+\sigma \Theta_{x}\right] \\
& \dot{\Theta}_{r}=-\Upsilon_{r}\left[B_{p}^{T} P e_{a c} r^{T}+\sigma \Theta_{r}\right] \\
& \dot{\Theta}_{\alpha}=-\Upsilon_{\alpha}\left[B_{p}^{T} P e_{a c} f^{T}+\sigma \Theta_{\alpha}\right] \\
& \dot{\Theta}_{d}=-\Upsilon_{d}\left[B_{p}^{T} P e_{a c} i^{T}+\sigma \Theta_{d}\right]
\end{aligned}
$$

The trace elements in $\dot{V}$ are now changed. Inserting 3.130a, 3.130b, 3.130c and $3.130 \mathrm{~d}$ into 3.123 .

$$
\dot{V}=-\frac{1}{2} e_{a c}^{T} Q e_{a c}+e_{a c}^{T} P \epsilon\left(x_{p}\right)-\sigma \operatorname{Tr}\left[\tilde{\Theta}_{x}^{T} \tilde{\Theta}_{x}+\tilde{\Theta}_{r}^{T} \tilde{\Theta}_{r}+\tilde{\Theta}_{\alpha}^{T} \tilde{\Theta}_{\alpha}+\tilde{\Theta}_{d}^{T} \tilde{\Theta}_{d}\right]
$$

For any $\tilde{\Theta}_{i}$, it may be asserted that $\exists$ positive constants $k$ and $c_{i}$ such that $\dot{V}<0$ outside a compact set $\mathrm{D},\left\|e_{a c}\right\| \leq k$ and $\left\|\operatorname{vec}\left(\tilde{\Theta}_{i}^{T}\right)\right\| \leq c_{i}$. According to [141, $e_{a c}$ and $\tilde{\Theta}_{i}$ converge to this set and remain bounded. This bound can be adjusted by the proper selection of $\sigma$. Some of the problems caused by unmatched uncertainties remain here due to the additional term " $\sigma \tilde{\Theta} "$. As the error stays close to zero, the elements of adaptation matrices are also drawn 
back to zero. Therefore, this term is named as the forgetting term. A way to overcome this is again a switching scheme or another modification.

\subsection{4 e-Modification}

The extension of the adaptation with a variable damping term scaled by the norm of the training error is known as e-modification. The modification in adaptive laws are as follows with the addition of $\sigma$ element.

$$
\begin{aligned}
& \dot{\Theta}_{x}=-\Upsilon_{x}\left[B_{p}^{T} P e_{a c} x_{p}^{T}+\left\|e_{a c}\right\| \sigma \Theta_{x}\right] \\
& \dot{\Theta}_{r}=-\Upsilon_{r}\left[B_{p}^{T} P e_{a c} r^{T}+\left\|e_{a c}\right\| \sigma \Theta_{r}\right] \\
& \dot{\Theta}_{\alpha}=-\Upsilon_{\alpha}\left[B_{p}^{T} P e_{a c} f^{T}+\left\|e_{a c}\right\| \sigma \Theta_{\alpha}\right] \\
& \dot{\Theta}_{d}=-\Upsilon_{d}\left[B_{p}^{T} P e_{a c} i^{T}+\left\|e_{a c}\right\| \sigma \Theta_{d}\right]
\end{aligned}
$$

The modified $\dot{V}$ is then as follows:

$$
\dot{V}=-\frac{1}{2} e_{a c}^{T} Q e_{a c}+e_{a c}^{T} P \epsilon\left(x_{p}\right)-\sigma\left\|e_{a c}\right\| \operatorname{Tr}\left[\tilde{\Theta}_{x}^{T} \tilde{\Theta}_{x}+\tilde{\Theta}_{r}^{T} \tilde{\Theta}_{r}+\tilde{\Theta}_{\alpha}^{T} \tilde{\Theta}_{\alpha}+\tilde{\Theta}_{d}^{T} \tilde{\Theta}_{d}\right]
$$

As in $\sigma$-modification, the deviations of adaptation parameters from the ideals are here bounded. The extended term is not a forgetting factor here since the term drops when error is zero. However, there still exists a problem with emodification. The excitation of the system in one axis causing the activation of e-modification results in the approaching of the adaptation parameters in other axes to zero. To prevent the convergence of adaptation to zero, e-modification can be separated in each control axis by multiplying the additional term with the magnitude of the control error in each axis. This new multiplier can be defined as follows:

$$
E_{a s}=\left[\begin{array}{ccc}
\left|e_{x}\right| & 0 & 0 \\
0 & \left|e_{y}\right| & 0 \\
0 & 0 & \left|e_{z}\right|
\end{array}\right]
$$


The adaptive laws can finally be obtained as:

$$
\begin{aligned}
& \dot{\Theta}_{x}=-\Upsilon_{x}\left[B_{p}^{T} P e_{a c} x_{p}^{T}+\left\|e_{a c}\right\| \sigma \Theta_{x} E_{a s}\right] \\
& \dot{\Theta}_{r}=-\Upsilon_{r}\left[B_{p}^{T} P e_{a c} r^{T}+\left\|e_{a c}\right\| \sigma \Theta_{r} E_{a s}\right] \\
& \dot{\Theta}_{\alpha}=-\Upsilon_{\alpha}\left[B_{p}^{T} P e_{a c} f^{T}+\left\|e_{a c}\right\| \sigma \Theta_{\alpha} E_{a s}\right] \\
& \dot{\Theta}_{d}=-\Upsilon_{d}\left[B_{p}^{T} P e_{a c} i^{T}+\left\|e_{a c}\right\| \sigma \Theta_{d} E_{a s}\right]
\end{aligned}
$$

\subsection{Integral Back-stepping}

First proposed by Kanellakopoulos [142] and put into application by Tan [143], integral back-stepping method is employed for quadrotors by Bouabdallah in [100] where asymptotic stability is guaranteed and some robustness to uncertainties is achieved. Moreover, the integral action deals with the steady state error accumulation. The control strategy can be summarized as:

$$
\begin{gathered}
U_{\text {roll }}=\frac{1}{b_{1}}\left[\left(1-c_{1}^{2}+\lambda_{1}\right) e_{1}+\left(c_{1}+c_{2}\right) e_{2}-c_{1} \lambda_{1} \kappa_{1}+\ddot{\phi}_{d}-\dot{\theta} \dot{\psi} a_{1}-\dot{\theta} \Omega a_{2}\right] \\
U_{\text {pitch }}=\frac{1}{b_{2}}\left[\left(1-c_{3}^{2}+\lambda_{2}\right) e_{3}+\left(c_{3}+c_{4}\right) e_{4}-c_{3} \lambda_{2} \kappa_{2}+\ddot{\theta} d-\dot{\phi} \dot{\psi} a_{3}+\dot{\phi} \Omega a_{4}\right] \\
U_{\text {yaw }}=\frac{1}{b_{3}}\left[\left(1-c_{5}^{2}+\lambda_{3}\right) e_{5}+\left(c_{5}+c_{6}\right) e_{6}-c_{5} \lambda_{3} \kappa_{3}\right]
\end{gathered}
$$

where $a_{1}=\left(I_{y y}-I_{z z}\right) / I_{x x} ; a_{2}=J_{r} / I_{x x} ; b_{1}=1 / I_{x x} ; b_{2}=1 / I_{y y} ; b_{3}=1 / I_{z z} ; \Omega$ is propeller angular speed; $c_{2}$ is the angular speed loop convergence constant; $c_{3}, c_{4}, c_{5}, c_{6}, \lambda_{2}, \lambda_{3}$ are positive constants and $\kappa_{2}, \kappa_{3}$ are the integral track errors of other states. The necessity of gain adjustments for this method is clear and after a pre-work, the results in Figures 3.12 can be highlighted. Any increase in the values of $c_{1}$ variables brings about results such as smaller overshoot, less numbers of oscillations and shorter settling time. The delay and difference in the convergence of the sinusoidal responses to the sinusoidal input is clear in Figure $3.12(\mathrm{~d})$. However, the results achieved by the greatest $c_{1}$ value are still closest to the desired trajectory. The smallest value of $c_{2}$ achieves the smallest overshoot while the time to respond is open to discussion. Nevertheless, for the sinusoidal input case, the results of the highest values remains closer to the desired trajectory. for this analyses, all parameters except the investigated ones are kept constant. 

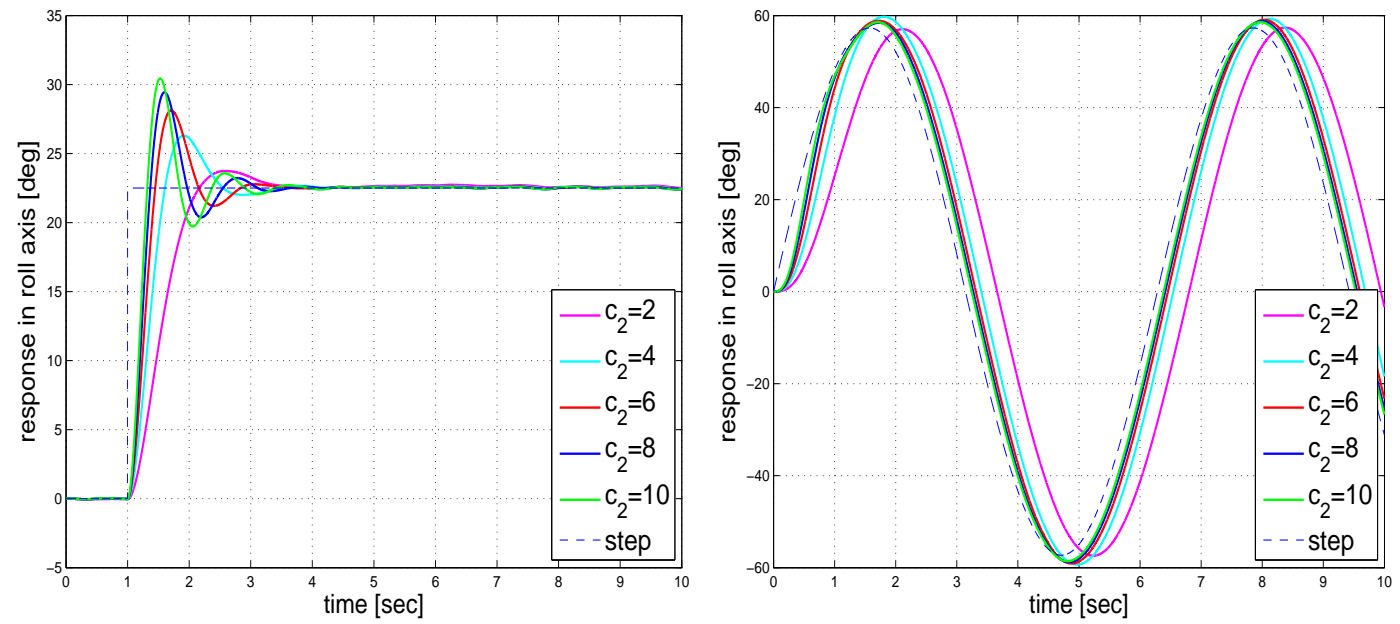

(a) Gain Adjustments for various $c_{2}$ values with (b) Gain Adjustments for various $c_{2}$ values with a step input a sine input
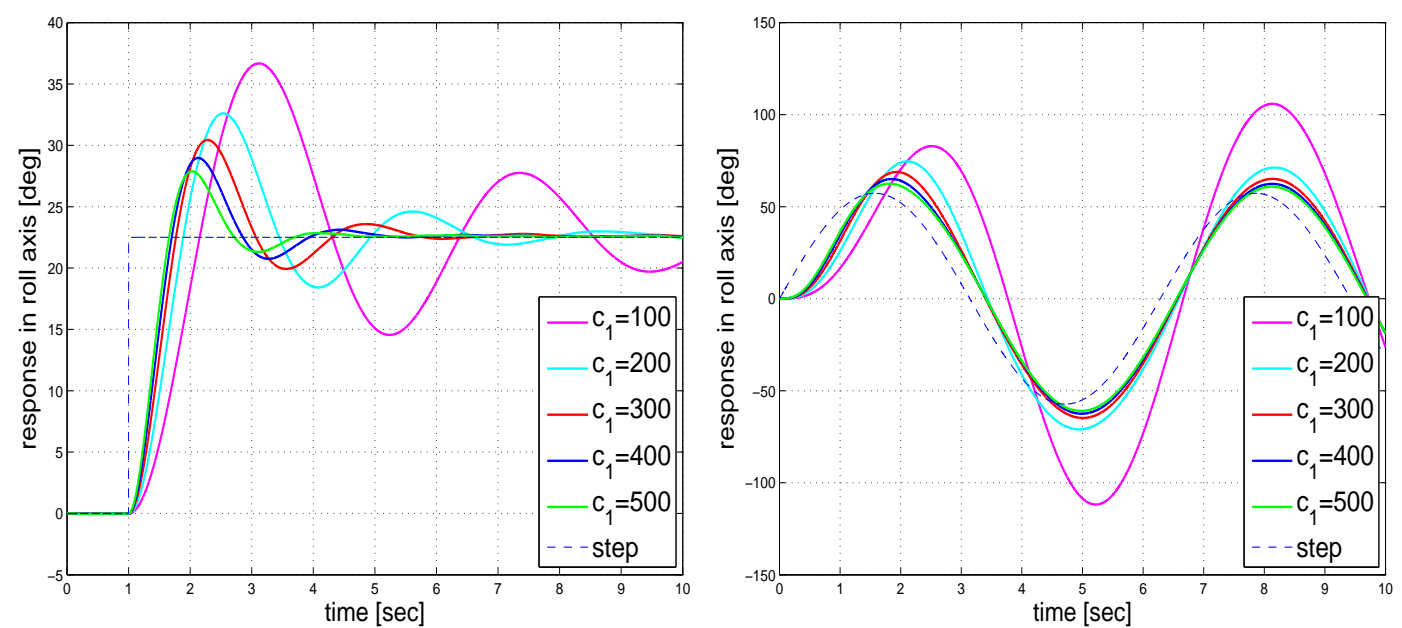

(c) Gain Adjustments for various $c_{1}$ values with a (d) Gain Adjustments for various $c_{1}$ values with step input

a sine input

Figure 3.12: Gain Adjustments for Integral Backstepping Method 


\section{CHAPTER 4}

\section{RESULTS AND DISCUSSION FOR VARIOUS CONTROLLER OPTIONS}

\subsection{Simulation Notes}

This section presents the results of the attitude control strategies explained in the previous chapter. The system is simulated with both noiseless and noisy measurements essential for reflecting the effect of real sensors. The parameter changes that represent the uncertainties in parameters and the presence of unknown disturbances are taken into consideration as well. The scenario of parameter uncertainty includes the insertion of the parameters of an entirely different quadrotor model into the simulation code. The unknown disturbance is modelled as a pulse wave element with certain period, width, amplitude and phase. In the procedure, such a pulse is added to the moment generated in the axis of desired motion. That is, for a desired rolling trajectory fed to the system, the rolling moment generation is extended with this element. The properties of the quadrocopter platform to be used in analysis can be found in Table 4.1 as given in [133].

Table 4.1: Parameters

\begin{tabular}{|c|c|c|}
\hline Parameter & Value & Unit \\
\hline$I_{x x, y y}$ & $5.6 \cdot 10^{-3}$ & $\mathrm{~kg} \cdot \mathrm{m}^{2}$ \\
\hline$I_{z z}$ & $8.1 \cdot 10^{-3}$ & $k g \cdot m^{2}$ \\
\hline mass & 0.48 & $\mathrm{~kg}$ \\
\hline
\end{tabular}


Another element that should be discussed for the simulations is the type of the command fed to the system. In the simulations of the attitude controller options, constant and circular trajectory tracking problems are taken into account. Therefore, a desired constant orientation in attitude and a sinusoidal attitude tracking command can initially be defined for simulations. The input types can be found on Table 4.2. Note that firstly both commands types are given only in one axis for different scenarios. Then, step commands that represent the desired trajectories in two axes are provided to discuss the capabilities of the controllers further. The input cases are present in Table 4.2 .

Table 4.2: Input Types

\begin{tabular}{|c|c|c|}
\hline Input Type & Command and Axes & Input Value [deg] \\
\hline Constant Trajec & Step Roll & 25 \\
\hline Constant Trajectory & Step Roll \& Pitch & {$[15,15]$} \\
\hline Circular Trajectory & Sinusoidal Roll & $A \sin (\omega t)$ \\
\hline
\end{tabular}

Noise modelling within the scope of this work is essential since the capabilities of any adaptation purpose strongly depend on the resilience of the controller against the presence of disturbances and noise. For noise modelling, the angular measurements are extended with the addition of discrete-time white Gaussian noise. The probability density function of such noise with n-dimension can be stated as in Eqn. 4.1. In this equation, $\mathrm{K}$ denotes the covariance matrix, $\mathrm{x}$ is the $\mathrm{n}$-length vector and $\mu$ is the mean value vector.

$$
f(x)=\left((2 \pi)^{n} \operatorname{det}(K)\right)^{-1 / 2} e^{-(x-\mu)^{T} K^{-1}(x-\mu) / 2}
$$

The level of distortion assumed in measurements can be summarized as statistically around $10^{-6} \mathrm{deg}$ for attitude angles and $10^{-2} \mathrm{deg} / \mathrm{s}$ for Euler and body angular rates. To show the effect of the uncertain parameters on the system and the controllers in the model, the moments of inertia in the system blocks are changed with the moments of inertia of another quadrotor model. To get a better comprehension about the scenarios, Table 4.3 can be visited. 
Table 4.3: Simulation Scenarios with their figure numbers

\begin{tabular}{|c|c|c|c|}
\hline Input \& Scenario & Step Roll & Sinusoidal Roll & Step Roll \& Pitch \\
\hline no noise & Fig. 4.1 & Fig. B.1 & Fig. A.1 \\
\hline noise & Fig. 4.2 & Fig. $\overline{\bar{B} .2}$ & Fig. A.2 \\
\hline uncertain parameter & Fig. 4.3 & Fig. B.3 & Fig. A.3 \\
\hline unknown disturbance & Fig. 4.4 & Fig. B.4 & Fig. $\overline{A .4}$ \\
\hline
\end{tabular}

\subsection{Simulation Results for Constant Attitude Tracking}

Figures 4.1, 4.2, 4.3 and 4.4 indicate responses and error accumulations in attitude angles for the fixed step input cases. The general trends in the simulation results of the constant attitude trajectory tracking highlight the necessity of the use of the adaptation concept. First of all, the responses in the roll states of all the methods track comparably well for noiseless case as depicted in Figures 4.1(a) and 4.1(b). In this case, the base controller built on Model Refence Control and Non-Linear Dynamic Inversion performs best while both Model Reference Adaptive and Integral Backstepping Controllers also perform very well with excusable exceptions. There exists a second delay in IB as well as overand under-shootings in both methods. The errors in pitch and yaw states could be kept acceptably close to zero for all the methods in noiseless case. The results of IB and base controller in the noisy case demonstrate a similar trend to the results in the noiseless case; however, for MRAC, the existence of a drift of several degrees from the target with time is obvious in Figures 4.2(a), In Figures 4.3(a) and 4.3(b), the roll response of the base control method fails while both Integral Backstepping and adaptive controllers achieve to converge to the desired trajectory. It is clear that the drift in MRAC scheme is still present. Above all, the vitality of the adaptation is clearly explicit with the results in the presence of unknown disturbance. Obviously visible in Figures 4.4(a) and 4.4(b), the only controller that achieves the desired trajectory is MRAC which could achieve to tolerate the pulses in the system in this case. 


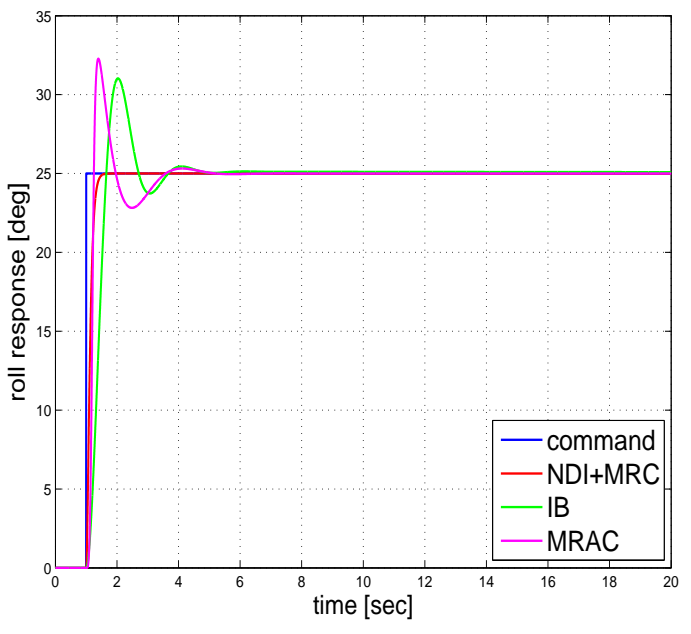

(a) Roll State Response

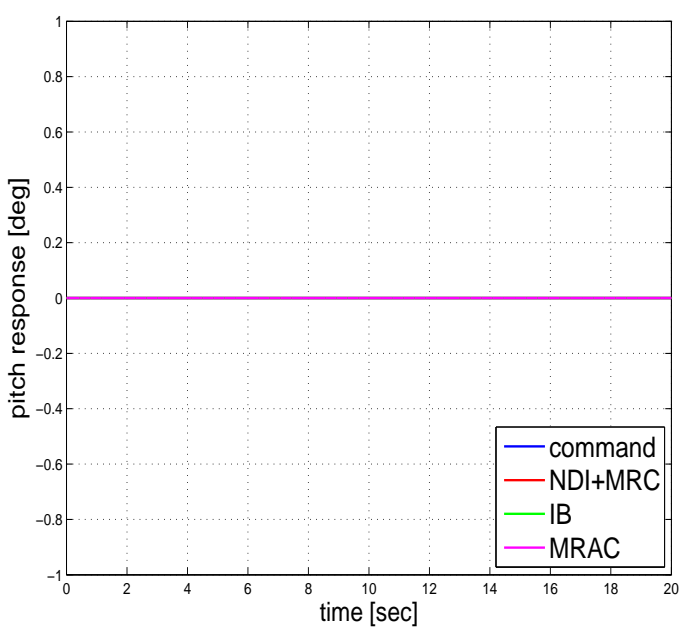

(c) Pitch State Response

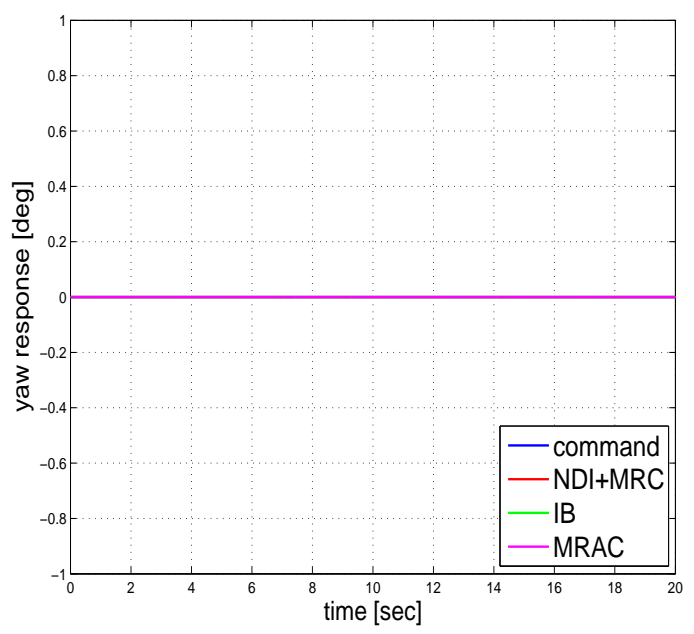

(e) Yaw State Response

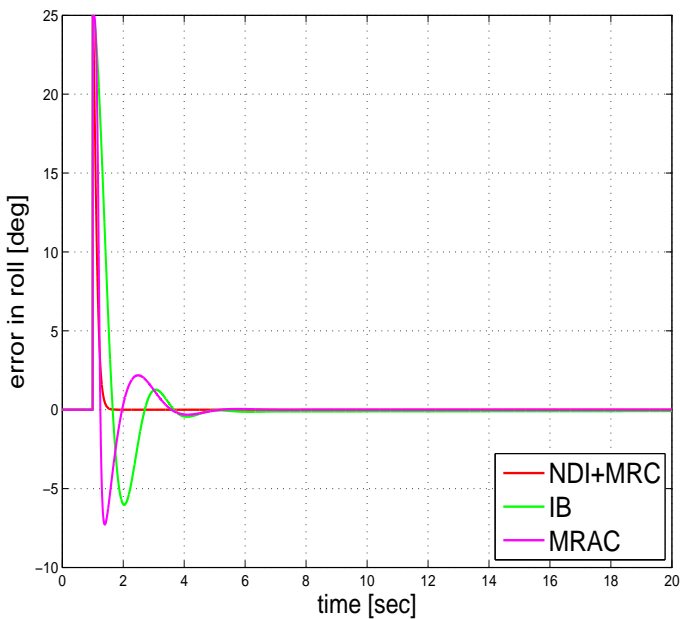

(b) Roll Error

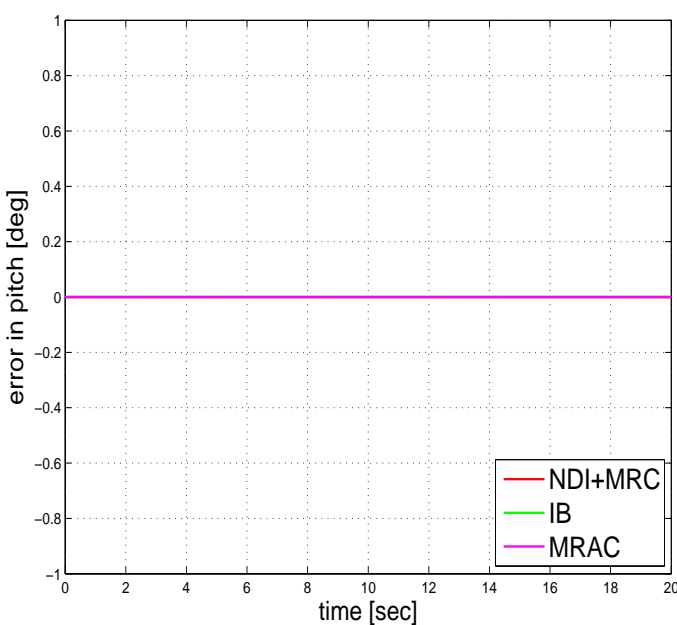

(d) Pitch Error

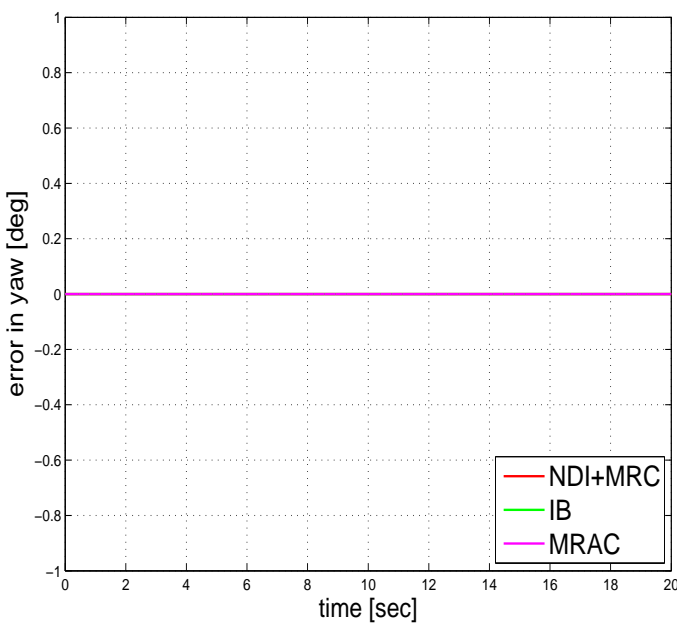

(f) Yaw Error

Figure 4.1: Simulation of fixed angular orientation control with no noise 


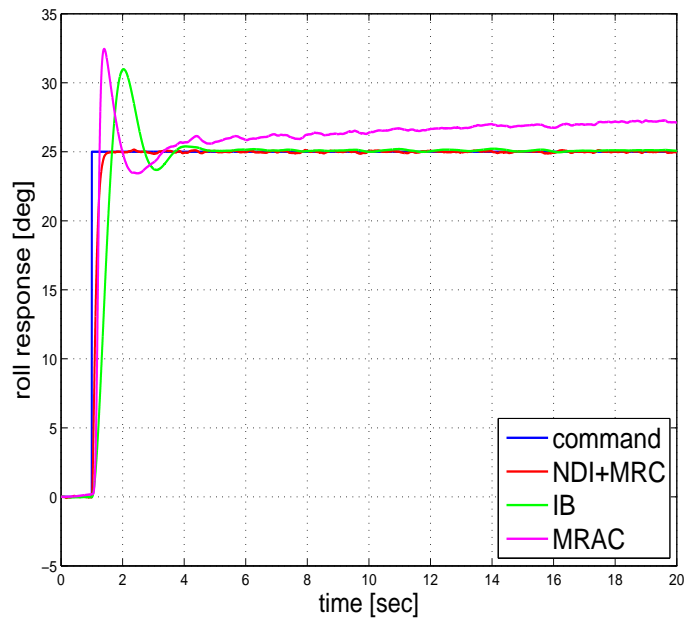

(a) Roll State Response

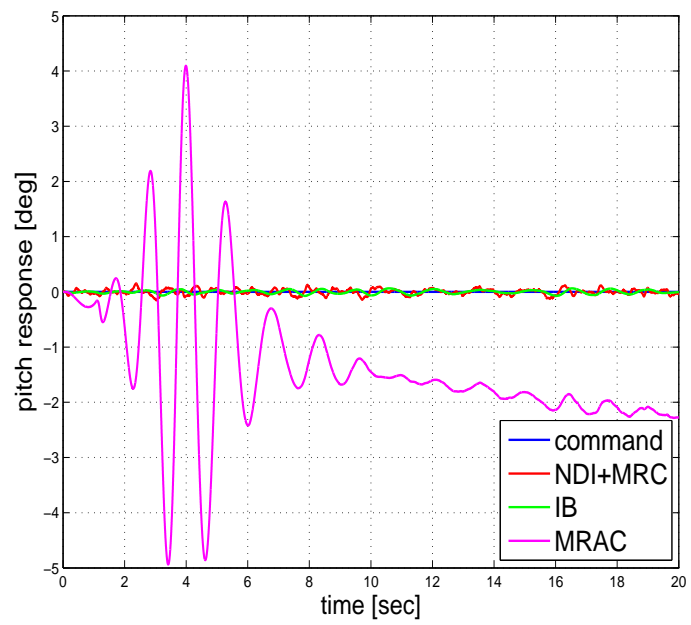

(c) Pitch State Response

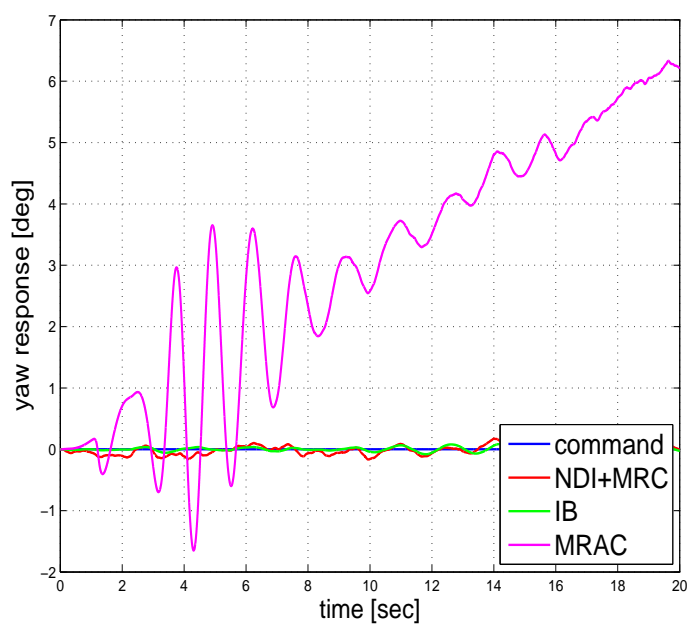

(e) Yaw State Response

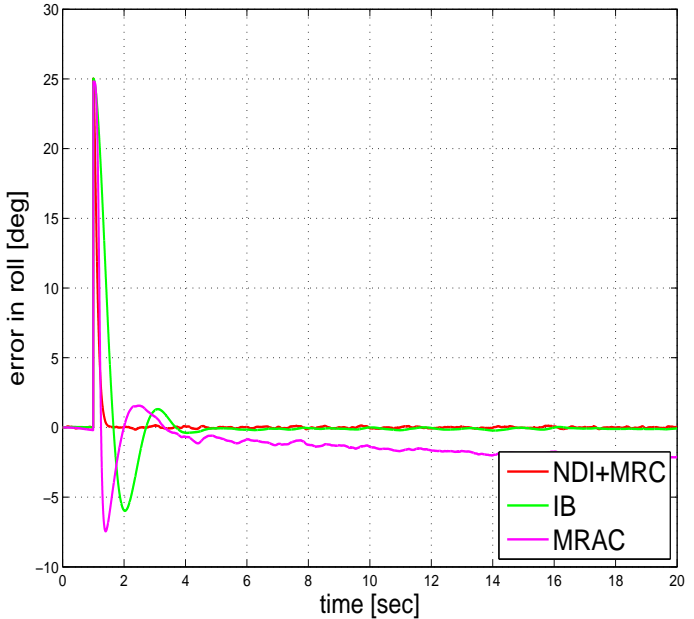

(b) Roll Error

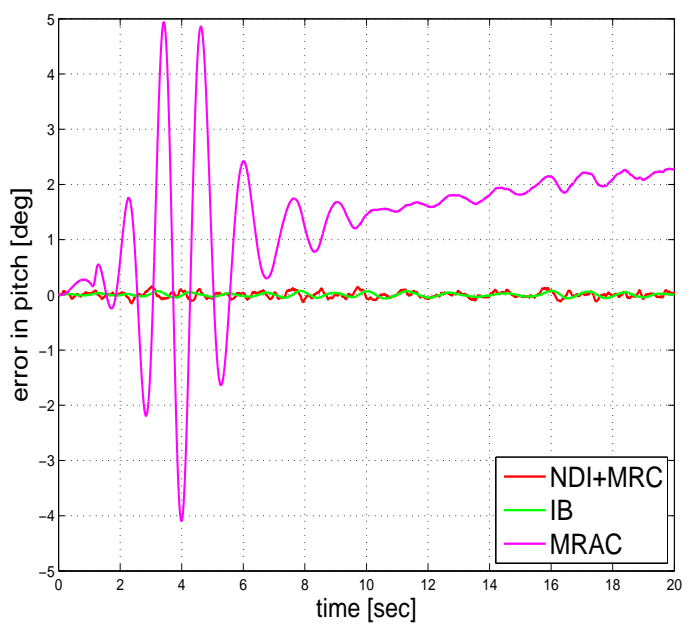

(d) Pitch Error

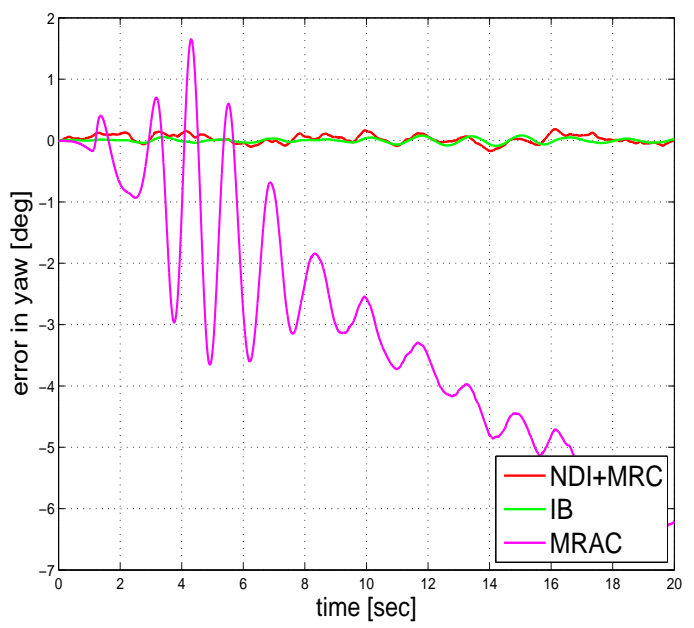

(f) Yaw Error

Figure 4.2: Simulation of fixed angular orientation control with noise addition 


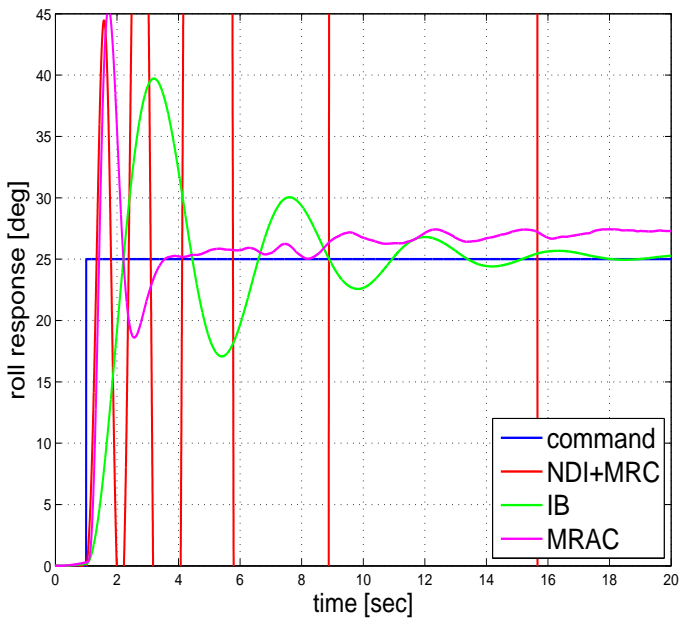

(a) Roll State Response

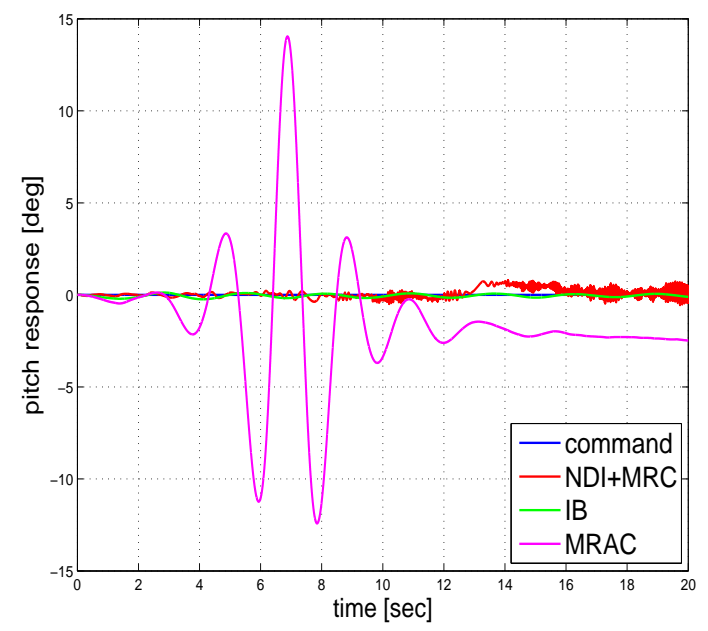

(c) Pitch State Response

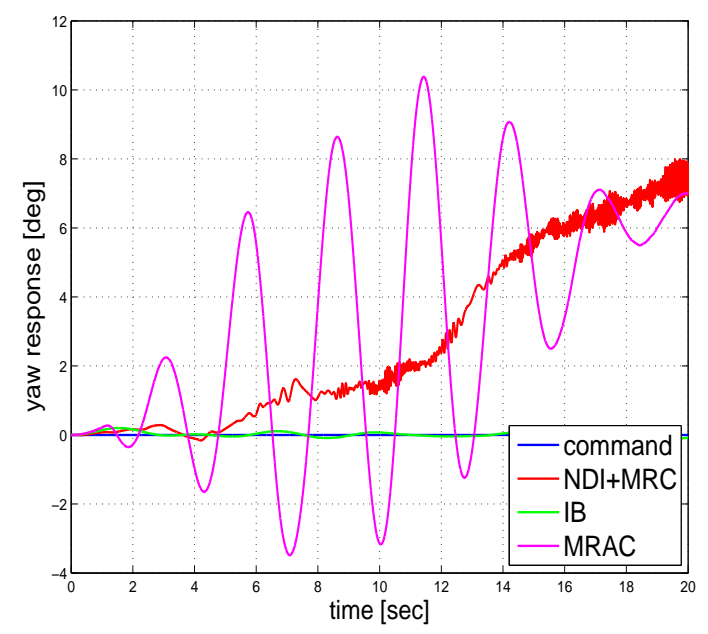

(e) Yaw State Response

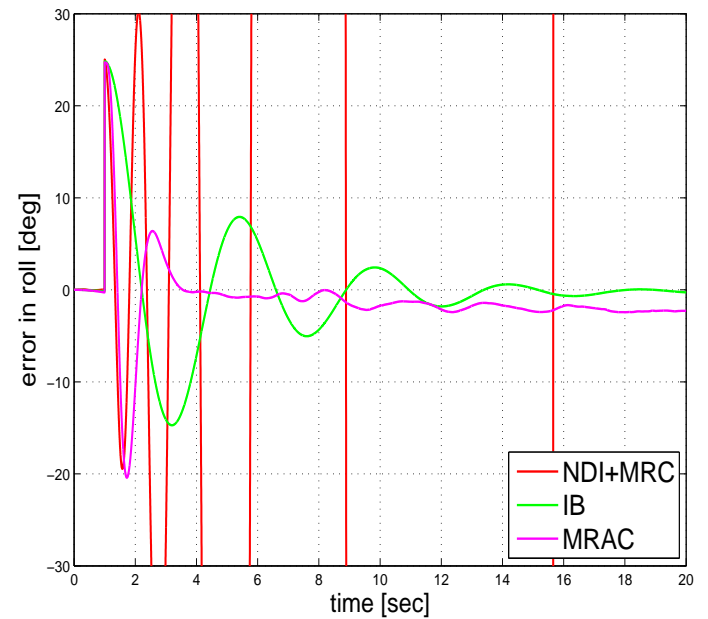

(b) Roll Error

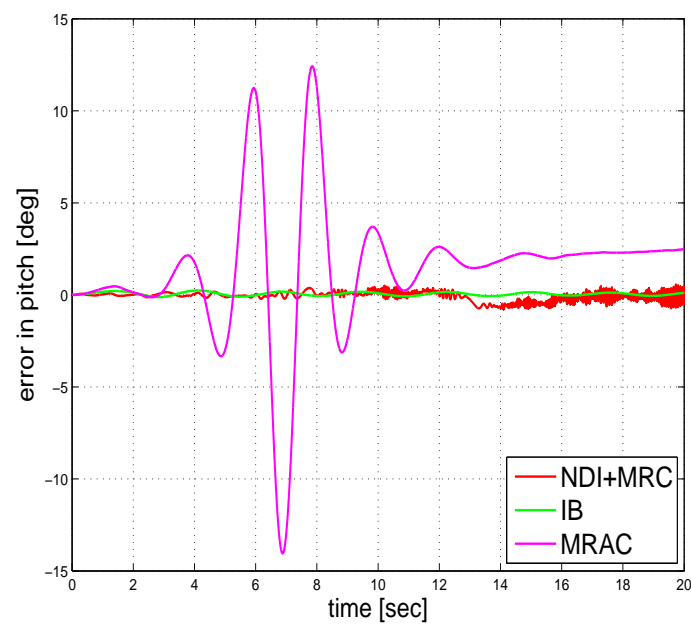

(d) Pitch Error

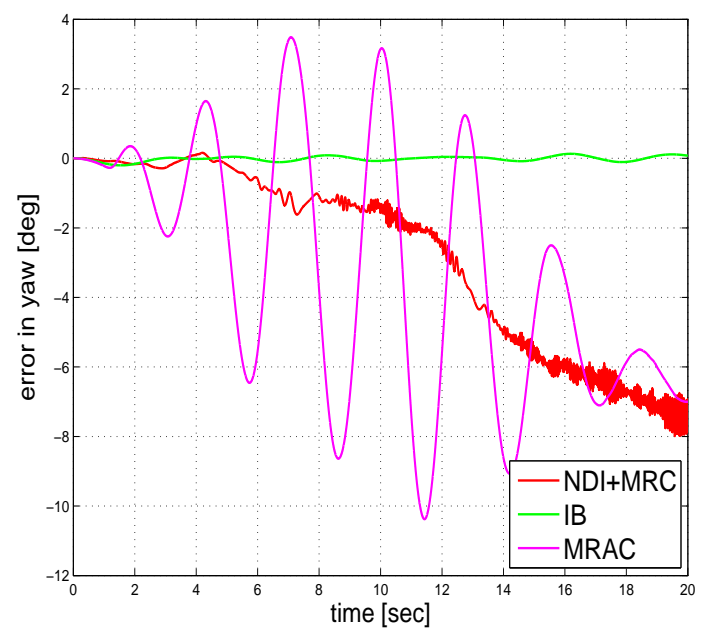

(f) Yaw Error

Figure 4.3: Simulation of fixed angular orientation control with a different quadrotor model 


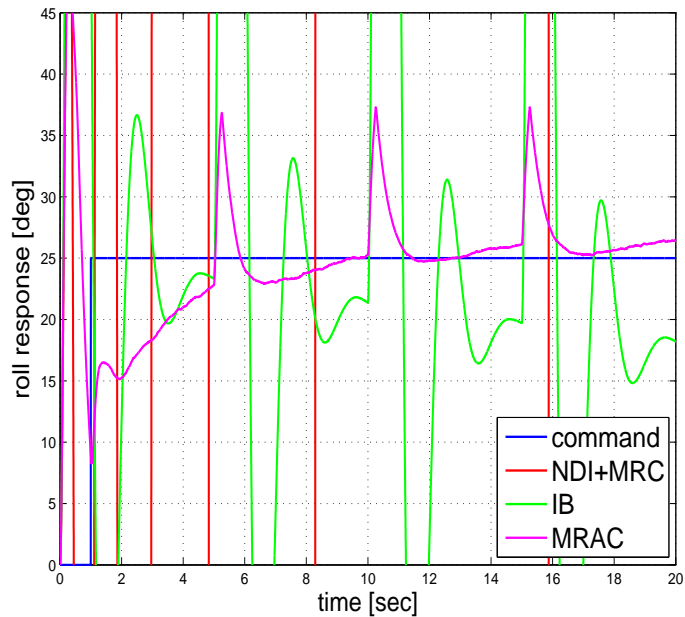

(a) Roll State Response

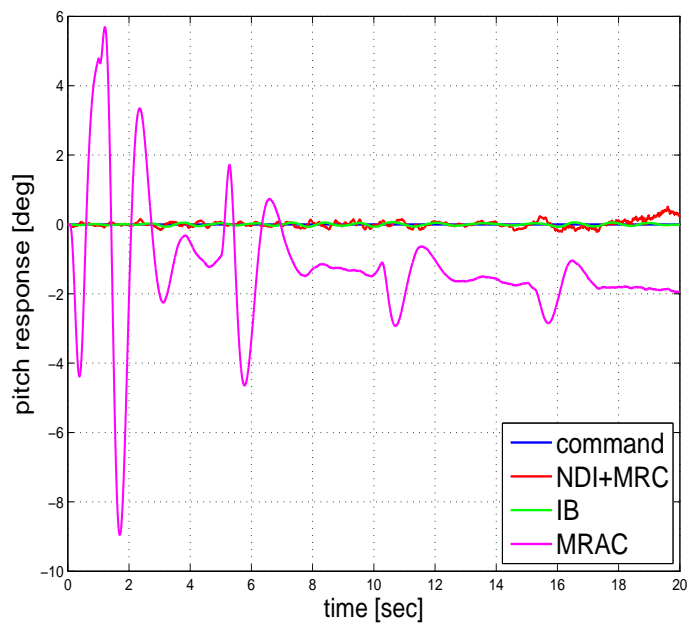

(c) Pitch State Response

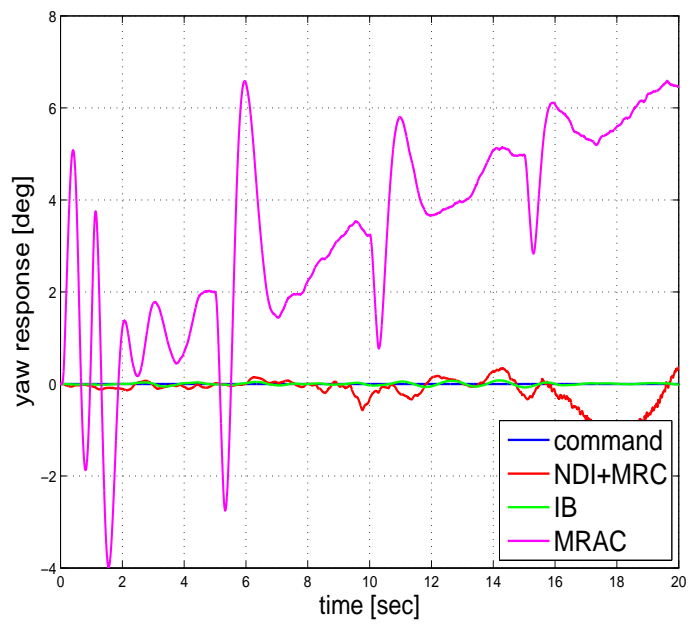

(e) Yaw State Response

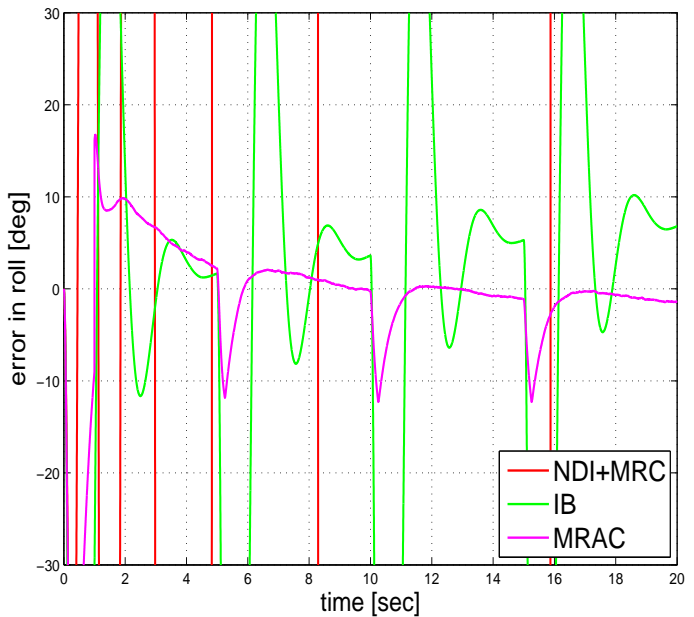

(b) Roll Error

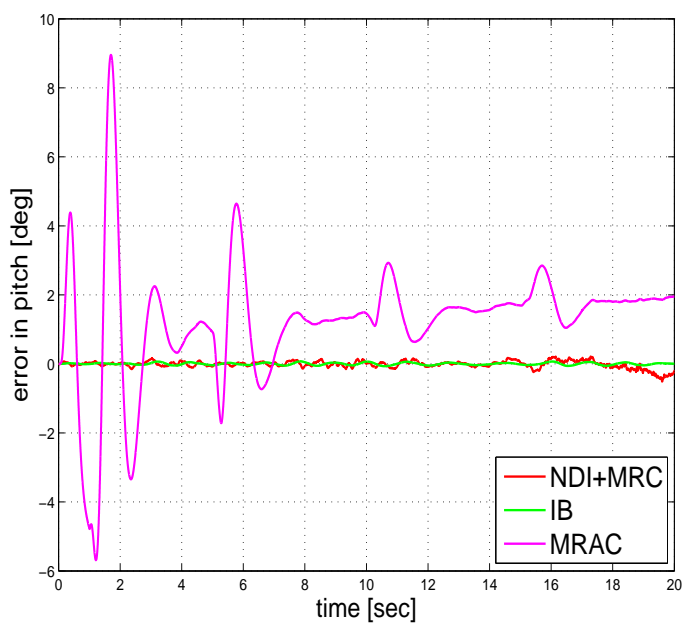

(d) Pitch Error

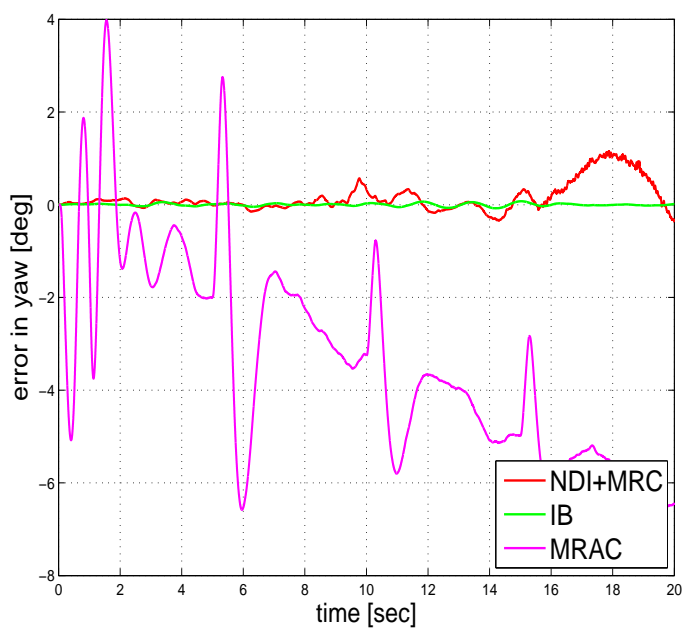

(f) Yaw Error

Figure 4.4: Simulation of fixed angular orientation control in the presence of unknown disturbance 


\subsection{Simulations Results for Sinusoidal Input with Various Periods}

The second fundamental input fed to the controller systems as a tracking challenge is the sinusoidal command type. The sinusoidal command can be represented by $A \sin (\omega t)$ where $A$ is amplitude and $\omega$ is the angular frequency. In order to investigate the influence of the adaptation and the other methods against the changes in the amplitude and frequency of the sinusoidal input, the scenarios in Table 4.4 can be considered. These scenarios include two different amplitude and frequency values which sum up to a total of 4 cases. Note that all these cases include parameter uncertainty.

Table 4.4: Figures regarding Simulations with Sinusoidal Inputs under Parameter Uncertainty organized according to their Amplitude and Periods

\begin{tabular}{|c|c|c|c|}
\hline Amplitude \& Period & \multicolumn{3}{|c|}{$\omega=1$} \\
\hline$A=1$ & Figures $4.5(\mathrm{a})$, & B.5(a), & B.6(a) \\
\hline \multirow{2}{*}{$A=0.5$} & Figures $4.5(\mathrm{c})$, & B.5(c), & B.6(c) \\
\hline & \multicolumn{3}{|c|}{$\omega=2$} \\
\hline$A=1$ & Figures $4.5(\mathrm{~b})$ & B.5(b), & B.6(b) \\
\hline$A=0.5$ & Figures $4.5(\mathrm{~d})$, & B.5(d), & B.6(d) \\
\hline
\end{tabular}

The results of these scenarios with sinusoidal commands are present in Figures 4.5. Figures B.1, B.2, B.3 and B.4 in Appendices can be visited for further results with sinusoidal commands. Concerning the results of the noiseless and noisy cases, it can be claimed that the error of Integral Backstepping-based Controller is greater than that of other methods as can be seen in Figures ?? and B.2(b). The gap between the desired trajectory and the trajectory that IB-controller could achieve even soars in the existence of parameter uncertainty. For the discussed cases, the performance of both MRAC and NLDI + MRC are within the desired limits. Besides, the performance of NLDI+MRC show smaller difference in phase than MRAC. Nevertheless, as in the cases with constant orientation trajectory tracking, the presence of unknown disturbance could not be tolerated by IB and NLDI + MRC techniques which diverge with the hit of the pulse waves. The significance of adaptation is undeniable again thanks to 
the performance of MRAC under the circumstance with unknown disturbance in the system. Although, MRAC does not fail, it should be stated that the phase difference between MRAC results and the desired trajectory is more obvious when compared to the previous cases. All these phenomena can be explored in Figures B.3 and B.4.

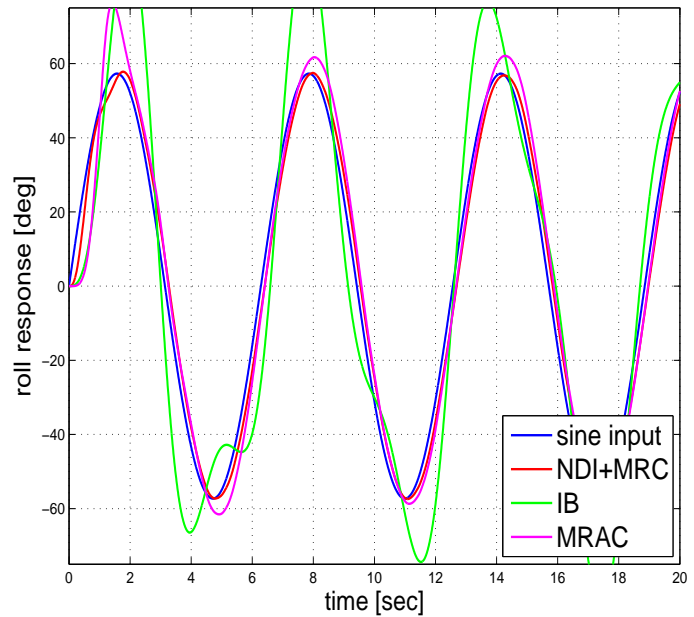

(a) Roll Response for $\mathrm{A}=1$ and omega $=1$

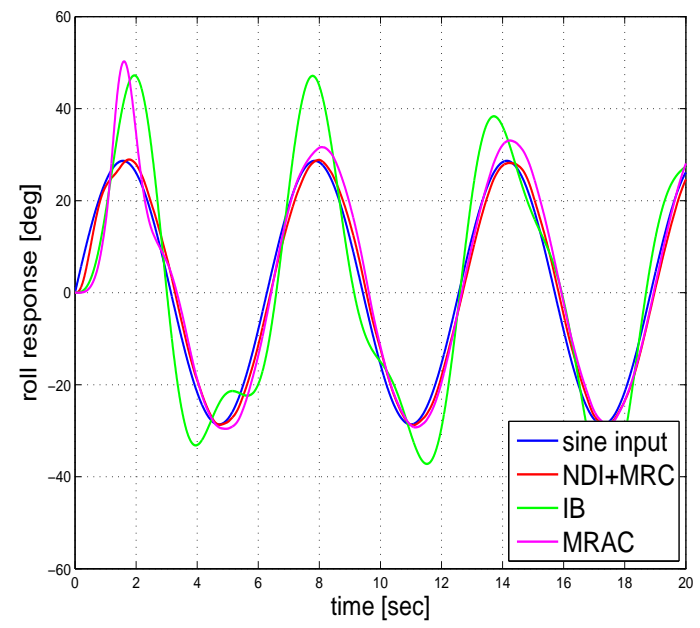

(c) Roll Response for $\mathrm{A}=0.5$ and omega $=1$

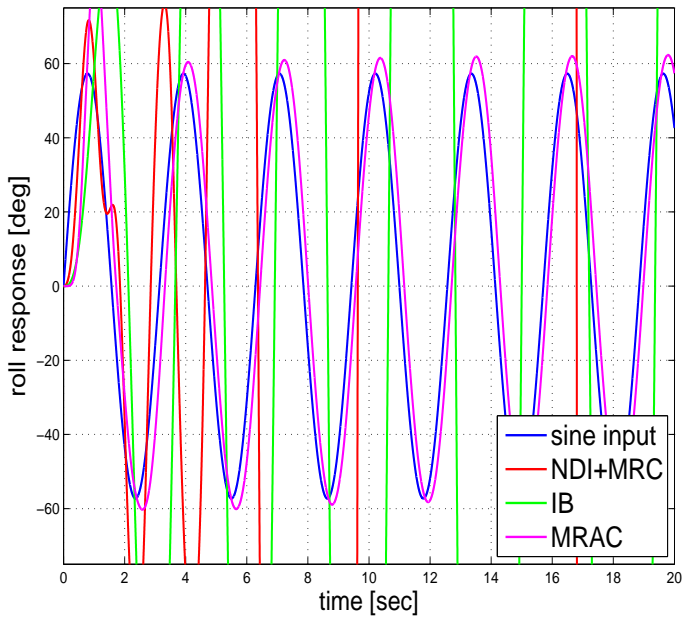

(b) Roll Response for $\mathrm{A}=1$ and omega $=2$

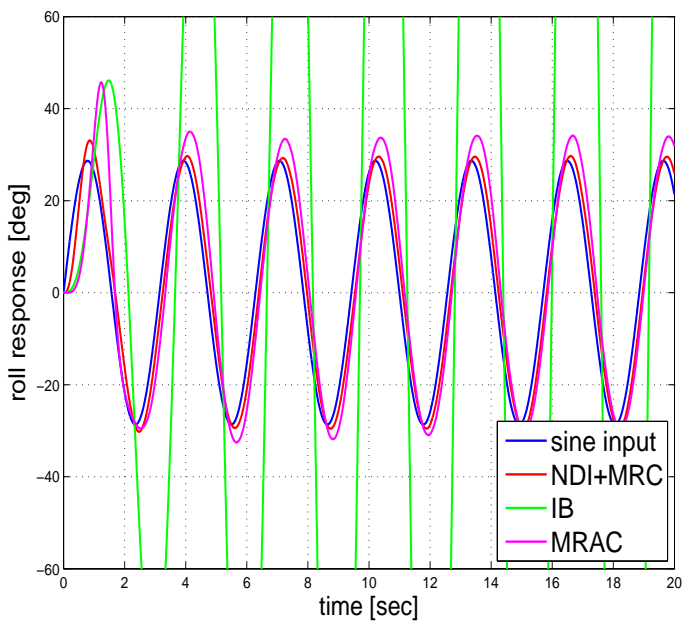

(d) Roll Response for $\mathrm{A}=0.5$ and omega $=2$

Figure 4.5: Simulation of sinusoidal command with various periods and amplitudes in the presence of uncertain parameter

In Figures 4.5(a) and 4.5(c) where $\omega=1$, roll responses of NLDI+MRC stay closest to the desired orientation trajectory while adaptation method also remain relatively close except at the peaks of the sinusoidal motion. The greatest error 
for these graphs are attained with Integral Backstepping technique. When the frequency of the sinusoidal motion is increased, at the amplitude value of 1 as in Figure 4.5(b), both NDI+MRC and IB lose their functionality while response of MRAC still tracks the desired trajectory. At a relatively lower amplitude value of 0.5 ; as can be investigated in Figure $4.5(\mathrm{~d})$, overall analysis is similar to that made for Figure 4.5(c) with an exception. The exception is the disrupted behaviour in the tracking of Integral Backstepping which bear more resemblance to the performance in Figure 4.5(b).

\subsection{Error Norms of the Simulation Results}

In order to find basis for the discussions about the simulation results in the previous chapters about sinusoidal and constant roll input types, 2-norms and absolute maximum values of state error vectors can be investigated. Table 4.5 includes the 2-norms of the error vectors while Table 4.6 includes the maximum absolute values of error vectors regarding a specified method for different scenarios and input cases. Specifically, in Table 4.5, it is clear that the maximum absolute values and 2-norms of roll errors in MRAC scheme are substantially smaller than those of IB and base controller under the existence of unknown disturbances. When the values regarding errors in roll states and uncertain parameters are explored, error norms of base controllers are intolerably higher than the others. 2-norms of roll error vectors increase 20 times for NLDI+MRC, approximately 37 times for IB and 15 times for MRAC in noisy and noiseless cases when the input type is changed from the step to the sinusoidal type. These drastic growths are not observed in the cases with disturbances and uncertainty. Another point that is worthy of mentioning is the stabilized levels of errors in

pitch and yaw for nearly all cases. The exceptions are the pitch response when the parameter uncertainty is present and the yaw response under the influence of unknown disturbances. The ineffectiveness of Integral Backstepping when giving a sinusoidal roll command to the system is apparent from the roll error column of 2-norms. Although the capabilities of base controller scheme and MRAC are around the same for the first two scenarios again for sinusoidal input, 
MRAC outperforms the other option when disturbances or uncertainties come into picture. Finally, the performance of Integral Back-stepping controller is higher in the pitch and yaw axes in which there are no commands given. However, this fact does not prevent the divergence of the method in the commanded roll axis. A final note is that the frequencies and amplitudes of all the sinusoidal commands given are respectively 2 and 1 . These values correspond to the error norms and maximum error values in Tables 4.5 and 4.6 .

Table 4.5: 2-Norm of the Error Vectors of the Control Methods for Different Scenarios and Inputs

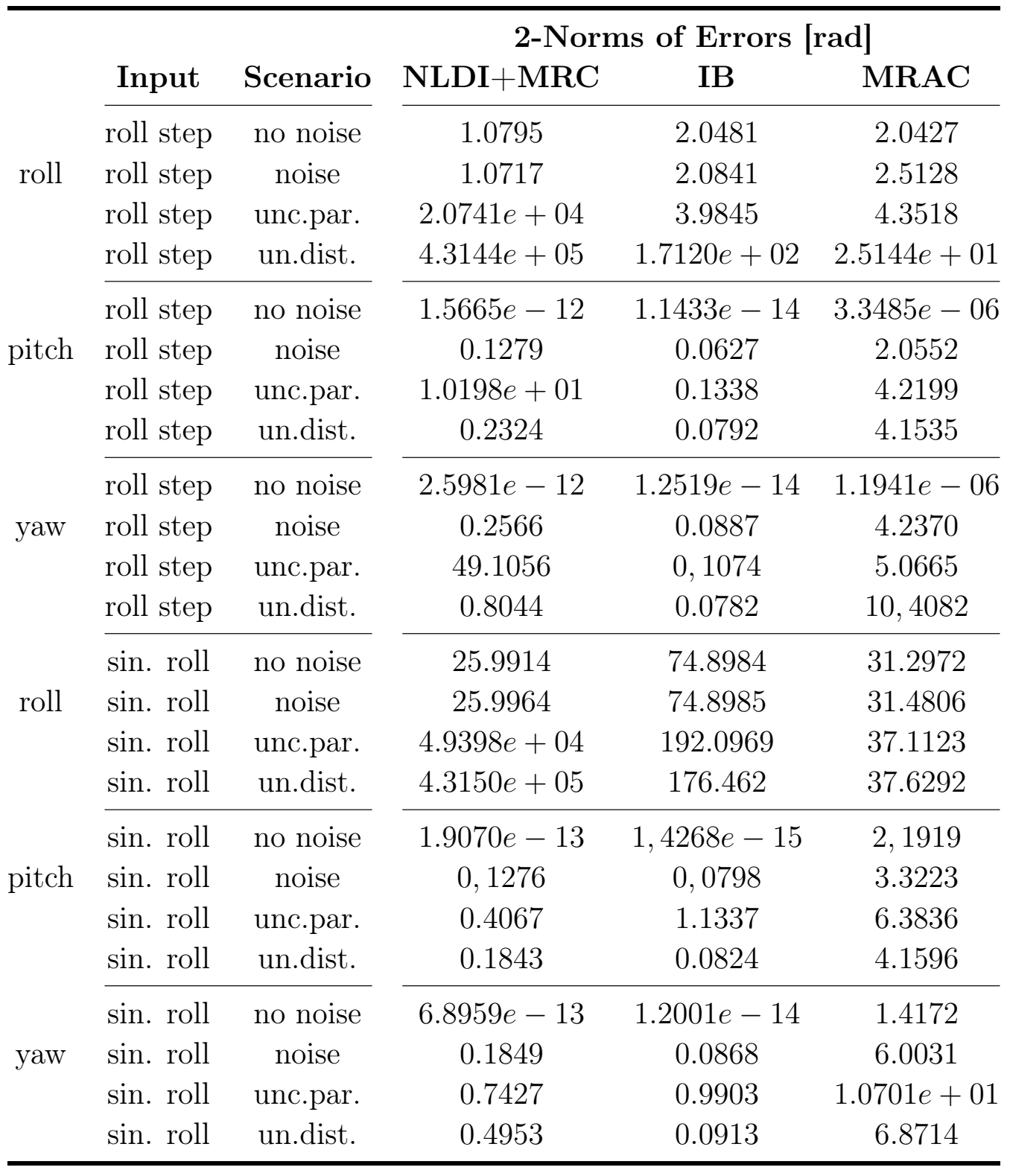


Table 4.6: Maximum Absolute Values of the Error Vectors of the Control Methods for Different Scenarios and Inputs

\begin{tabular}{|c|c|c|c|c|c|}
\hline & & & Max. Abs. & Values of Er & ors [rad] \\
\hline & Input & Scenario & $\mathrm{NLDI}+\mathrm{MRC}$ & IB & MRAC \\
\hline & roll step & no noise & 2.3947 & 4.5696 & 4.5577 \\
\hline roll & roll step & noise & 2.4070 & 4.5860 & 5.7923 \\
\hline & roll step & unc.par. & $2.7075 e+04$ & 8.7456 & 9.6859 \\
\hline & roll step & un.dist. & $4.3144 e+05$ & $1.7120 e+02$ & $2.5144 e+01$ \\
\hline & roll step & no noise & $1.5665 e-12$ & $1.1433 e-14$ & $1.2950 e-05$ \\
\hline pitch & roll step & noise & 0.1279 & 0.0802 & 4.4039 \\
\hline & roll step & unc.par. & 0.5121 & 0.2670 & 9.4877 \\
\hline & roll step & un.dist. & 0.2324 & 0.0792 & 4.1535 \\
\hline & roll step & no noise & $1.9032 e-11$ & $6.9956 e-15$ & $2.8225 e-06$ \\
\hline yaw & roll step & noise & 0.1840 & 0.0857 & 9.0514 \\
\hline & roll step & unc.par. & 2.4700 & 0.1605 & 12.5385 \\
\hline & roll step & un.dist. & 0.8044 & 0.0782 & 10,4082 \\
\hline & sin. roll & no noise & 0.2626 & 0.8026 & 0.4662 \\
\hline roll & sin. roll & noise & 0.2648 & 0.8017 & 0.4704 \\
\hline & sin. roll & unc.par. & $1.4595 e+03$ & 2.2652 & 0.7748 \\
\hline & sin. roll & un.dist. & $1.1819 e+04$ & 4.1910 & 0.6551 \\
\hline & sin. roll & no noise & $9.3067 e-15$ & $1.2096 e-16$ & 0.0725 \\
\hline pitch & sin. roll & noise & 0.0027 & 0.0013 & 0.0607 \\
\hline & sin. roll & unc.par. & 0.0145 & 0.0195 & 0.1859 \\
\hline & sin. roll & un.dist. & 0.0054 & 0.0018 & 0.1181 \\
\hline & sin. roll & no noise & $1,8809 e-14$ & $2.9698 e-16$ & 0.0496 \\
\hline yaw & sin. roll & noise & 0.0032 & 0.0015 & 0.1060 \\
\hline & sin. roll & unc.par. & 0.0158 & 0.0183 & 0.2682 \\
\hline & sin. roll & un.dist. & 0.0145 & 0.0023 & 0.1004 \\
\hline
\end{tabular}

\subsection{Discussion for Simultaneous Inputs in 2-Different Axes}

For a wider conception, the simulations held for some simultaneous input types can be beneficial to test the controllers. The results can be visited in Appendix A. Figures A.1, A.2, A.3 and A.4 demonstrate the responses when the system is given simultaneous step inputs in two different axes. Under the circumstance 
of step inputs in two axes irrespective of the existence of noise, the combination of non-linear dynamic inversion technique and model reference controller performs adequately well when compared to other options which oscillate before converging. However, under the existence of parameter uncertainty, this combination fails as in the single input case. For this specific case, both IB controller and MRAC function in an acceptable manner. Similar to the case with the single input, when an unknown disturbance is present in the system, MRAC still remains as the only functioning method. These deductions are attained from Figures A.1, A.2, A.3 and A.4 which are available in App. A. An overall summary attained via the findings in this section is again the vitality of the use of adaptation especially in the presence of uncertainty and disturbances.

\subsection{Simulation Results with Various Step-size Values}

In adaptation, the sensitivity of the simulation results to the selection of step-size is another topic that should be dissected. Table 4.7 shows the levels of step-sizes and the labels of graphs regarding these analyses which are conducted for MRAC in the existence of both parameter uncertainty and unknown disturbances where the opted inputs are constant roll and pitch commands.

Table 4.7: Various Step Sizes Used for Simulations

\begin{tabular}{llll}
\hline Step-sizes (sec): & $10^{-2}$ & $5 \cdot 10^{-3}$ & $10^{-3}$ \\
\hline
\end{tabular}

The general trend in Figures 4.6 is that the step size changes does not lead to significant impressions on the responses of the controllers. This deduction is distinctive in Figures 4.6(a), 4.6(c) and 4.6(e) regarding parameter uncertainty. Figure 4.6(b), 4.6(d) and 4.6(f) also supports the consistency of MRAC results with different step-sizes. The results with different step sizes just fit on the top of each other. Consequently, these inferences ensure the independence of the adaptation method from the time step. Besides, the frequencies around $100 \mathrm{~Hz}$ which are generally considered for real-world applications result in satisfactory outcomes for experimental aims. 


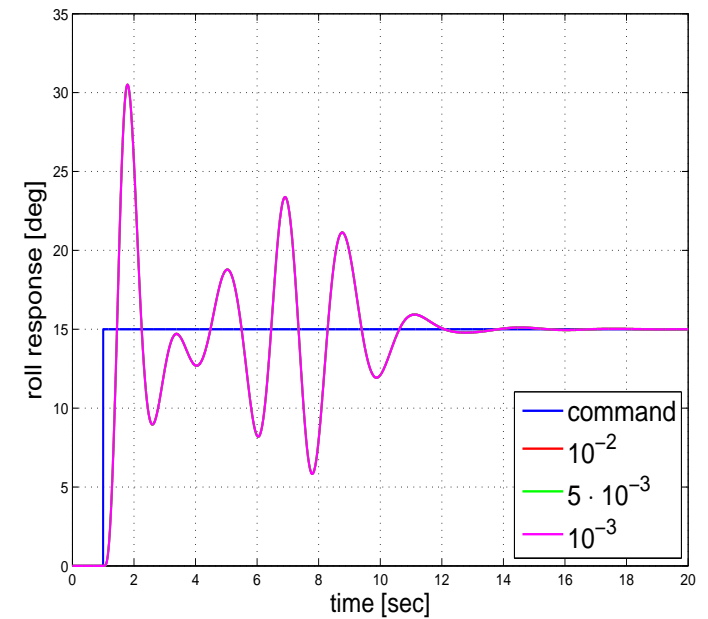

(a) Roll Response for Uncertain Parameter

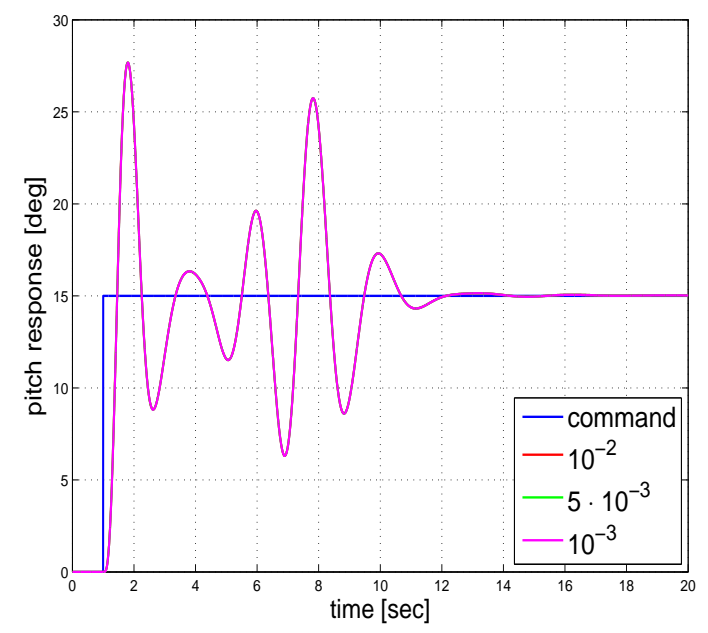

(c) Pitch Response for Uncertain Parameter

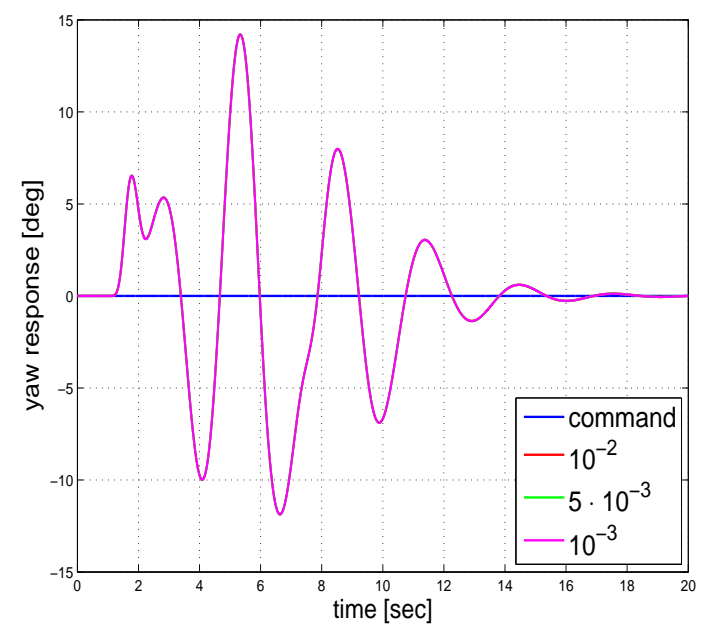

(e) Yaw Response for Uncertain Parameter

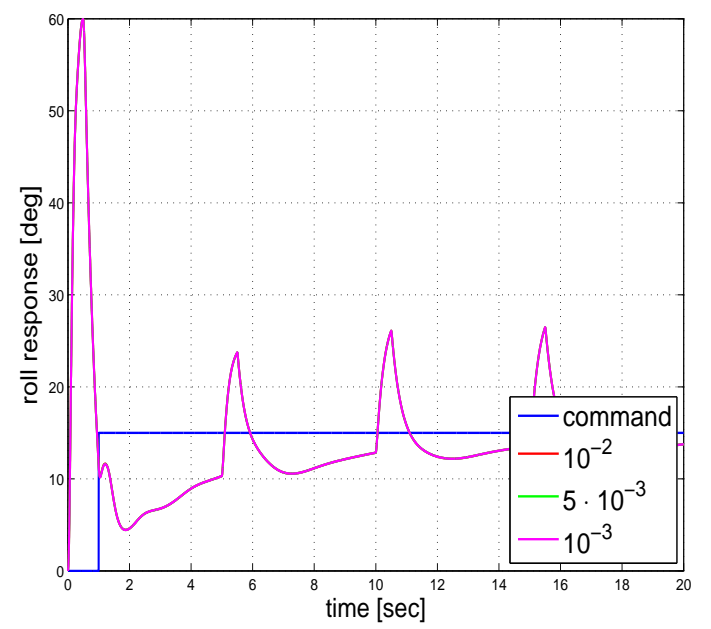

(b) Roll Response for Unknown Disturbance

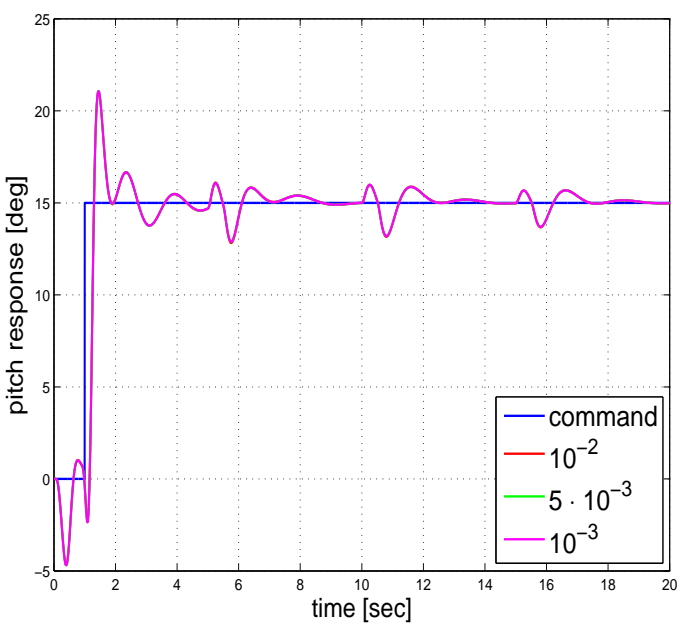

(d) Pitch Response for Unknown Disturbance

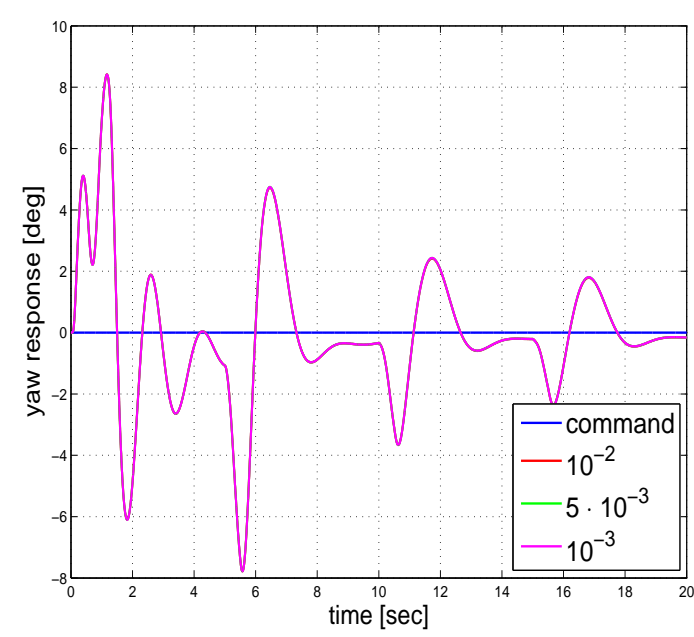

(f) Yaw Response for Unknown Disturbance

Figure 4.6: The Simulation of 2-Axis Fixed Orientation Commands in the Presence of Uncertain Parameters and Unknown Disturbances in Roll with Various Step-sizes 


\subsection{Simulations Results with Various Fixed-Step ODE Solver Meth- ods}

An analysis about the validity of the solver types used for the simulation should be carried out for the procedure. In a general simulation procedure, variable or fixed step-size solvers methods can be chosen dependent on the aims. The numeric methods considered for this study are fixed-step ODE solvers. In cases when the integration with a constant time step is present, some of the methods used may be more sensitive to the changes in step sizes or step size properties than others. In the simulations discussed until this point,a fixed-step ODE solver method of order 4 is used. Such a scheme is also named Runge-Kutta. Table 4.8 shows the solver types to be compared and Figures 4.7 illustrate the results attained with these ODE solvers for step roll command in the existence of parameter uncertainty. As shown in Figure 4.7(d) which is a zoom of 4.7(c). the difference between different types of solvers is around the level of $10^{-3} \mathrm{deg}$. By these observations, it is safe to assert that the simulation results of the adaptation are independent from the type of fixed-step numeric ODE solver used in the simulation procedure. Notice that the results with different solvers designated in the legend just fit on the top of each other. In another words, all lines in the graphs nearly correspond to each other from the large scale of Figures 4.7(a), 4.7(b) and 4.7(c), That is why only the result of Runge-Kutta method seems visible in Figures 4.7(a), 4.7(b) and 4.7(c), No other lines with any other colors regarding other methods is therefore invisible. A final note in this section is the step size used which is $10^{-3}$ sec.

Table 4.8: Comparison of Fixed-Step ODE Solver Types for Simulation

\begin{tabular}{ccc}
\hline Order of Method & & Solver Types \\
\cline { 1 - 1 } 4 & Runge - Kutta \\
2 & Heun \\
3 & Bogacki - Shampine \\
5 & Dormand - Prince \\
\hline
\end{tabular}




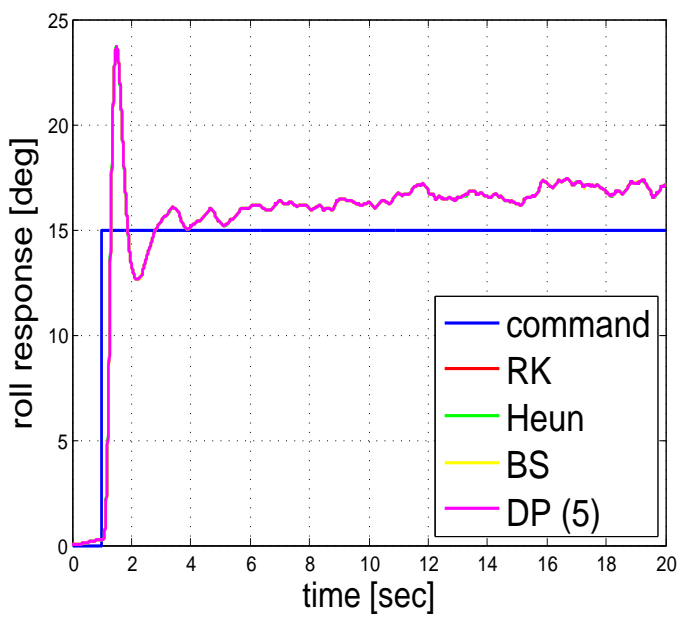

(a) Roll Response

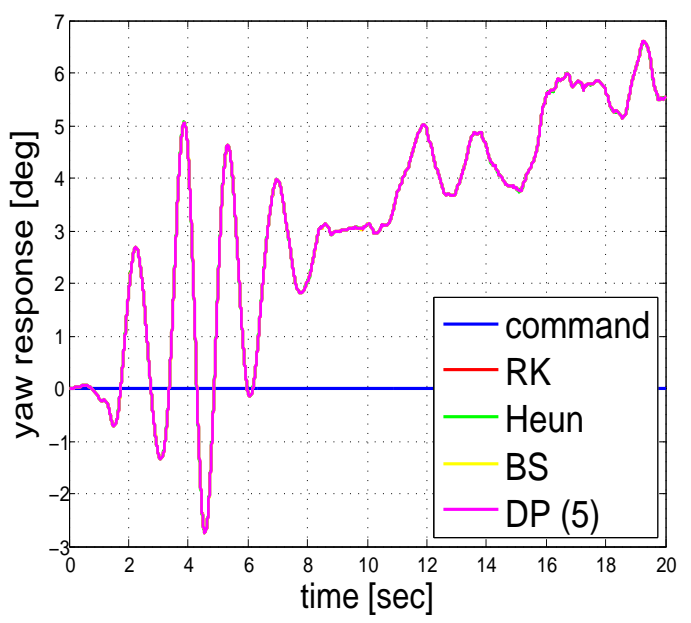

(c) Yaw Response

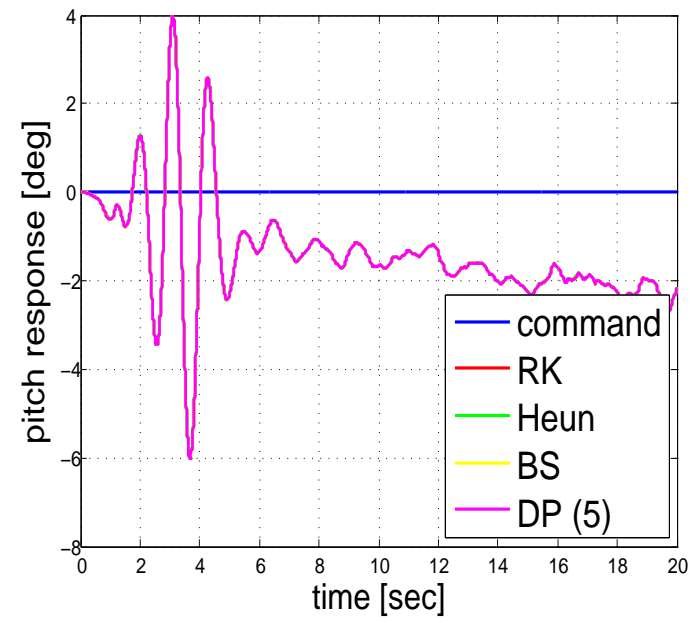

(b) Pitch Response

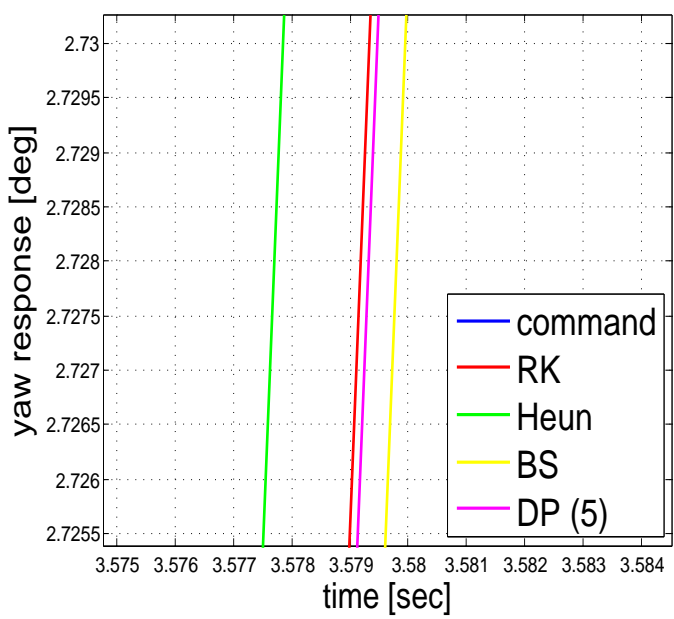

(d) Zoom

Figure 4.7: Simulation results of the constant orientation tracking in the existence of parameter uncertainty via various Runge-Kutta methods

\subsection{Simulations Results with Various Levels of Disturbances}

Instead of only one set, using several sets of unknown disturbances may give an idea about the boundaries of the capabilities of the adaptive controller and the range where the adaptation operates well. The input type chosen for this section is a step roll command. At this point, let remember the definition for unknown disturbances. In the simulation, the disturbance is defined as a pulse wave represented by an amplitude, $A_{\text {pulse }}$, a period, $T_{\text {pulse }}$, width, $w_{\text {pulse }}$ and a 
phase, $P_{\text {pulse }}$. Note that $T_{\text {pulse }}$ is $5 \mathrm{sec}, w_{\text {pulse }}$ is $0.5 \mathrm{sec}$ and $P_{\text {pulse }}$ is $0 \mathrm{sec}$. Table 4.9 contains amplitude levels for the disturbances considered within this section.

Table 4.9: Disturbance Levels for the Case with Various Unknown Disturbances

\begin{tabular}{|c|c|}
\hline Set Number & Disturbance Level $\left(A_{\text {pulse }}\right)$ \\
\hline dist.1 & 0.1 \\
\hline dist.2 & 0.25 \\
\hline dist.3 & 0.4 \\
\hline dist.4 & 0.5 \\
\hline
\end{tabular}

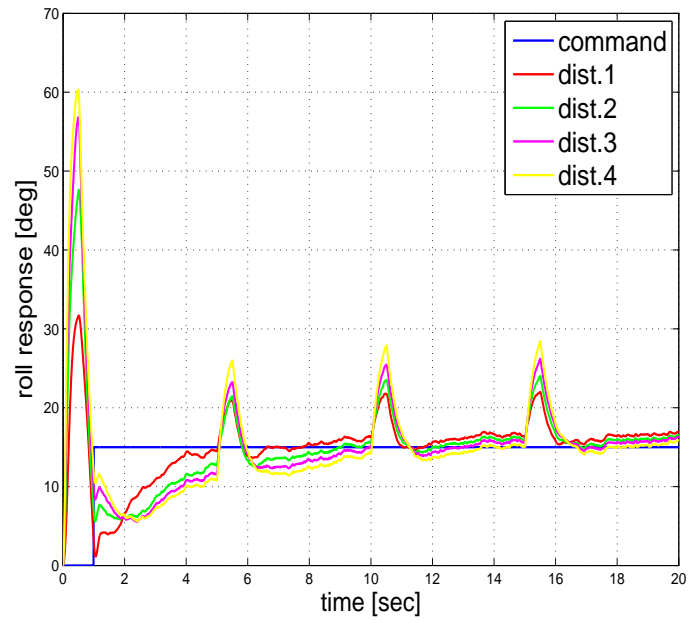

(a) Roll Response

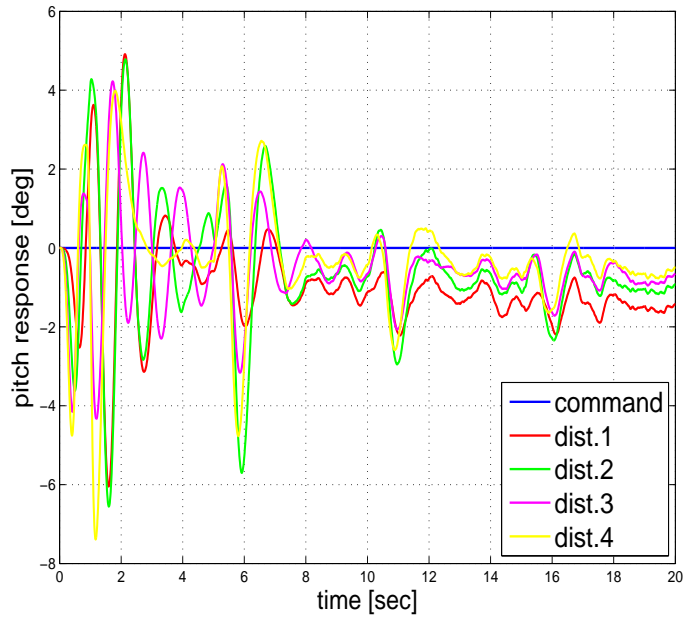

(b) Pitch Response

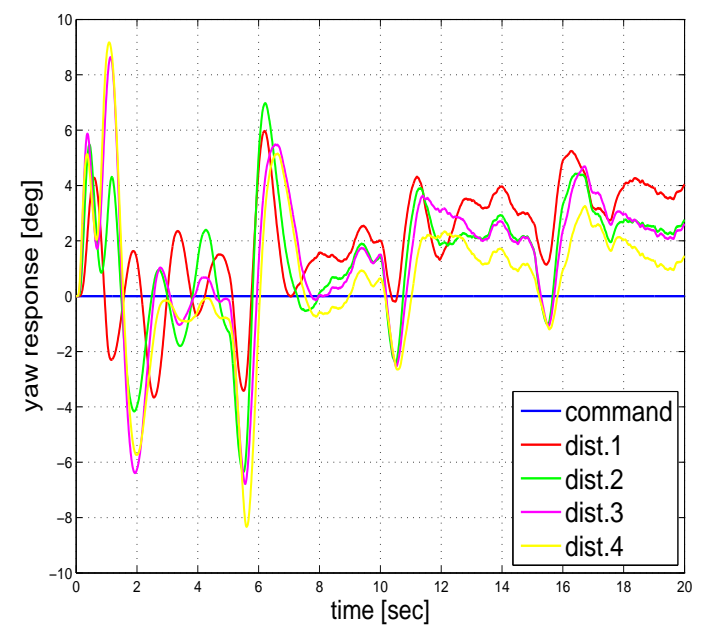

(c) Yaw Response

Figure 4.8: Simulation results of fixed angular orientation in the presence of various levels of unknown disturbances 
Investigating the peaks caused by the pulse waves in Figure 4.8(a), it is clear that the greater amplitude levels cause these peaks to reach a greater level. Especially, the first peak caused by the initial wave is 5 times higher than the successive waves. Moreover, the peak in the roll response caused by the unknown disturbance in rolling moment is 10 degrees for the label dist- 4 and 6 degrees for the label dist-1 which are successively maximum and minimum amplitudes among the roll response of all cases. In Figures 4.8(b) and 4.8(c), the responses in pitch and yaw responses caused by disturbances do not point out much change in behaviour due to changing amplitudes.

\subsection{Simulations Results with Various Levels of Uncertain Parame- ters}

Based on the same notion benefited in the previous section, defining several sets for parameter uncertainty may help while deciding the effectiveness of the adaptation as well. The parameters of concern in this section are moments of inertia of the platform. The true values of the moments of inertia that are assumed for the quadrotor are labelled as $I_{x, y, z}$. The coefficient of these inertias in the parameter uncertainty level column of Table 4.10 indicates the set of values assigned for a new simulation. The results can be explored in Figures 4.9. The input command considered for this section is a step roll command again. As can be detected in Figures 4.9(a), 4.9(b) and 4.9(c), the state responses with set number 1 containing the values of a quadrotor platform 5 times higher in all axes result in oscillatory behaviour although roll state finally remains bounded. It is clear in all the figures that the error between the desired trajectory and the state responses drops off as the moments of inertia are reduced. The force command given to the right motor of the quadrotor is shown in Figure $4.9(\mathrm{~d})$. Apparent from the chosen rotor in Fig. 4.9(d), there is no saturation observed in the command although the oscillatory behaviour of the adaptive controller is clear when dealing with the high level of uncertainties. 
Table 4.10: Uncertainty Levels for the Case with Various Uncertain Parameters

\begin{tabular}{|c|c|}
\hline Set Number & Parameter Uncertainty Level \\
\hline set.no $=1$ & $5 \cdot I_{x, y, z}$ \\
\hline set.no $=2$ & $I_{x, y, z}$ \\
\hline set.no $=3$ & $0.2 \cdot I_{x, y, z}$ \\
\hline set.no $=4$ & $0.1 \cdot I_{x, y, z}$ \\
\hline
\end{tabular}

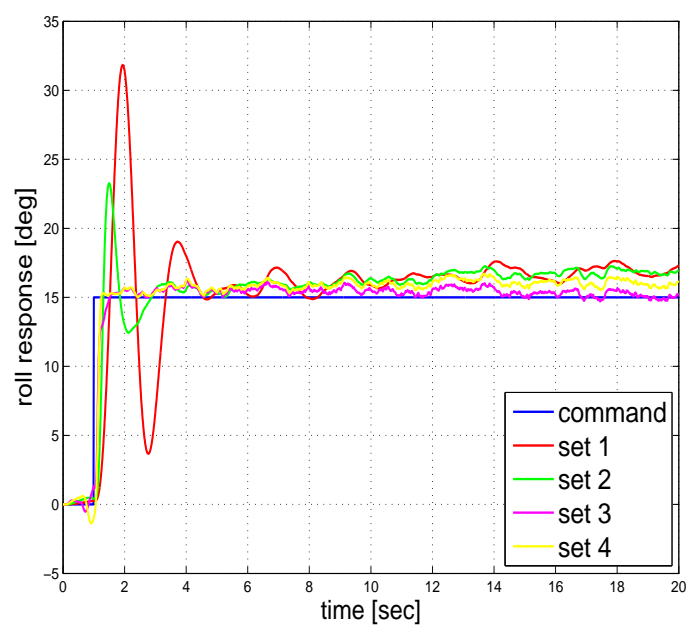

(a) Roll Response

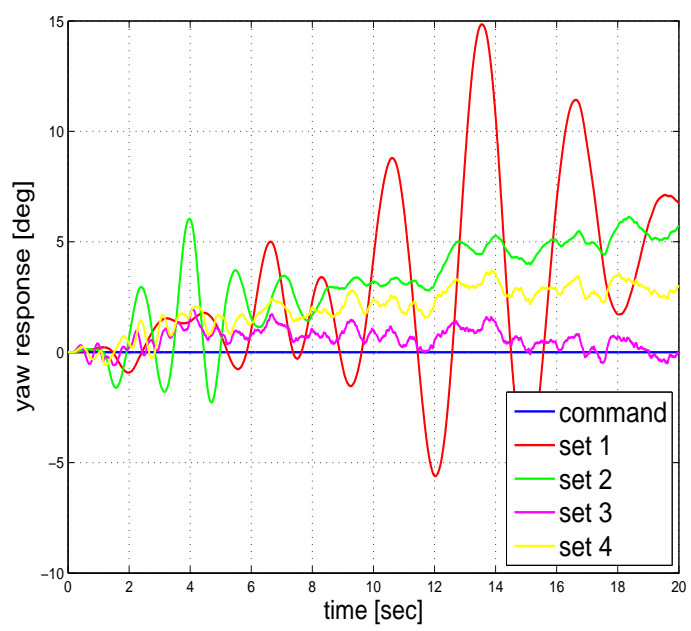

(c) Yaw Response

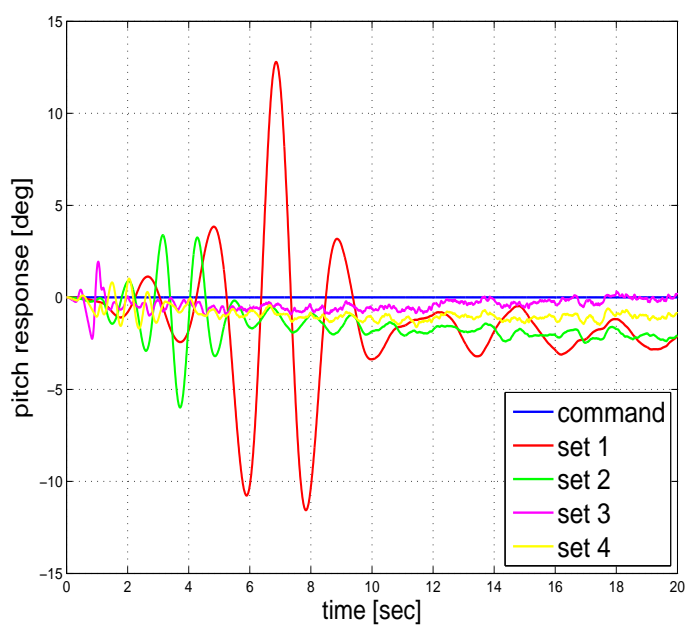

(b) Pitch Response

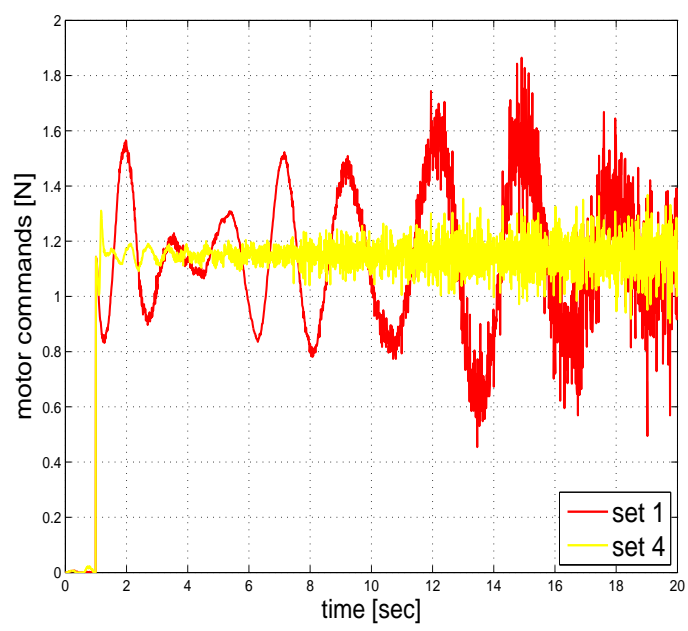

(d) Motor Command

Figure 4.9: Simulation results of fixed angular orientation in the presence of various uncertain parameters 


\section{CHAPTER 5}

\section{RESULTS AND DISCUSSION FOR ADAPTATION AND ROBUSTNESS MODIFICATIONS}

This chapter includes the discussions about the convergence of adaptation gains and the influence of the use of robustness modifications to MRAC.

\subsection{Adaptation Gains for Simulations}

The adaptation gains to be investigated within this section belong to the results attained in the previous chapter. Figure 5.1 visualizes the variation of adaptation gains with time in the specified states and cases. Notice that the legends labelled as fore, aft, left and right indicate the motors of the quadrocopter. In Figures $5.1(\mathrm{a})$, $5.1(\mathrm{e})$ and $5.2(\mathrm{a})$, the fundamental element causing the gains to remain bounded is clearly the non-existence of noise in the simulations. Regardless of the presence of unknown disturbance or parametric uncertainty, the extension with the noise elements leads to the drift-away of the gain elements in a specific time duration. The outcomes shown in Figures 5.1(c), 5.1(d), 5.2(c) and 5.2(d) are also consistent with the results of the experiments held in [133]. Via the modification of MRAC with Dead-Zone, the controller is transformed into a structure where the adaptation is switched off below an error criteria and the adaptation gains remain unforgotten. When the drift-away due the Dead-Zone modification is the scope of interest, as shown in Figures 5.3(e) and 5.3(f), the problem of drifting-away continues with noise addition. Without noise, however, the gains remain bounded as in Figures 5.3(a), 5.3(b), 5.3(c) and 5.3(d). 


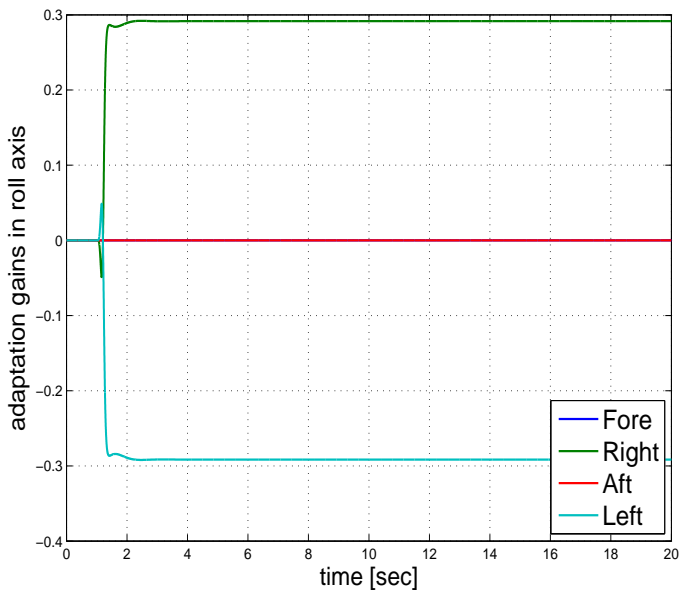

(a) Roll Axis Gains with no noise

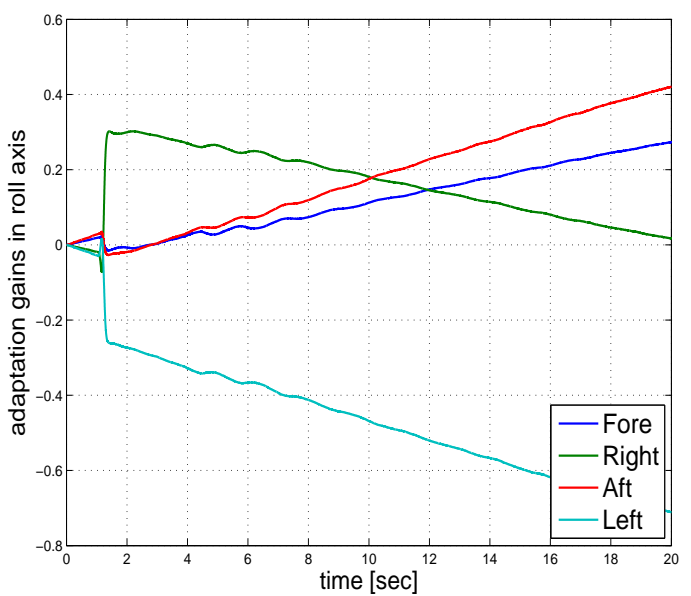

(c) Roll Axis Gains with noise addition

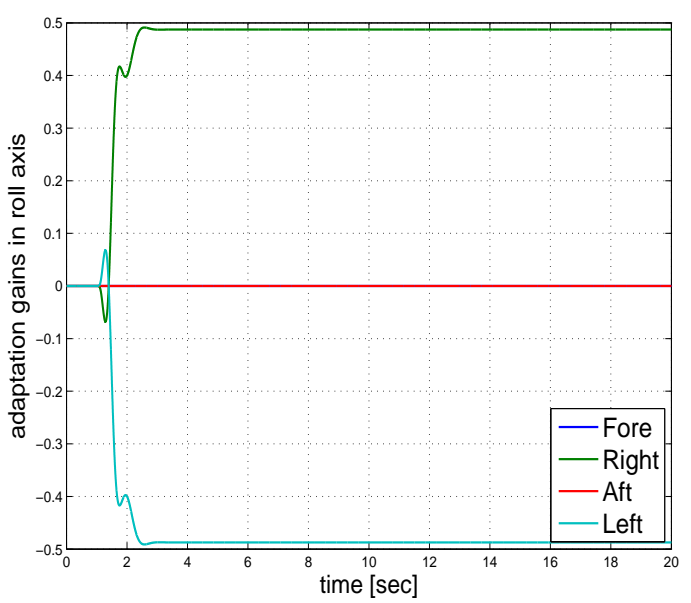

(e) Roll Axis Gains in the presence of parameter (f) Pitch Axis Gains in the presence of parameter uncertainty

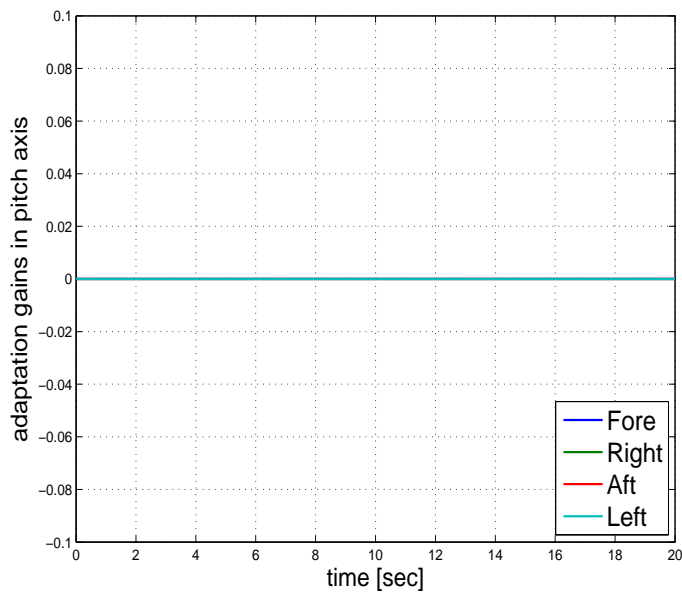

(b) Pitch Axis Gains with no noise

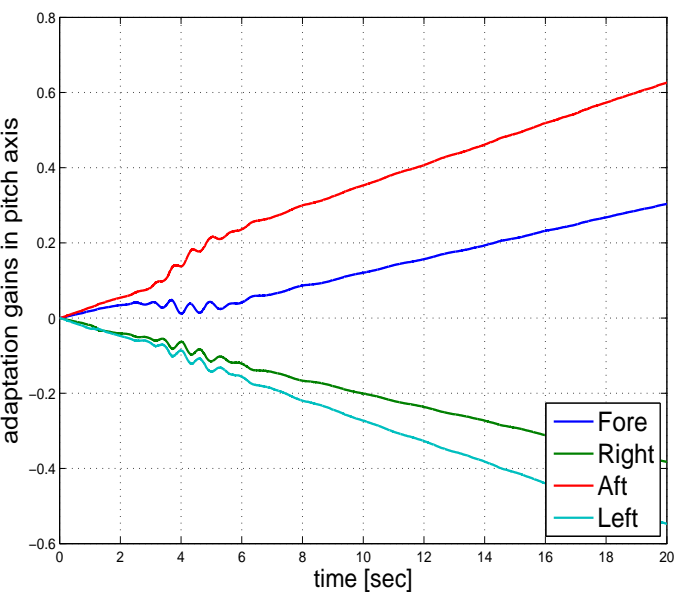

(d) Pitch-Axis Gains with noise addition

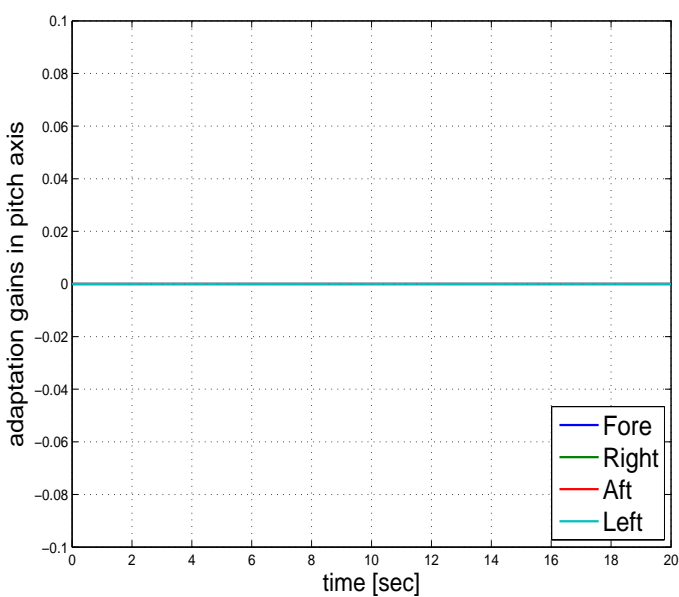
uncertainty

Figure 5.1: MRAC Adaptation Gains related to State Outputs in the simulation of attitude control for roll step-input tracking 

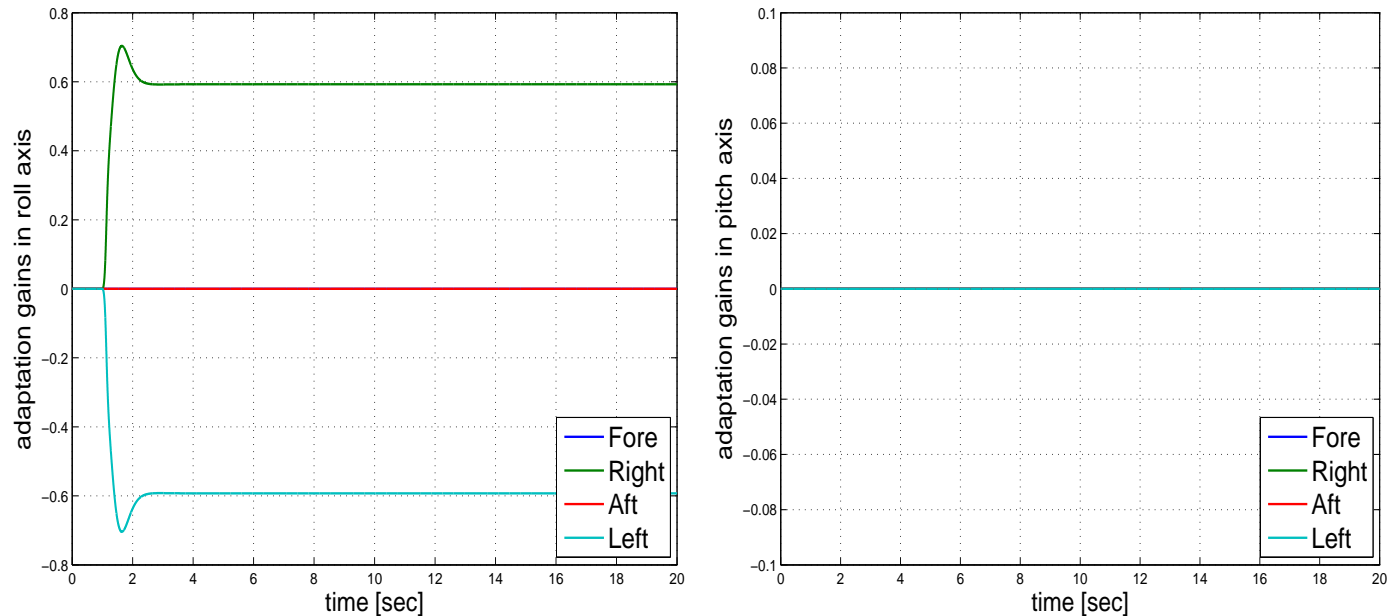

(a) Roll Axis Gains in the presence of unknown

(b) Pitch Axis Gains in the presence of unknown disturbance disturbance
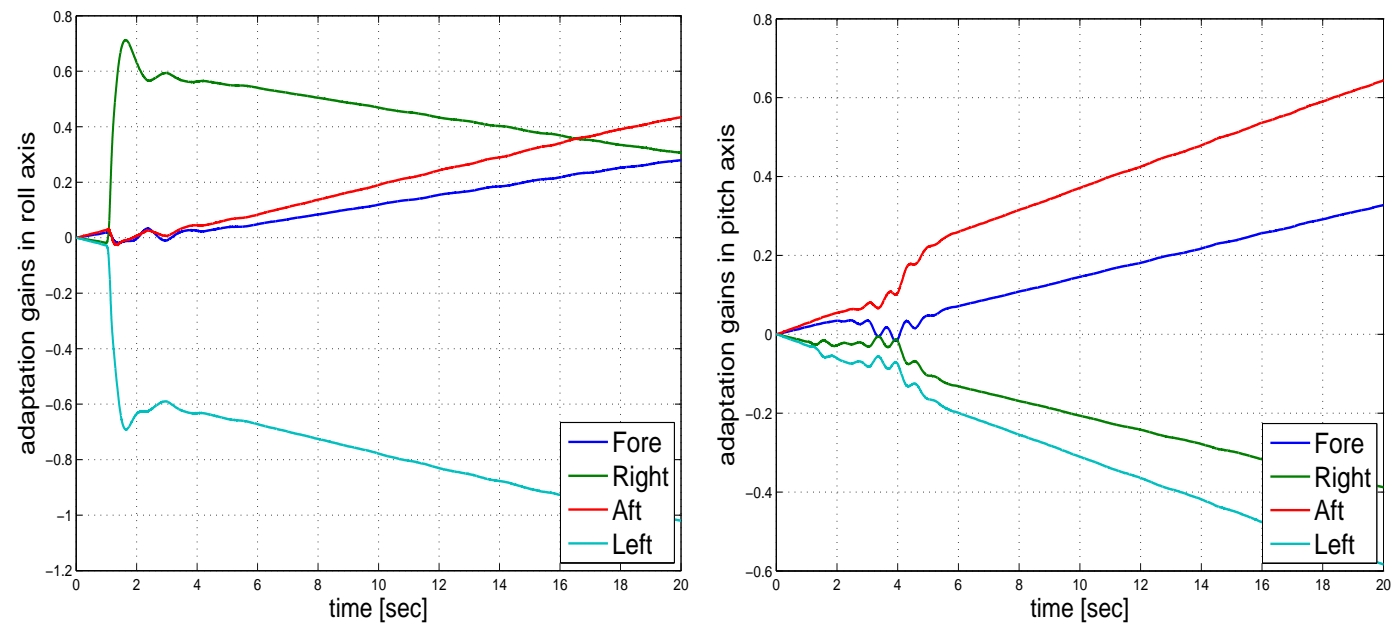

(c) Roll Axis Gains in the presence of unknown disturbance and noise

(d) Pitch Axis Gains in the presence of unknown disturbance and noise

Figure 5.2: MRAC Adaptation Gains related to State Outputs in the simulation of attitude control for roll step-input tracking (continued) 


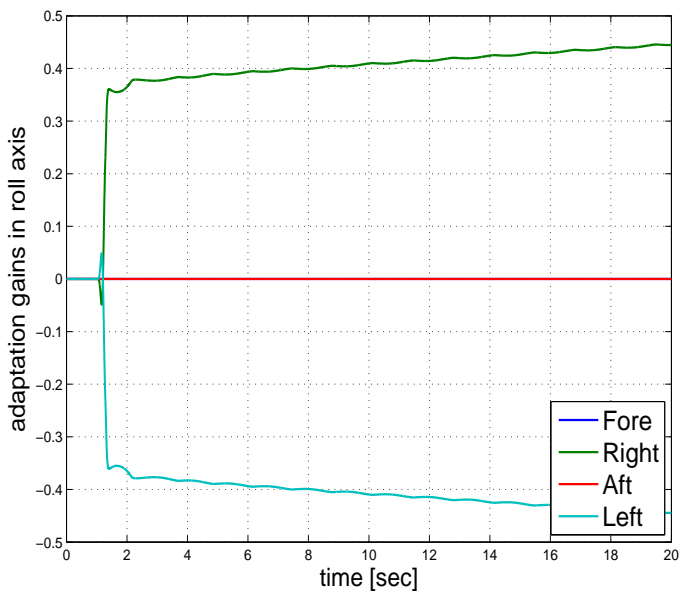

(a) Roll Axis Gains with no noise

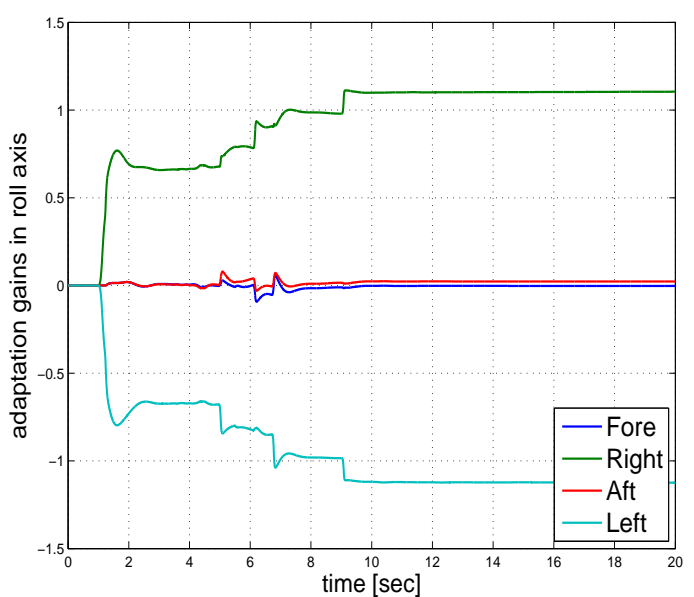

(c) Roll Axis Gains in the presence of unknown disturbance

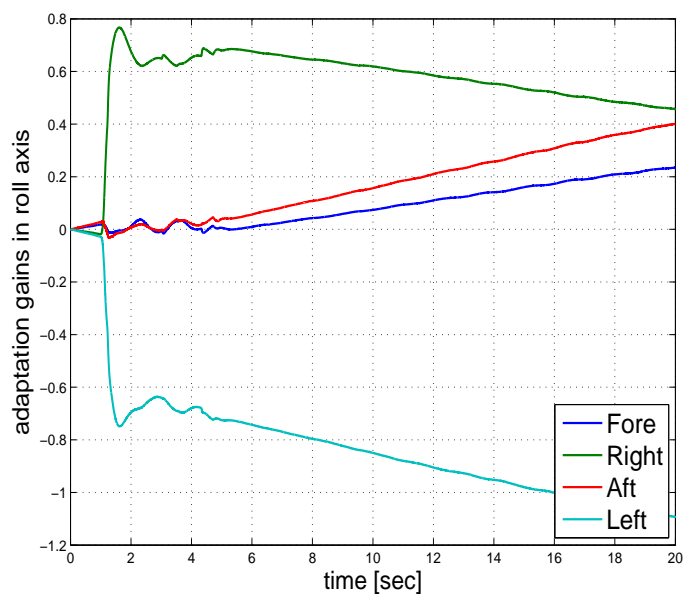

(e) Roll Axis Gains in the presence of unknown disturbance and noise

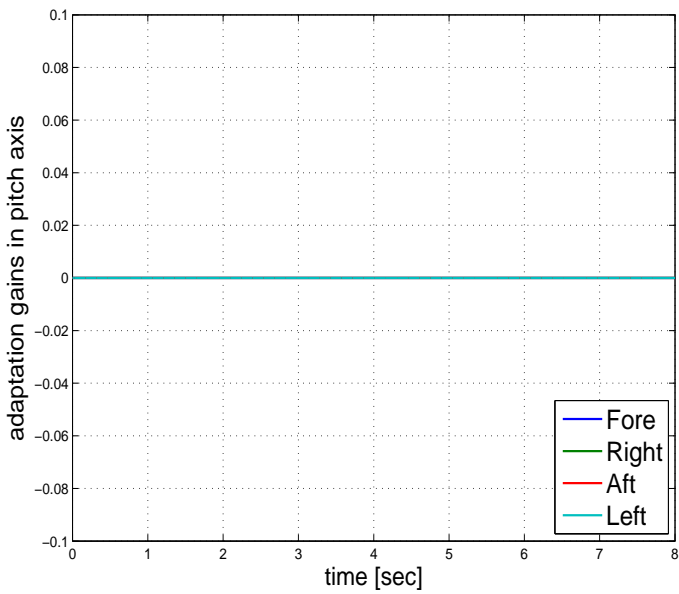

(b) Pitch Axis Gains with no noise

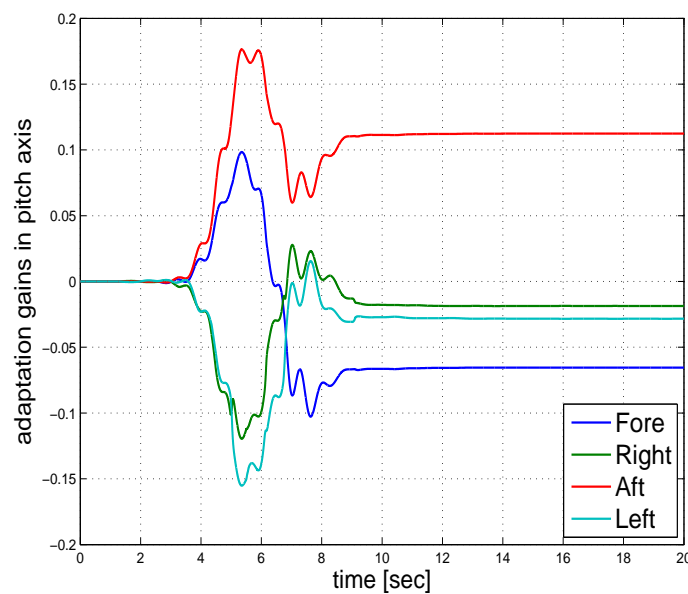

(d) Pitch-Axis Gains in the presence of unknown disturbance

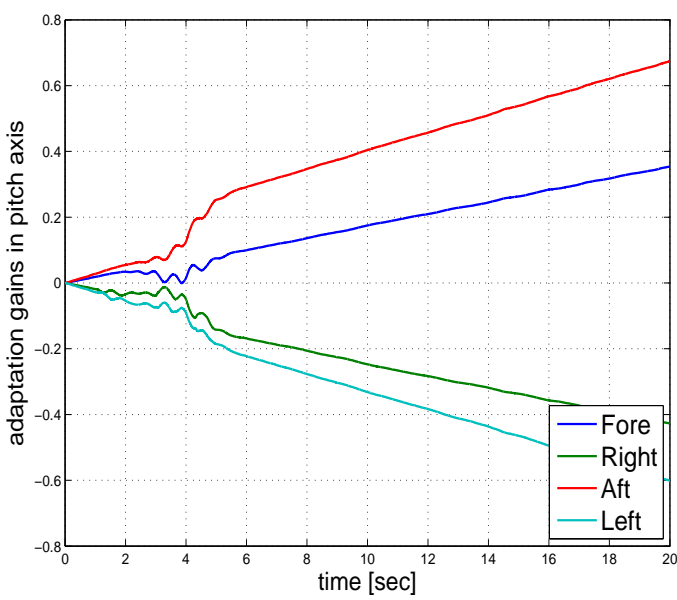

(f) Pitch Axis Gains in the presence of unknown disturbance and noise

Figure 5.3: MRAC+Dead-Zone Modification Adaptation Gains related to State Outputs in the simulation of attitude control for roll step-input tracking 


\subsection{Simulation Results and Discussion for Robustness Modifications and Unmodified Adaptation}

For the discussion of the influence of the robustness modifications to the model reference adaptive control, the input cases in Table 4.3 are considered. Note that commands in both 1- and 2-axes are considered for step type input. The commands in all other axes that are not designated in Table 4.4 are assumed to be zero.

Table 5.1: Input Types

\begin{tabular}{|c|c|c|}
\hline Input Type & Command and Axes & Input Value [deg] \\
\hline Constant Trajectory & Step Roll & 25 \\
\hline Constant Trajectory & Step Roll and Pitch & 25,25 \\
\hline
\end{tabular}

Table 4.7 includes information about the scenarios and the input types for those cases. The corresponding figures are also shown in Table 4.7.

Table 5.2: Simulation Scenarios with their figure numbers

\begin{tabular}{|c|c|c|}
\hline Input \& Scenario & Step Roll & Step Roll and Pitch \\
\hline noise + unknown dist. & Fig. 5.4 & Fig. C.1 \\
\hline noise + unknown dist. + unc.par. & Fig. $\overline{\mathrm{C} .2}$ & Fig. 5.5 \\
\hline
\end{tabular}

The modification types that are considered in a specific figure are summarized in Table 4.8. The robustness modifications that are utilized in this section are Dead-Zone extended MRAC, e-modification, $\sigma$-modification and Dead-Zone extended e-modification. In order to achieve trustworthy results from the comparison of modification methods, the unmodified MRAC is also considered.

Table 5.3: Modification Types in Figures

\begin{tabular}{|c|c|}
\hline $\mathrm{Col}$ & ers \\
\hline $\mathrm{M}$ & \begin{tabular}{|l|l|l|l|l|l|l|l|l|}
.4(a) & $5.4(\mathrm{~b})$ & C.1(a) & C.1(b) & C.2(a) & C.2(b) & $5.5(\mathrm{a})$ & $5.5(\mathrm{~b})$ \\
\end{tabular} \\
\hline MRA & 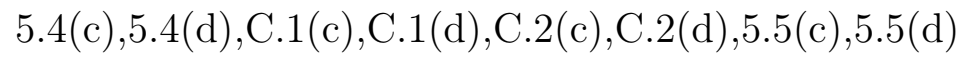 \\
\hline$\sigma-, \mathrm{e}-, \mathrm{e}-\mathrm{mod}+\mathrm{DZ}$ & \begin{tabular}{|l|l|l|l|l|l|l|l|l|l|l|l|l|l|l}
$5.4(\mathrm{e})$ & $5.4(\mathrm{f})$ & $\mathrm{D} .1(\mathrm{a})$ & $\mathrm{D} .1(\mathrm{~b})$ & $\mathrm{C} .2(\mathrm{e})$ & $\mathrm{C} .2(\mathrm{f})$ & $5.5(\mathrm{e})$ & $5.5(\mathrm{f})$ \\
\end{tabular} \\
\hline
\end{tabular}


Figures 5.4, C.1, C.2 and 5.5 include the errors in the indicated states. The legends for controller types are also indicated in the graphs as well as in Table 4.8 .

As indicated in the related sub-figures of Figures 5.4, C.1, C.2, and 5.5, $\sigma$ modification do not function as desired and should be eliminated from the list of robustness modifications to be used. As mentioned before, the main advantage of using a dead-zone switching is to prevent from the forgetting of adaptation gains. The results in Figures 5.4(c), 5.4(d), C.1(c), C.1(d), C.2(c), C.2(d), 5.5(c), and $5.5(\mathrm{~d})$ point out that the performance of a dead-zone modification remains nearly the same as the unmodified controller for all the cases.

Finally, taking the e-modification method into the scope of the discussion, the main deduction is that, only in the existence of more than one scenario element, the significance of e-modification could be put forward more explicitly. Focusing on Figures 5.4(b) and C.1(b), the e-modification holds better performance, especially when the errors in the pitch axis is considered. The results in roll axis can be claimed to remain similar for unmodified and e-modified MRACs. A drawback discovered in the results for e-modification is the oscillatory behaviour. Nevertheless, the remaining of the behaviour within the levels of several degrees and the fact that better choice of gains may hold better behaviour compensate the influences of this discovery on the system. Similar to the case in DeadZone modification to the pure MRAC, the Dead-Zone modification extension to the e-modified MRAC does not show visible improvements in the performance; however, conceptually, it prevents the gains from being drawn to zero here as well. 


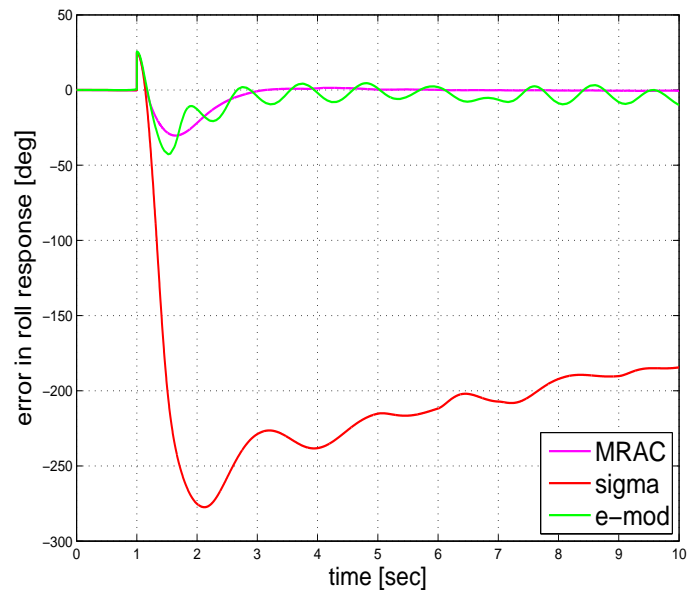

(a) Error in Roll Response

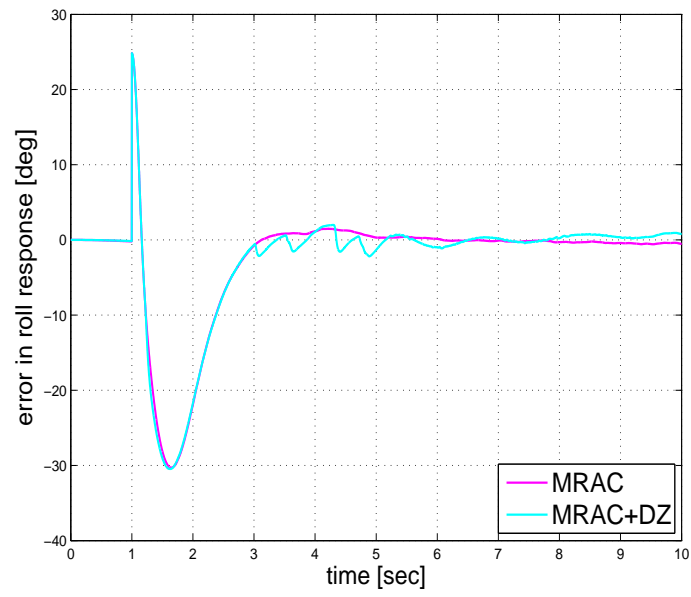

(c) Error in Roll Response

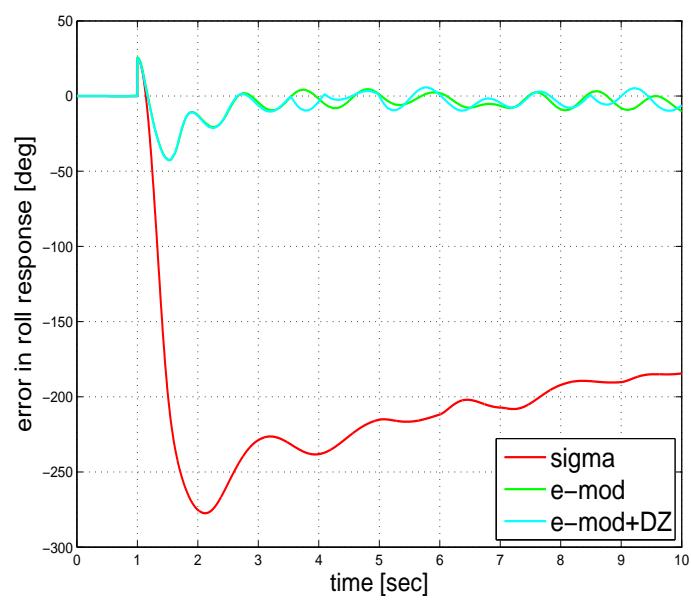

(e) Error in Roll Response

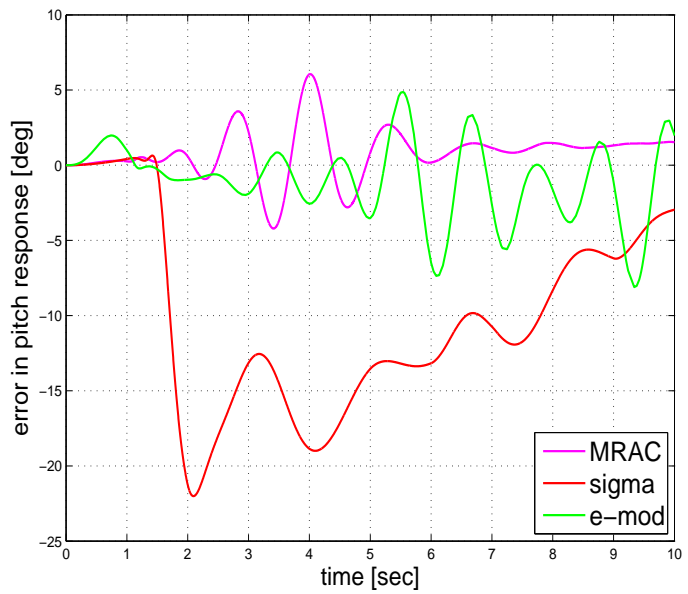

(b) Error in Pitch Response

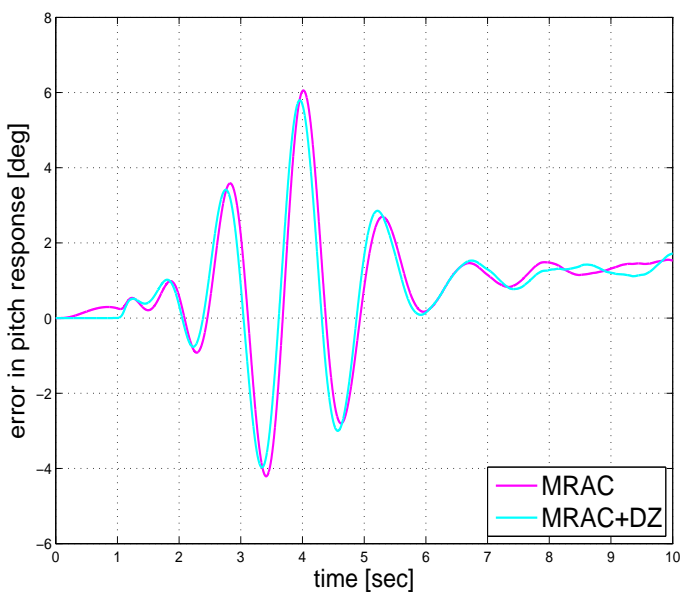

(d) Error in Pitch Response

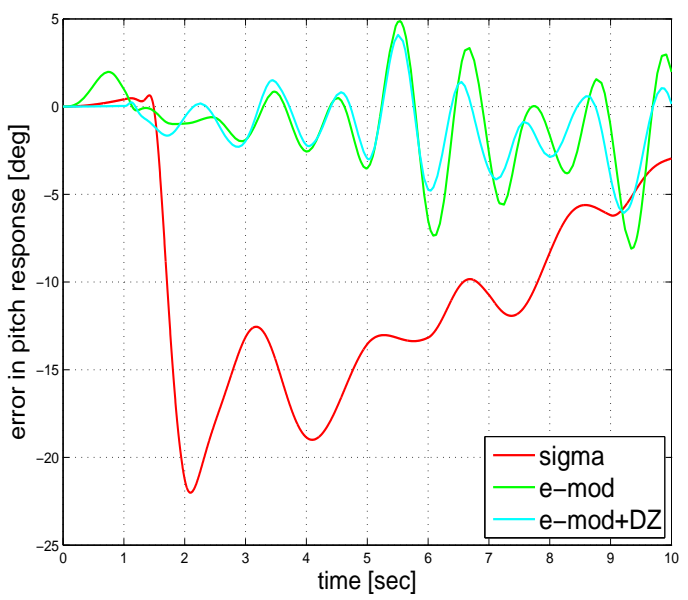

(f) Error in Pitch Response

Figure 5.4: State Error Responses of Various MRAC Robustness Modifications for Step Roll-Input Trajectory Tracking in the presence of noise and unknown disturbance 


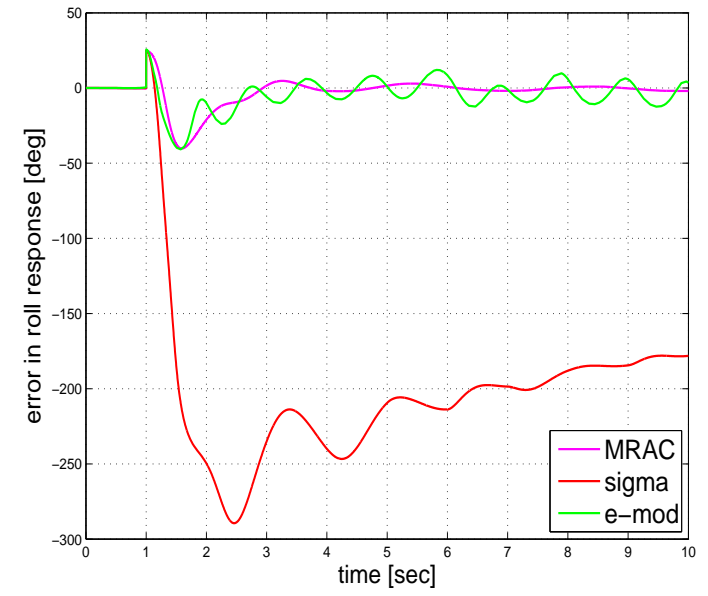

(a) Error in Roll Response

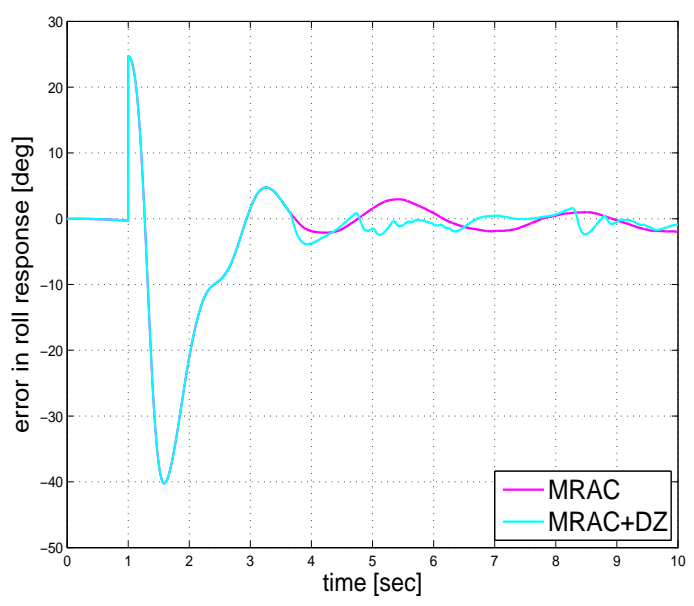

(c) Error in Roll Response

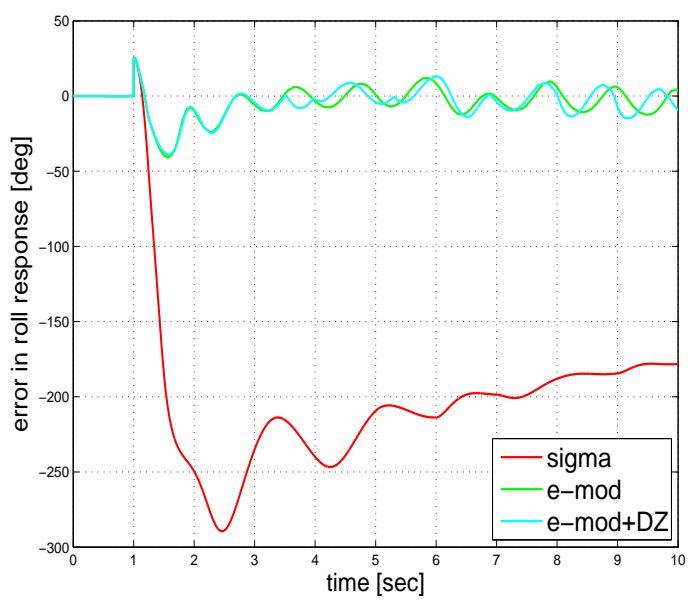

(e) Error in Roll Response

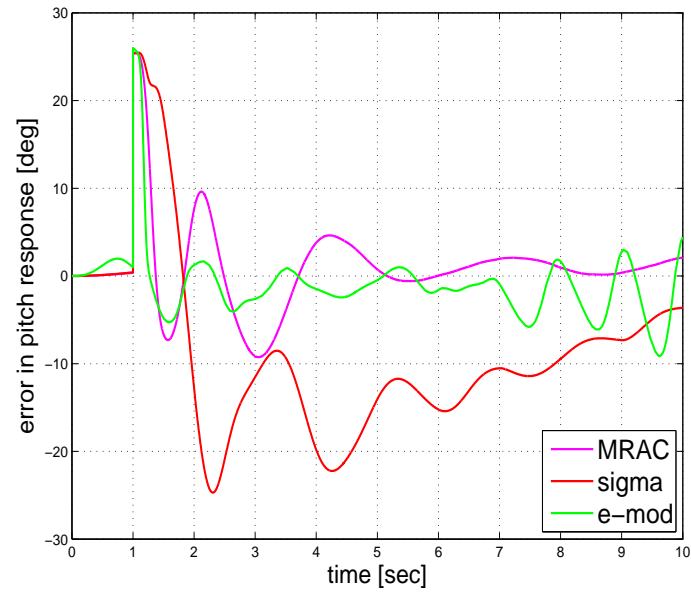

(b) Error in Pitch Response

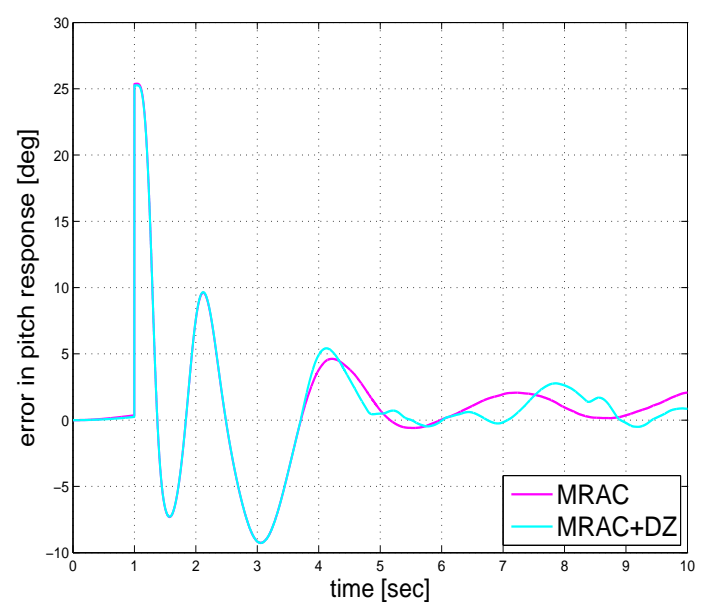

(d) Error in Pitch Response

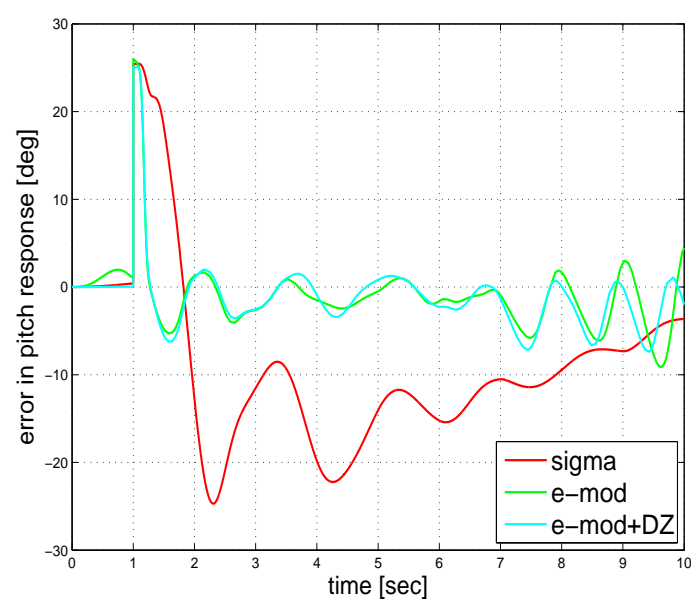

(f) Error in Pitch Response

Figure 5.5: State Error Responses of Various MRAC Robustness Modifications for Step Roll and Pitch-Input Trajectory Tracking in the presence of noise, parameter uncertainty and unknown disturbance 


\section{CHAPTER 6}

\section{CONCLUSION}

This thesis presents attitude controller options for the quadrotor dynamical system in the presence of 1) excellent sensors, 2) noisy measurements, 3) the presence of parameter uncertainty and 4) unknown disturbances. Firstly, Non-linear Dynamic Inversion is employed as a feedback linearization tool to transform the system into a non-linear form where linear tools are also available for use in attitude control structure. Nevertheless, the requirement of precise system knowledge forced the research to broaden to the field of adaptive control that suggests a systematic plan for automatic adjustment of controllers in real time, in order to sustain a desired level of control system performance when the system parameters of the dynamic model are not precisely known over time. Using Model Reference Adaptive Control, the controller is designed to form a cascaded structure. Composed of two loops, purely trigonometric attitude loop and rate loop based on uncertainties and an adaptive law, this controller is deduced to be adequately agile in parameter change compensations in real flight. Furthermore, pseudo-control hedging is inserted into these two controllers with an aim to solve the wind-up problem of integrators in error controllers as well as the the unlimited growth of adaptive gains triggered by actuator saturations. The main motivation of this is indeed to slow down the reference model exactly as much as the reaction deficit of the command input so as to hide the adaptive elements from the system characteristics and the saturations. This way, the error controller is not allowed to give the rate command signals the rate loop cannot follow. As a compelling member of model-based control family, Integral Back-stepping Technique is explored and studied for performance discussion. 
The simulation results show that all controllers operate exceptionally in noiseless and noisy scenarios. Under the cases where high-level parameter uncertainties or unknown disturbances exist, however, the performance loss of base controller and Integral Back-stepping are obvious. In coherence with its purpose of integration into the controller, only adaptive algorithm survives such cases and outperforms the others. Nonetheless, for other controller options, the explicit information about important system parameters and correct controller gains need to be known for functioning as desired. All these outperforming deductions are supported by 2-norms and maximum absolute value of error vectors in the commanded state axis numerically.

This confirms the strength of adaptation laws for the cases including the existence of disturbances, non-modelled dynamics and unmatched uncertainties. Furthermore, the boundedness of the adaptive gain parameters are provided as well. The modifications of dead-zone implementation, $\sigma$ and e-modifications are investigated so as to improve the robustness of the adaptation. Particularly, the use of e-modification and dead-zone implementation together holds potential for experimentation.

The future work of this thesis is the experimental verification of the methodologies discussed. The validation of current simulations may be finalized with the addition of flight test data. The experimental judgements on parameter selections can be addressed as well. The experiments may also include information on parameter designation, performance under external real disturbance and input signal tracking. The main motivation for future work is that the experimentation of attitude controllers sets up the basis for future research aims such as target tracking, obstacle avoidance and formation flight. 


\section{REFERENCES}

[1] Homer, R. Fagles, and B. Knox, The Iliad. Penguin Books, Penguin Books, 1991.

[2] I. Asimov, I, Robot. The Robot Series, Random House Publishing Group, 2004.

[3] K. Apek, R.U.R (Rossum's Universal Robots). Echo Library, 2010.

[4] B. Clough, "Metrics, Schmetrics! How the Heck do you Determine a UAV's Autonomy Anyway?," in Proceedings of the 2002 Performance Metrics for Intelligent Systems Workshop, (New York, NY, USA), NIST Special Publication, 2002.

[5] R. Pfeifer and C. Scheier, Understanding Intelligence. Bradford Books, MIT Press, 2001.

[6] E. Papadopoulos, "Heron of Alexandria (c.10-85 AD)," Distinguished Figures in Mechanism and Machine Science: Their Contributions and Legacies, History of Mechanism and Machine Science, vol. 1, pp. 217-245, 2007.

[7] T. B. Musa and D. R. Hill, The Book of Ingenious Devices: Kitab AlHiyal. By The Banu (sons Of) Musa Bin Shakir. D. Reidel Publishing Company, 1979.

[8] J. Adamy and A. Flemming, "Soft Variable Structure Controls: A Survey," Automatica, vol. 40, pp. 1821-1844, nov 2004.

[9] D. R. Hill, "Mechanical Engineering in the Medieval Near East," Scientific American, vol. 264, pp. 100-105, may 1991.

[10] M. Taddei, Da Vinci's Robots. New mechanics and new automata found in codices. Leonardo3, 2008.

[11] J. Riskin, "Defecating Duck, or, the Ambigious Origins of Artificial Life," Critical Inquiry Journal, vol. 29, pp. 599-633, summer 2003.

[12] E. Thro, Robotics: The Marriage of Computers and Machines. Science \& Technology in Focus, Orient Longman Limited, 2003. 
[13] R. Cordeschi, The Discovery of the Artificial: Behavior, Mind and Machines Before and Beyond Cybernetics. Studies in Cognitive Systems, Springer, 2002.

[14] B. Miessner, Radiodynamics: The Wireless Control of Torpedoes and Other Mechanisms. New York D.Van Nostrand Company, 1916.

[15] T. Ramey, "PV-2D Harpoon 84062 (Tanker 101) comes back to life," 2010. http://www.twinbeech.com/images/Aircraft/manufacturers/ lockheed/PV-2/PV-2D84062/mx/cockpit/DSC08751web.jpg] [Online; accessed 9-April-2014].

[16] "Luftwaffe photo albums," 2012. http://www.throughtheireyes2.co. uk/. [Online; accessed 7-February-2014].

[17] L. Earnest, "Stanford Cart," 2012. http://www.stanford.edu/ learnest/cart.htm [Online; accessed 9-April-2014].

[18] W. Scheck, "Lawrence Sperry: Autopilot Inventor and Aviation Innovator," Aviation History Magazine Online, 2013. [Online; accessed 7February-2014].

[19] H. Wings, "George the Autopilot," Historic Wings: The Online Magazine of Aviators, Pilots and Adventures, 2012. http://fly.historicwings. com/2012/08/george-the-autopilot. [Online; accessed 9-April-2014].

[20] S. Nof, Springer Handbook of Automation. Springer Handbooks, Springer, 2009.

[21] K. P. Werrell and U. A. University, The Evolution of the Cruise Missile. Air University Press, 1985.

[22] "History of Sperry Marine." http: //www.sperrymarine.com/ corporate-history/sperry-marine. [Online; accessed 7-February2014].

[23] G. Jansson, "The V1 Missile or The Buzz Bomb," 2012. http://www. zenza.se/vw/.[Online; accessed 7-February-2014].

[24] Automatic Control Operational Sequence of the Transatlantic Flight:, "Importance of Landing," Flight and Aircraft Engineer, pp. 415-418, 1947. http://www .flightglobal.com.[Online; accessed 7-February-2014].

[25] S. Nof, Handbook of Industrial Robotics. No. v.1 in Electrical and electronic engineering, Wiley, 1999.

[26] J. L. Adams, Remote control with long transmission delays. PhD thesis, 
Mechanical Engineering, Stanford University, 1961.

[27] P. W. Braisted, Study of a predictor for remote control systems operating with signal transmission delays. PhD thesis, Mechanical Engineering, Stanford University, 1963.

[28] R. A. J. Schmidt, A study of the real-time control of a computer-driven vehicle. PhD thesis, Electrical Engineering, Stanford University, 1971.

[29] H. P. Moravec, Obstacle avoidance and navigation in the real world by a seeing robot rover. $\mathrm{PhD}$ thesis, Computer Science, Stanford University, 1980 .

[30] "Petman." http://www.bostondynamics.com/robot_petman.htm] [Online; accessed 9-April-2014].

[31] "ROV AUV." http://mars.jpl.nasa.gov/msl/ [Online; accessed 9April-2014].

[32] "Mars Science Laboratory Milestones." http://mars.jpl.nasa.gov/ msl/ [Online; accessed 9-April-2014].

[33] C. F. Tan, S. M. Liew, M. R. Alkahari, S. S. S. Ranjit, M. R. Said, W. Chen, M. Rauterberg, D. Sivakumar, and Sivarao, "Fire Fighting Mobile Robot: State of the Art and Recent Development," Australian Journal of Basic and Applied Sciences, vol. 7, no. 10, pp. 220-230, 2013.

[34] B. Yamauchi, "PackBot: A Versatile Platform for Military Robotics," in Proceedings of SPIE 5422, pp. 228-237, 2004.

[35] Y. Kageyama, "Toshiba shows four-legged robot for nuke disaster." Associate Press, Yokohama, Japan, November 2012. http://phys.org/news/ 2012-11-toshiba-four-legged-robot-nuke-disaster.html. [Online; accessed 10-February-2014].

[36] E. Ackerman, "Boston Dynamics Sand Flea Robot Demonstrates Astonishing Jumping Skills." IEEE Spectrum, March 2012. http://spectrum.ieee.org/automaton/robotics/military-robots/bostondynamics-sand-flea-demonstrates-astonishing-jumping -skills. [Online; accessed 10-February-2014].

[37] M. Raibert, K. Blankespoor, G. Nelson, R. Playter, and B. Team, "BigDogi the Rough-Terrain Quadruped Robot," in Proceedings of the 17th World Congress The International Federation of Automatic Control, pp. 1082210825, 2008.

[38] E. Guizzo, "Stunning Video of PETMAN Humanoid Robot 
From Boston Dynamics." IEEE Spectrum, October 2011. http://spectrum.ieee.org/automaton/robotics/humanoids/ stunning-video-of-boston-dynamics-petman-humanoid. [Online; accessed 10-February-2014].

[39] S. Cass, "DARPA Unveils Atlas DRC Robot." IEEE Spectrum, July 2013. http://spectrum.ieee.org/automaton/robotics/humanoids/ darpa-unveils-atlas-drc-robot. [Online; accessed 10-February-2014].

[40] E. Ackerman, "U.s. army considers replacing thousands of soldiers with robots." IEEE Spectrum, January 2014. http: //spectrum.ieee.org/automaton/robotics/military-robots/ army-considers-replacing-thousands-of-soldiers-with-robots. [Online; accessed 10-February-2014].

[41] E. Ackerman, "RHex Does Parkour All Over UPenn." IEEE Spectrum, July 2013. http://spectrum.ieee.org/automaton/robotics/ aerial-robots/rhex-does-parkour-all-over-upenn. [Online; accessed 10-February-2014].

[42] P. A. Avery, "Intelligent Vehicle Behavior and Emergent System Stability," International Journal of Automotive Engineering, 2011.

[43] M. Taylor and A. Wilby, "Design Considerations and Operational Advantages of a Modular AUV with Synthetic Aperture Sonar," in Proceedings of OCEANS 2011, (Waikoloa, Hawaii), pp. 1-6, 2011.

[44] F. Tobe, "Emerging Underwater Business: Three Companies to Note." The Robot Report, October 2011. http://www.everything-robotic. com/2011/10/emerging-underwater-businesses-three.html. [Online; accessed 11-February-2014].

[45] G. Lindberg, "Canadarm and its U of A Connections," U of A Engineer Magazine, University of Alberta, 2004.

[46] J. Gallentine, Ambassadors from Earth: Pioneering Explorations with Unmanned Spacecraft. Outward odyssey : a people's history of spaceflight, University of Nebraska Press, 2009.

[47] C. R. Frost, "Challenges and Opportunities for Autonomous Systems in Space," in Frontiers of Engineering: Reports on Leading-Edge Engineering from the 2010 Symposium, 2010.

[48] A. Mishkin, Sojourner: an insider's view of the Mars Pathfinder mission. Berkeley Books, 2003. http://books.google.com.tr/books?id= 9YFTAAAAMAAJ. [Online; accessed 10-February-2014]. 
[49] S. Squyres, Roving Mars: Spirit, Opportunity, and the Exploration of the Red Planet. Hyperion, 2005. http://books.google.com.tr/books?id= ebpSYlaoKOMC. [Online; accessed 10-February-2014].

[50] ESA, "European Robotic Arm," ESA Website, 2004.

[51] D. F. Blake, R. V. Morris, G. Kocurek et al., "Curiosity at Gale Crater, Mars: Characterization and Analysis of the Rocknest Sand Shadow," Science, vol. 341, pp. 220-230, September 2013.

[52] "Task Force Report: The Role of Autonomy in DoD Systems," 2012.

[53] "Delivery my parcel using a drone? just kidding, isn't it?," 2013. http: //endd122.net/wordpress/?cat=640 [Online; accessed 9-April-2014].

[54] "Testing Unmanned Helicopters for Pesticide Spraying in California," 2013. http://www.uasvision.com/2013/06/10/testing-unmannedhelicopters-for-pesticide-spraying-in-california [Online; accessed 9-April2014].

[55] "Drone ZALA 421-16E," 2012. http://zala .aero/zala-421-16e-2 [Online; accessed 9-April-2014].

[56] "B.C. Cops Mulling the Use of UAV's in Major Crash Scenes," 2011. http: //novus2.com/uav360/2011/08/b-c-cops-mulling-the-use-of-uav\% E2\%80\%99s-in-major-crash-scenes [Online; accessed 9-April-2014].

[57] "The USN's Rent-A-UAV Contract, 2012-2017," 2012. http://www.defenseindustrydaily.com/ the-usns-uas-isr-contract-2012-2017-07322/] [Online; accessed 9-April-2014].

[58] "Solara 50," 2012. http://titanaerospace.com/platforms/ solara-50/\# [Online; accessed 9-April-2014].

[59] "Helios Prototype," 2012. http://www.nasa.gov/centers/dryden/ news/ResearchUpdate/Helios/ [Online; accessed 9-April-2014].

[60] "Harvard Microrobotics Lab." http://micro.seas.harvard.edu/ [Online; accessed 9-April-2014].

[61] "South Korea Developing VTOL Unmanned Aerial Vehicle," 2011. http://www.unmanned.co.uk/ unmanned-vehicles-news/unmanned-aerial-vehicles-uav-news/ south-korea-developing-vtol-unmanned-aerial-vehicle/ [Online; accessed 9-April-2014]. 
[62] "Agriculture the New Game of Drones," 2012. http://www. unmannedsystemsaustralia.com.au/latestnews/?p=831 [Online; accessed 9-April-2014].

[63] "Gull UAV: Introduction," 2012. http://www.warrioraero.com/GULL/ [Online; accessed 9-April-2014].

[64] J. Horgan, "Unmanned Flight," 2013. ngm.nationalgeographic. com/2013/03/unmanned-flight/horgan-text [Online; accessed 9-April2014].

[65] "Phantom Eye (HALE) ") 2012. http://www. boeing.com/boeing/bds/ phantom_works/phantom_eye.page [Online; accessed 9-April-2014].

[66] C. Cole, "Drone Wars A Briefing Document," NEXUS New Times Magazine, vol. 19, no. 3, pp. 11-16, 2012.

[67] "The MQ-8B Fire Scout, a robotic spy chopper," 2012. http://www. latimes.com/business/la-fi-0411-drone-chopper-pictures, 0,3940595 . photogallery? index $=$ lat-firescout m2a0s7pd20120410134627 [Online; accessed 9-April-2014].

[68] T. Sheridan, Telerobotics, Automation, and Human Supervisory Control. MIT Press, 1992.

[69] R. Parasuman, T. Sheridan, and C. Wickens, "A Model for Types and Levels of Human Interaction with Automation," IEEE Transactions on Systems, Man and Cybernetics- Part A: Systems and Humans, vol. 30, pp. 286-297, May 2000.

[70] A. Turing, "Computing Machinery and Intelliegence," Mind, vol. LIX (236), pp. 433-460, 1950.

[71] B. Hasslacher and M. Tilden, "Living Machines," in IN IEEE WORKSHOP ON BIO-MECHATRONICS, L. STEELS, pp. 143-169, 1996.

[72] M. Cleary, M. Abramson, M. Adams, and S. Kolitz, "Metrics for Embedded Collaborative Intelligent Systems," in PERMIS 2000: Performance Metrics for Intelligent Systems Workshop, 2000.

[73] J. Boyd., "The Essence of Winning and Losing," 1995. http://www. danford.net/boyd/essence.htm [Online; accessed 29-March-2014].

[74] H. M. Huang, K. Pavek, B. Novak, J. Albus, and E. Messina, "A Framework For Autonomy Levels For Unmanned Systems (ALFUS)," 2007.

[75] F. Kendoul, "Survey of Advances in Guidance, Navigation and Control of 
Unmanned Rotorcraft Systems," Journal of Field Robotics, vol. 29, no. 2, pp. 315-378, 2012.

[76] W. Johnson, Helicopter Theory. Dover Books on Aeronautical Engineering, Dover Publications, 2012.

[77] J. G. Leishman, Principles of Helicopter Aerodynamics with CD Extra. Cambridge Aerospace Series, Cambridge University Press, 2006.

[78] A. E. Moore, "Will the Helicopter Become the Future Flying Machine?," Popular Aviation, pp. 8-10, January 1934.

[79] B. Petite, "The Weird and Wacky." Vertical Magazine, January 2012. http://www.verticalmag.com/features/features_article/ the-weird-and-wacky.html\#.U2zT-fl_tH4. [Online; accessed 9-May2014].

[80] V. Miklos, "A Treasury of Flying Cars from the Golden Age of Aviation." iO9, March 2013. http://io9.com/ a-treasury-of-flying-cars-from-the-golden-age-of-aviat-51091. [Online; accessed 9-May-2014].

[81] L. R. G. Carrillo, A. E. D. Lopez, R. Lozano, and C. Pégard, Quad Rotorcraft Control: Vision-Based Hovering and Navigation. Advances in Industrial Control, Springer London, Limited, 2012.

[82] P. Pounds, R. Mahony, and P. Corke, "Modelling and Control of a QuadRotor Robot," in Proceedings of the Australiasian Conf. On Robotics and Automation, (Auckland, New Zealand), December 2006.

[83] S. Bouabdallah, A. Noth, and R. Siegwart, "PID vs LQ control Techniques Applied to an Indoor Micro Quadrotor," in Proceedings of 2004 IEEE/RSJ International Conference on Intelligent Robots and Systems (IROS 2004), vol. 3, (Sendai, Japan), pp. 2451-2456, October 2004.

[84] A. Mokhtari, A. Benallegue, and B. Daachi, "Robust Feedback Linearization and $G H_{\text {inf }}$ Controller for a Quadrotor Unmanned Aerial Vehicle," in Proceedings of the 12th IFAC World Congress, (Sydney, Australia), pp. 251-254, 1993.

[85] G. M. Hoffmann, S. L. Waslander, and C. J. Tomlin, "Quadrotor helicopter trajectory tracking control," in Proceedings of AIAA Guidance, Navigation, and Control Conference, 2008.

[86] G. M. Hoffmann, H. Huang, S. L. Waslander, and C. J. Tomlin, "Quadrotor Helicopter flight dynamics and control: Theory and experiment," in Proceedings of AIAA Guidance, Navigation, and Control Conference, 2007. 
[87] J. H. Gillula, G. M. Hoffmann, H. Huang, M. P. Vitus, and C. J. Tomlin, "Applications of hybrid reachability analysis to robotic aerial vehicles," International Journal of Robotic Research, vol. 30, no. 3, pp. 335-354, 2011.

[88] D. Mellinger and V. Kumar, "Minimum snap trajectory generation and control for quadrotors," in Proceedings of 2011 IEEE International Conference on Robotics and Automation (ICRA), (Shanghai, China), pp. 2520$2525,2011$.

[89] A. Kushleyev, V. Kumar, and D. Mellinger, "Towards A Swarm of Agile Micro Quadrotors," in Proceedings of Robotics: Science and Systems, (Sydney, Australia), July 2012.

[90] D. Mellinger, M. Shomin, and V. Kumar, "Control of Quadrotors for Robust Perching and Landing," in Proceedings of the International Powered Lift Conference, Oct 2010.

[91] D. Mellinger, Q. Lindsey, M. Shomin, and V. Kumar, "Design, Modeling, Estimation and Control for Aerial Grasping and Manipulation," in Proceedings of the IEEE International Conference on Intelligent Robots and Systems (IROS), Sept 2011.

[92] F. Kendoul, Z. Yu, and K. Nonami, "Guidance and Nonlinear Control System for Autonomous Flight of Mini-rotorcraft Unmanned Aerial Vehicles," Journal of Field Robotics, vol. 27, no. 3, pp. 311-334, 2010.

[93] F. Kendoul, I. Fantoni, and K. Nonami, "Optic Flow-Based Vision System for Autonomous 3D Localization and Control of Small Aerial Vehicles," Robotics and Autonomous Systems, vol. 57, no. 6, pp. 591-602, 2009.

[94] C. Nicol, C. J. B. Macnab, and A. Ramirez-Serrano, "Adaptive fuzzy control for a quadrotor helicopter robust to wind buffeting," in Canadian Conference on Electrical and Computer Engineering, CCECE 2008, pp. 1233-1238, 2008.

[95] F. Kendoul, K. Nonami, I. Fantoni, and R. Lozano, "An adaptive visionbased autopilot for mini flying machines guidance, navigation and control," Autonomous Robots, vol. 27, no. 3, pp. 165-188, 2009.

[96] G. V. Raffo, M. G. Ortega, and F. R. Rubio, "An integral predictive/nonlinear $H_{i} n f$ control structure for a quadrotor helicopter," Automatica, vol. 46, pp. 29-39, 2010.

[97] E. Altug, J. P. Ostrowski, and C. J. Taylor, "Control of a Quadrotor Helicopter Using Dual Camera Visual Feedback," International Journal of 
Robotics Research, vol. 24, no. 5, pp. 329-341, 2005.

[98] N. Guenard, T. Hamel, and R. E. Mahony, "A practical Visual Servo Control for a Unmanned Aerial Vehicle," in Proceedings of 2007 IEEE International Conference on Robotics and Automation (ICRA), pp. 13421348, IEEE, 2007.

[99] S. Bouabdallah and R. Siegwart, "Backstepping and Sliding-Mode Techniques Applied to an Indoor Micro Quadrotor," in Proceedings of 2005 IEEE International Conference on Robotics and Automation (ICRA), (Barcelona, Spain), pp. 2247-2252, IEEE, April 2005.

[100] S. Bouabdallah and R. Siegwart, "Full Control of a Quadrotor," in Proceedings of 2007 IEEE/RSJ International Conference on Intelligent Robots and Systems (IROS 200\%, (San Diego, CA, USA), pp. 153-158, October 2007.

[101] E. Johnson and S. Kannan, "Adaptive trajectory control for autonomous helicopters," AIAA Journal of Guidance, Control, and Dynamics, vol. 28, no. 3 , p. $524-538,2005$.

[102] J. Wang, T. Bierling, L. Höcht, F. Holzapfel, S. Klose, and A. Knoll, "Novel Dynamic Inversion Architecture Design for Quadrocopter Control," Advances in Aerospace Guidance, Navigation and Control, pp. 261-272, 2011.

[103] P. Castillo, A. Dzul, and R. Lozano, "Real-time stabilization and tracking of a four rotor mini-rotorcraft," IEEE Transactions on Control Systems Technology, vol. 12, no. 4, p. 510-516, 2004.

[104] P. Castillo, P. Albertos, P. Garcia, and R. Lozano, "Simple Real Time Attitude AStabilziation of a Quadrotor Aircraft with Bounded Signals," in Proceedings of 45th IEEE Conference on Decision and Control, pp. 15331539, December 2006.

[105] A. Benallegue, A. Mokhtari, and L. Fridman, "Feedback Linearization and High Order Sliding Mode Observer for a Quadrotor UAV," in Proceedings of the 2006 International Workshop on Variable Structure Systems, (Alghero, Italy), pp. 365-372, June 2006.

[106] T. Lee, M. Leoky, and N. H. McClamroch, "Nonlinear Robust Tracking Control of a Quadrotor UAV on SE(3)," Asian Journal of Control, vol. 15, pp. 391-408, March 2013.

[107] T. Lee, M. Leoky, and N. H. McClamroch, "Control of Complex Maneuvers for a Quadrotor UAV using Geometric Methods on SE(3)," arxiv.org, 2011. 
[108] J. F. Montgomery and G. A. Bekey, "Learning Helicopter Control Through Teaching by Showing," in Proceedings of the 37th IEEE Conference on Decision and Control, vol. 4, (Florida, USA), pp. 3647-3652, 1998.

[109] M. Sugeno, I. Hirano, and S. Kotsu, "Intelligent Control of an Unmanned Helicopter Based on Fuzzy Logic," in Proceedings of the 51st American Helicopter Society (AHS) Annual Forum, vol. 1, (Fort Worth, Texas, USA), p. 791-803, May 1998.

[110] C. Coza and C. J. B. Macnab, "A New Robust Adaptive-Fuzzy Control Method Applied to Quadrotor Helicopter Stabilization," in Proceedings of the Annual meeting of the North American Fuzzy Information Processing Society, (NAFIPS 2006), (Montreal, Quebec), pp. 454-458, 2006.

[111] M. Achtelik, A. Bachrach, R. He, S. Prentice, and N. Roy, "Stereo vision and laser odometry for autonomous helicopters in GPS-denied indoor environments," in Proceedings of SPIE7332, Unmanned System Technology XI, 733219, April 2009.

[112] S. Ahrens, D. Levine, G. Andrews, and J. P. How, "Vision-based Guidance and Control of a Hovering Vehicle in Unknown, GPS-denied Environments," in Proceedings. of IEEE International Conference on Robotics and Automation (ICRA), pp. 2643-2648, May 2009.

[113] N. Guenard, T. Hamel, and R. Mahony, "A practical visual servo control for an unmanned aerial vehicle," IEEE Transactions on Robotics, vol. 24, no. 2, pp. 331-340, 2008.

[114] J. Courbon, Y. Mezouar, N. Guenard, and P. Martinet, "Vision-based navigation of unmanned aerial vehicles," Control Engineering Practice, vol. 18, pp. 789-799, 2010.

[115] J. Conroy, G. Gremillion, B. Ranganathan, and J. S. Humbert, "Implementation of wide-field integration of optic flow for autonomous quadrotor navigation," Autonomous Robots, vol. 27, pp. 189-198, 2009.

[116] B. Bethke, M. Valenti, and J. P. How, "Cooperative vision based estimation and tracking using multiple UAVs," in Proceedings of the 7th International Conference on Cooperative Control and Optimization, vol. 369, (Gainesville,FL), pp. 179-189, Jan-Feb 2007.

[117] A. Bachrach, R. He, and N. Roy, "Autonomous flight in unknown indoor environments," International Journal of Micro Aerial Vehicles, vol. 1, no. 4, pp. 217-228, 2009.

[118] R. He, S. Prentice, and N. Roy, "Planning information Space for a quadro- 
tor helicopter in a GPS-denied environment," in Proceedings of IEEE International Conference on Robotics and Automation, (Pasadena,CA), pp. 1814-1820, 2008.

[119] K. Culligan, M. Valenti, Y. Kuwata, and J. P. How, "Three-dimensional flight experiments using on-line mixed-integer linear programming trajectory optimization," in Proceedings of IEEE American Control Conference, (New York), pp. 5322-5327, 2007.

[120] R. He, A. Bachrach, and N. Roy, "Efficient planning under uncertainty for a target-tracking micro aerial vehicle," in Proceedings of IEEE International Conference on Robotics and Automation, (Anchorage, AK), May 2010 .

[121] G. M. Hoffmann, S. L. Waslander, M. Vitus, H. Gillula, V. Pradeep, and C. J. Tomlin, "Stanford testbed of autonomous rotorcraft for multi-agent control," in Proceedings of the IEEE/RSJ International Conference on Intelligent Robots and Systems, (St. Louis,MO), pp. 404-405, 2009.

[122] N. Michael, J. Fink, and V. Kumar, "Cooperative manipulation and transportation with aerial robots," Autonomous Robots, vol. 30, no. 1, p. 73-86, 2011.

[123] H. L. Choi, L. Brunet, and J. P. How, "Consensus-based decentralized auctions for robust task allocation," IEEE Transactions on Robotics, vol. 25, no. 4, pp. 912-926, 2009.

[124] M. Cutler and J. P. How, "Actuator Constrained Trajectory Generation and Control for Variable-Pitch Quadrotors," in Proceedings of AIAA Guidance, Navigation, and Control Conference, (Minneapolis, Minnesota), August 2012.

[125] T. Toksoz, J. Redding, M. Michini, B. Michini, J. P. How, M. Vavrina, and J. Vian, "Automated Battery Swap and Recharge to Enable Persistent UAV Missions," in AIAA Infotech@Aerospace Conference, March 2011. (AIAA-2011-1405).

[126] M. Hehn, R. Ritz, and R. D'Andrea, "Performance Benchmarking of Quadrotor Systems Using Time-Optimal Control," Autonomous Robots, vol. 33, no. 1-2, pp. 69-88, 2012.

[127] M. Mueller, M. Hehn, and R. D’Andrea, “A Computationally Efficient Algorithm for State-to-State Quadrocopter Trajetory Generation and Feasiblity Verification," in Proceedings of 2013 IEEE/RSJ International Conference on Intelligent Robots and Systems, (Tokyo, Japan), November 2013. 
[128] A. Schoellig, F. Mueller, and R. D'Andrea, "Optimization-based iterative learning for precise quadrocopter trajectory tracking," Autonomous Robots, vol. 33, no. 1-2, pp. 103-127, 2012.

[129] E. Yilmaz and A. T. Kutay, "The Simulation of Attitude Controller Design for a Quadrotor Model via Several Methods from Literature," in Proceedings of the 2013 AIAC Ankara International Aerospace Conference, (Ankara, Turkey), September 2013.

[130] M. Tooley, A. Filippone, T. H. G. Megson, M. V. Cook, P. W. Carpenter, E. L. Houghton, D. Wyatt, L. R. Jenkinson, J. Marchman, F. D. Florio, et al., Aerospace Engineering e-Mega Reference. Elsevier Science, 2009.

[131] L. Pivano, Thrust Estimation and Control of Marine Propellers in FourQuadrant Operations. PhD thesis, Norwegian University of Science and Technology, 2008.

[132] R. Nelson, Flight Stability and Automatic Control. McGraw-Hill, October 1997.

[133] M. Achtelik, "Non-linear and Adaptive Control of a Quadcopter. Diplomarbeit," July 2010.

[134] F. Holzapfel, Non-linear and Adaptive Control of a UAV. PhD thesis, Technische Universitat Munchen, 2004.

[135] J. J. E. Slotine and W. A. Li, Applied Nonlinear Control. Englewood Cliffs, NJ: Prentice Hall, 1991.

[136] H. K. Khalil, Nonlinear Systems. Upper Saddle River, NJ: Prentice Hall, 1996.

[137] A. Isidori, Nonlinear Control Systems. London, UK: Springer, 2001.

[138] E. N. Johnson and A. J. Calise, "Pseudo-Control Hedging: A New Method For Adaptive Control," in Proceedings of the Advances in Navigation Guidance and Control Technology Workshop, (Alabama, USA), 2000.

[139] P. Iannou and B. Fidan, Adaptive Control Tutorial: Advances in Design and Control. Phialdelphia, US: Society for Industrial and Applied Mathematics, 2006.

[140] C. Grant, "Lyapunov's Direct Method," Lecture Notes of Mathematics 634-2, 1999.

[141] K. S. Narendra and A. M. Annaswamy, Stable Adaptive Systems. Englewwod Cliffs, N.J: Dover Publications Inc., 1989. 
[142] I. Kanellakopoulos and P. Krein, "Integral-Action Nonlinear Control of Induction Motors," in Proceedings of the 12th IFAC World Congress, (Sydney, Australia), pp. 251-254, 1993.

[143] Y. Tan, J. Chang, J. Hu, and H. Tan, "Advanced Nonlinear Control Strategy for Motion Control Systems," in Proceedings of the International Power Electronics and Motion Control Conference, vol. 1, (Beijing, China), pp. 161-121, August 2000. 


\section{APPENDIX A}

\section{FURTHER CONSTANT TRAJECTORY RESULTS}

In this section, the results for various simulation cases can be investigated further. For the discussion of these figures, refer to section 4.2

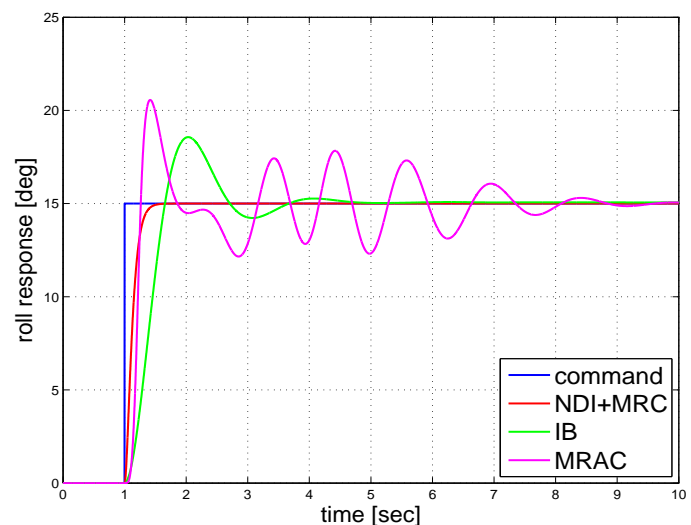

(a) Roll State Response

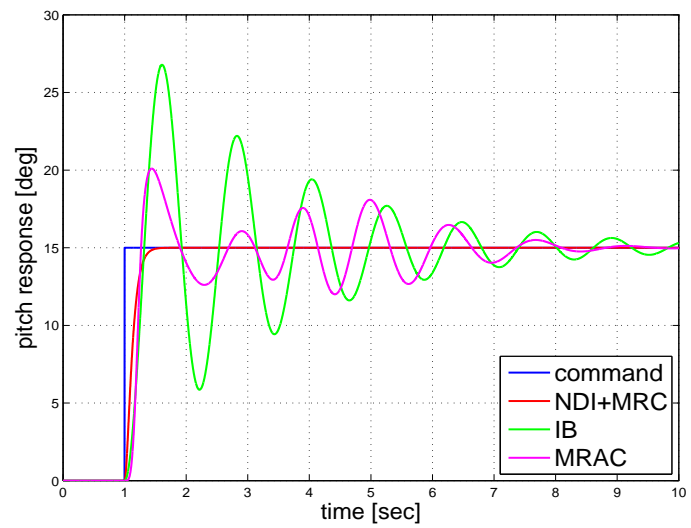

(c) Pitch State Response

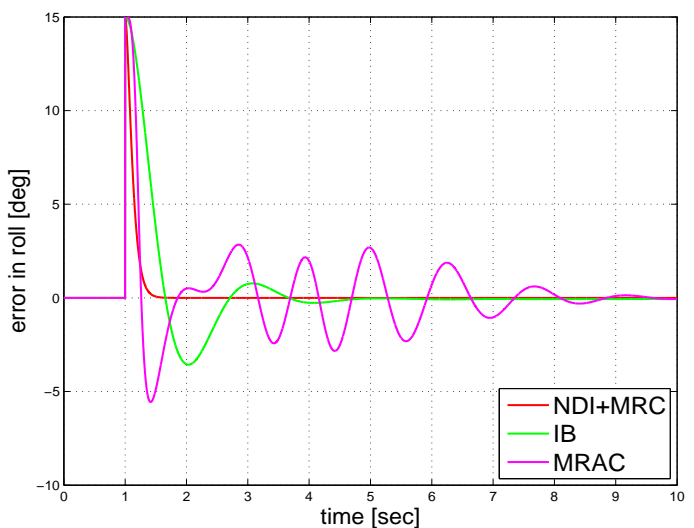

(b) Roll Error

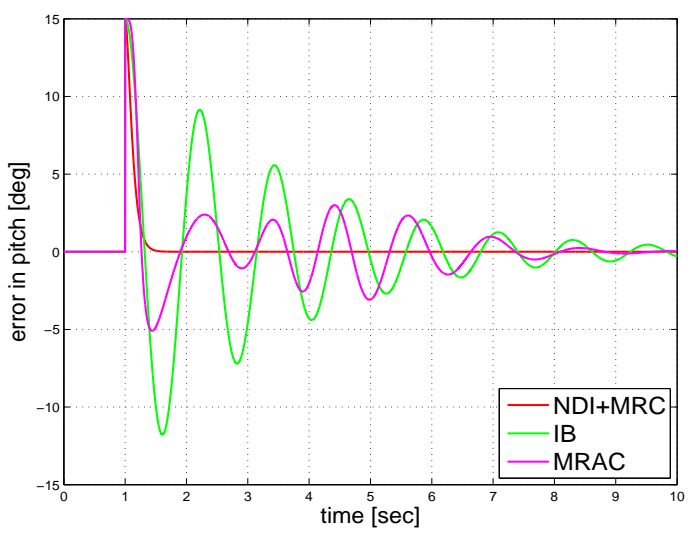

(d) Pitch Error

Figure A.1: Simulation of angular control for fixed angular orientation tracking in two axes with no noise 


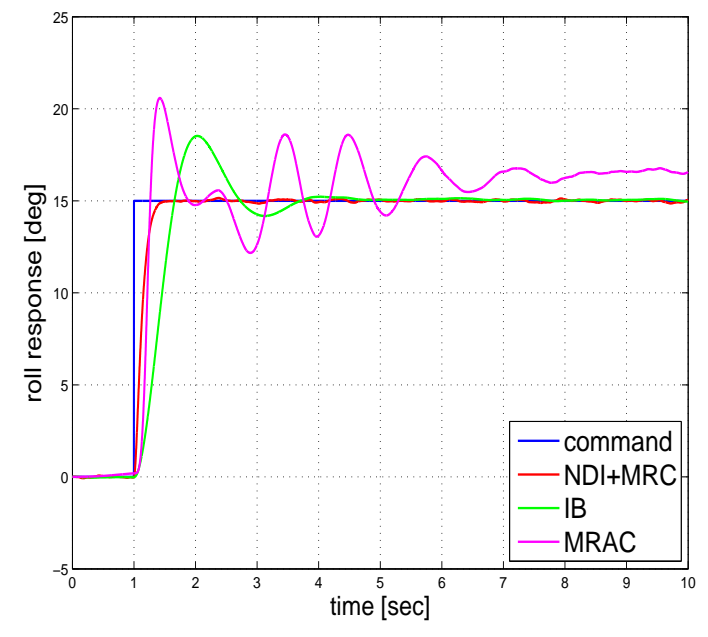

(a) Roll State Response

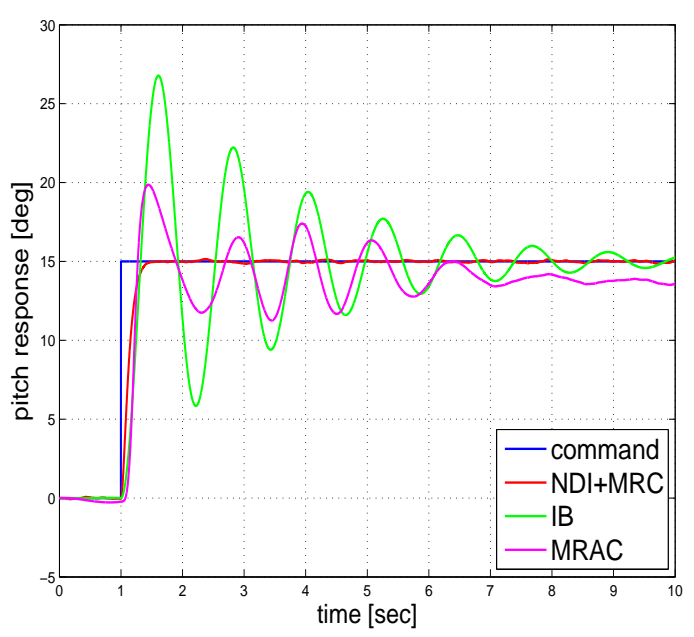

(c) Pitch State Response

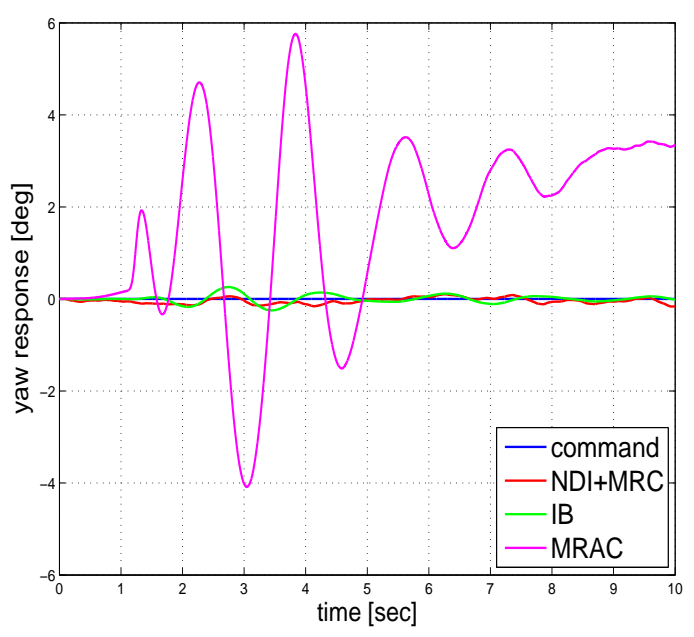

(e) Yaw State Response

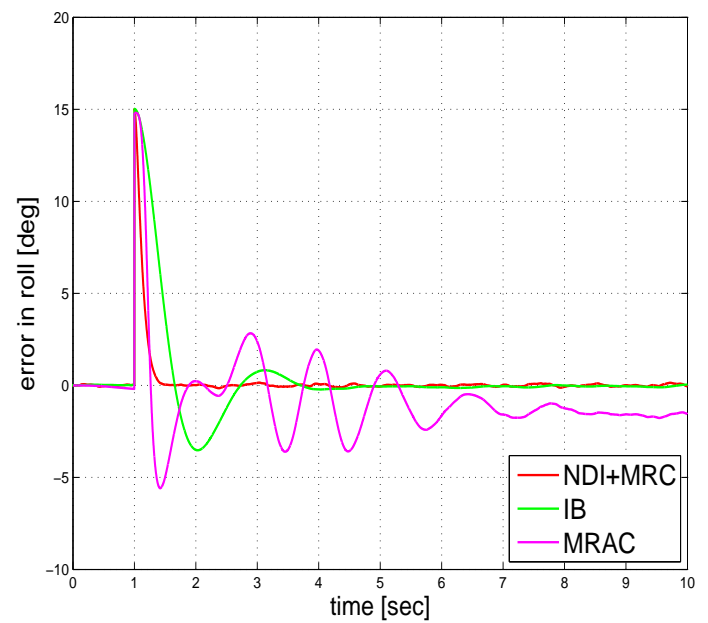

(b) Roll Error

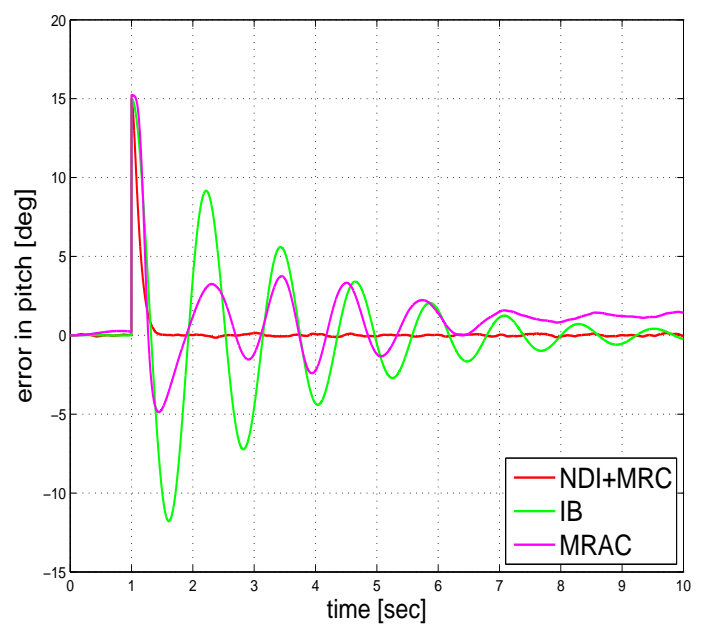

(d) Pitch Error

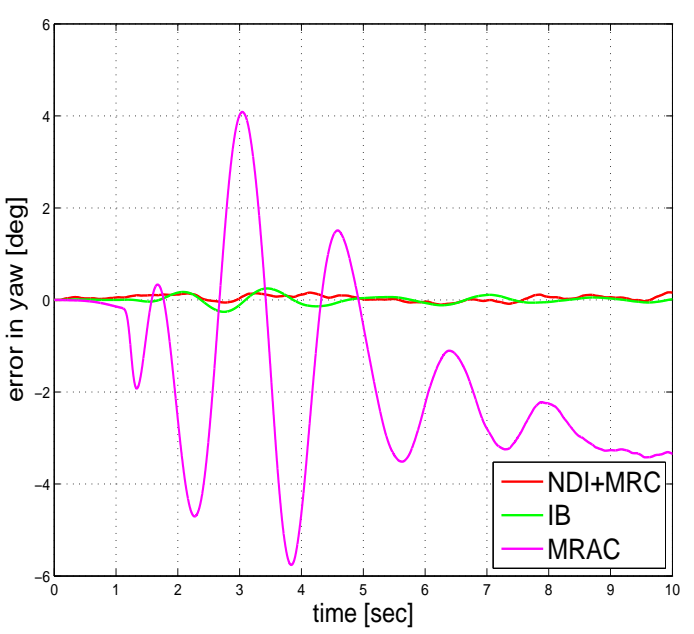

(f) Yaw Error

Figure A.2: Simulation of angular control for sinusoidal tracking in two axes with noise addition 


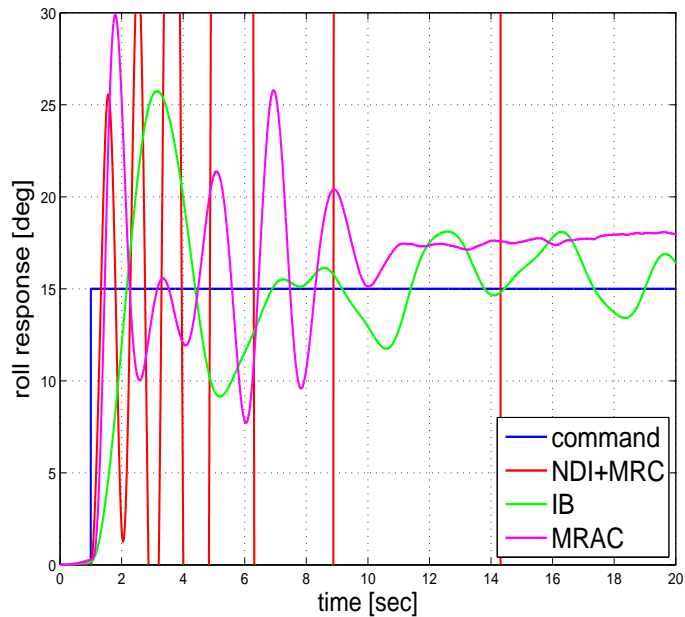

(a) Roll State Response

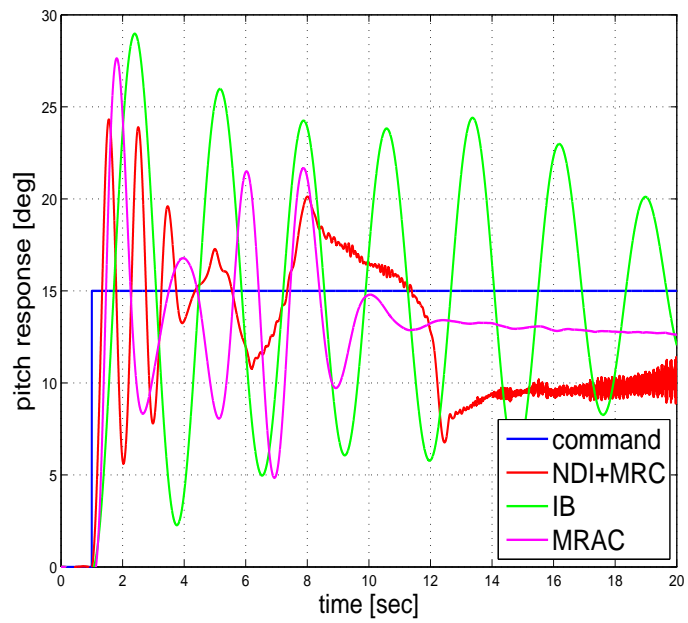

(c) Pitch State Response

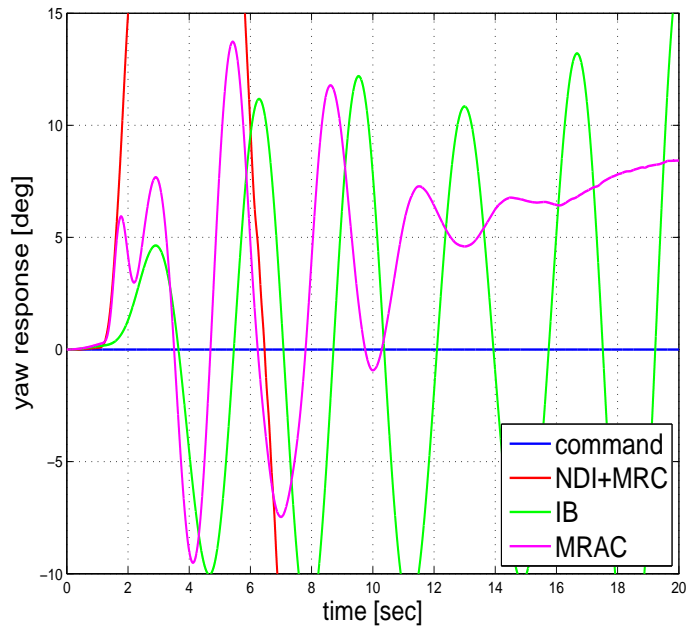

(e) Yaw State Response

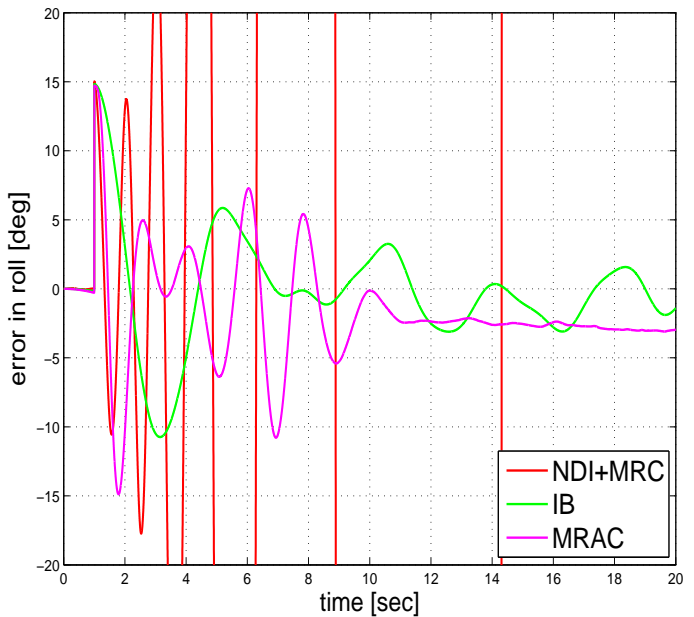

(b) Roll Error

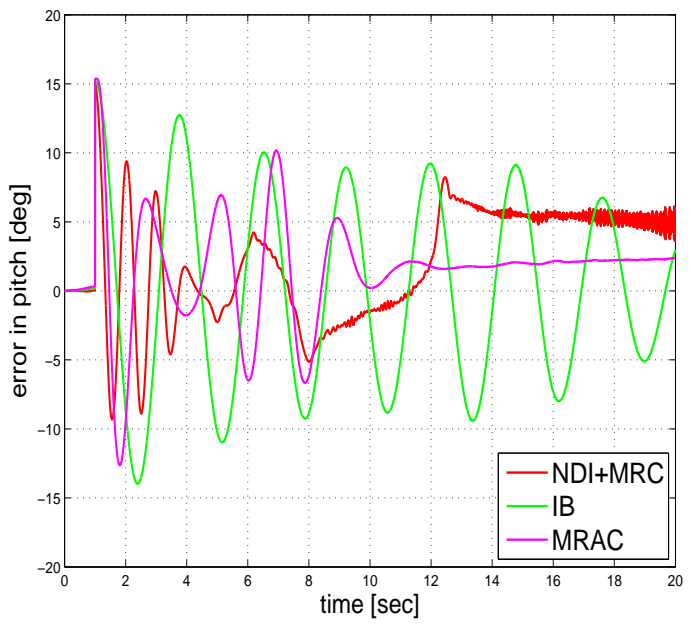

(d) Pitch Error

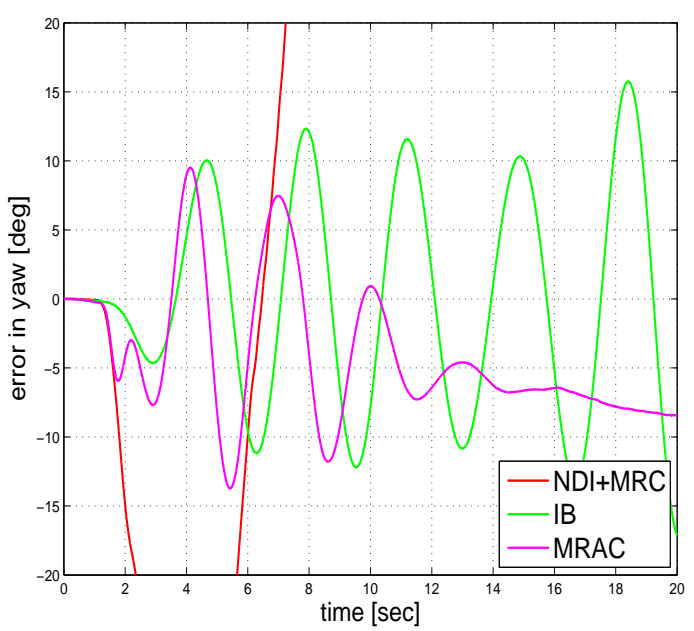

(f) Yaw Error

Figure A.3: Simulation of angular control for sinusoidal tracking in two axes with a different quadrotor model 


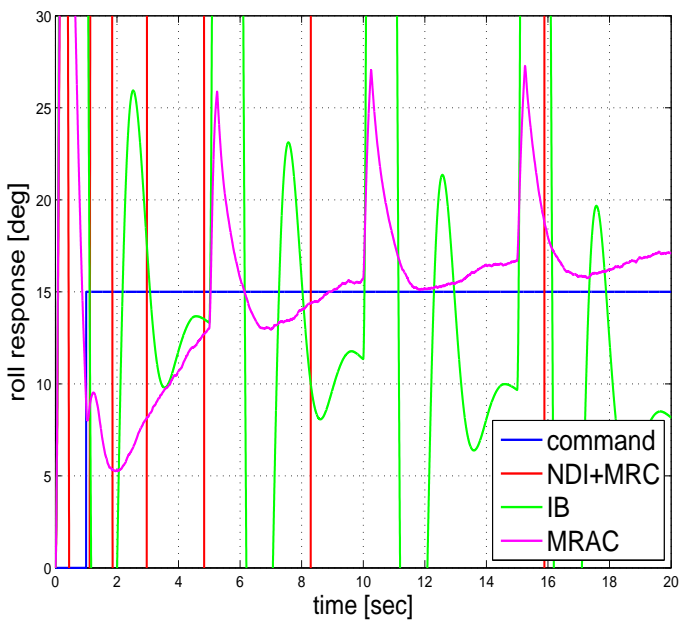

(a) Roll State Response

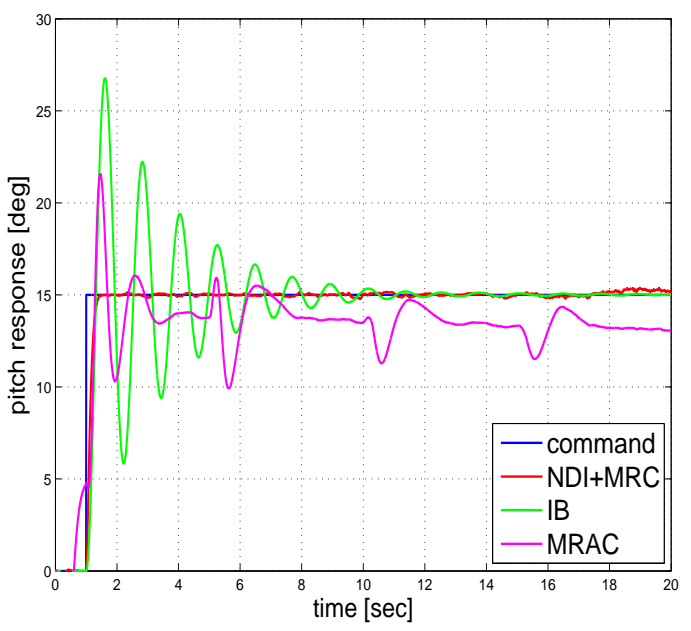

(c) Pitch State Response

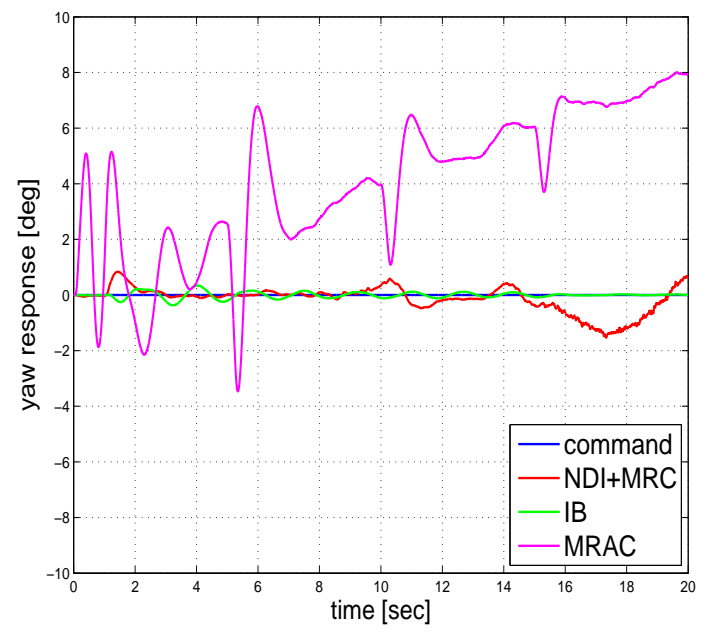

(e) Yaw State Response

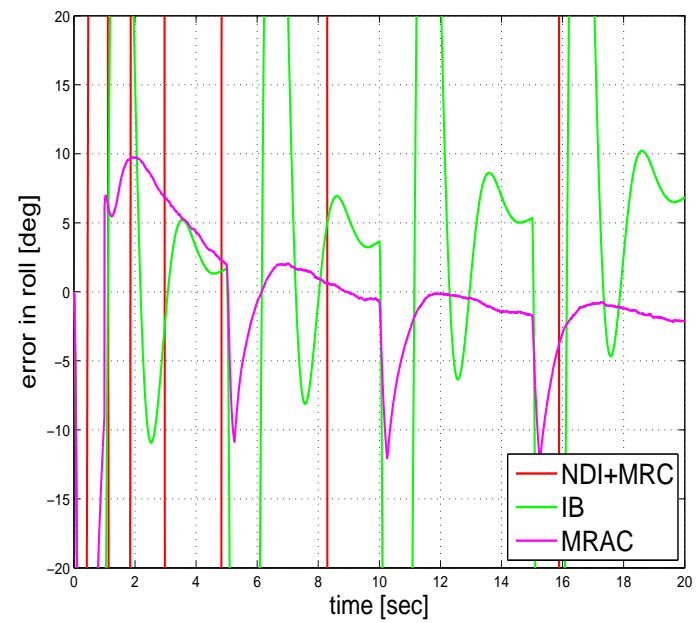

(b) Roll Error

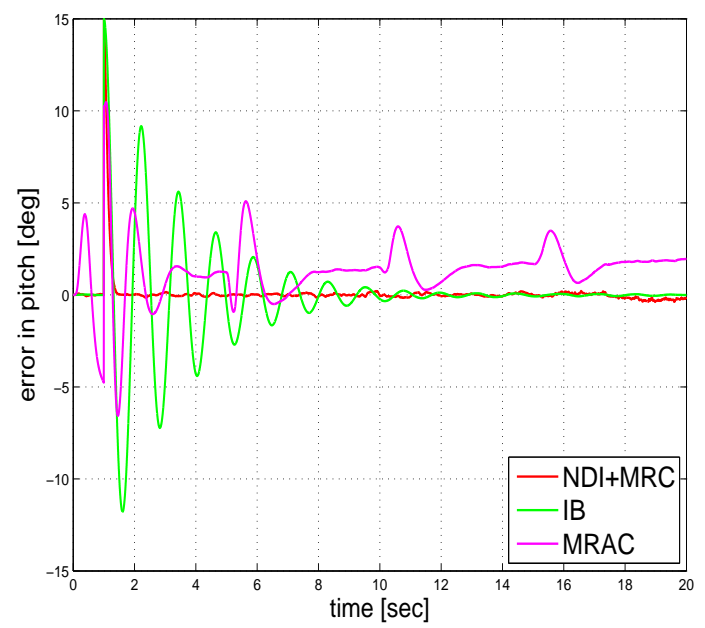

(d) Pitch Error

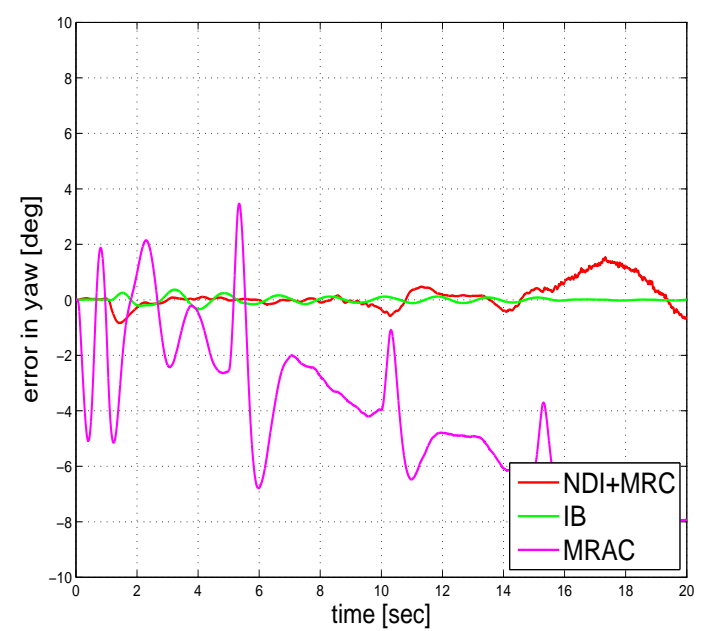

(f) Yaw Error

Figure A.4: Simulation of angular control for sinusoidal tracking in two axes in the presence of unknown disturbance 


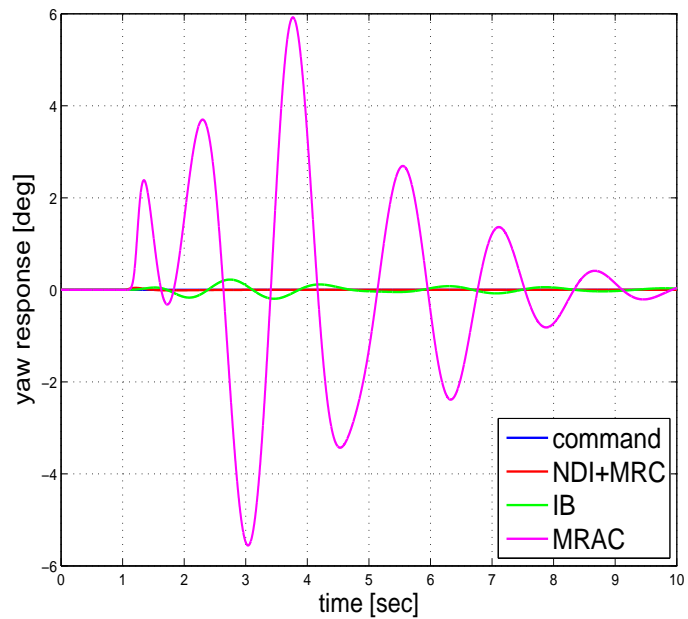

(a) Yaw State Response

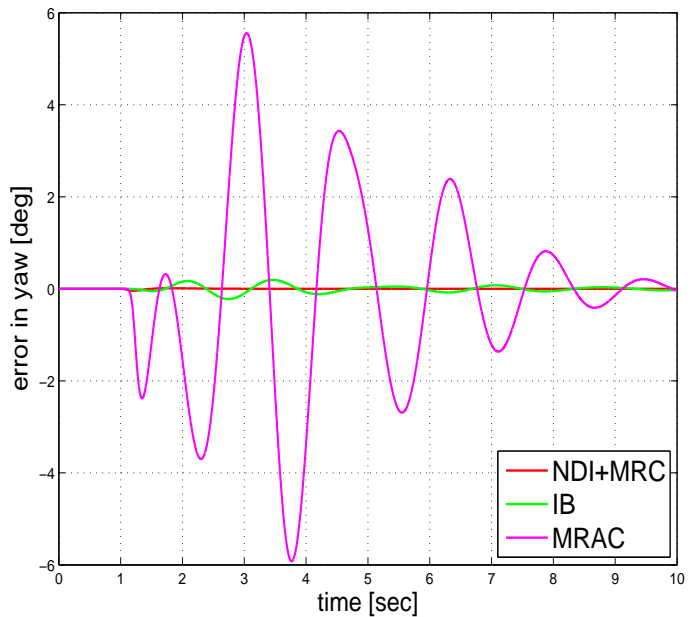

(b) Yaw Error

Figure A.5: Simulation of angular control for fixed angular orientation tracking in two axes with no noise (cont.) 


\section{APPENDIX B}

\section{FURTHER SINUSOIDAL INPUT RESULTS}

For the discussion of the figures in this appendix, refer to Section 4.3 .
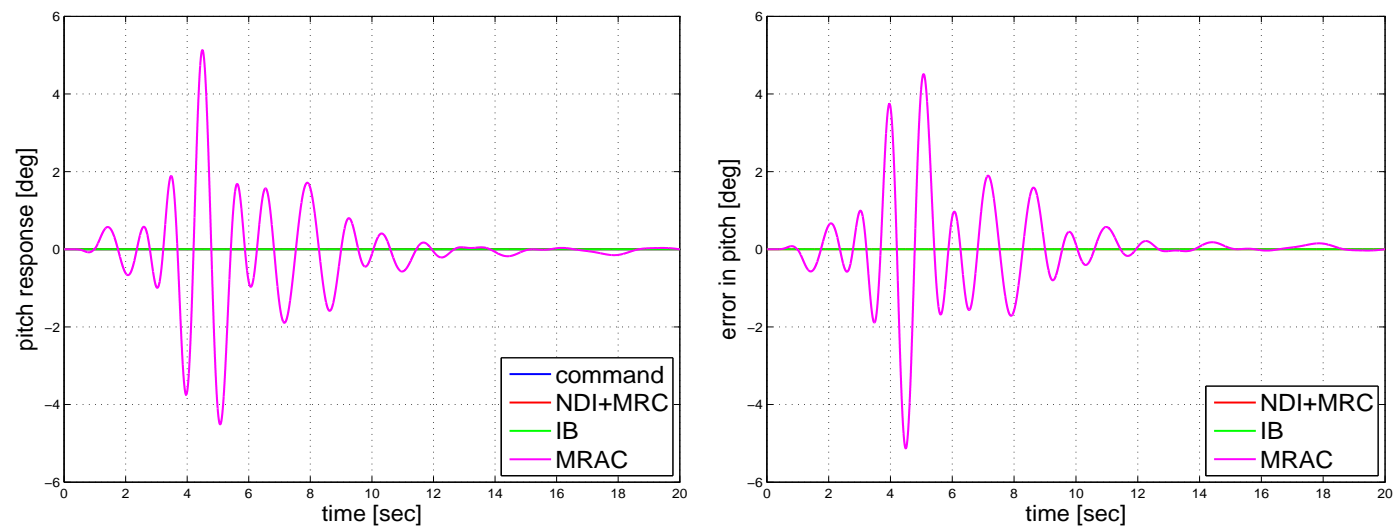

(a) Pitch Error

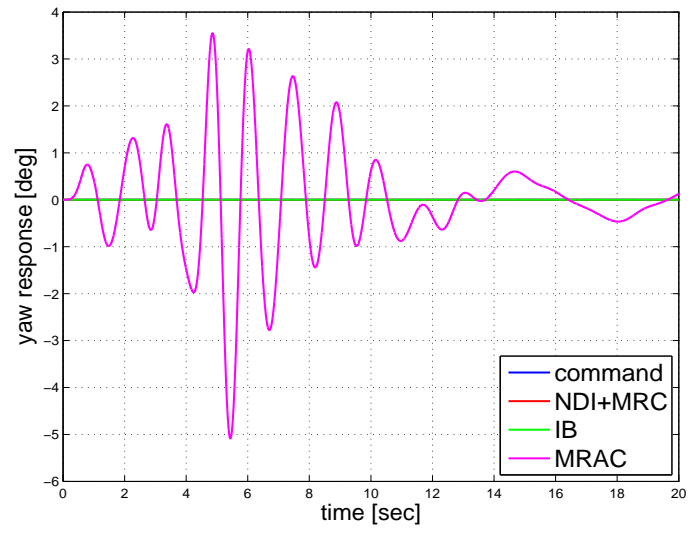

(b) Yaw State Response

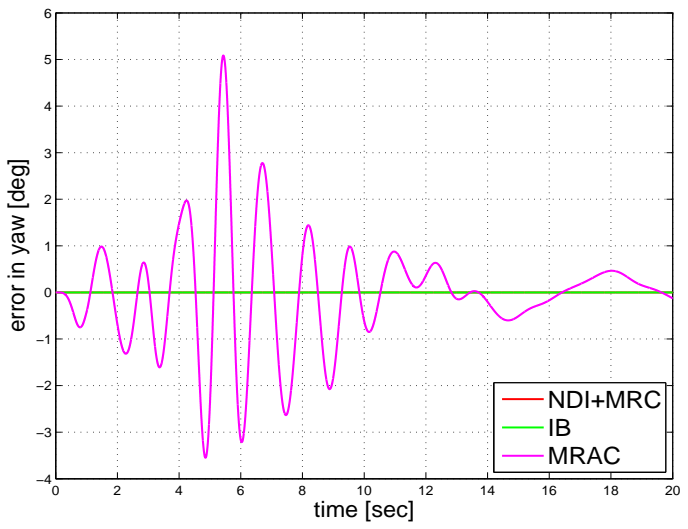

(c) Yaw Error

Figure B.1: Simulation of angular control for sinusoidal tracking with no noise 


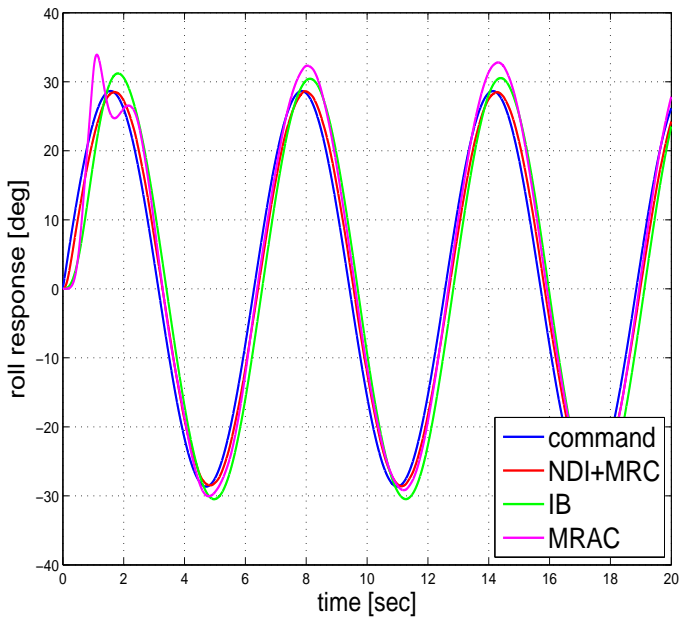

(a) Roll State Response

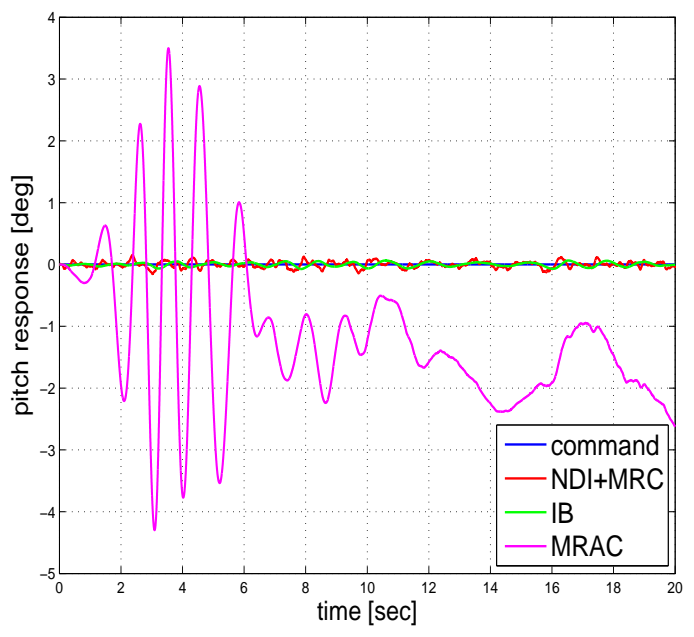

(c) Pitch State Response

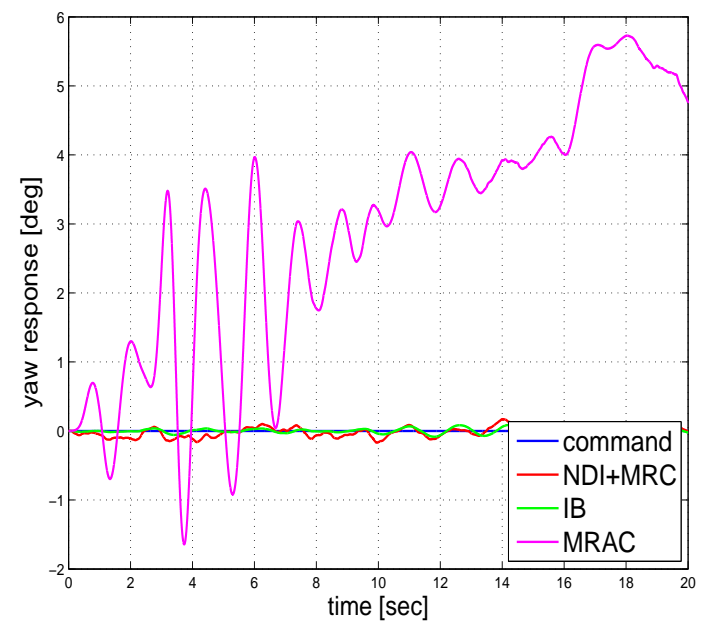

(e) Yaw State Response

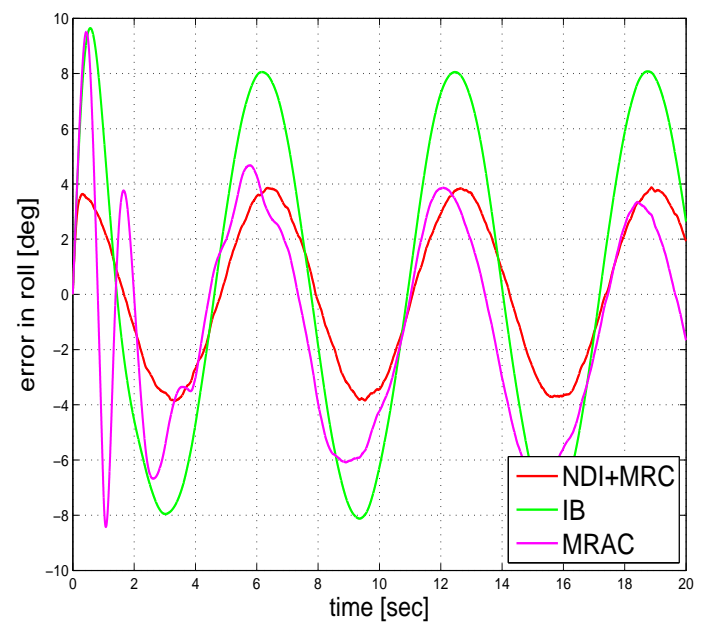

(b) Roll Error

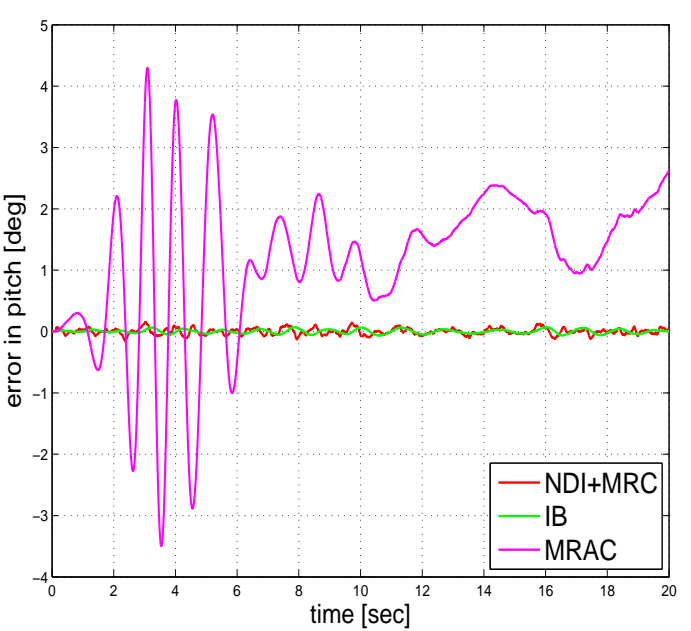

(d) Pitch Error

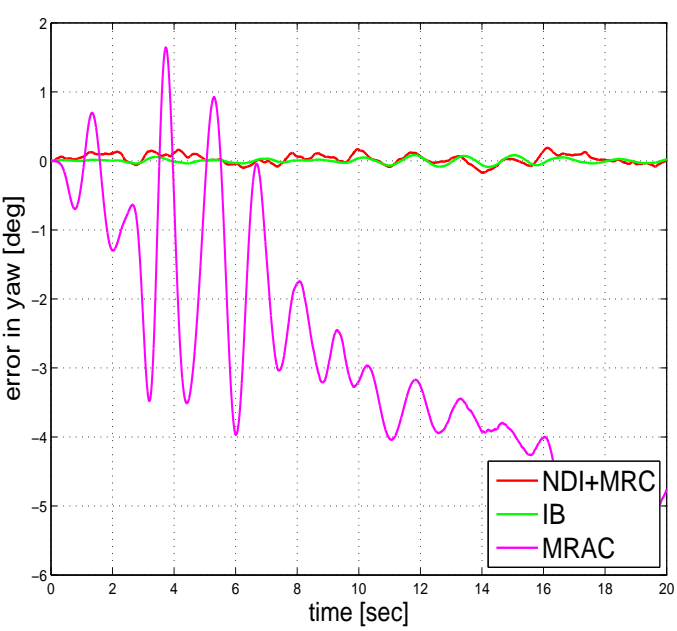

(f) Yaw Error

Figure B.2: Simulation of angular control for sinusoidal tracking with noise addition 


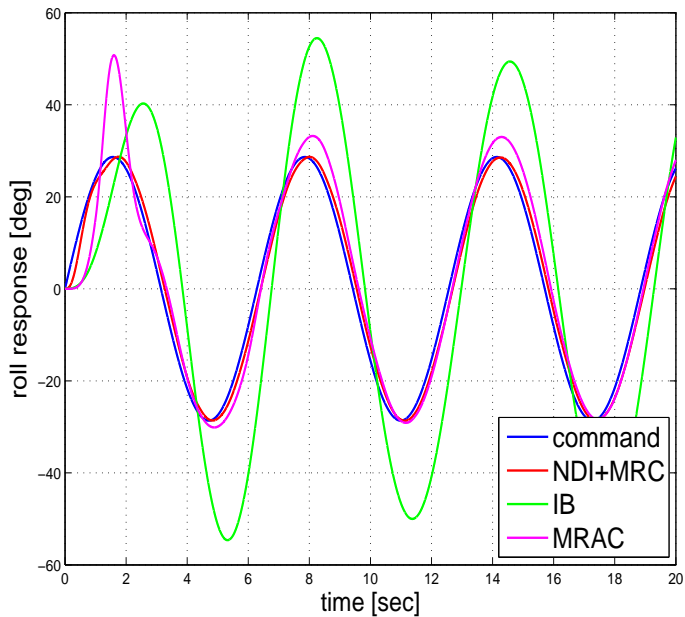

(a) Roll State Response

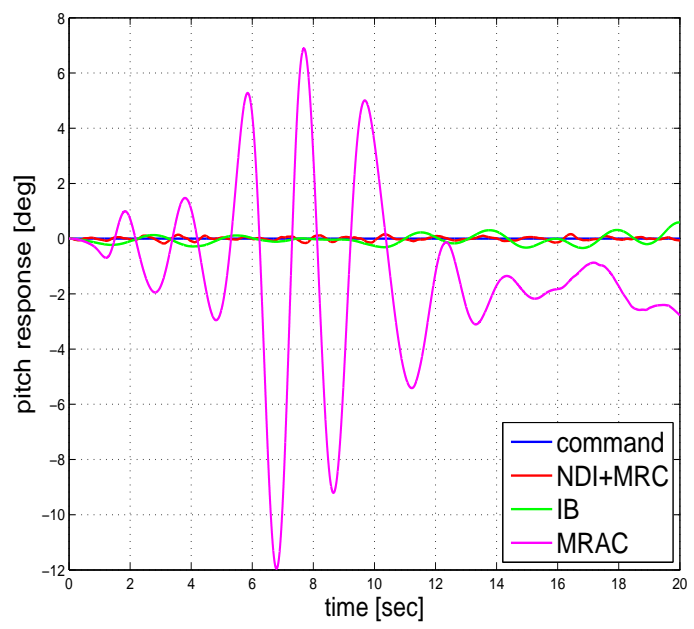

(c) Pitch State Response

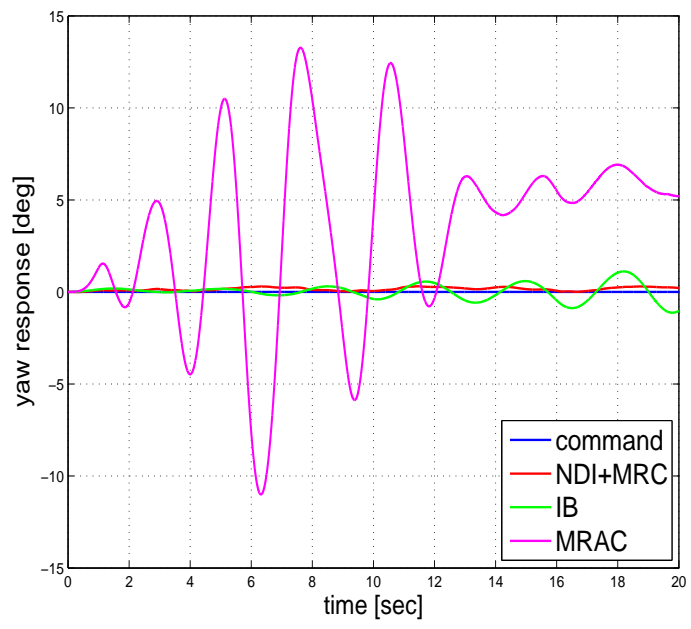

(e) Yaw State Response

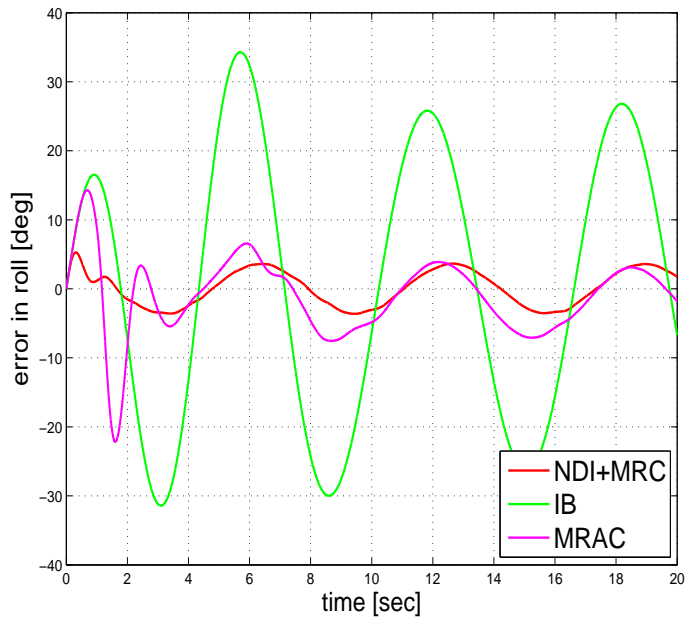

(b) Roll Error

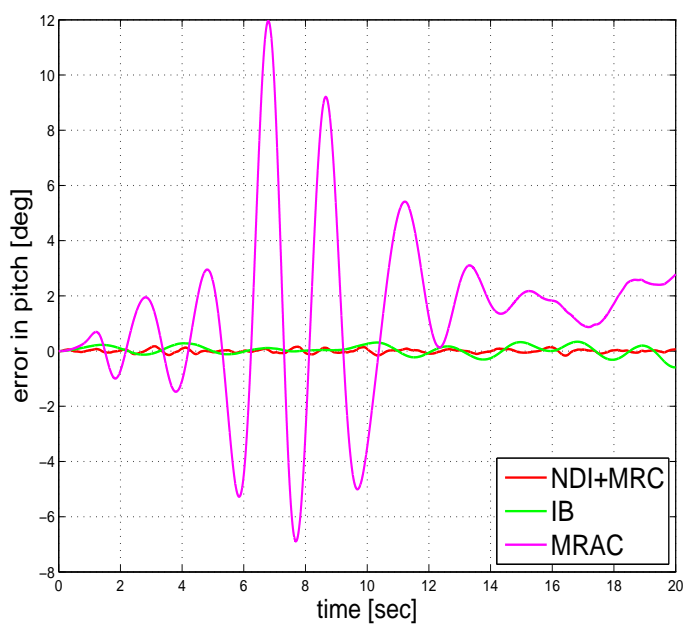

(d) Pitch Error

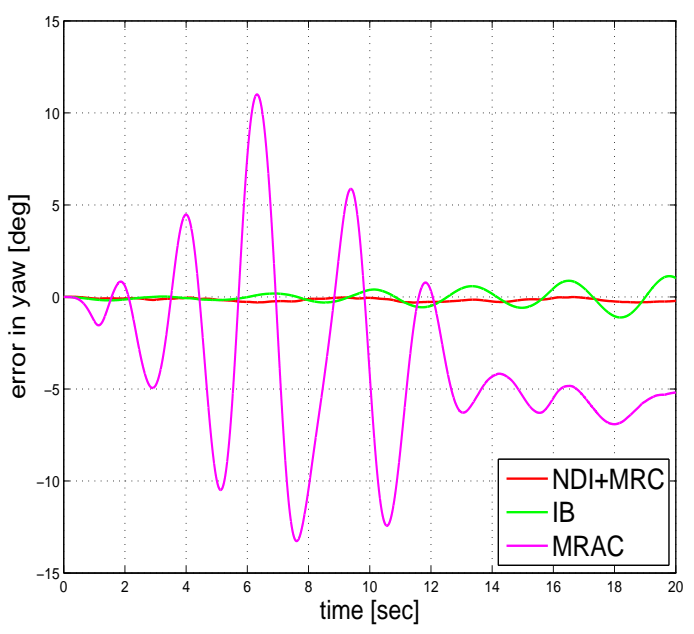

(f) Yaw Error

Figure B.3: Simulation of angular control for sinusoidal tracking with a different quadrotor model 


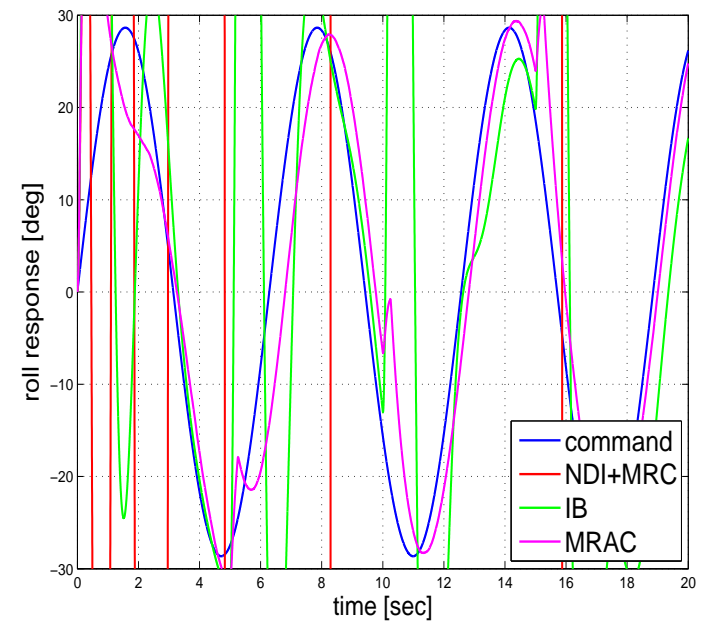

(a) Roll State Response

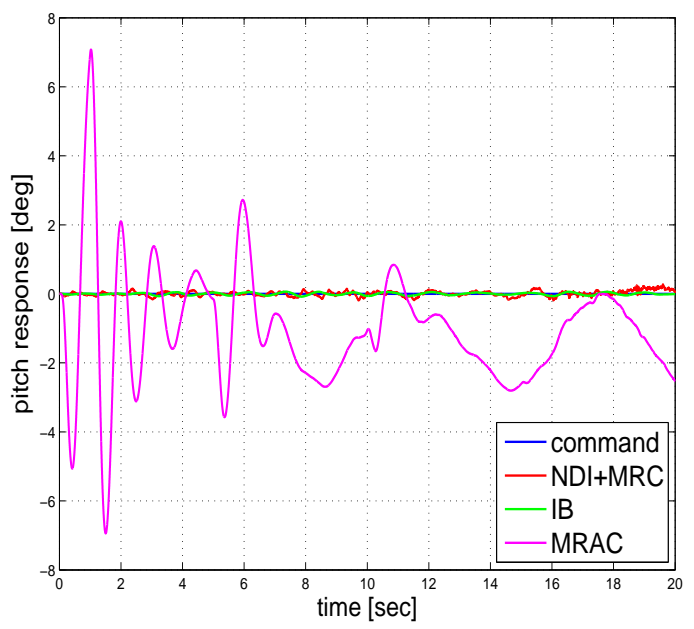

(c) Pitch State Response

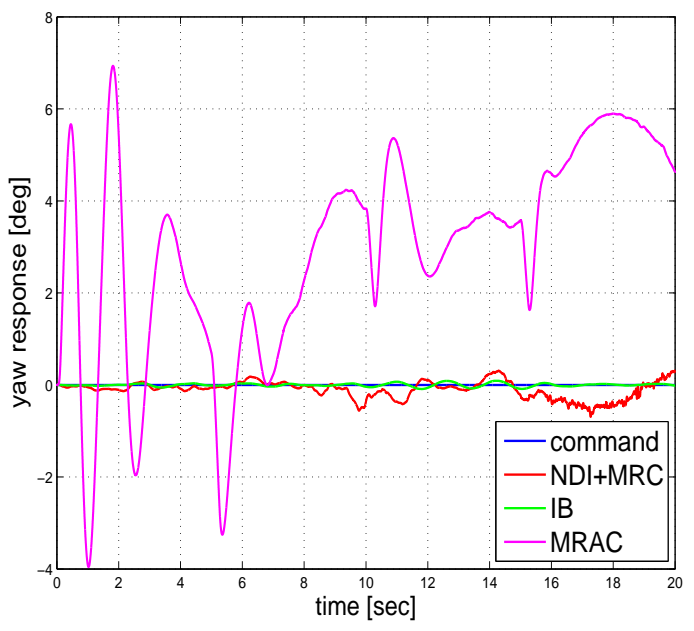

(e) Yaw State Response

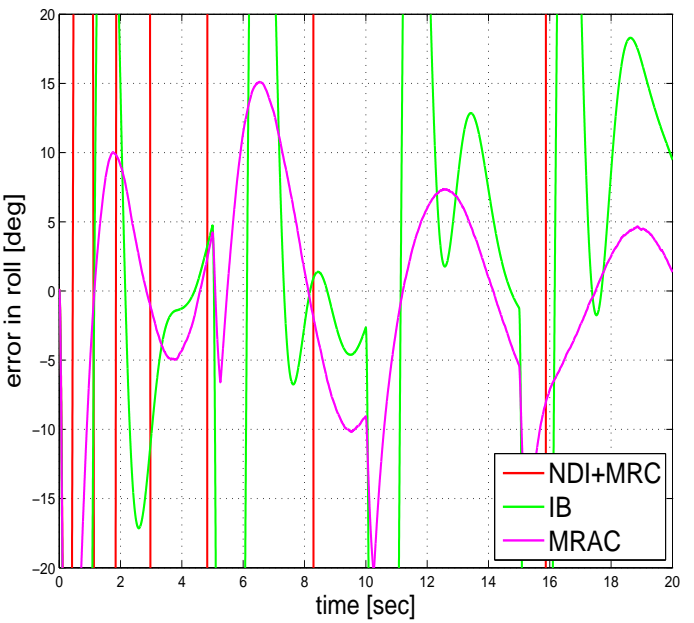

(b) Roll Error

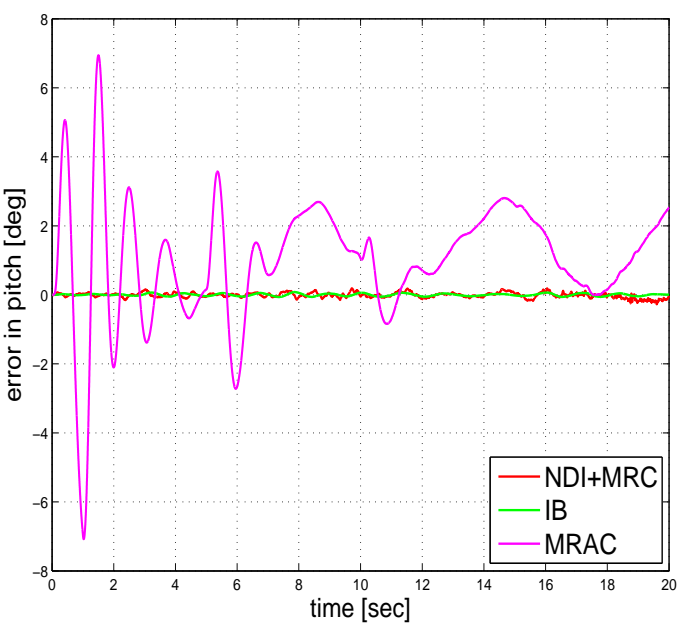

(d) Pitch Error

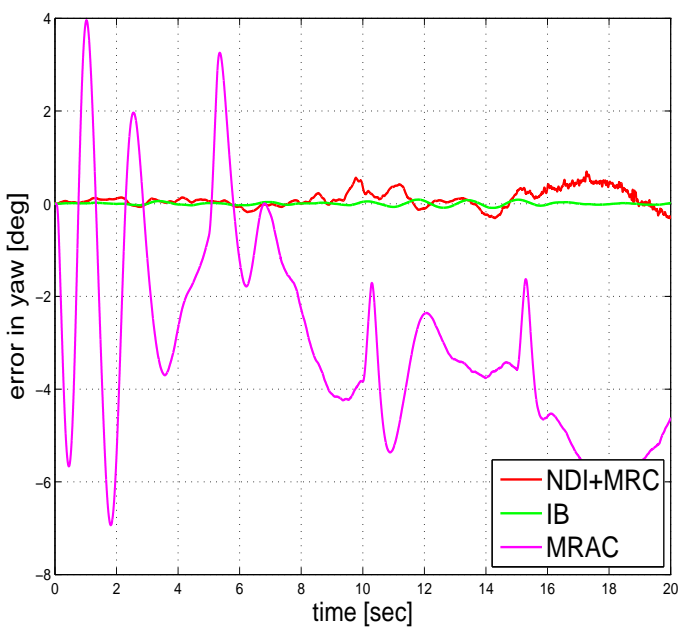

(f) Yaw Error

Figure B.4: Simulation of angular control for sinusoidal tracking in the presence of unknown disturbance 


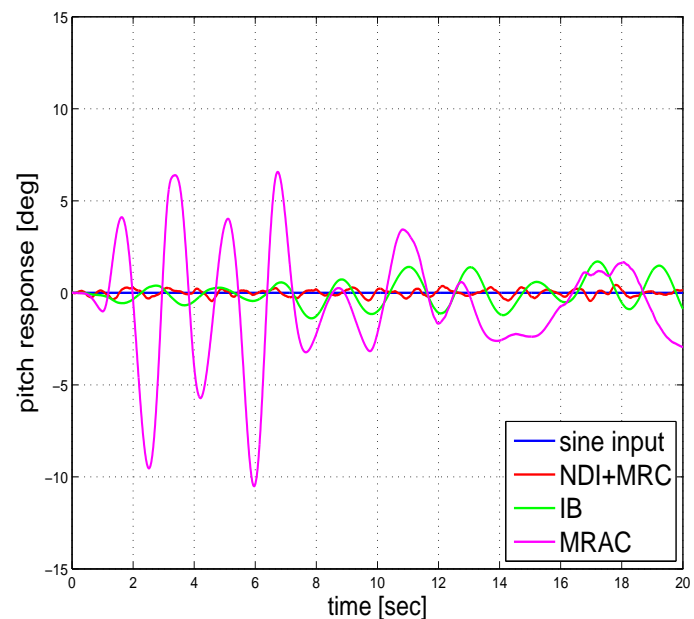

(a) Pitch Response for $\mathrm{A}=1$ and omega=1

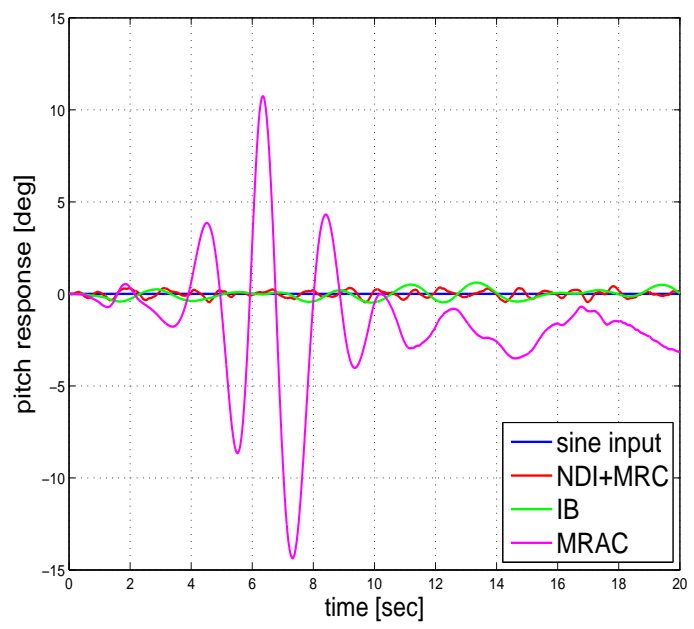

(c) Pitch Response for $\mathrm{A}=0.5$ and omega $=1$

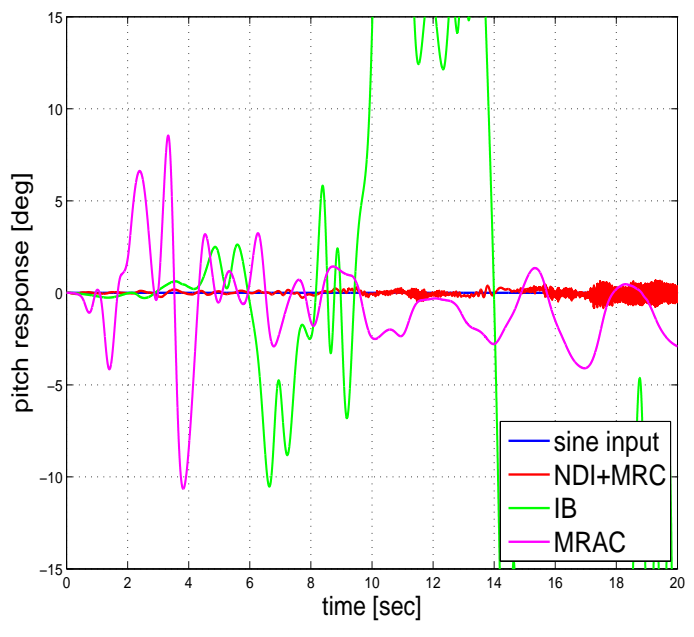

(b) Pitch Response for $\mathrm{A}=1$ and omega $=2$

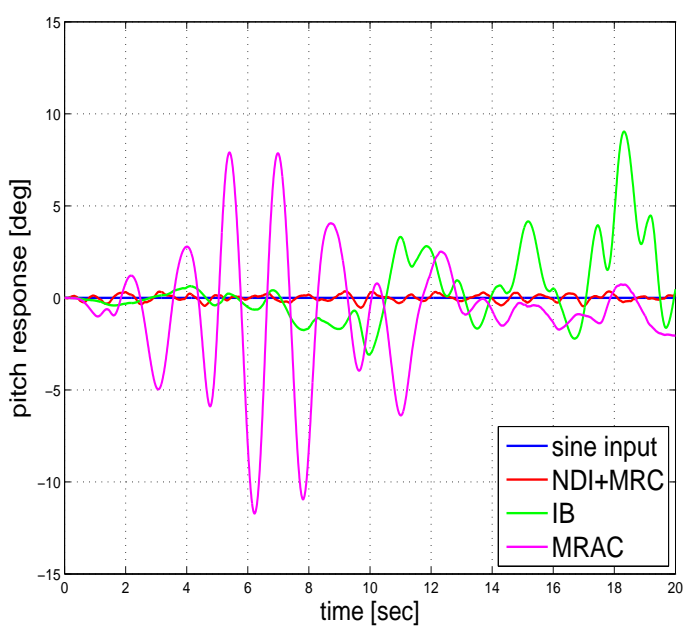

(d) Pitch Response for $\mathrm{A}=0.5$ and omega $=2$

Figure B.5: Simulation of sinusoidal command with various periods and amplitudes in the presence of unknown disturbance 


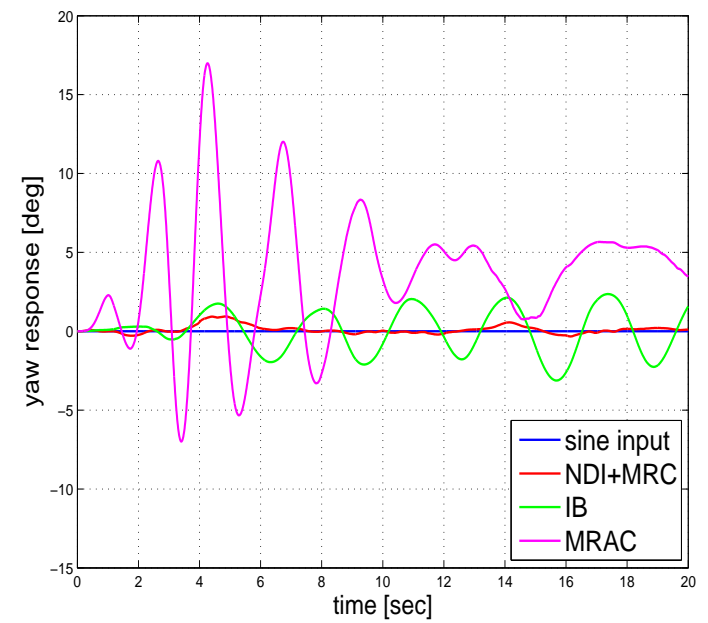

(a) Yaw Response for $\mathrm{A}=1$ and omega=1

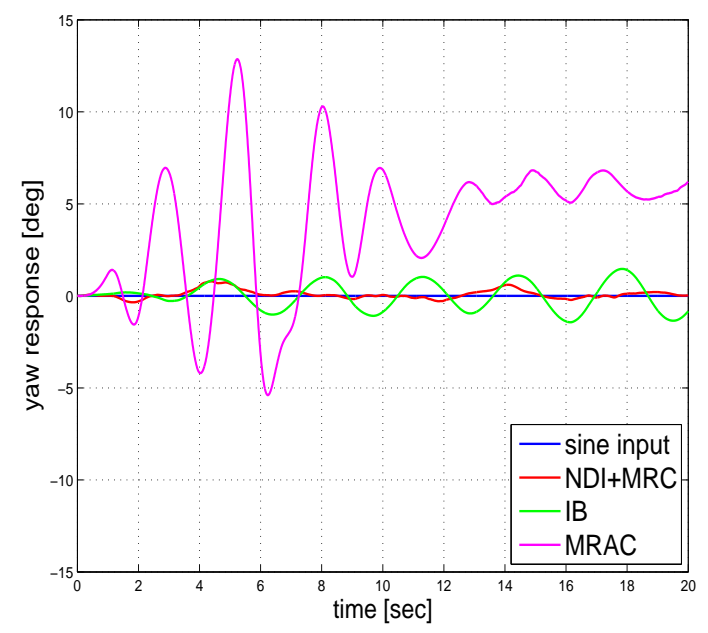

(c) Yaw Response for $\mathrm{A}=0.5$ and omega $=1$

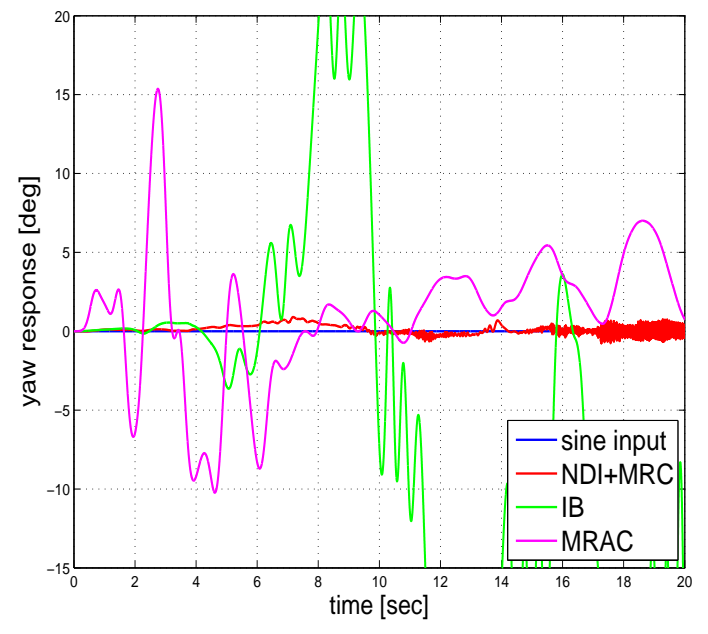

(b) Yaw Response for $\mathrm{A}=1$ and omega=2

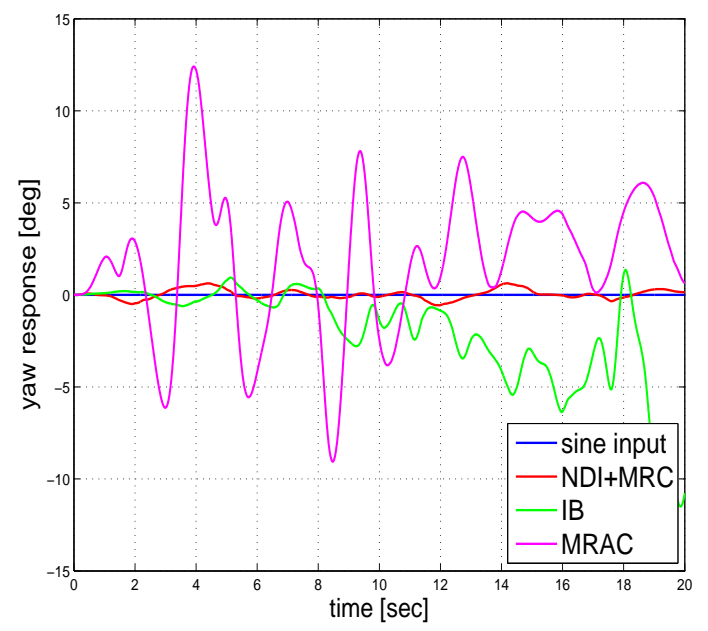

(d) Yaw Response for $\mathrm{A}=0.5$ and omega $=2$

Figure B.6: Simulation of sinusoidal command with various periods and amplitudes in the presence of unknown disturbance 


\section{APPENDIX C}

\section{FURTHER MODIFICATION RESULTS}

For the discussion of the figures in Appendix C, refer to Section 5.2 .

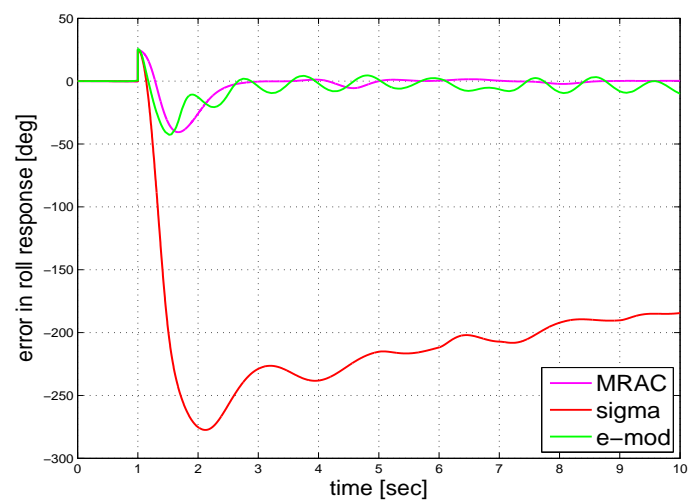

(a) Error in Roll Response

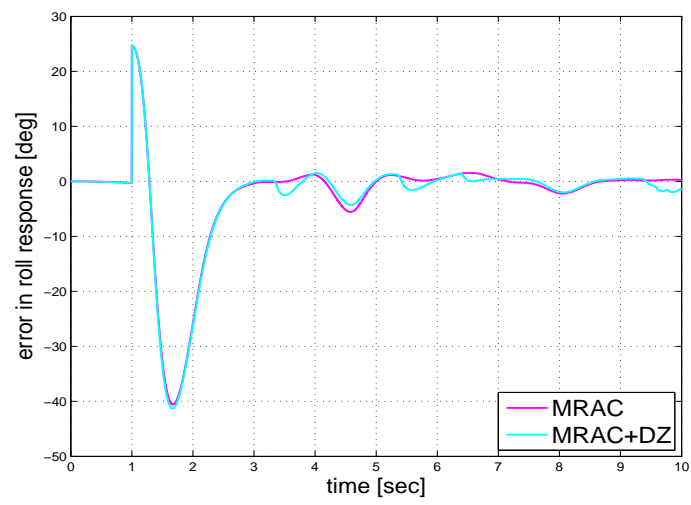

(c) Error in Roll Response

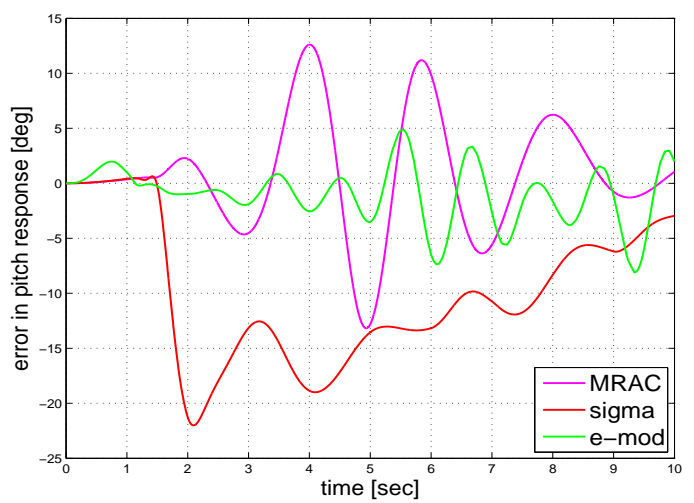

(b) Error in Pitch Response

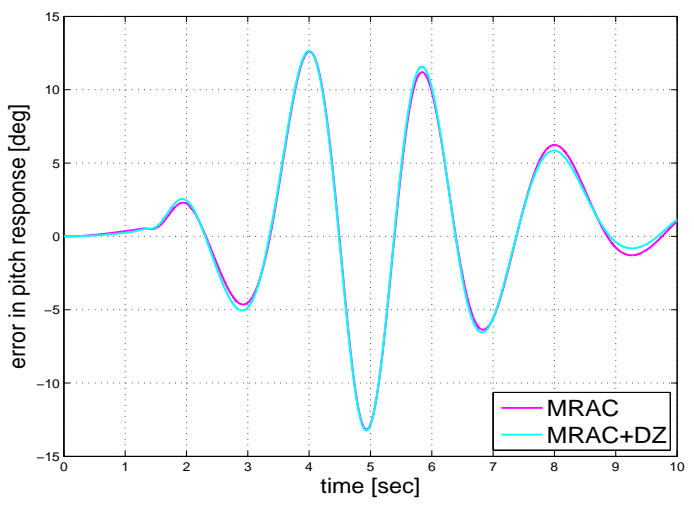

(d) Error in Pitch Response

Figure C.1: State Error Responses of Various MRAC Robustness Modifications for Step Roll-Input Trajectory Tracking in the presence of noise, parameter uncertainty and unknown disturbance 


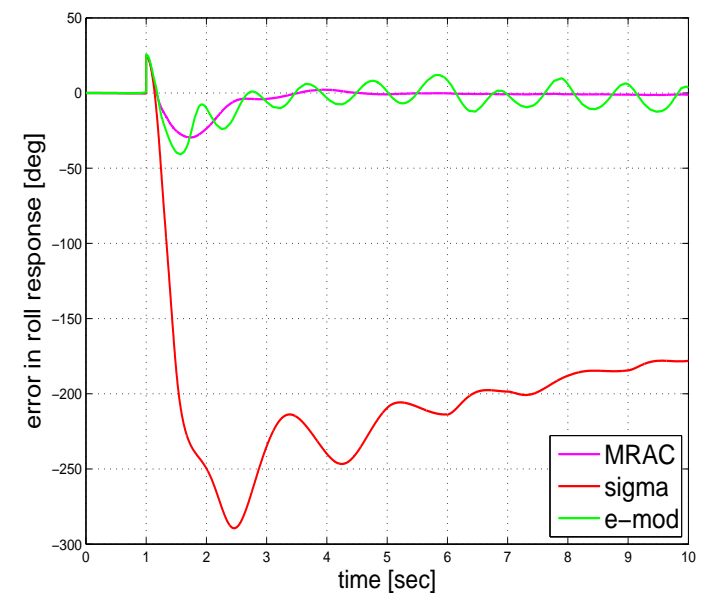

(a) Error in Roll Response

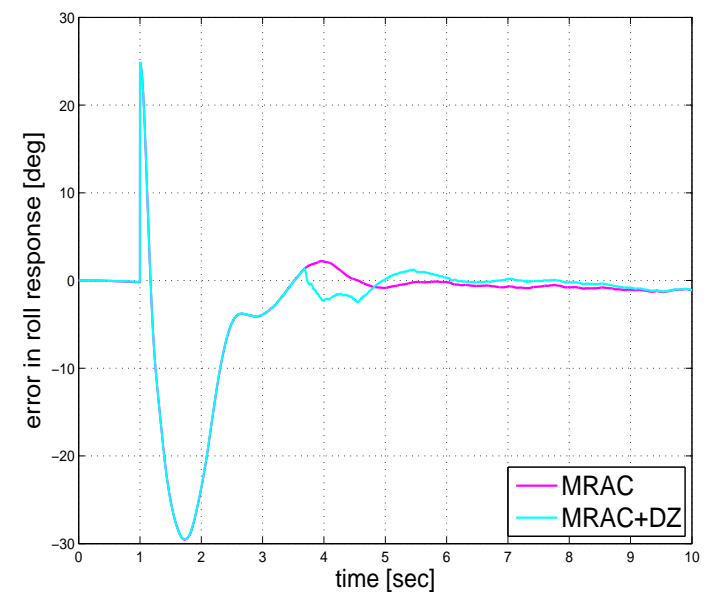

(c) Error in Roll Response

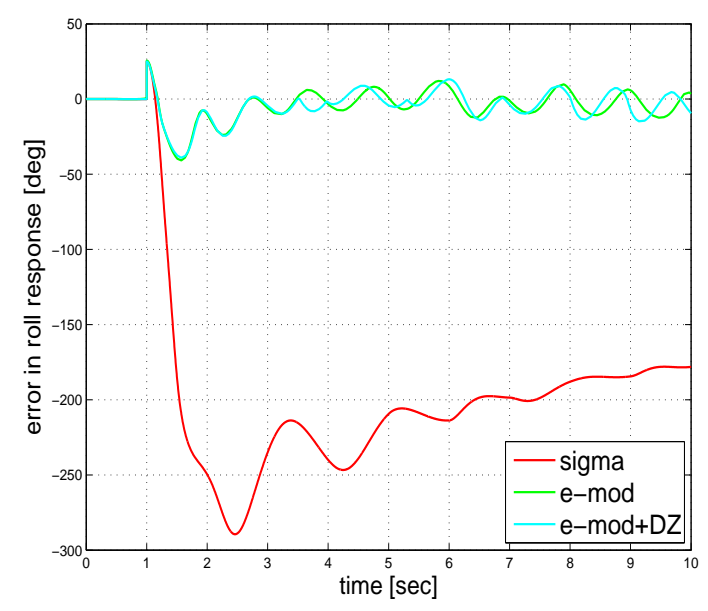

(e) Error in Roll Response

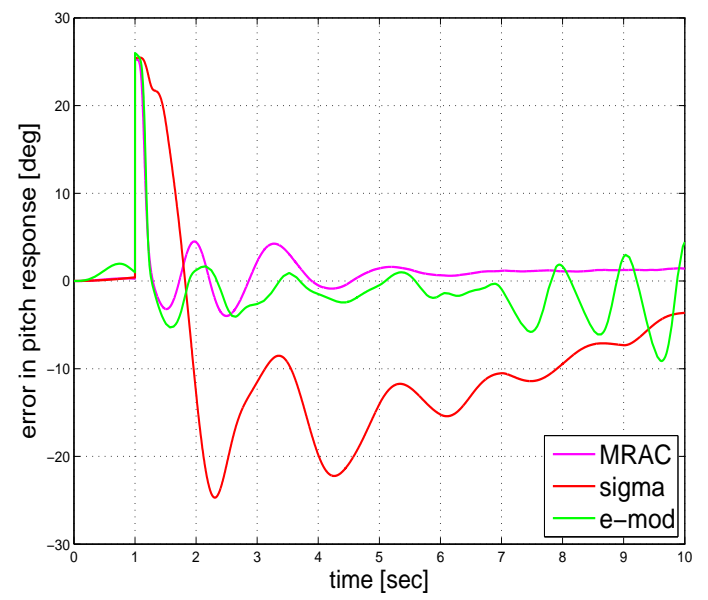

(b) Error in Pitch Response

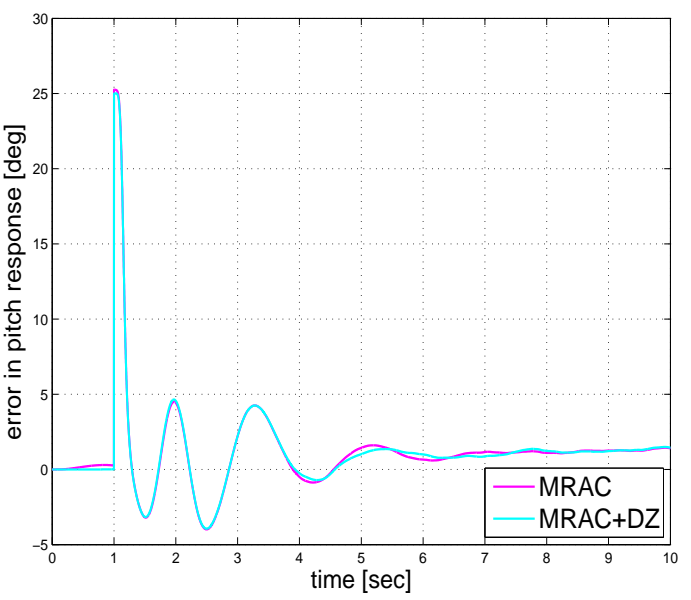

(d) Error in Pitch Response

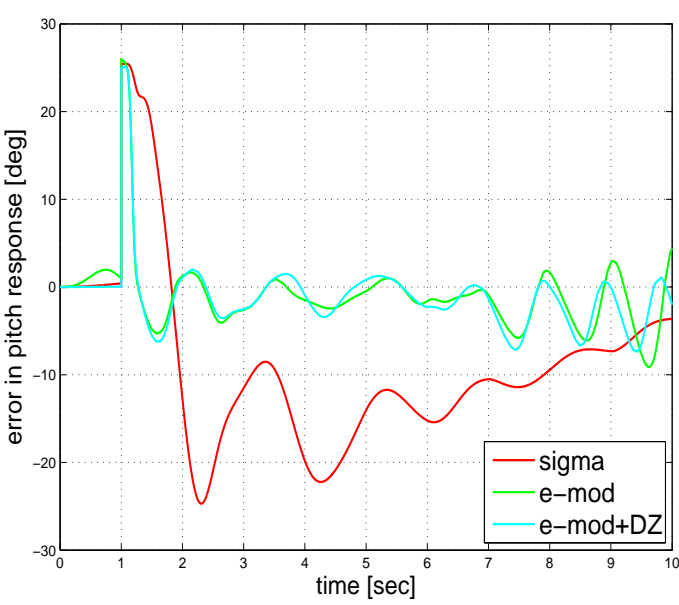

(f) Error in Pitch Response

Figure C.2: State Error Responses of Various MRAC Robustness Modifications for Step Roll and Pitch-Input Trajectory Tracking in the presence of noise and unknown disturbance 


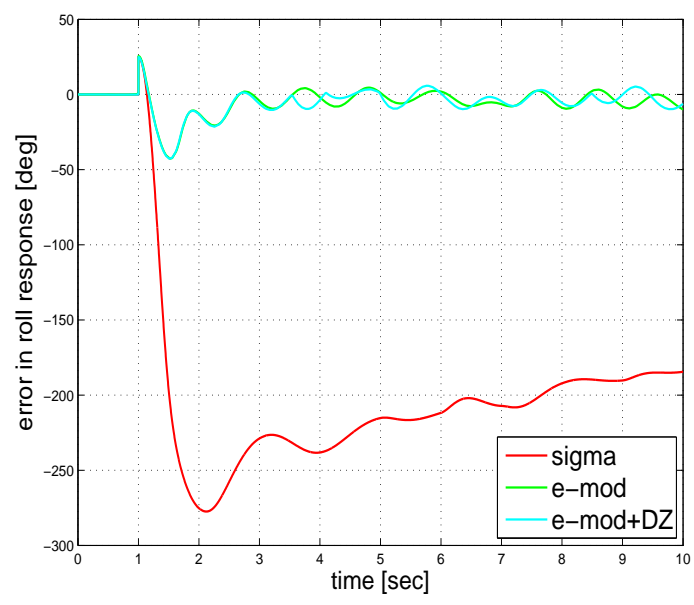

(a) Error in Roll Response

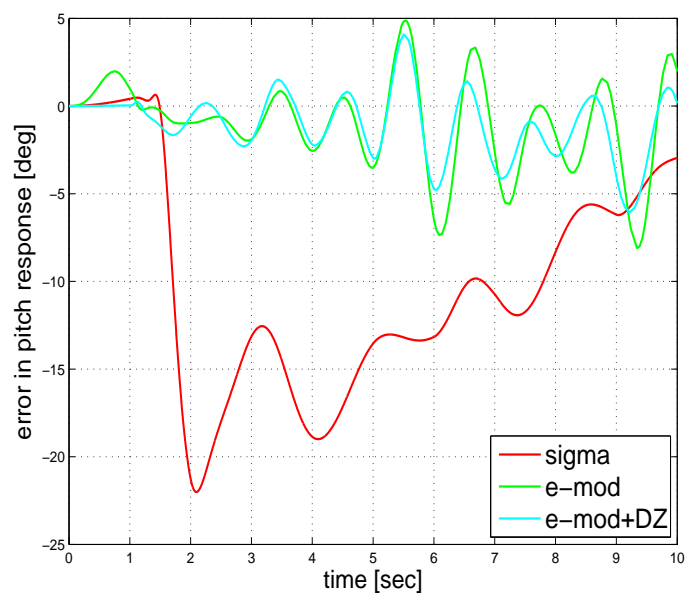

(b) Error in Pitch Response

Figure D.1: State Error Responses of Various MRAC Robustness Modifications for Step Roll-Input Trajectory Tracking in the presence of noise, parameter uncertainty and unknown disturbance 